QA: QA

ANL-MGR-MD-000003 REV 04

May 2005

\title{
Disruptive Event Biosphere Dose Conversion Factor Analysis
}

Prepared for:

U.S. Department of Energy

Office of Civilian Radioactive Waste Management

Office of Repository Development

1551 Hillshire Drive

Las Vegas, Nevada 89134-6321

Prepared by:

Bechtel SAIC Company, LLC

1180 Town Center Drive

Las Vegas, Nevada 89144

Under Contract Number

DE-AC28-01RW12101 


\section{DISCLAIMER}

This report was prepared as an account of work sponsored by an agency of the United States Government. Neither the United States Government nor any agency thereof, nor any of their employees, nor any of their contractors, subcontractors or their employees, makes any warranty, express or implied, or assumes any legal liability or responsibility for the accuracy, completeness, or any third party's use or the results of such use of any information, apparatus, product, or process disclosed, or represents that its use would not infringe privately owned rights. Reference herein to any specific commercial product, process, or service by trade name, trademark, manufacturer, or otherwise, does not necessarily constitute or imply its endorsement, recommendation, or favoring by the United States Government or any agency thereof or its contractors or subcontractors. The views and opinions of authors expressed herein do not necessarily state or reflect those of the United States Government or any agency thereof. 
QA: QA

Disruptive Event Biosphere Dose Conversion Factor Analysis ANL-MGR-MD-000003 REV 04

May 2005 


\section{OCRWM}

\section{Scientific Analysis Title}

Disruptive Event Biosphere Dose Conversion Factor Analysis

3. DI (including Revision Number)

ANL-MGR-MD-000003 REV 04

4. Total Appendices

3

3

\section{Originator}

7. Checker

8. QER

9. Responsible Manager/Lead

10. Responsible Manager

11. Remarks
5. Number of Pages in Each Appendix

$\mathrm{A}-6$ pages, $\mathrm{B}-6$ pages $+\mathrm{CD}, \mathrm{C}-4$ pages

\begin{tabular}{l} 
Printed Name \\
\hline Maryla A. Wasiolek \\
\hline D. Wesley Wu \\
Kenneth O. Gilkerson \\
\hline Maryla A. Wasiolek \\
Ming Zhu
\end{tabular}

\begin{tabular}{r|r|r} 
Signature $\cap$ & Date \\
\hline
\end{tabular}




\section{CONTENTS}

\section{Page}

ACRONYMS

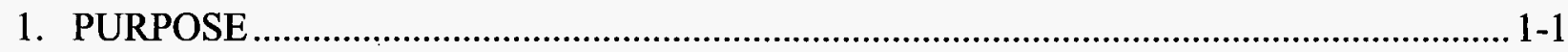

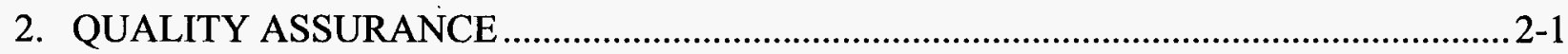

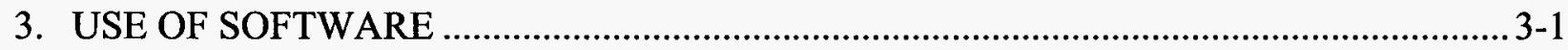

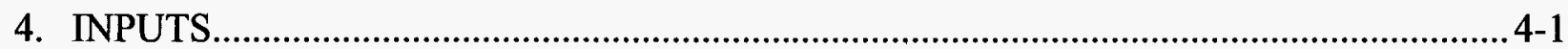

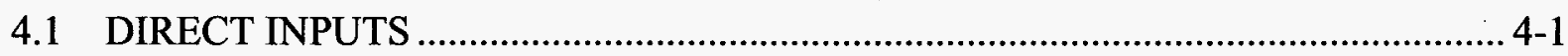

4.1.1 Direct Inputs Used for Calculation of Biosphere Dose Conversion Factors

4.1.2 Direct Inputs Used for Calculation of Inhalation Dose Factors for

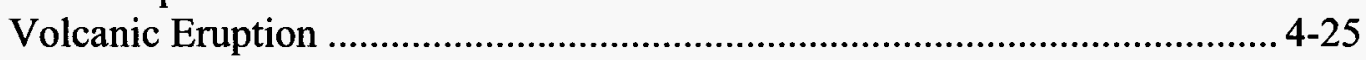

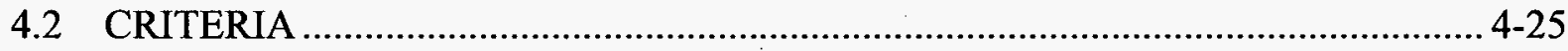

4.3 CODES, STANDARDS, AND REGULATIONS .................................................... 4-28

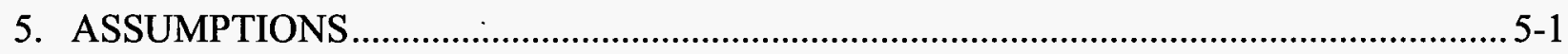

6. SCIENTIFIC ANALYSIS DISCUSSION............................................................................

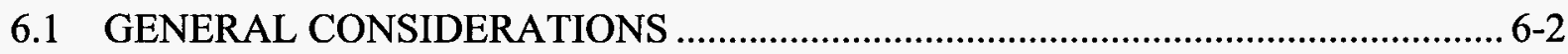

6.1.1 Radionuclides Included in the Analysis ............................................................ 6-2

6.1.2 Description of the Volcanic Ash Exposure Scenario ............................................ 6-2

6.1.3 Consideration of Climate Change ……………............................................ 6-5

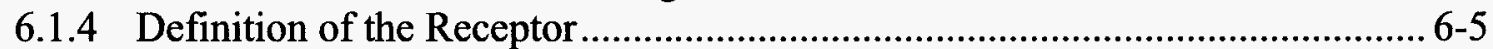

6.1.5 Biosphere Model ................................................................................. 6-6

6.2 DEVELOPMENT OF BIOSPHERE DOSE CONVERSION FACTORS FOR

THE VOLCANIC ASH EXPOSURE SCENARIO ……......................................... 6-8

6.2.1 Treatment of Uncertainty ............................................................................ 6-9

6.2.2 Format of Biosphere Dose Conversion Factors for Volcanic Ash Exposure Scenario and Their Use in the Total System Performance Assessment Model............................................................................................ 6-9

6.2.3 Results of the Calculations .................................................................. 6-12

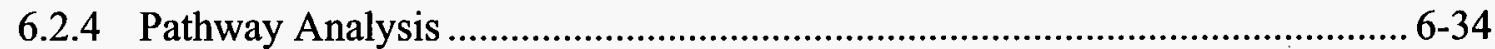

6.2.5 Climate Change ....................................................................................... 6-38

6.3 INHALATION DOSE FACTORS FOR THE ERUPTION PHASE ………............... 6-40

6.3.1 Mass Loading Levels During Volcanic Eruption.............................................. 6-41

6.3.2 Development of Inhalation Dose Factors........................................................ 6-43

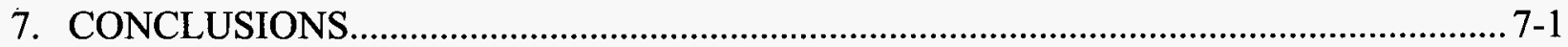

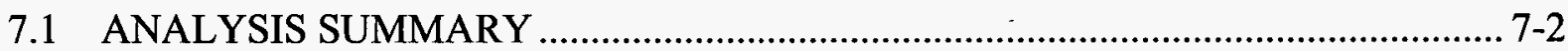

7.1.1 Incorporation of Uncertainty ...................................................................... 7-2

7:1.2 Format of the Biosphere Dose Conversion Factor Input to TSPA..................... 7-3 


\section{CONTENTS (CONTINUED)}

Page

7.1.3 Use of Biosphere Dose Conversion Factors for Volcanic Ash Exposure Scenario

7.1.4 Use of Inhalation Dose Factors .................................................................. 7-5

7.1.5 Correlations, Pathway Analysis, and Climate Change..................................... 7-6

7.2 HOW ACCEPTANCE CRITERIA ARE ADDRESSED …………........................... 7-6

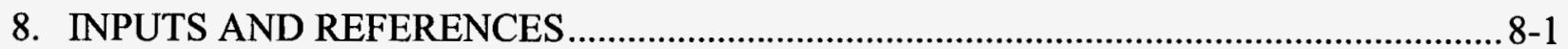

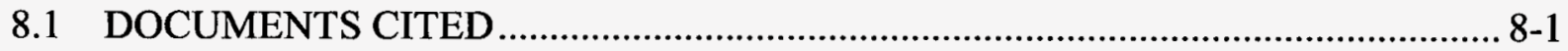

8.2 CODES, STANDARDS, REGULATIONS, AND PROCEDURES ……….............. 8-5

8.3 SOURCE DATA, LISTED BY DATA TRACKING NUMBER ………................. 8-5

8.4 SOFTWARE CODES ................................................................................................. 8-6

8.5 OUTPUT DATA, LISTED BY DATA TRACKING NUMBER ….......................... 8-6

APPENDIX A - LIST AND DESCRIPTION OF GOLDSIM FILES GENERATED IN THIS ANALYSIS

APPENDIX B-- DESCRIPTION OF EXCEL FILES GENERATED IN THIS ANALYSIS

APPENDIX C - VERIFICATION OF ERMYN MODEL FILES FOR GOLDSIM VERSIONS 8.01 SERVICE PACK 4 AND 8.02.300 


\section{FIGURES}

1-1. Biosphere Model Documentation.

6.1-1. Conceptual Representation of the Biosphere Model for the Volcanic Ash Exposure Scenario .

6.1-2. Relationship Between the Biosphere Submodels for the Volcanic Ash Exposure Scenario .

6.2-1. Comparison of ICRP 30- and ICRP 72-Based BDCF Components for External Exposure, Ingestion, and Inhalation of Radon Decay Products for the PresentDay Climate.

6.2-2. Comparison of ICRP 30- and ICRP 72-Based BDCF Components for Inhalation of Particulate Matter for the Present-Day Climate

6.2-3. Mean BDCFs as a Function of Time

6.2-4. Mean BDCFs as a Function of Ash Thickness 6-38

A-1. GoldSim Files for Calculating Volcanic Scenario BDCFs for the Present-Day Climate Generated Using ICRP 72-Based Dosimetric Inputs

A-2. GoldSim Files for Calculating Volcanic Scenario BDCFs for the Upper Bound of the Glacial Transition Climate Generated Using ICRP 72-Based Dosimetric Inputs

A-3. GoldSim Files for Calculating Volcanic Scenario BDCFs for the Present-Day Climate Using GoldSim 8.01 Service Pack 4 and ICRP 30-Based Dosimetric Inputs

A-4. GoldSim Files for Calculating Volcanic Scenario BDCFs for the Upper Bound of the Glacial Transition Climate Using GoldSim 8.01 Service Pack 4 and ICRP 30Based Dosimetric Inputs

B-1. List of Excel Files Supporting This Analysis B-1

C-1. Model Verification Files Obtained Using GoldSim Versions 7.50.100, 8.01 Service Pack 4, and 8.02.300. 


\section{INTENTIONALLY LEFT BLANK}




\section{TABLES}

1-1. Biosphere-Related Features, Events, and Processes Implicitly Included in TSPA through the Use of BDCFs for the Volcanic Ash Exposure Scenario

1-2. Features, Events, and Processes Related to Inhalation Dose Factors for the Eruption Phase.....

4.1-1. Summary of Input Parameter Values and Their Uncertainty Distributions ...................4-2

4.1-2. Primary Radionuclides and Their Decay Products Included in the Biosphere Model for the Volcanic Ash Exposure Scenario.....

4.1-3. Dose Coefficients for Inhalation and Ingestion of Radionuclides of Interest Based on ICRP 30 Dosimetric Methods.

4.1.4. Dose Coefficients for Inhalation and Ingestion of Radionuclides of Interest Based on ICRP 72 Dosimetric Methods.

4.1-5. Dose Coefficients for Exposure to Contaminated Soil Surface for Radionuclides of Interest.

4.1-6. Population Proportion Values Used for Calculating Inhalation Dose Factors ..............4-25

4.2-1. Requirements Applicable to this Analysis

6.1-1. RMEI Exposure Pathways for the Volcanic Ash Exposure Scenario

6.2-1. BDCF Component for External Exposure, Ingestion, and Inhalation of Radon Decay Products for the ICRP 30-Based Dosimetry and Present-Day Climate, $\mathrm{rem} / \mathrm{yr}$ per $\mathrm{pCi} / \mathrm{m}^{2}$

6.2-2. BDCF Component for Short-Term Inhalation Exposure for the ICRP 30-Based Dosimetry and Present-Day Climate, $\mathrm{rem} / \mathrm{yr}$ per $\mathrm{pCi} / \mathrm{m}^{2}$

6.2-3. BDCF Component for Long-Term Inhalation Exposure for the ICRP 30-Based Dosimetry and Present-Day Climate, rem $/ \mathrm{yr}$ per $\mathrm{pCi} / \mathrm{m}^{2}$

6.2-4. BDCF Component for External Exposure, Ingestion, and Inhalation of Radon Decay Products for the ICRP 72-Based Dosimetry and Present-Day Climate, $\mathrm{rem} / \mathrm{yr}$ per $\mathrm{pCi} / \mathrm{m}^{2}$

6.2-5. BDCF Component for Short-Term Inhalation Exposure for the ICRP 72-Based Dosimetry and Present-Day Climate, $\mathrm{rem} / \mathrm{yr}$ per $\mathrm{pCi} / \mathrm{m}^{2}$

6.2-6. BDCF Component for Long-Term Inhalation Exposure for the ICRP 72-Based Dosimetry and Present-Day Climate, $\mathrm{rem} / \mathrm{yr}$ per $\mathrm{pCi} / \mathrm{m}^{2}$

6.2-7. Correlation Coefficients for the Volcanic Ash Scenario ICRP 30-Based BDCF Components

6.2-8. Correlation Coefficients for the Volcanic Ash Scenario ICRP 72-Based BDCF Components 6-28

6.2-9. Calculated Values of Correlation Coefficient and Variable $t$ 6-29

6.2-10. Correlation Coefficients for the External Exposure-Ingestion-Radon ICRP 30Based BDCF Components for Individual Radionuclides

6.2-11. Correlation Coefficients for the External Exposure-Ingestion-Radon ICRP 72-

Based BDCF Components for Individual Radionuclides

6.2-12. Exposure Pathway Contributions (Percent) for the Mean Volcanic Ash Exposure Scenario ICRP 30-Based BDCFs for the Present-Day Climate 6-35

6.2-13. Exposure Pathway Contributions (Percent) for the Mean Volcanic Ash Exposure

Scenario ICRP 72-Based BDCFs for the Present-Day Climate 
6.2-14. Ratio of Mean Ingestion Pathway ICRP 30-Based BDCFs for the Volcanic Ash Exposure Scenario and the Future and Present-Day Climates

6.2-15. Ratio of Mean Ingestion Pathway ICRP 72-Based BDCFs for the Volcanic Ash Exposure Scenario and the Future and Present-Day Climates .....................................6-40

6.3-1. Effective Dose Coefficients for Inhalation Consistent with ICRP 30 Methods ............ 6-47

6.3-2. Effective Dose Coefficients for Inhalation Consistent with ICRP 72 Methods ............6-49

6.3-3. Supplementary Calculations Supporting Development of Inhalation Dose Factors ....6-51

6.3-4. ICRP 30-Based Inhalation Dose Factors for Eruptive Phase of the Volcanic Scenario

6.3-5. ICRP 72-Based Inhalation Dose Factors for Eruptive Phase of the Volcanic Scenario $6-53$

7-1. Output Data Tracking Numbers - ICRP 30 Dosimetric Methods

7-2. Output Data Tracking Numbers - ICRP 72 Dosimetric Methods

7.1-1. Primary Radionuclides and Their Decay Products Included in the BDCFs for the Volcanic Ash Exposure Scenario

C-1. Results of Verification Runs Using GoldSim Versions 7.50.100, 8.01 Service Pack 4, and 8.02.300 


\section{ACRONYMS}

AC acceptance criterion

BDCF biosphere dose conversion factor

DOE U.S. Department of Energy

ERMYN Environmental Radiation Model for Yucca Mountain, Nevada

FEP feature, event, and process

FGR Federal Guidance Report

ICRP International Commission on Radiological Protection

IRT International Review Team

LA license application

NRC U.S. Nuclear Regulatory Commission

QA quality assurance

RMEI reasonably maximally exposed individual

TSP total suspended particulate

TSPA total system performance assessment

TWP technical work plan 


\section{INTENTIONALLY LEFT BLANK}




\section{PURPOSE}

This analysis report is one of the technical reports containing documentation of the Environmental Radiation Model for Yucca Mountain, Nevada (ERMYN), a biosphere model supporting the total system performance assessment (TSPA) for the license application (LA) for the Yucca Mountain repository. This analysis report describes the development of biosphere dose conversion factors (BDCFs) for the volcanic ash exposure scenario, and the development of dose factors for calculating inhalation dose during volcanic eruption.

A graphical representation of the documentation hierarchy for the ERMYN is presented in Figure 1-1. This figure shows the interrelationships among the products (i.e., analysis and model reports) developed for biosphere modeling and provides an understanding of how this analysis report contributes to biosphere modeling. This report is one of two reports that develop biosphere BDCFs, which are input parameters for the TSPA model. The Biosphere Model Report (BSC 2004 [DIRS 169460]) describes in detail the ERMYN conceptual model and mathematical model. The input parameter reports, shown to the right of the Biosphere Model Report in Figure 1-1, contain detailed descriptions of the model input parameters, their development and the relationship between the parameters and specific features, events and processes (FEPs). This report describes biosphere model calculations and their output, the BDCFs, for the volcanic ash exposure scenario. This analysis receives direct input from the outputs of the Biosphere Model Report (BSC 2004 [DIRS 169460]) and from the five analyses that develop parameter values for the biosphere model (BSC 2005 [DIRS 172827]; BSC 2004 [DIRS 169672]; BSC 2004 [DIRS 169673]; BSC 2004 [DIRS 169458]; and BSC 2004 [DIRS 169459]). The results of this report are further analyzed in the Biosphere Dose Conversion Factor Importance and Sensitivity Analysis (Figure 1-1).

The objective of this analysis was to develop the BDCFs for the volcanic ash exposure scenario and the dose factors for calculating inhalation doses during volcanic eruption (eruption phase of the volcanic event). For the volcanic ash exposure scenario, the mode of radionuclide release into the biosphere is a volcanic eruption through the repository with the resulting entrainment of contaminated waste in the tephra and the subsequent atmospheric transport and dispersion of contaminated material in the biosphere. The biosphere process model for this scenario uses the surface deposition of contaminated ash as the source of radionuclides in the biosphere. The initial atmospheric transport and dispersion of the ash as well as its subsequent redistribution by fluvial and aeolian processes are not addressed within the biosphere model. These processes influence the value of the source term that is calculated elsewhere and then combined with the BDCFs in the TSPA model to calculate expected dose to the receptor.

This analysis is a revision of the Disruptive Event Biosphere Dose Conversion Factor Analysis (BSC 2004 [DIRS 167287]). The scope of the revision was to calculate BDCFs and the dose factors for evaluating inhalation doses during volcanic eruption using updated dosimetric input parameters from, or consistent with, the International Commission on Radiological Protection (ICRP) Publication 72 (ICRP 72) (ICRP 1996 [DIRS 152446]). The quantities developed previously (BSC 2004 [DIRS 167287]), that use dosimetric inputs consistent with ICRP Publication 30 (ICRP 30) (ICRP 1979 [DIRS 110386]; ICRP 1980 [DIRS 110351]; ICRP 1981 [DIRS 110352]) were retained for comparison purposes. 


\section{LA FEP List \\ (Biosphere)}

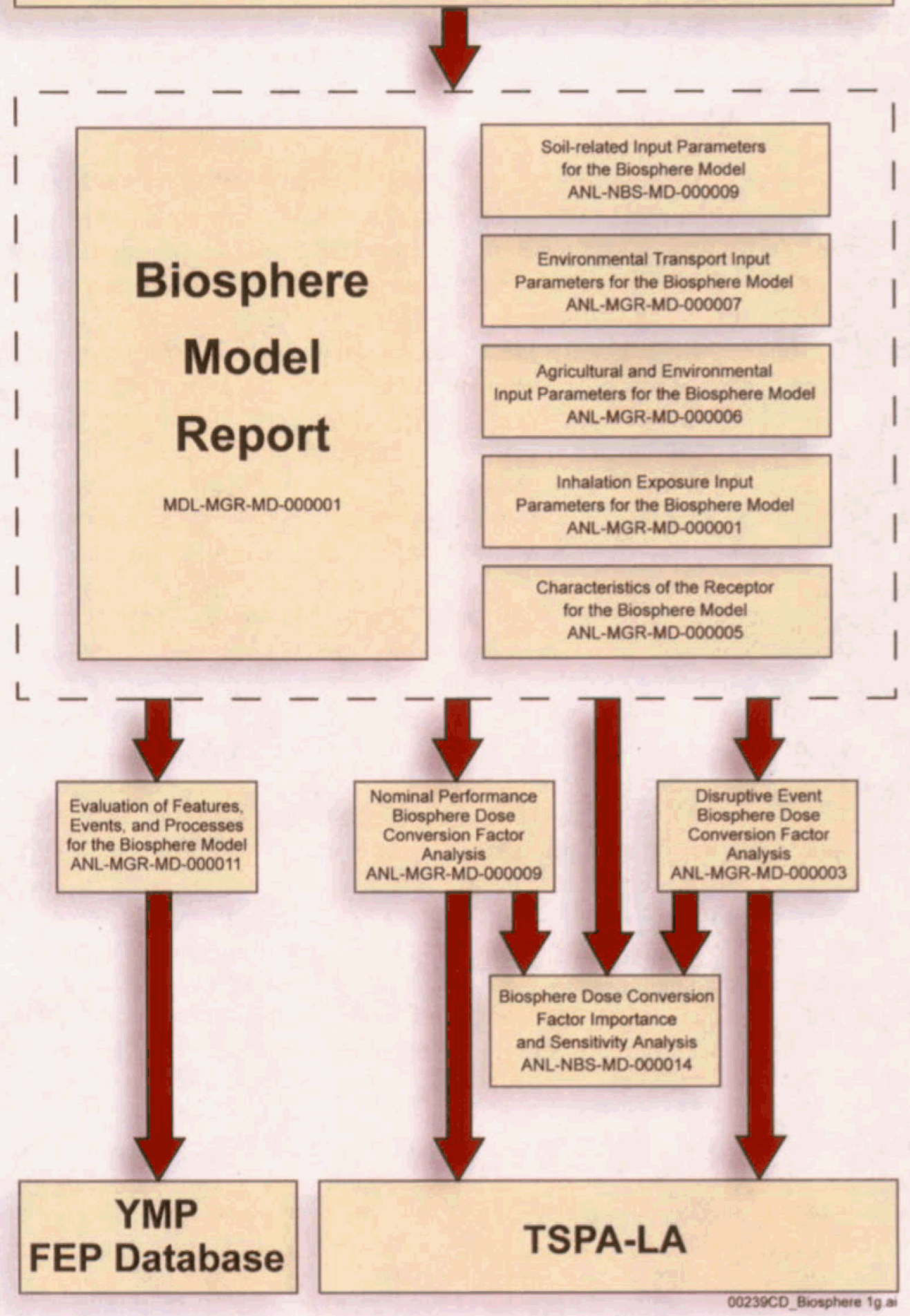

Figure 1-1. Biosphere Model Documentation 
Dosimetric inputs consistent with ICRP Publication 30 are based on the concepts recommended in ICRP Publication 26 (ICRP 1977 [DIRS 101075]) and the dosimetric methods for intakes of radionuclides by workers outlined in ICRP Publication 30 (ICRP 1979 [DIRS 110386]; ICRP 1980 [DIRS 110351]; ICRP 1981 [DIRS 110352]). This approach is consistent with the individual protection standard defined in terms of total effective dose equivalent.

Dosimetric inputs consistent with ICRP Publication 72 are based on the concepts recommended in ICRP Publication 60 (ICRP 1991 [DIRS 101836]) and the updated biokinetic and dosimetric models for intakes of radionuclides by members of the public. ICRP Publication 60 introduced a new dosimetric quantity, the effective dose, which uses an expanded list of tissues and organs and revised values of tissue and organ weighting factors. In addition, biokinetic and dosimetric models were updated to include new information that became available since ICRP Publication 30. A new set of age-dependent dose coefficients for intakes of radionuclides by members of the public was developed (ICRP Publication 30 concerned doses to workers) and documented in a series of reports compiled in ICRP Publication 72 (ICRP 1996 [DIRS 152446]). ICRP Publication 72 is presently the most current compilation of dose coefficients for internal intakes.

The biosphere model considers FEPs applicable to the Yucca Mountain biosphere (DTN: MO0501SEPFEPLA.001 [DIRS 172601]). Table 1-1 lists FEPs that are included in the biosphere model for the volcanic ash exposure scenario (BSC 2004 [DIRS 169460], Tables 6.2-1 and 6.3-6). Relationships among the biosphere-related FEPs, the biosphere conceptual model, and the exposure scenarios are examined in the Biosphere Model Report (BSC 2004 [DIRS 169460], Section 6.3); the disposition of the included FEPs within the mathematical model, and their explicit relationship to the model equations and input parameters is presented in the Biosphere Model Report (BSC 2004 [DIRS 169460], Table 6.7-1). The Biosphere Model Report (BSC 2004 [DIRS 169460]) uses a previous LA FEPs List (DTN: MO0407SEPFEPLA.000 [DIRS 170760]). However, the names, descriptions, and dispositions of the biosphere-related FEPs are the same in both sets.

The BDCFs developed as output of this report, and used as direct inputs to the TSPA, are the results of the biosphere model and use inputs from the supporting parameter reports. The BDCFs, therefore, implicitly include the FEPs that are related to the model and to the model inputs taken from the supporting parameter reports. Consequently, explicit mapping of individual FEPs listed in Table 1-1 to specific sections of this report and BDCFs is not possible. All FEPs considered in the biosphere model and its input parameters are collectively included in the BDCFs.

In addition to producing the BDCFs for the volcanic ash exposure scenario, this analysis develops the values of inhalation dose factors for calculation of inhalation doses during a volcanic eruption. Inhalation dose factors are multipliers, similar to BDCFs, and are used in the TSPA to evaluate inhalation dose accrued during a volcanic eruption. The BDCFs do not include contributions from inhalation of the falling contaminated ash. They are developed for the conditions following ash deposition and are calculated using the ERMYN model in terms of annual doses. The ERMYN is not used to calculate inhalation dose factors. The FEPs that are related to inhalation dose factors are listed in Table 1-2. The disposition of these FEPs in TSPA is through the dose factors that are used in the TSPA calculations. 
The analysis was performed in accordance with LP-SIII.9Q-BSC, Scientific Analyses, and the Technical Work Plan for Biosphere Modeling and Expert Support (TWP) (BSC 2005 [DIRS 172782]).

Table 1-1. Biosphere-Related Features, Events, and Processes Implicitly Included in TSPA through the Use of BDCFs for the Volcanic Ash Exposure Scenario

\begin{tabular}{|l|c|}
\hline \multicolumn{1}{|c|}{ FEP Name } & LA FEP Number \\
\hline Ashfall & $1.2 .04 .07 .0 \mathrm{~A}$ \\
\hline Climate change & $1.3 .01 .00 .0 \mathrm{~A}$ \\
\hline Soil type & $2.3 .02 .01 .0 \mathrm{~A}$ \\
\hline Radionuclide accumulation in soils & $2.3 .02 .02 .0 \mathrm{~A}$ \\
\hline Soil and sediment transport in the biosphere & $2.3 .02 .03 .0 \mathrm{~A}$ \\
\hline Precipitation & $2.3 .11 .01 .0 \mathrm{~A}$ \\
\hline Biosphere characteristics & $2.3 .13 .01 .0 \mathrm{~A}$ \\
\hline Radionuclide alteration during biosphere transport & $2.3 .13 .02 .0 \mathrm{~A}$ \\
\hline Human characteristics (physiology, metabolism) & $2.4 .01 .00 .0 \mathrm{~A}$ \\
\hline Human lifestyle & $2.4 .04 .01 .0 \mathrm{~A}$ \\
\hline Dwellings & $2.4 .07 .00 .0 \mathrm{~A}$ \\
\hline Wild and natural land and water use & $2.4 .08 .00 .0 \mathrm{~A}$ \\
\hline Agricultural land use and irrigation & $2.4 .09 .01 .0 \mathrm{~B}$ \\
\hline Animal farms and fisheries & $2.4 .09 .02 .0 \mathrm{~A}$ \\
\hline Urban and industrial land and water use & $2.4 .10 .00 .0 \mathrm{~A}$ \\
\hline Radioactive decay and ingrowth & $3.1 .01 .01 .0 \mathrm{~A}$ \\
\hline Atmospheric transport of contaminants & $3.2 .10 .00 .0 \mathrm{~A}$ \\
\hline Contaminated drinking water, foodstuffs and drugs & $3.3 .01 .00 .0 \mathrm{~A}$ \\
\hline Plant uptake & $3.3 .02 .01 .0 \mathrm{~A}$ \\
\hline Animal uptake & $3.3 .02 .02 .0 \mathrm{~A}$ \\
\hline Contaminated non-food products and exposure & $3.3 .03 .01 .0 \mathrm{~A}$ \\
\hline Ingestion & $3.3 .04 .01 .0 \mathrm{~A}$ \\
\hline Inhalation & $3.3 .04 .02 .0 \mathrm{~A}$ \\
\hline External exposure & $3.3 .04 .03 .0 \mathrm{~A}$ \\
\hline Radiation doses & $3.3 .05 .01 .0 \mathrm{~A}$ \\
\hline Radon and radon daughter exposure & $3.3 .08 .00 .0 \mathrm{~A}$ \\
\hline
\end{tabular}

Source: DTN: MO0501SEPFEPLA.001 [DIRS 172601]. 
Table 1-2. Features, Events, and Processes Related to Inhalation Dose Factors for the Eruption Phase

\begin{tabular}{|c|c|c|}
\hline FEP Number ${ }^{a}$ & FEP Name ${ }^{a}$ & Description of FEP Relationship to Inhalation Dose Factors \\
\hline 1.2.04.07.0A & Ashfall & $\begin{array}{l}\text { Volcanic ash is the source of inhalation exposure during volcanic } \\
\text { eruption. Inhalation dose is proportional to activity concentration in } \\
\text { the airborne ash (Equation } 6.3-2 \text { ). }\end{array}$ \\
\hline $2.4 .01 .00 .0 \mathrm{~A}$ & $\begin{array}{l}\text { Human characteristics } \\
\text { (physiology, metabolișm) }\end{array}$ & $\begin{array}{l}\text { Physiology and metabolism of the human receptor were } \\
\text { considered in developing the values of breathing rates and dose } \\
\text { coefficients for inhalation, which are used as input to calculate the } \\
\text { values of dose factors (Equation 6.3-3). }\end{array}$ \\
\hline 2.4.04.01.0A & Human lifestyle & $\begin{array}{l}\text { Lifestyles and characteristics of people living in Amargosa Valley } \\
\text { were considered in developing the values of population proportions } \\
\text { and exposure times, which are used as input to calculate the } \\
\text { values of dose factors (Equation 6.3-3). }\end{array}$ \\
\hline $2.4 .07 .00 .0 \mathrm{~A}$ & Dwellings & $\begin{array}{l}\text { Dwellings produce mitigating effect on inhalation dose, which is } \\
\text { considered in the value of the indoor reduction factor for activity } \\
\text { concentration in air, which is used to calculate the values of dose } \\
\text { factors (Equation } 6.3-3 \text { ). }\end{array}$ \\
\hline $3.1 .01 .01 .0 \mathrm{~A}$ & $\begin{array}{l}\text { Radioactive decay and } \\
\text { ingrowth }\end{array}$ & $\begin{array}{l}\text { Contribution from long-lived and short-lived decay products of } \\
\text { primary radionuclides is included in the values of dose factors, } \\
\text { primarily through the use of effective dose coefficients for } \\
\text { inhalation (Tables } 6.3-1 \text { and } 6.3-2 \text { ) and also through including long- } \\
\text { lived decay products in the dose factor for a primary radionuclide. }\end{array}$ \\
\hline 3.2.10.00.0A & $\begin{array}{l}\text { Atmospheric transport of } \\
\text { contaminants }\end{array}$ & $\begin{array}{l}\text { Although not directly included in the development of the dose } \\
\text { factors, atmospheric transport of radionuclides and the process of } \\
\text { deposition of contaminated ash on the ground are inherently } \\
\text { included in the calculation of the inhalation dose associated with } \\
\text { the eruption phase (Section } 6.3 \text { ). }\end{array}$ \\
\hline 3.3.04.02.0A & Inhalation & $\begin{array}{l}\text { Dose factors are used in evaluating exposure of the receptor } \\
\text { arising from inhalation of contaminated resuspended particles } \\
\text { (Equation } 6.3-2 \text { ). }\end{array}$ \\
\hline 3.3.05.01.0A & Radiation doses & $\begin{array}{l}\text { Radiation doses arising from inhalation exposure during volcanic } \\
\text { eruption will be evaluated in TSPA using dose factors developed in } \\
\text { this analysis (Section } 6.3 \text { ). }\end{array}$ \\
\hline
\end{tabular}


INTENTIONALLY LEFT BLANK 


\section{QUALITY ASSURANCE}

This analysis involved development of data to support performance assessment, as identified in the technical work plan (BSC 2005 [DIRS 172782]), and thus, it was a quality affecting activity in accordance with LP-2.29Q-BSC, Planning for Science Activities. Approved quality assurance (QA) procedures identified in the TWP (BSC 2005 [DIRS 172782], Section 4) were used to conduct and document the activities described in this report. Specifically, the procedure governing development of this document was LP-SIII.9Q-BSC, Scientific Analyses. Electronic data used in this analysis were controlled in accordance with methods specified in the TWP (BSC 2005 [DIRS 172782], Section 8).

The natural barriers and items identified in the Q-List (BSC 2005 [DIRS 171190]) are not pertinent to this analysis. A Safety Category per AP-2.22Q, Classification Analyses and Maintenance of the Q-List, is not applicable. 
INTENTIONALLY LEFT BLANK 


\section{USE OF SOFTWARE}

This analysis was performed using the verified and validated model, ERMYN, described in the Biosphere Model Report (BSC 2004 [DIRS 169460]). The model files were obtained from the Model Warehouse (DTN: MO0306MWDBGSMF.001 [DIRS 163816]). The model runs were conducted using the GoldSim Probabilistic Simulation Environment, a graphical, object-oriented computer program for carrying out dynamic, probabilistic simulations (GoldSim Technology Group 2003 [DIRS 166226]).

The analysis was conducted using the following two versions of software qualified under the Office of Civilian Radioactive Waste Management, QA program, for use on the Yucca Mountain Project:

- GoldSim V.8.01 Service Pack 4, STN: 10344-8.01SP4-00, Windows 2000 (BSC 2004 [DIRS 169695])

- GoldSim V.8.02.300, STN: 10344-8.02-03, Windows 2000 (BSC 2004 [DIRS 172588])

The software was appropriate for the application of running ERMYN model, which was implemented using GoldSim software, as described in the Biosphere Model Report (BSC 2004 [DIRS 169460], Section 6.9). GoldSim was used within the range of validation in accordance with procedure LP-SI.11Q-BSC, Software Management.

Both versions of GoldSim were installed by Software Configuration Management on a DELL Precision Workstation 530 computer and run under the Windows 2000 operating system. The GoldSim files generated in this analysis are shown in Appendix A. The original verification of the model was performed using GoldSim V.7.50.100, STN: 10344-7.50-100-00 (BSC 2003 [DIRS 161572]). Verification of the model for the use with GoldSim V.8.01 Service Pack 4 (BSC 2004 [DIRS 169695]) and V.8.02.300 (BSC 2004 [DIRS 172588]) is described in Appendix C.

In addition, the commercial off-the-shelf product Microsoft ${ }^{\circledR}$ Excel 2000 (Version 9.0.3821 SR-1) was used for data reduction. Standard functions of that software were used to calculate values included in tables in Section 6. The use of those functions, including formulas or algorithms, inputs, and outputs are described in Appendix B. 
INTENTIONALLY LEFT BLANK 


\section{INPUTS}

\subsection{DIRECT INPUTS}

\subsubsection{Direct Inputs Used for Calculation of Biosphere Dose Conversion Factors}

The inputs to this analysis are listed in Tables 4.1-1 to 4.1-5. Input parameter values and distributions were generated specifically for the biosphere model input and are appropriate for their intended use. The appropriateness for the intended use in this analysis and uncertainty in the input parameters are discussed in detail in the individual analysis reports that document parameter development (BSC 2005 [DIRS 172827]; BSC 2004 [DIRS 169672]; BSC 2004 [DIRS 169673]; BSC 2004 [DIRS 169458]; and BSC 2004 [DIRS 169459]).

The parameters were used as input for the ERMYN model for the volcanic ash exposure scenario, which is included in DTN: MO0306MWDBGSMF.001 [DIRS 163816]. The half-lives and branching fractions for radionuclides included in the biosphere model are listed in Table 4.1-2. The dose coefficients for inhalation and ingestion consistent with ICRP 30 dosimetric methods (ICRP 1979 [DIRS 110386]; ICRP 1980 [DIRS 110351]; ICRP 1981 [DIRS 110352]) were published in Federal Guidance Report No. 11 (FGR 11) (Eckerman et al. 1988 [DIRS 101069]) and are shown in Table 4.1-3. The dose coefficients tabulated in FGR 11 allow calculating committed effective dose equivalent, as defined in 10 CFR 63.2 [DIRS 173164]. The dose coefficients for inhalation and ingestion from ICRP 72 (ICRP 1996 [DIRS 152446]) are listed in Table 4.1-4. ICRP Publication 72 (ICRP 1996 [DIRS 152446]) contains the most current compilation of dose coefficients for inhalation and ingestion of radionuclides.

The dose coefficients for exposure to contaminated soil consistent with ICRP 30 and ICRP 72 dosimetric methods are shown in Table 4.1-5. Both sets are based on dose coefficients for exposure to contaminated soil organs developed in Federal Guidance Report No. 12 (FGR 12) (Eckerman and Ryman 1993 [DIRS 107684]). The difference is that the effective dose equivalent for the ICRP 30-based methodology is calculated using tissue weighting factors from ICRP Publication 26 (ICRP 1977 [DIRS 101075]), while the effective dose for the ICRP 72based methodology is calculated using tissue weighting factors from ICRP Publication 60 (ICRP 1991 [DIRS 101836]).

Additional inputs were taken from the textbook references (sources of established fact data) to help with the statistical analysis of the results. Specifically, Steel and Torrie (1980 [DIRS 150857], pp. 278 to 279) was used as a source of statistical tests of hypotheses and Lide and Frederikse (1997 [DIRS 103178], p. A-105) was used as a source of percentage points for the Student-t distribution.

For brevity, the parameters consistent with the ICRP 30 dosimetric methods are referred to as ICRP 30-based while the parameters consistent with the ICRP 72 dosimetric methods are referred to as ICRP 72-based.

The above direct inputs are used in Section 6.2. 


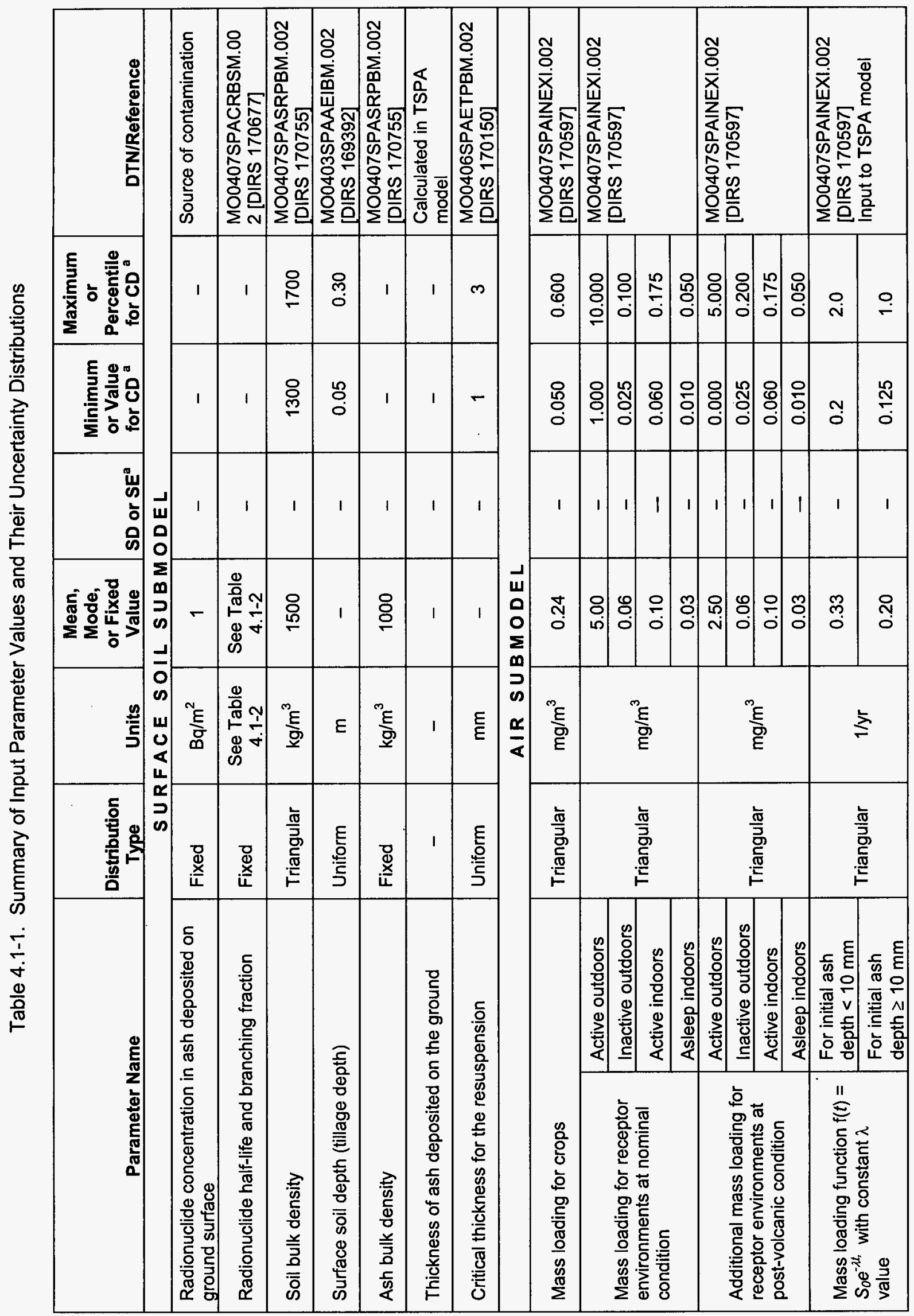




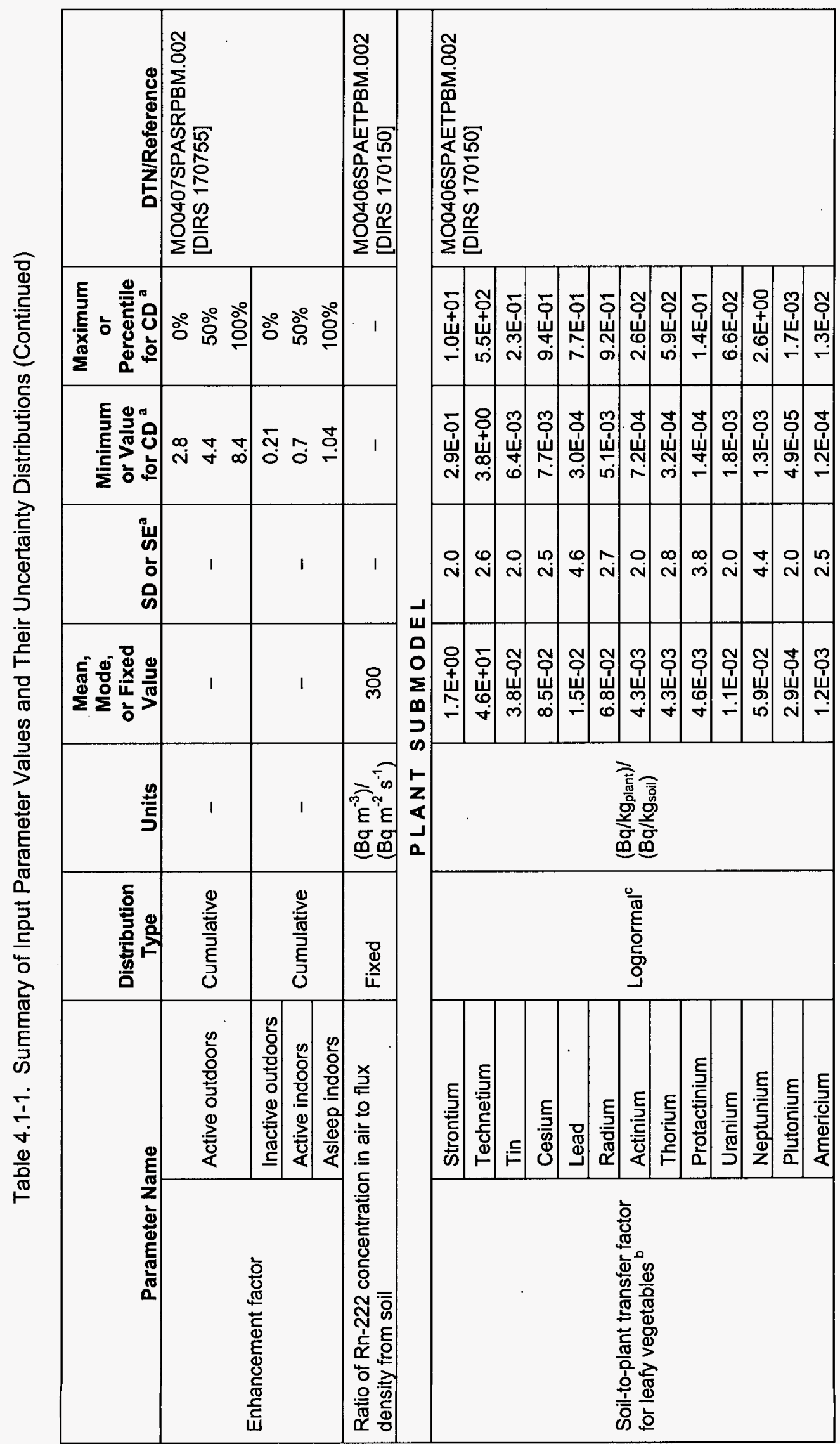




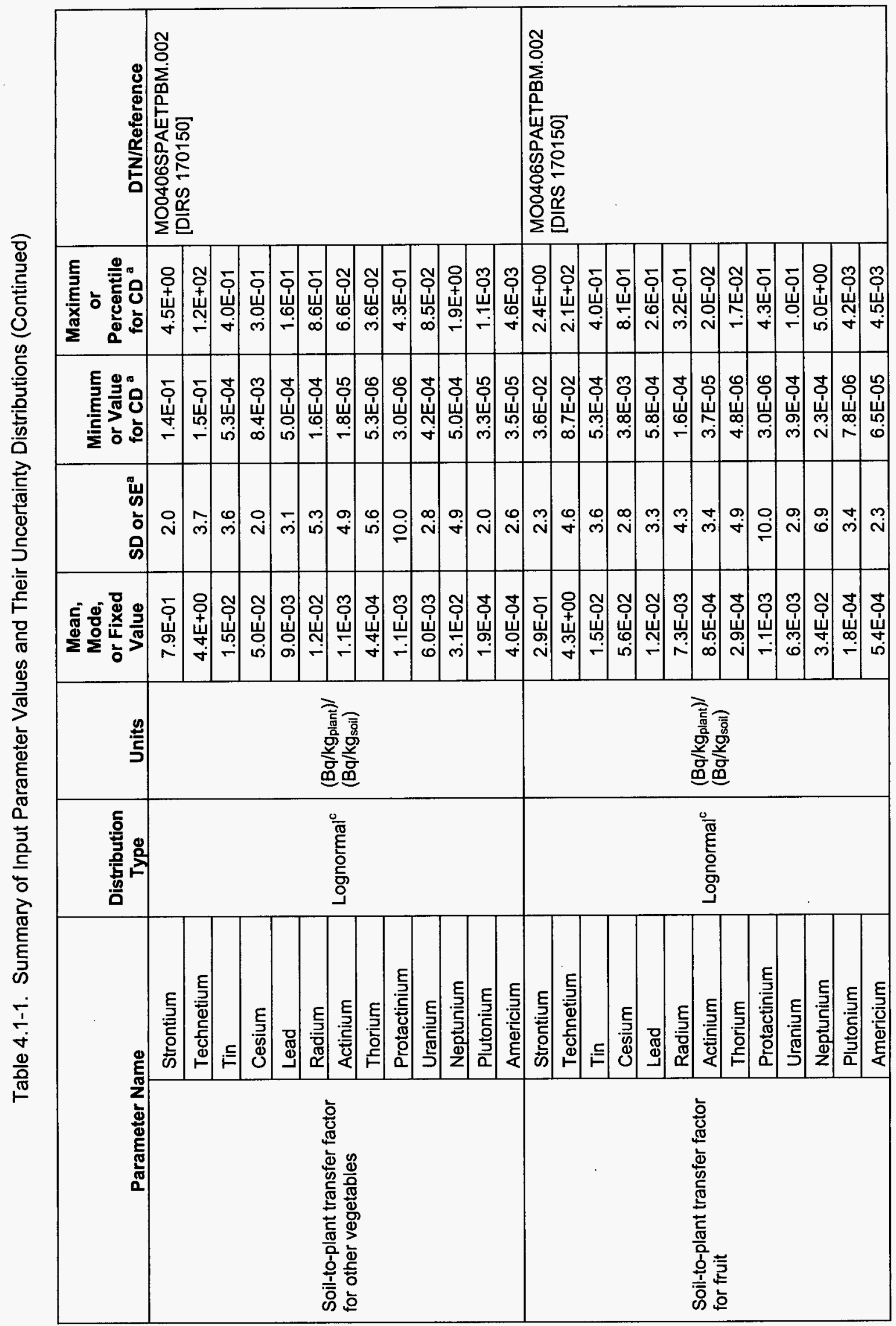




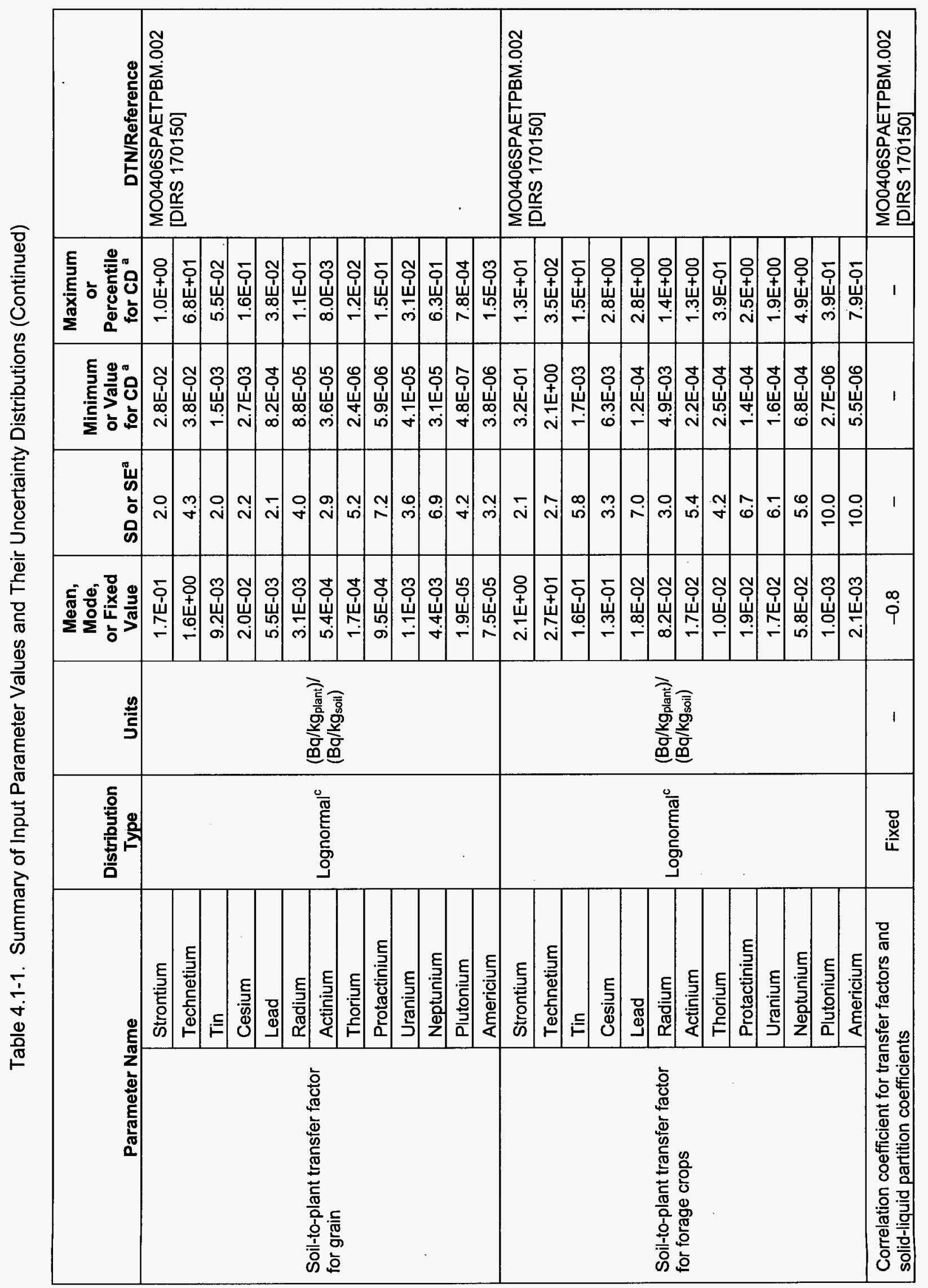




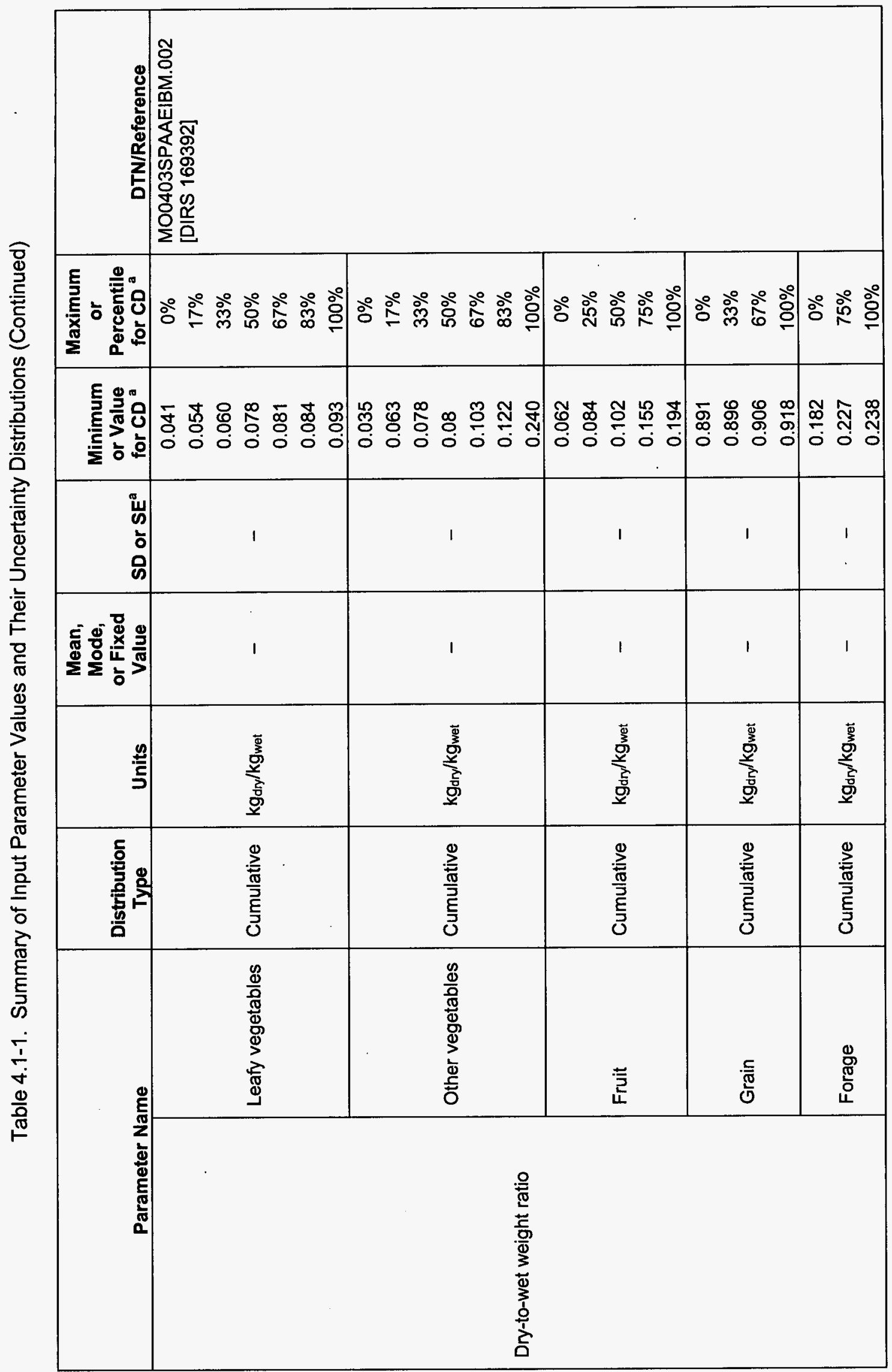




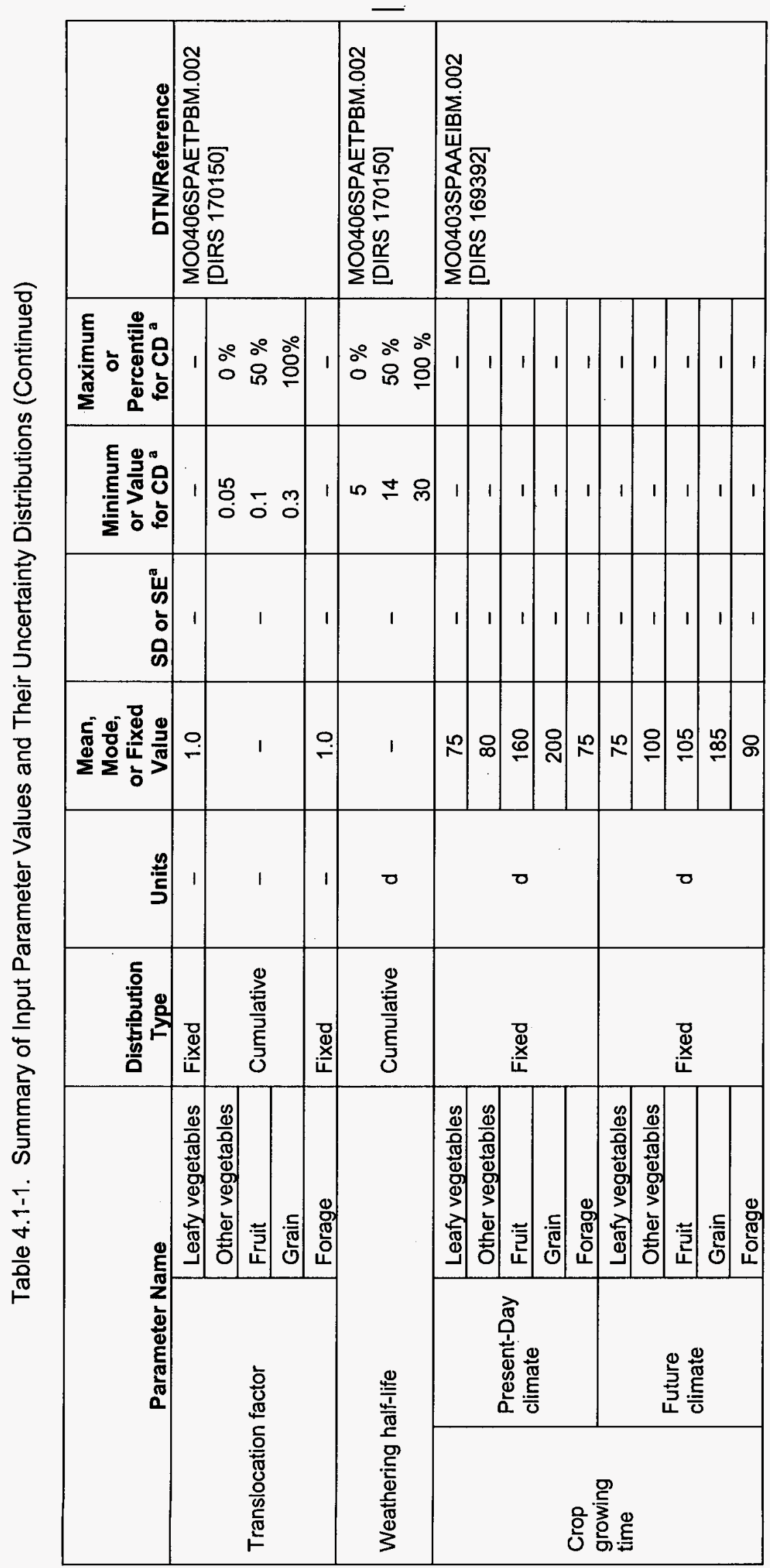




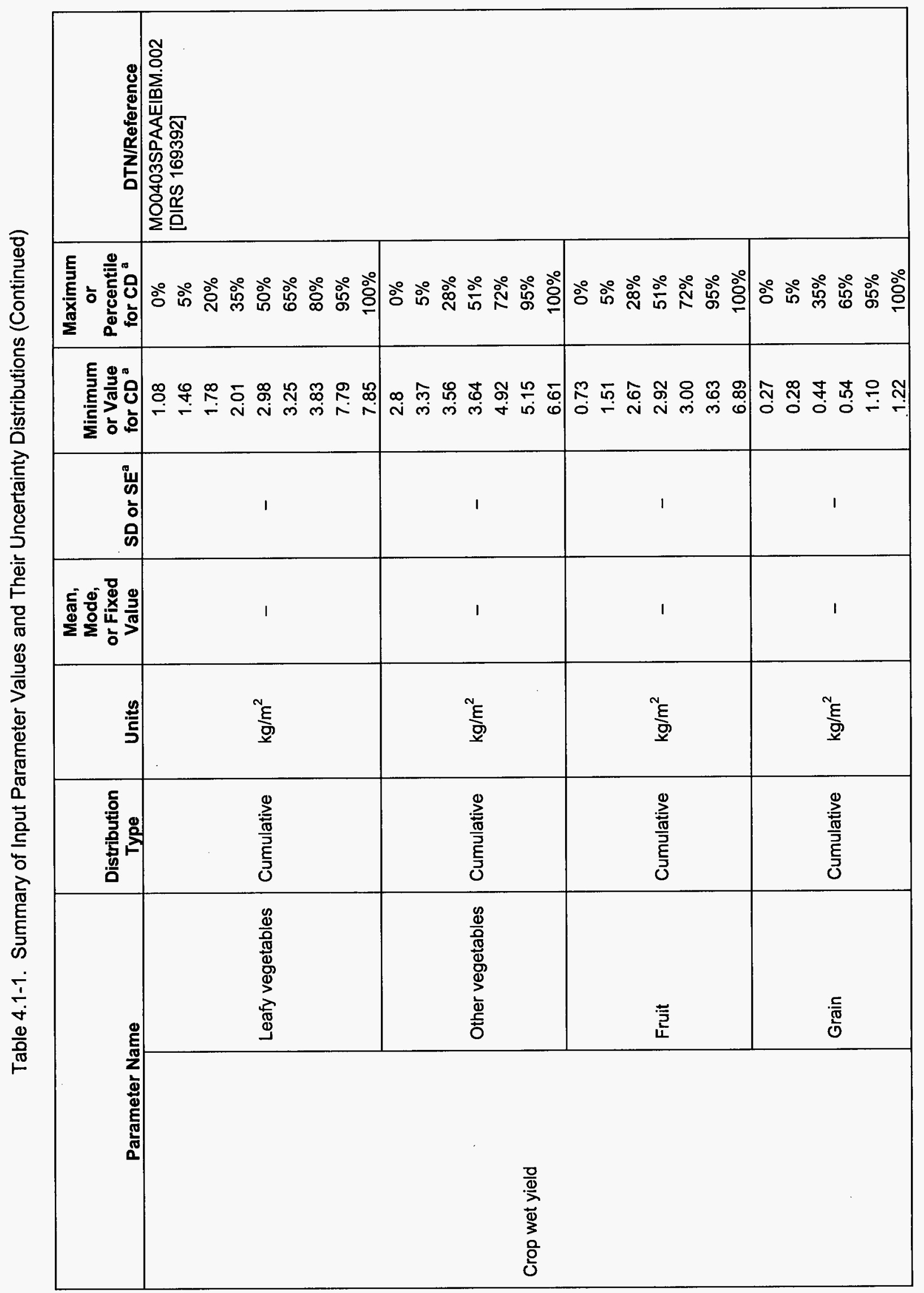




\begin{tabular}{|c|c|c|c|c|}
\hline 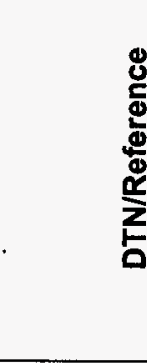 & 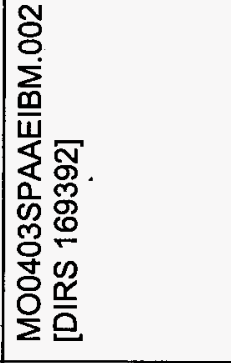 & 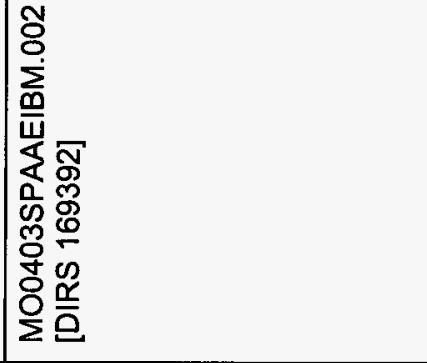 & & \\
\hline 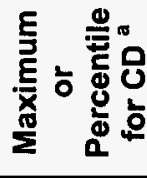 & 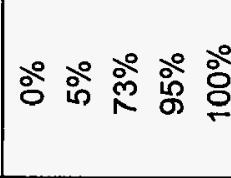 & 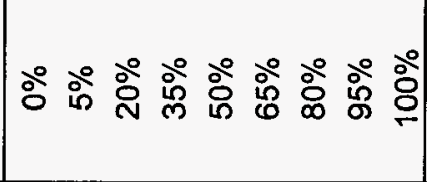 & 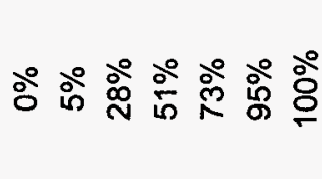 & 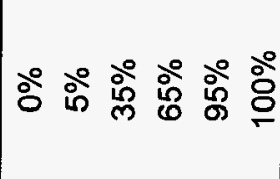 \\
\hline 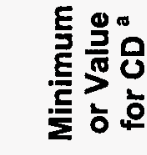 & 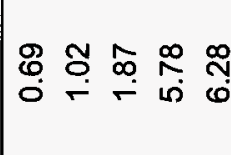 & 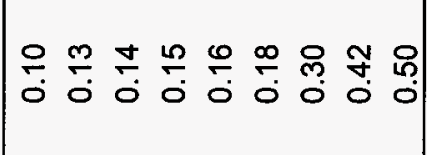 & 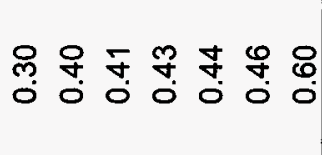 & 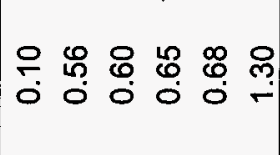 \\
\hline 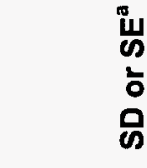 & 1 & 1 & 1 & 1 \\
\hline 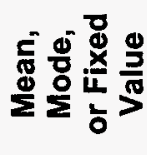 & 1 & 1 & 1 & 1 \\
\hline$\frac{\mathscr{s}}{5}$ & $\stackrel{n}{\frac{N}{9}}$ & 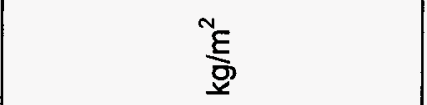 & $\stackrel{\text { ₹E}}{\underline{\partial}}$ & 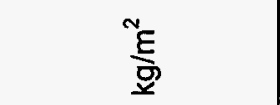 \\
\hline 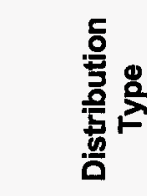 & 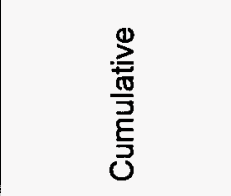 & 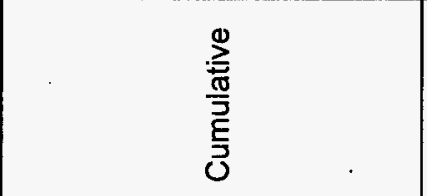 & 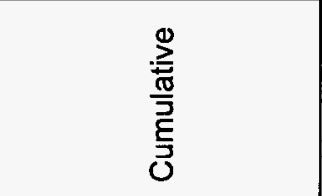 & 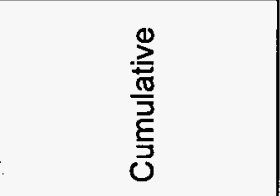 \\
\hline \multirow{2}{*}{ 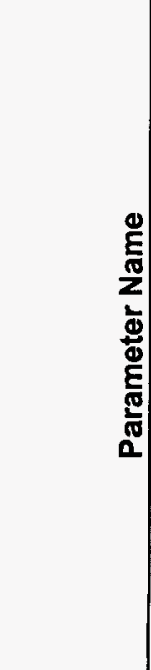 } & 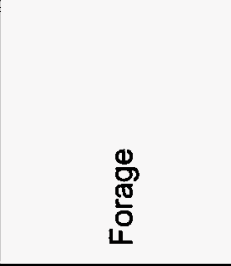 & 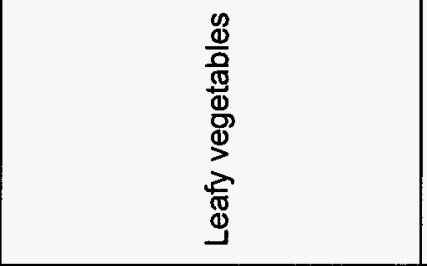 & 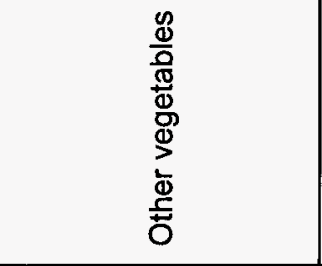 & 蛋 \\
\hline & 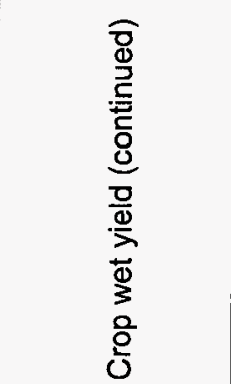 & \multicolumn{3}{|c|}{ 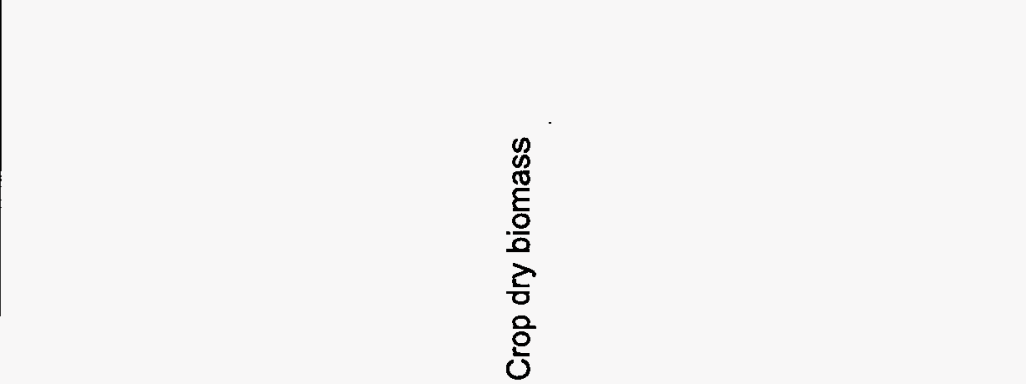 } \\
\hline
\end{tabular}




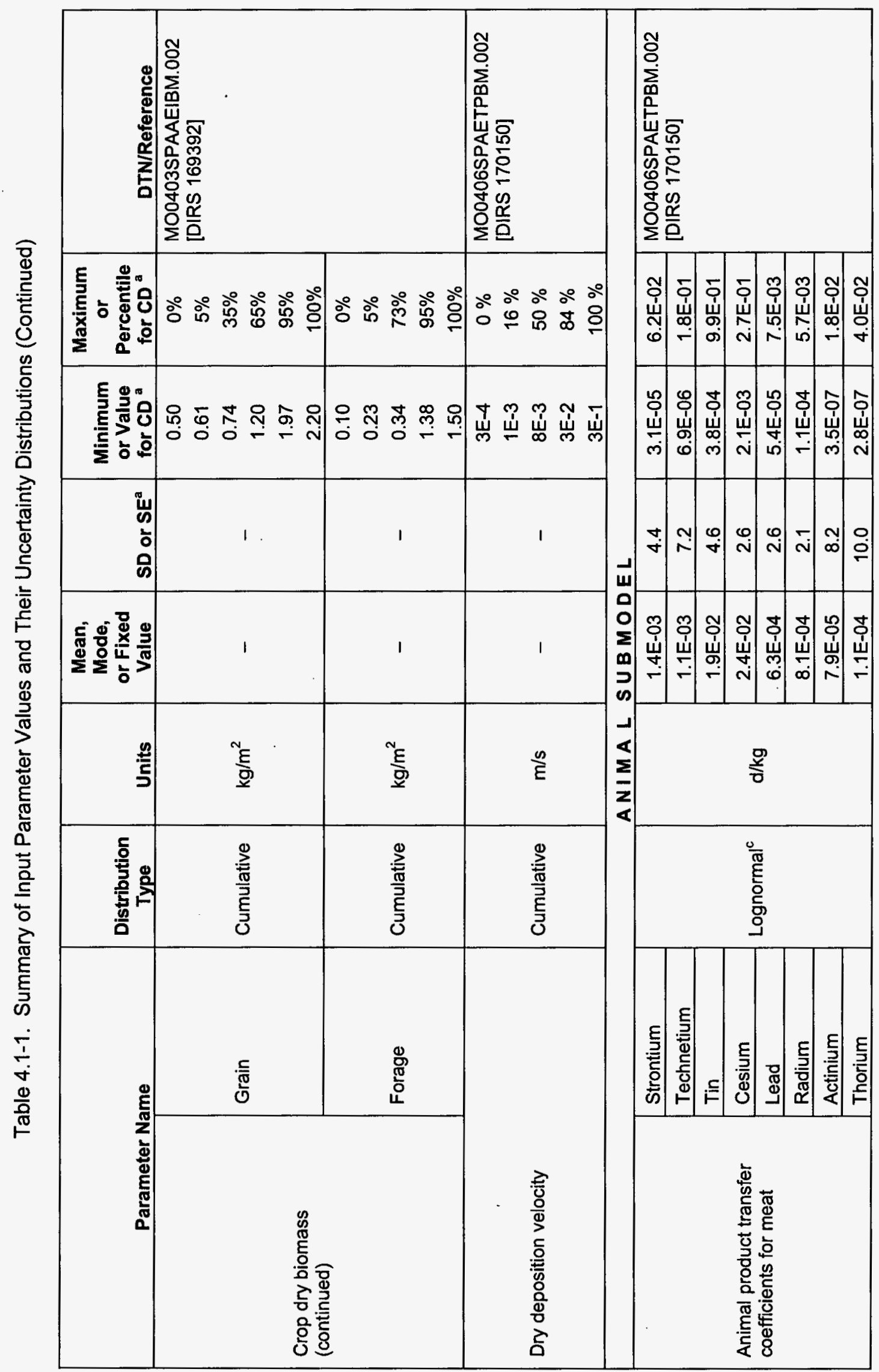




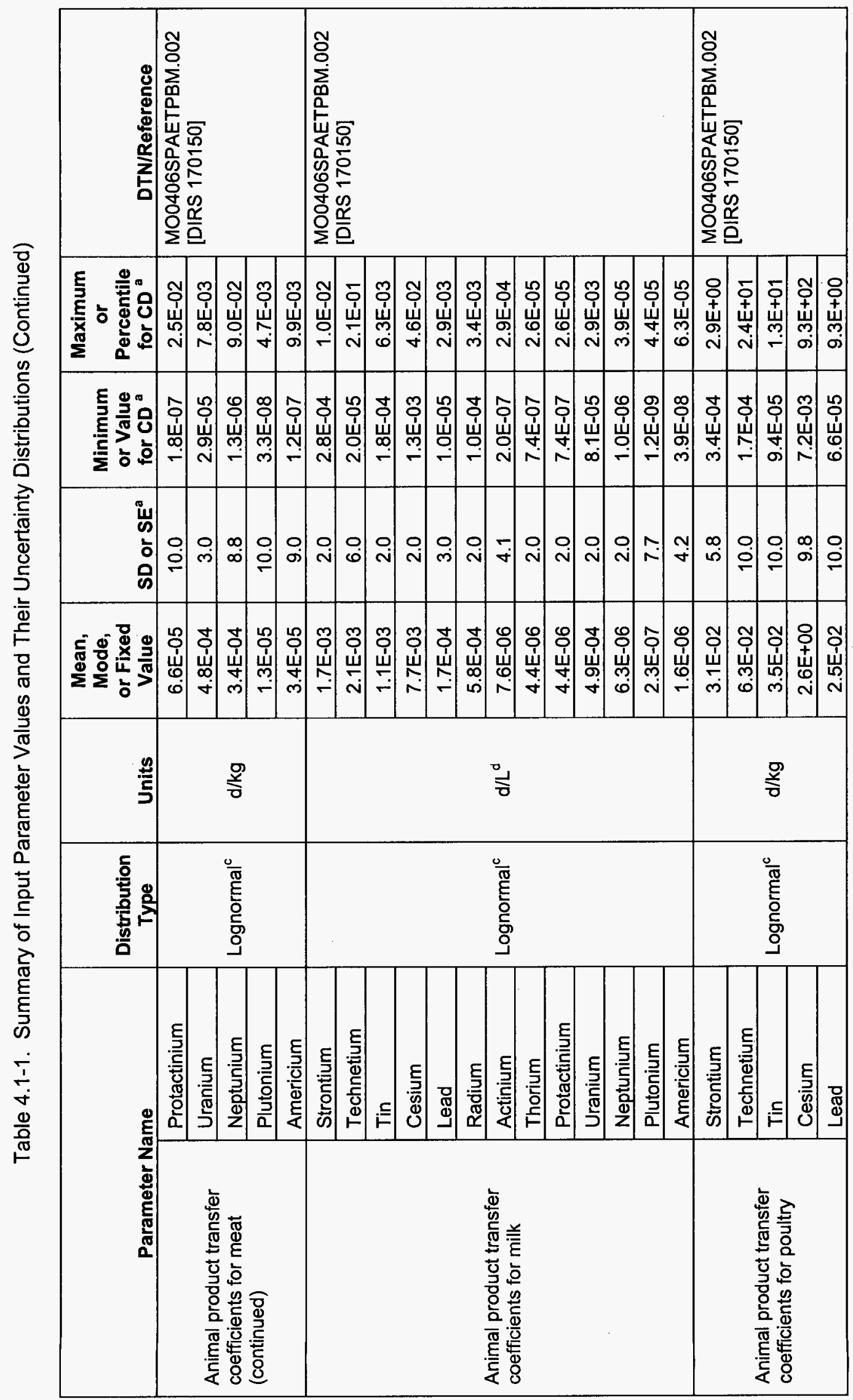




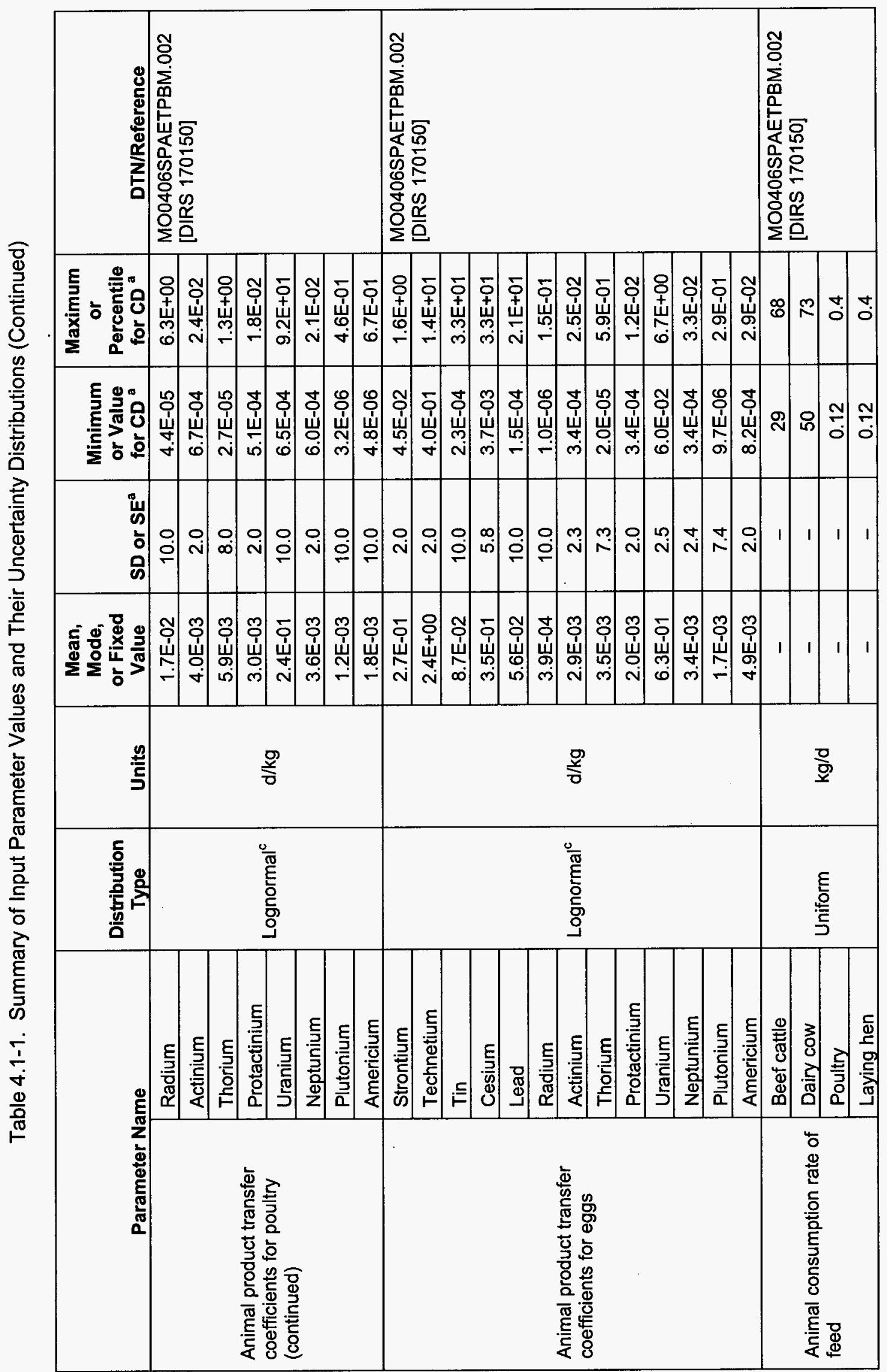




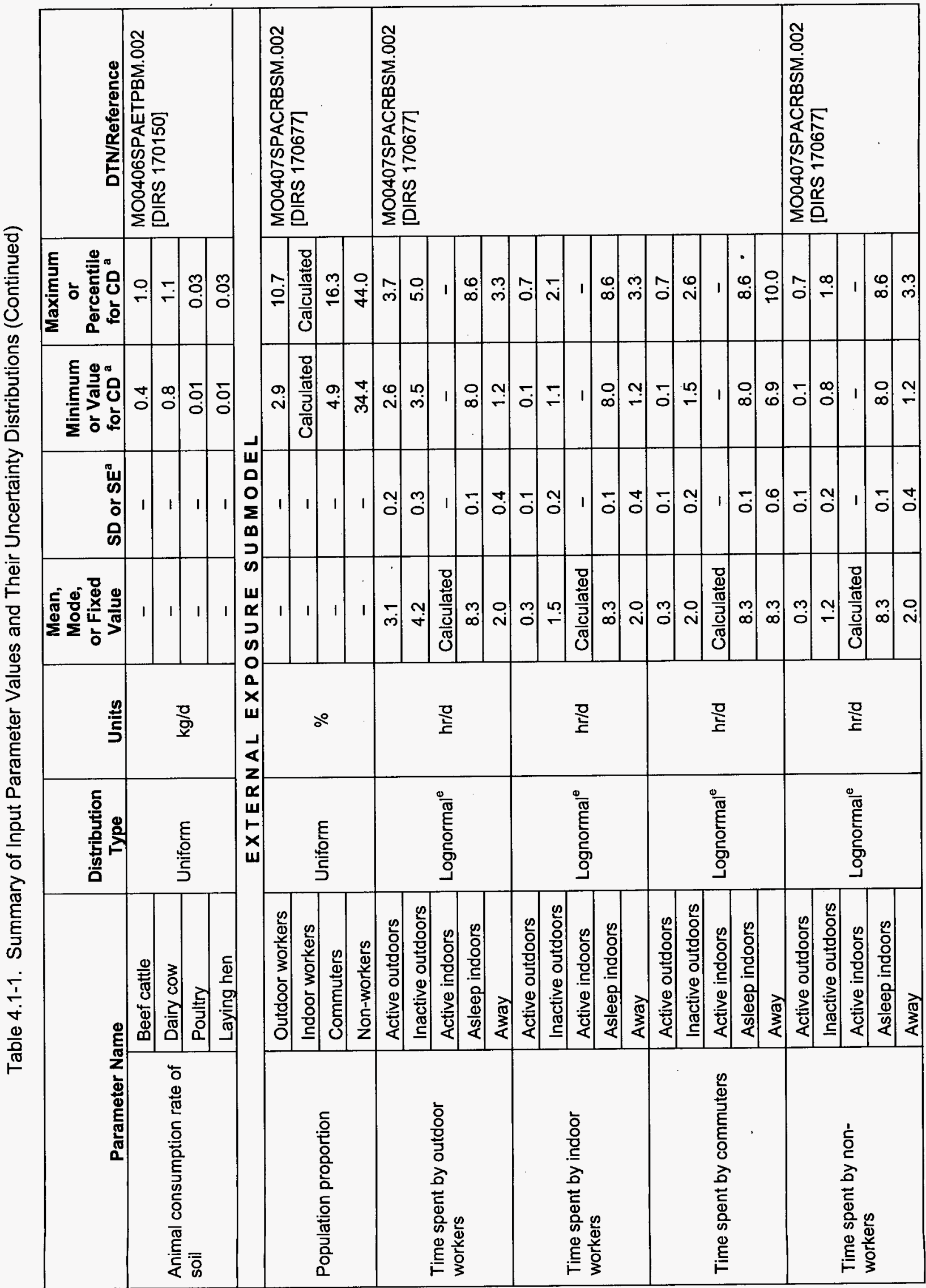




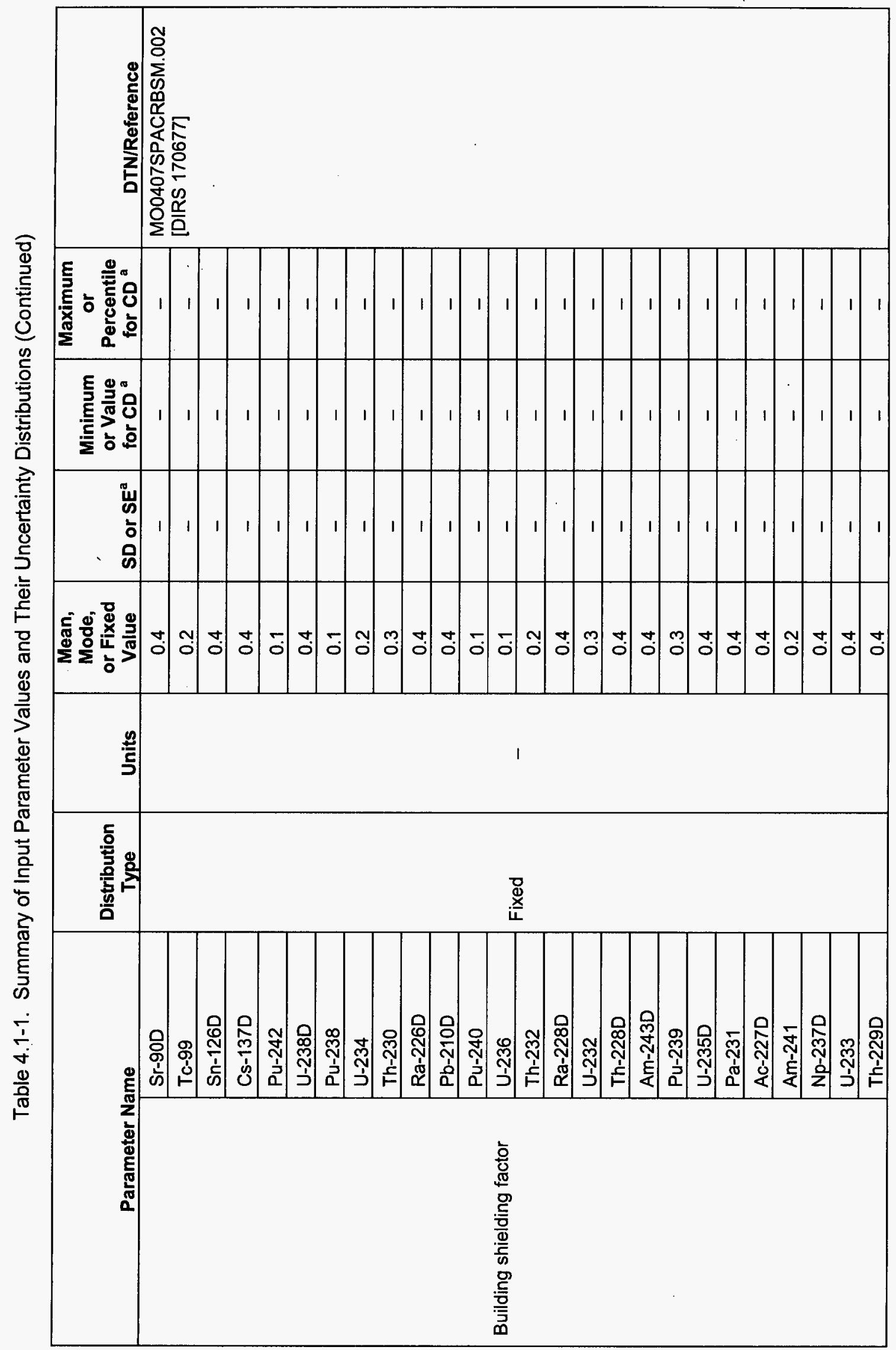




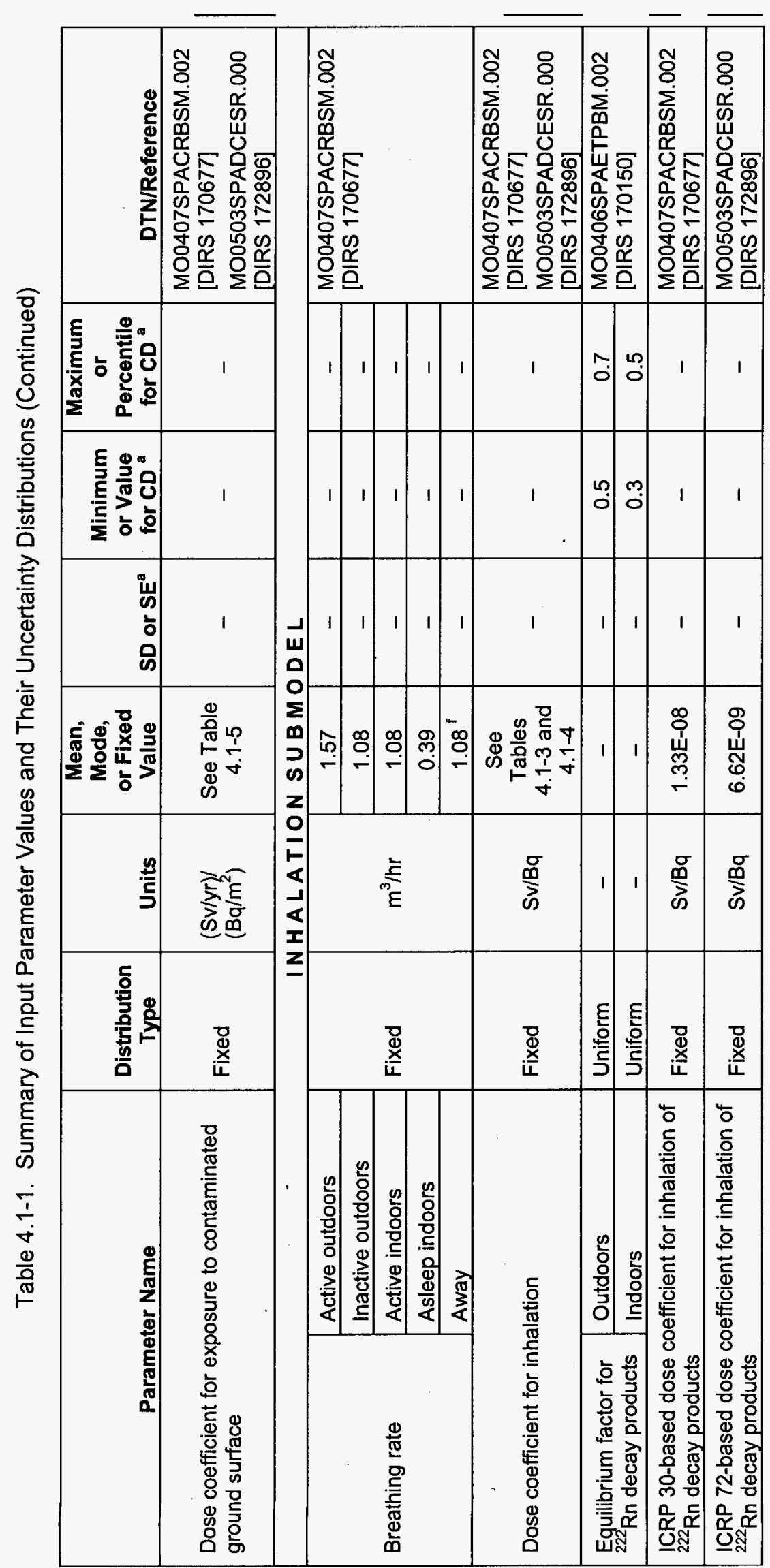




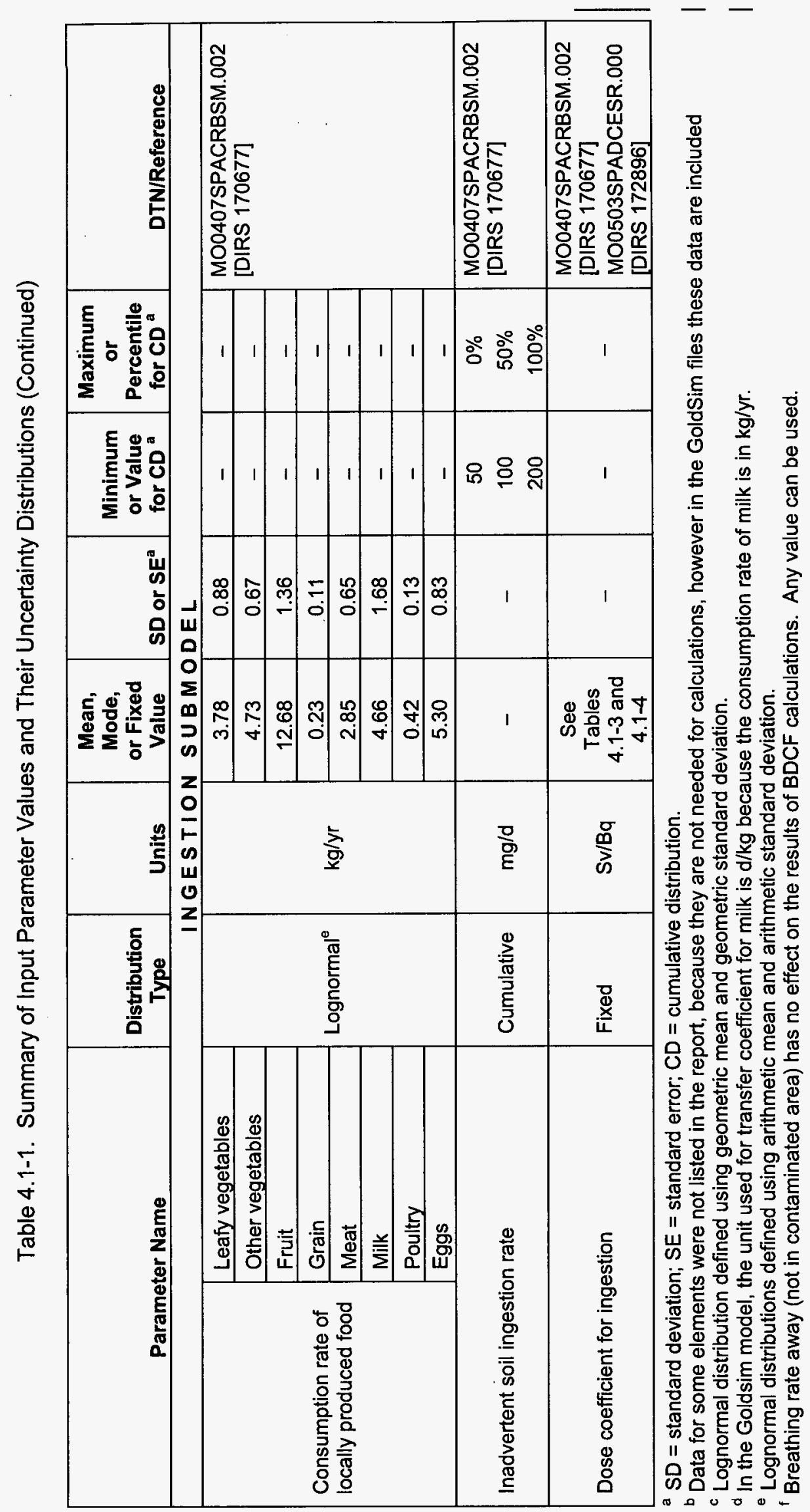


Table 4.1-2. Primary Radionuclides and Their Decay Products Included in the Biosphere Model for the Volcanic Ash Exposure Scenario

\begin{tabular}{|c|c|c|c|}
\hline Primary Radionuclide & Short-lived Decay Product ${ }^{a}$ & Branching Fraction, $\%$ & Half-life \\
\hline \multirow[t]{2}{*}{ Strontium-90 } & & 100 & $2.912 E+01 y r$ \\
\hline & Yttrium-90 & 100 & $6.40 \mathrm{E}+01 \mathrm{hr}$ \\
\hline Technetium-99 & & 100 & $2.13 \mathrm{E}+05 \mathrm{yr}$ \\
\hline \multirow[t]{3}{*}{ Tin-126 } & & 100 & $1.0 \mathrm{E}+05 \mathrm{yr}$ \\
\hline & Antimony-126m & 100 & $1.90 \mathrm{E}+01 \mathrm{~min}$ \\
\hline & Antimony-126 & 14 & 1.24E+01d \\
\hline \multirow[t]{2}{*}{ Cesium-137 } & & 100 & $3.00 \mathrm{E}+01 \mathrm{yr}$ \\
\hline & Barium-137m & 94.60 & $2.552 E+00 \mathrm{~min}$ \\
\hline \multicolumn{4}{|c|}{ Thorium Series (4n) } \\
\hline Plutonium-240 & & 100 & $6.537 E+03 y r$ \\
\hline Uranium-236 & & 100 & $2.3415 \mathrm{E}+07 \mathrm{yr}$ \\
\hline Thorium-232 & & 100 & $1.405 \mathrm{E}+10 \mathrm{yr}$ \\
\hline \multirow[t]{2}{*}{ Radium-228 } & & 100 & $5.75 \mathrm{E}+00 \mathrm{yr}$ \\
\hline & Actinium-228 & 100 & $6.13 \mathrm{E}+00 \mathrm{hr}$ \\
\hline Uranium-232 & & 100 & $7.2 \mathrm{E}+01 \mathrm{yr}$ \\
\hline \multirow[t]{8}{*}{ Thorium-228 } & & 100 & $1.9131 \mathrm{E}+00 \mathrm{yr}$ \\
\hline & Radium-224 & 100 & $3.66 \mathrm{E}+00 \mathrm{~d}$ \\
\hline & Radon-220 & 100 & $5.56 \mathrm{E}+01 \mathrm{~s}$ \\
\hline & Polonium-216 & 100 & $1.5 \mathrm{E}-01 \mathrm{~s}$ \\
\hline & Lead-212 & 100 & $1.064 \mathrm{E}+01 \mathrm{hr}$ \\
\hline & Bismuth-212 & 100 & $6.055 \mathrm{E}+01 \mathrm{~min}$ \\
\hline & Polonium-212 & 64.07 & $3.05 \mathrm{E}-07 \mathrm{~s}$ \\
\hline & Thallium-208 & 35.93 & $3.07 \mathrm{E}+00 \mathrm{~min}$ \\
\hline \multicolumn{4}{|c|}{ Neptunium Series $(4 n+1)$} \\
\hline Americium-241 & & 100 & $4.322 \mathrm{E}+02 \mathrm{yr}$ \\
\hline \multirow[t]{2}{*}{ Neptunium-237 } & & 100 & $2.14 \mathrm{E}+06 \mathrm{yr}$ \\
\hline & Protactinium-233 & 100 & $2.70 E+01 d$ \\
\hline Uranium-233 & & 100 & $1.585 \mathrm{E}+05 \mathrm{yr}$ \\
\hline \multirow[t]{9}{*}{ Thorium-229 } & & 100 & $7.340 \mathrm{E}+03 \mathrm{yr}$ \\
\hline & Radium-225 & 100 & $1.48 \mathrm{E}+01 \mathrm{~d}$ \\
\hline & Actinium-225 & 100 & $1.00 E+01 d$ \\
\hline & Francium-221 & 100 & $4.8 \mathrm{E}+00 \mathrm{~min}$ \\
\hline & Astatine-217 & 100 & $3.23 \mathrm{E}-02 \mathrm{~s}$ \\
\hline & Bismuth-213 & 100 & $4.565 \mathrm{E}+01 \mathrm{~min}$ \\
\hline & Polonium-213 & 97.84 & $4.2 \mathrm{E}-06 \mathrm{~s}$ \\
\hline & Thallium-209 & 2.16 & $2.20 \mathrm{E}+00 \mathrm{~min}$ \\
\hline & Lead-209 & 100 & $3.253 \mathrm{E}+00 \mathrm{hr}$ \\
\hline
\end{tabular}


Table 4.1-2. Primary Radionuclides and Their Decay Products Included in the Biosphere Model for the Volcanic Ash Exposure Scenario (Continued)

\begin{tabular}{|c|c|c|c|}
\hline Primary Radionuclide & Short-lived Decay Product ${ }^{\text {a }}$ & Branching Fraction, $\%$ & Half-life \\
\hline \multicolumn{4}{|c|}{ Uranium Series $(4 n+2)$} \\
\hline Plutonium-242 & & 100 & $3.763 E+05$ yr \\
\hline \multirow[t]{4}{*}{ Uranium-238 } & & 100 & $4.468 \mathrm{E}+09 \mathrm{yr}$ \\
\hline & Thorium-234 & 100 & $2.410 E+01 d$ \\
\hline & Protactinium-234m & 99.80 & 1.17E+00 min \\
\hline & Protactinium-234 & 0.33 & $6.70 \mathrm{E}+00 \mathrm{hr}$ \\
\hline Plutonium-238 & & 100 & $8.774 \mathrm{E}+01 \mathrm{yr}$ \\
\hline Uranium-234 & & 100 & $2.445 \mathrm{E}+05 \mathrm{yr}$ \\
\hline Thorium-230 & & 100 & $7.7 \mathrm{E}+04 \mathrm{yr}$ \\
\hline \multirow[t]{8}{*}{ Radium-226 } & & 100 & $1.600 \mathrm{E}+03 \mathrm{yr}$ \\
\hline & Radon-222 & 100 & $3.8235 E+00 d$ \\
\hline & Polonium-218 & 100 & $3.05 \mathrm{E}+00 \mathrm{~min}$ \\
\hline & Lead-214 & 99.98 & $2.68 \mathrm{E}+01 \mathrm{~min}$ \\
\hline & Astatine-218 & 0.02 & $2 . \mathrm{E}+00 \mathrm{~s}$ \\
\hline & Bismuth-214 & 100 & $1.99 \mathrm{E}+01 \mathrm{~min}$ \\
\hline & Polonium-214 & 99.98 & $1.643 \mathrm{E}-04 \mathrm{~s}$ \\
\hline & Thallium-210 & 0.02 & $1.3 E+00 \min ^{b}$ \\
\hline \multirow[t]{3}{*}{ Lead-210 } & & 100 & $2.23 \mathrm{E}+01 \mathrm{yr}$ \\
\hline & Bismuth-210 & 100 & $5.012 \mathrm{E}+00 \mathrm{~d}$ \\
\hline & Polonium-210 & 100 & $1.3838 E+02 d$ \\
\hline \multicolumn{4}{|c|}{ Actinium Series $(4 n+3)$} \\
\hline \multirow[t]{2}{*}{ Americium-243 } & & 100 & $7.380 \mathrm{E}+03 \mathrm{yr}$ \\
\hline & Neptunium-239 & 100 & $2.355 E+00 d$ \\
\hline Plutonium-239 & & 100 & $2.4065 \mathrm{E}+04 \mathrm{yr}$ \\
\hline \multirow[t]{2}{*}{ Uranium-235 } & & 100 & $7.038 \mathrm{E}+08 \mathrm{yr}$ \\
\hline & Thorium-231 & 100 & $2.552 \mathrm{E}+01 \mathrm{hr}$ \\
\hline Protactinium-231 & & 100 & $3.276 \mathrm{E}+04 \mathrm{yr}$ \\
\hline \multirow[t]{10}{*}{ Actinium-227 } & & 100 & $2.1773 \mathrm{E}+01 \mathrm{yr}$ \\
\hline & Thorium-227 & 98.62 & $1.8718 \mathrm{E}+01 \mathrm{~d}$ \\
\hline & Francium-223 & 1.38 & $2.18 \mathrm{E}+01 \mathrm{~min}$ \\
\hline & Radium-223 & 100 & $1.1434 E+01 d$ \\
\hline & Radon-219 & 100 & $3.96 \mathrm{E}+00 \mathrm{~s}$ \\
\hline & Polonium-215 & 100 & $1.78 \mathrm{E}-03 \mathrm{~s}$ \\
\hline & Lead-211 & 100 & $3.61 \mathrm{E}+01 \mathrm{~min}$ \\
\hline & Bismuth-211 & 100 & $2.14 \mathrm{E}+00 \mathrm{~min}$ \\
\hline & Thallium-207 & 99.72 & 4.77E+00 min \\
\hline & Polonium-211 & 0.28 & $5.16 \mathrm{E}-01 \mathrm{~s}$ \\
\hline
\end{tabular}

Source: MO0407SPACRBSM.002 [DIRS 170677].

a Short-lived decay products of primary radionuclides are assumed to be in secular equilibrium with their parents (Section 6.1.1). 
Table 4.1-3. Dose Coefficients for Inhalation and Ingestion of Radionuclides of Interest Based on ICRP 30 Dosimetric Methods

\begin{tabular}{|c|c|c|c|}
\hline \multirow[b]{2}{*}{ Primary Radionuclide } & \multirow{2}{*}{$\begin{array}{c}\text { Short-lived Decay } \\
\text { Product }\end{array}$} & \multicolumn{2}{|c|}{ Dose Coefficient (Sv/Bq) ${ }^{a}$} \\
\hline & & Inhalation & Ingestion \\
\hline \multirow[t]{2}{*}{ Strontium-90 } & & $6.47 \mathrm{E}-08$ & $3.85 \mathrm{E}-08$ \\
\hline & Yttrium-90 & $2.28 \mathrm{E}-09$ & 2.91E-09 \\
\hline Technetium-99 & & $2.25 \mathrm{E}-09$ & $3.95 \mathrm{E}-10$ \\
\hline \multirow[t]{3}{*}{ Tin-126 } & & $2.69 \mathrm{E}-08$ & 5.27E-09 \\
\hline & Antimony-126m & $9.17 \mathrm{E}-12$ & $2.54 E-11$ \\
\hline & Antimony-126 & 3.17E-09 & 2.89E-09 \\
\hline \multirow[t]{2}{*}{ Cesium-137 } & & 8.63E-09 & 1.35E-08 \\
\hline & Barium-137m & - & $=$ \\
\hline \multicolumn{4}{|c|}{ Thorium Series (4n) } \\
\hline Plutonium-240 & & 1.16E-04 & $9.56 \mathrm{E}-07$ \\
\hline Uranium-236 & & $3.39 \mathrm{E}-05$ & $7.26 \mathrm{E}-08$ \\
\hline Thorium-232 & & 4.43E-04 & 7.38E-07 \\
\hline \multirow[t]{2}{*}{ Radium-228 } & & 1.29E-06 & $3.88 \mathrm{E}-07$ \\
\hline & Actinium-228 & 8.33E-08 & $5.85 \mathrm{E}-10$ \\
\hline Uranium-232 & & $1.78 \mathrm{E}-04$ & $3.54 \mathrm{E}-07$ \\
\hline \multirow[t]{8}{*}{ Thorium-228 } & & $9.23 \mathrm{E}-05$ & 1.07E-07 \\
\hline & Radium-224 & 8.53E-07 & $9.89 \mathrm{E}-08$ \\
\hline & Radon-220 & - & $=$ \\
\hline & Polonium-216 & - & - \\
\hline & Lead-212 & $4.56 \mathrm{E}-08$ & $1.23 \mathrm{E}-08$ \\
\hline & Bismuth-212 & $5.83 \mathrm{E}-09$ & $2.87 \mathrm{E}-10$ \\
\hline & Polonium-212 & - & - \\
\hline & Thallium-208 & - & - \\
\hline \multicolumn{4}{|c|}{ Neptunium Series $(4 n+1)$} \\
\hline Americium-241 & & $1.20 \mathrm{E}-04$ & $9.84 E-07$ \\
\hline \multirow[t]{2}{*}{ Neptunium-237 } & & 1.46E-04 & 1.20E-06 \\
\hline & Protactinium-233 & $2.58 \mathrm{E}-09$ & $9.81 \mathrm{E}-10$ \\
\hline Uranium-233 & & $3.66 \mathrm{E}-05$ & $7.81 \mathrm{E}-08$ \\
\hline \multirow[t]{9}{*}{ Thorium-229 } & & $5.80 \mathrm{E}-04$ & $9.54 \mathrm{E}-07$ \\
\hline & Radium-225 & $2.10 \mathrm{E}-06$ & $1.04 \mathrm{E}-07$ \\
\hline & Actinium-225 & $2.92 \mathrm{E}-06$ & $3.00 \mathrm{E}-08$ \\
\hline & Francium-221 & - & - \\
\hline & Astatine-217 & - & - \\
\hline & Bismuth-213 & 4.63E-09 & $1.95 \mathrm{E}-10$ \\
\hline & Polonium-213 & - & - \\
\hline & Thallium-209 & - & - \\
\hline & Lead-209 & $2.56 \mathrm{E}-11$ & $5.75 E-11$ \\
\hline
\end{tabular}


Table 4.1-3. Dose Coefficients for Inhalation and Ingestion of Radionuclides of Interest Based on ICRP 30 Dosimetric Methods (Continued)

\begin{tabular}{|c|c|c|c|}
\hline \multirow[b]{2}{*}{ Primary Radionuclide } & \multirow{2}{*}{$\begin{array}{c}\text { Short-lived Decay } \\
\text { Product }\end{array}$} & \multicolumn{2}{|c|}{ Dose Coefficient (Sv/Bq) ${ }^{a}$} \\
\hline & & Inhalation & Ingestion \\
\hline \multicolumn{4}{|c|}{ Uranium Series $(4 n+2)$} \\
\hline Plutonium-242 & & 1.11E-04 & $9.08 \mathrm{E}-07$ \\
\hline \multirow[t]{4}{*}{ Uranium-238 } & & $3.20 \mathrm{E}-05$ & $6.88 \mathrm{E}-08$ \\
\hline & Thorium-234 & 9.47E-09 & $3.69 \mathrm{E}-09$ \\
\hline & Protactinium-234m & - & - \\
\hline & Protactinium-234 & $2.20 \mathrm{E}-10$ & $5.84 \mathrm{E}-10$ \\
\hline Plutonium-238 & & $1.06 \mathrm{E}-04$ & 8.65E-07 \\
\hline Uranium-234 & & $3.58 \mathrm{E}-05$ & 7.66E-08 \\
\hline Thorium-230 & & 8.80E-05 & $1.48 \mathrm{E}-07$ \\
\hline \multirow[t]{8}{*}{ Radium-226 } & & $2.32 E-06$ & $3.58 \mathrm{E}-07$ \\
\hline & Radon-222 & - & - \\
\hline & Polonium-218 & - & - \\
\hline & Lead-214 & 2.11E-09 & $1.69 \mathrm{E}-10$ \\
\hline & Astatine-218 & - & - \\
\hline & Bismuth-214 & 1.78E-09 & 7.64E-11 \\
\hline & Polonium-214 & - & - \\
\hline & Thallium-210 & - & - \\
\hline \multirow[t]{3}{*}{ Lead-210 } & & 3.67E-06 & $1.45 \mathrm{E}-06$ \\
\hline & Bismuth-210 & $5.29 \mathrm{E}-08$ & $1.73 \mathrm{E}-09$ \\
\hline & Polonium-210 & $2.54 \mathrm{E}-06$ & $5.14 \mathrm{E}-07$ \\
\hline \multicolumn{4}{|c|}{ Actinium Series $(4 n+3)$} \\
\hline \multirow[t]{2}{*}{ Americium-243 } & & $1.19 \mathrm{E}-04$ & 9.79E-07 \\
\hline & Neptunium-239 & $6.78 \mathrm{E}-10$ & 8.82E-10 \\
\hline Plutonium-239 & & 1.16E-04 & $9.56 \mathrm{E}-07$ \\
\hline \multirow[t]{2}{*}{ Uranium-235 } & & $3.32 E-05$ & $7.19 \mathrm{E}-08$ \\
\hline & Thorium-231 & $2.37 E-10$ & $3.65 \mathrm{E}-10$ \\
\hline Protactinium-231 & & 3.47E-04 & 2.86E-06 \\
\hline \multirow[t]{10}{*}{ Actinium-227 } & & $1.81 \mathrm{E}-03$ & $3.80 \mathrm{E}-06$ \\
\hline & Thorium-227 & 4.37E-06 & 1.03E-08 \\
\hline & Francium-223 & $1.68 \mathrm{E}-09$ & 2.33E-09 \\
\hline & Radium-223 & 2.12E-06 & 1.78E-07 \\
\hline & Radon-219 & - & - \\
\hline & Polonium-215 & - & - \\
\hline & Lead-211 & $2.35 \mathrm{E}-09$ & 1.42E-10 \\
\hline & Bismuth-211 & - & - \\
\hline & Thallium-207 & - & - \\
\hline & Polonium-211 & - & - \\
\hline
\end{tabular}

Source: MO0407SPACRBSM.002 [DIRS 170677].

Notes: $\quad{ }^{a}$ The source DTN uses the term dose conversion factor instead of dose coefficient.

$1 \mathrm{~Sv}=100 \mathrm{rem}$.

$1 \mathrm{Ci}=3.7 \times 10^{10} \mathrm{~Bq}$. 
Table 4.1-4. Dose Coefficients for Inhalation and Ingestion of Radionuclides of Interest Based on ICRP 72 Dosimetric Methods

\begin{tabular}{|c|c|c|c|}
\hline \multirow[b]{2}{*}{ Primary Radionuclide } & \multirow{2}{*}{$\begin{array}{c}\text { Short-lived Decay } \\
\text { Product }\end{array}$} & \multicolumn{2}{|c|}{ Dose Coefficient (Sv/Bq) ${ }^{a}$} \\
\hline & & Inhalation & Ingestion \\
\hline \multirow[t]{2}{*}{ Strontium-90 } & & 1.6E-07 & 2.8E-08 \\
\hline & Yttrium-90 & $1.5 \mathrm{E}-09$ & $2.7 \mathrm{E}-09$ \\
\hline Technetium-99 & & $1.3 \mathrm{E}-08$ & $6.4 \mathrm{E}-10$ \\
\hline \multirow[t]{3}{*}{ Tin-126 } & & $2.8 \mathrm{E}-08$ & 4.7E-09 \\
\hline & Antimony-126m & $2.0 \mathrm{E}-11$ & 3.6E-11 \\
\hline & Antimony-126 & 3.2E-09 & 2.4E-09 \\
\hline \multirow[t]{2}{*}{ Cesium-137 } & & $3.9 \mathrm{E}-08$ & 1.3E-08 \\
\hline & Barium-137m & - & - \\
\hline \multicolumn{4}{|c|}{ Thorium Series (4n) } \\
\hline Plutonium-240 & & 1.2E-04 & $2.5 \mathrm{E}-07$ \\
\hline Uranium-236 & & 8.7E-06 & 4.7E-08 \\
\hline Thorium-232 & & 1.1E-04 & 2.3E-07 \\
\hline \multirow[t]{2}{*}{ Radium-228 } & & $1.6 \mathrm{E}-05$ & $6.9 \mathrm{E}-07$ \\
\hline & Actinium-228 & $2.5 \mathrm{E}-08$ & $4.3 \mathrm{E}-10$ \\
\hline Uranium-232 & & 3.7E-05 & $3.3 \mathrm{E}-07$ \\
\hline \multirow[t]{8}{*}{ Thorium-228 } & & $4.0 \mathrm{E}-05$ & $7.2 \mathrm{E}-08$ \\
\hline & Radium-224 & $3.4 \mathrm{E}-06$ & $6.5 \mathrm{E}-08$ \\
\hline & Radon-220 & - & - \\
\hline & Polonium-216 & - & - \\
\hline & Lead-212 & $1.9 \mathrm{E}-07$ & 6.0E-09 \\
\hline & Bismuth-212 & $3.1 \mathrm{E}-08$ & $2.6 \mathrm{E}-10$ \\
\hline & Polonium-212 & - & - \\
\hline & Thallium-208 & - & - \\
\hline \multicolumn{4}{|c|}{ Neptunium Series $(4 n+1)$} \\
\hline Americium-241 & & 9.6E-05 & 2.0E-07 \\
\hline \multirow[t]{2}{*}{ Neptunium-237 } & & $5.0 \mathrm{E}-05$ & 1.1E-07 \\
\hline & Protactinium-233 & $3.9 \mathrm{E}-09$ & $8.7 \mathrm{E}-10$ \\
\hline Uranium-233 & & $9.6 \mathrm{E}-06$ & 5.1E-08 \\
\hline \multirow[t]{9}{*}{ Thorium-229 } & & $2.4 \mathrm{E}-04$ & $4.9 \mathrm{E}-07$ \\
\hline & Radium-225 & 7.7E-06 & $9.9 \mathrm{E}-08$ \\
\hline & Actinium-225 & $8.5 \mathrm{E}-06$ & $2.4 \mathrm{E}-08$ \\
\hline & Francium-221 & - & - \\
\hline & Astatine-217 & - & - \\
\hline & Bismuth-213 & $3.0 \mathrm{E}-08$ & $2.0 \mathrm{E}-10$ \\
\hline & Polonium-213 & - & - \\
\hline & Thallium-209 & - & - \\
\hline & Lead-209 & $6.1 \mathrm{E}-11$ & 5.7E-11 \\
\hline
\end{tabular}


Table 4.1-4. Dose Coefficients for Inhalation and Ingestion of Radionuclides of Interest Based on ICRP 72 Dosimetric Methods (Continued)

\begin{tabular}{|c|c|c|c|}
\hline \multirow[b]{2}{*}{ Primary Radionuclide } & \multirow{2}{*}{$\begin{array}{c}\text { Short-lived Decay } \\
\text { Product }\end{array}$} & \multicolumn{2}{|c|}{ Dose Coefficient (Sv/Bq) ${ }^{a}$} \\
\hline & & Inhalation & Ingestion \\
\hline \multicolumn{4}{|c|}{ Uranium Series $(4 n+2)$} \\
\hline Plutonium-242 & & $1.1 \mathrm{E}-04$ & $2.4 \mathrm{E}-07$ \\
\hline \multirow[t]{4}{*}{ Uranium-238 } & & $8.0 E-06$ & 4.5E-08 \\
\hline & Thorium-234 & 7.7E-09 & 3.4E-09 \\
\hline & Protactinium-234m & - & - \\
\hline & Protactinium-234 & $4.0 \mathrm{E}-10$ & $5.1 \mathrm{E}-10$ \\
\hline Plutonium-238 & & 1.1E-04 & 2.3E-07 \\
\hline Uranium-234 & & $9.4 \mathrm{E}-06$ & $4.9 \mathrm{E}-08$ \\
\hline Thorium-230 & & $1.0 \mathrm{E}-04$ & $2.1 \mathrm{E}-07$ \\
\hline \multirow[t]{8}{*}{ Radium-226 } & & $9.5 \mathrm{E}-06$ & 2.8E-07 \\
\hline & Radon-222 & - & - \\
\hline & Polonium-218 & - & - \\
\hline & Lead-214 & $1.5 \mathrm{E}-08$ & 1.4E-10 \\
\hline & Astatine-218 & - & $-{ }^{a}$ \\
\hline & Bismuth-214 & $1.4 \mathrm{E}-08$ & 1.1E-10 \\
\hline & Polonium-214 & - & - \\
\hline & Thallium-210 & - & - \\
\hline \multirow[t]{3}{*}{ Lead-210 } & & $5.6 \mathrm{E}-06$ & $6.9 \mathrm{E}-07$ \\
\hline & Bismuth-210 & $9.3 \mathrm{E}-08$ & $1.3 \mathrm{E}-09$ \\
\hline & Polonium-210 & 4.3E-06 & $1.2 \mathrm{E}-06$ \\
\hline \multicolumn{4}{|c|}{ Actinium Series $(4 n+3)$} \\
\hline \multirow[t]{2}{*}{ Americium-243 } & & $9.6 \mathrm{E}-05$ & $2.0 \mathrm{E}-07$ \\
\hline & Neptunium-239 & 1.0E-09 & $8.0 \mathrm{E}-10$ \\
\hline Plutonium-239 & & $1.2 \mathrm{E}-04$ & 2.5E-07 \\
\hline \multirow[t]{2}{*}{ Uranium-235 } & & $8.5 \mathrm{E}-06$ & 4.7E-08 \\
\hline & Thorium-231 & $3.3 \mathrm{E}-10$ & $3.4 \mathrm{E}-10$ \\
\hline Protactinium-231 & & $1.4 \mathrm{E}-04$ & 7.1E-07 \\
\hline \multirow[t]{10}{*}{ Actinium-227 } & & $5.5 \mathrm{E}-04$ & 1.1E-06 \\
\hline & Thorium-227 & $1.0 \mathrm{E}-05$ & $8.8 \mathrm{E}-09$ \\
\hline & Francium-223 & $8.9 \mathrm{E}-10$ & $2.4 \mathrm{E}-09$ \\
\hline & Radium-223 & 8.7E-06 & $1.0 \mathrm{E}-07$ \\
\hline & Radon-219 & - & - \\
\hline & Polonium-215 & - & - \\
\hline & Lead-211 & $1.2 \mathrm{E}-08$ & $1.8 \mathrm{E}-10$ \\
\hline & Bismuth-211 & - & - \\
\hline & Thallium-207 & - & - \\
\hline & Polonium-211 & - & - \\
\hline
\end{tabular}

Source: MO0503SPADCESR.000 [DIRS 172896]

Notes: $\quad{ }^{\mathrm{a}}$ The DTN uses the term Dose Conversion Factors instead of Dose Coefficients

$1 \mathrm{~Sv}=100 \mathrm{rem}$

$1 \mathrm{Ci}=3.7 \times 10^{10} \mathrm{~Bq}$ 
Table 4.1-5. Dose Coefficients for Exposure to Contaminated Soil Surface for Radionuclides of Interest

\begin{tabular}{|c|c|c|c|}
\hline \multirow[b]{2}{*}{ Primary Radionuclide } & \multirow{2}{*}{$\begin{array}{c}\text { Short-lived Decay } \\
\text { Product }\end{array}$} & \multicolumn{2}{|c|}{ Dose Coefficient (Sv/s per Bq/m ${ }^{2}$ ) } \\
\hline & & Consistent with ICRP $30^{\circ}$ & Consistent with ICRP 72 $^{\mathrm{b}}$ \\
\hline \multirow[t]{2}{*}{ Strontium-90 } & & 2.84E-19 & 1.64E-18 \\
\hline & Yttrium-90 & $5.32 \mathrm{E}-18$ & $1.10 \mathrm{E}-16$ \\
\hline Technetium-99 & & $7.80 \mathrm{E}-20$ & $6.47 \mathrm{E}-20$ \\
\hline \multirow[t]{3}{*}{ Tin-126 } & & $5.47 \mathrm{E}-17$ & $4.82 \mathrm{E}-17$ \\
\hline & Antimony-126m & $1.52 \mathrm{E}-15$ & $1.55 \mathrm{E}-15$ \\
\hline & Antimony-126 & $2.78 \mathrm{E}-15$ & $2.72 \mathrm{E}-15$ \\
\hline \multirow[t]{2}{*}{ Cesium-137 } & & $2.85 \mathrm{E}-19$ & $2.99 \mathrm{E}-18$ \\
\hline & Barium-137m & $5.86 \mathrm{E}-16$ & $5.79 \mathrm{E}-16$ \\
\hline \multicolumn{4}{|c|}{ Thorium Series (4n) } \\
\hline Plutonium-240 & & 8.03E-19 & $6.01 \mathrm{E}-19$ \\
\hline Uranium-236 & & $6.50 \mathrm{E}-19$ & 5.03E-19 \\
\hline Thorium-232 & & $5.51 \mathrm{E}-19$ & 4.55E-19 \\
\hline \multirow[t]{2}{*}{ Radium-228 } & & $0.00 \mathrm{E}+00$ & $0.00 \mathrm{E}+00$ \\
\hline & Actinium-228 & $9.28 \mathrm{E}-16$ & $9.39 E-16$ \\
\hline Uranium-232 & & $1.01 E-18$ & 8.07E-19 \\
\hline \multirow[t]{8}{*}{ Thorium-228 } & & $2.35 \mathrm{E}-18$ & 2.13E-18 \\
\hline & Radium-224 & 9.57E-18 & $9.15 \mathrm{E}-18$ \\
\hline & Radon-220 & $3.81 \mathrm{E}-19$ & 3.69E-19 \\
\hline & Polonium-216 & $1.65 \mathrm{E}-20$ & 1.61E-20 \\
\hline & Lead-212 & 1.43E-16 & $1.35 \mathrm{E}-16$ \\
\hline & Bismuth-212 & $1.79 E-16$ & 2.25E-16 \\
\hline & Polonium-212 & $0.00 \mathrm{E}+00$ & $0.00 \mathrm{E}+00$ \\
\hline & Thallium-208 & $2.98 \mathrm{E}-15$ & 2.97E-15 \\
\hline \multicolumn{4}{|c|}{ Neptunium Series $(4 n+1)$} \\
\hline Americium-241 & & $2.75 \mathrm{E}-17$ & $2.33 \mathrm{E}-17$ \\
\hline \multirow[t]{2}{*}{ Neptunium-237 } & & $2.87 \mathrm{E}-17$ & $2.52 \mathrm{E}-17$ \\
\hline & Protactinium-233 & $1.95 \mathrm{E}-16$ & $1.86 \mathrm{E}-16$ \\
\hline Uranium-233 & & 7.16E-19 & $5.99 E-19$ \\
\hline \multirow[t]{9}{*}{ Thorium-229 } & & 8.54E-17 & 7.89E-17 \\
\hline & Radium-225 & $1.33 E-17$ & 1.07E-17 \\
\hline & Actinium-225 & $1.58 \mathrm{E}-17$ & 1.47E-17 \\
\hline & Francium-221 & $2.98 \mathrm{E}-17$ & 2.84E-17 \\
\hline & Astatine-217 & 3.03E-19 & 2.93E-19 \\
\hline & Bismuth-213 & $1.32 \mathrm{E}-16$ & $1.68 \mathrm{E}-16$ \\
\hline & Polonium-213 & $0.00 \mathrm{E}+00$ & $0.00 \mathrm{E}+00$ \\
\hline & Thallium-209 & $1.90 \mathrm{E}-15$ & $1.92 \mathrm{E}-15$ \\
\hline & Lead-209 & 3.01E-19 & 3.19E-18 \\
\hline
\end{tabular}


Table 4.1-5. Dose Coefficients for Exposure to Contaminated Soil Surface for Radionuclides of | Interest (Continued)

\begin{tabular}{|c|c|c|c|}
\hline \multirow[b]{2}{*}{ Primary Radionuclide } & \multirow{2}{*}{$\begin{array}{c}\text { Short-lived Decay } \\
\text { Product }\end{array}$} & \multicolumn{2}{|c|}{ Dose Coefficient (Sv/s per $\mathrm{Bq} / \mathrm{m}^{2}$ ) } \\
\hline & & Consistent with ICRP $30^{\mathrm{a}}$ & Consistent with ICRP $72^{b}$ \\
\hline \multicolumn{4}{|c|}{ Uranium Series $(4 n+2)$} \\
\hline Plutonium-242 & & 6.67E-19 & 4.98E-19 \\
\hline \multirow[t]{4}{*}{ Uranium-238 } & & $5.51 \mathrm{E}-19$ & 4.23E-19 \\
\hline & Thorium-234 & 8.32E-18 & $7.49 \mathrm{E}-18$ \\
\hline & Protactinium-234m & $1.53 \mathrm{E}-17$ & $1.08 \mathrm{E}-16$ \\
\hline & Protactinium-234 & $1.84 \mathrm{E}-15$ & $1.80 \mathrm{E}-15$ \\
\hline Plutonium-238 & & 8.38E-19 & $6.26 \mathrm{E}-19$ \\
\hline Uranium-234 & & $7.48 \mathrm{E}-19$ & $5.86 \mathrm{E}-19$ \\
\hline Thorium-230 & & $7.50 \mathrm{E}-19$ & $6.37 \mathrm{E}-19$ \\
\hline \multirow[t]{8}{*}{ Radium-226 } & & $6.44 \mathrm{E}-18$ & $6.11 \mathrm{E}-18$ \\
\hline & Radon-222 & $3.95 \mathrm{E}-19$ & $3.82 \mathrm{E}-19$ \\
\hline & Polonium-218 & 8.88E-21 & $8.66 \mathrm{E}-21$ \\
\hline & Lead-214 & $2.44 E-16$ & $2.40 \mathrm{E}-16$ \\
\hline & Astatine-218 & 4.18E-18 & $3.64 \mathrm{E}-18$ \\
\hline & Bismuth-214 & $1.41 \mathrm{E}-15$ & $1.44 \mathrm{E}-15$ \\
\hline & Polonium-214 & 8.13E-20 & 7.93E-20 \\
\hline & Thallium-210 & & $-^{c}$ \\
\hline \multirow[t]{3}{*}{ Lead-210 } & & $2.48 \mathrm{E}-18$ & 2.13E-18 \\
\hline & Bismuth-210 & $1.05 E-18$ & $3.51 \mathrm{E}-17$ \\
\hline & Polonium-210 & 8.29E-21 & 8.09E-21 \\
\hline \multicolumn{4}{|c|}{ Actinium Series $(4 n+3)$} \\
\hline \multirow[t]{2}{*}{ Americium-243 } & & 5.35E-17 & 4.79E-17 \\
\hline & Neptunium-239 & 1.63E-16 & $1.54 \mathrm{E}-16$ \\
\hline Plutonium-239 & & 3.67E-19 & $2.84 \mathrm{E}-19$ \\
\hline \multirow[t]{2}{*}{ Uranium-235 } & & $1.48 \mathrm{E}-16$ & $1.40 \mathrm{E}-16$ \\
\hline & Thorium-231 & $1.85 \mathrm{E}-17$ & $1.55 \mathrm{E}-17$ \\
\hline Protactinium-231 & & 4.07E-17 & $3.78 \mathrm{E}-17$ \\
\hline \multirow[t]{10}{*}{ Actinium-227 } & . & $1.57 \mathrm{E}-19$ & $1.41 \mathrm{E}-19$ \\
\hline & Thorium-227 & $1.04 \mathrm{E}-16$ & $9.81 \mathrm{E}-17$ \\
\hline & Francium-223 & $5.65 \mathrm{E}-17$ & 7.76E-17 \\
\hline & Radium-223 & $1.28 \mathrm{E}-16$ & $1.21 \mathrm{E}-16$ \\
\hline & Radon-219 & $5.49 \mathrm{E}-17$ & $5.28 \mathrm{E}-17$ \\
\hline & Polonium-215 & $1.74 \mathrm{E}-19$ & $1.68 \mathrm{E}-19$ \\
\hline & Lead-211 & $5.08 \mathrm{E}-17$ & $9.50 \mathrm{E}-17$ \\
\hline & Bismuth-211 & $4.58 \mathrm{E}-17$ & $4.40 \mathrm{E}-17$ \\
\hline & Thallium-207 & $3.76 \mathrm{E}-18$ & $5.56 \mathrm{E}-17$ \\
\hline & Polonium-211 & $7.61 \mathrm{E}-18$ & $7.42 \mathrm{E}-18$ \\
\hline \multicolumn{4}{|c|}{ Sources: a MO0407SPACRBSM.002 [DIRS 170677] } \\
\hline \multicolumn{4}{|l|}{ Note: $\quad{ }^{\mathbf{c}}$ Not included } \\
\hline
\end{tabular}




\subsubsection{Direct Inputs Used for Calculation of Inhalation Dose Factors for Volcanic | Eruption}

Inhalation dose factors for volcanic eruption were calculated using the inhalation dose coefficients (Tables 4.1-3 and 4.1-4), time spent in receptor environments, and the corresponding breathing rates (Table 4.1-1). In addition, average values of the estimated proportion of population for the volcanic scenario were used from MO0407SPACRBSM.002 [DIRS 170677] and are listed in Table 4.1-6.

Table 4.1-6. Population Proportion Values Used for Calculating Inhalation Dose Factors

\begin{tabular}{|l|c|}
\hline \multicolumn{1}{|c|}{ Population group } & Average Population Proportion \\
\hline Non-workers & 0.392 \\
\hline Commuters & 0.125 \\
\hline Local outdoor workers & 0.055 \\
\hline Local indoor workers & 0.428 \\
\hline
\end{tabular}

The data described above were used in Section 6.3.

\subsection{CRITERIA}

Three requirements from the Project Requirements Document (Canori and Leitner 2003 [DIRS 166275], Table 2-3) are applicable to this analysis (Table 4.2-1).

Table 4.2-1. Requirements Applicable to this Analysis

\begin{tabular}{|c|l|c|}
\hline $\begin{array}{c}\text { Requirement } \\
\text { Number }\end{array}$ & \multicolumn{1}{|c|}{ Requirement Title } & $\begin{array}{c}\text { Related } \\
\text { Regulation }\end{array}$ \\
\hline PRD-002/T-015 & Requirements for Performance Assessment & $10 \mathrm{CFR} 63.114$ \\
\hline PRD-002/T-026 & Required Characteristics of the Reference Biosphere & $10 \mathrm{CFR} 63.305$ \\
\hline PRD-002/T-028 & Required Characteristics of the Reasonably Maximaliy Exposed Individual & $10 \mathrm{CFR} 63.312$ \\
\hline
\end{tabular}

Source: Canori and Leitner 2003 [DIRS 166275], Table 2-3.

In addition to the requirements listed in Table 4.2-1, definition of terms in 10 CFR 63.2 and description of concepts in 10 CFR 63.102 [DIRS 173164] that are relevant to biosphere modeling are also applicable to this analysis.

Listed below are NRC acceptance criteria from Sections 2.2.1.2.13 (Redistribution of Radionuclides in Soil) and 2.2.1.3.14 (Biosphere Characteristics) of the Yucca Mountain Review Plan, Final Report (NRC 2003 [DIRS 163274]), based on the requirements of 10 CFR 63.114, 63.305, and 63.312 [DIRS 173164], that were considered when modeling biosphere characteristics. 
Acceptance Criteria from Section 2.2.1.3.13, Redistribution of Radionuclides in Soil

Acceptance Criterion 1, System Description and Model Integration Are Adequate.

(1) Total system performance assessment adequately incorporates important features, physical phenomena and couplings between different models, and uses consistent and appropriate assumptions throughout the abstraction of redistribution of radionuclides in the soil abstraction process;

(2) The total system performance assessment model abstraction identifies and describes aspects of redistribution of radionuclides in soil that are important to repository performance, including the technical bases for these descriptions. For example, the abstraction should include modeling of the deposition of contaminated material in the soil and determination of the depth distribution of the deposited radionuclides;

(3) Relevant site features, events, and processes have been appropriately modeled in the abstraction of redistribution of radionuclides, from surface processes, and sufficient technical bases are provided; and

\section{Acceptance Criterion 2, Data Are Sufficient for Model Justification.}

(1) Behavioral, hydrological, and geochemical values used in the license application are adequately justified (e.g., irrigation and precipitation rates, erosion rates, radionuclide solubility values, etc.). Adequate descriptions of how the data were used, interpreted, and appropriately synthesized into the parameters are provided; and

(2) Sufficient data (e.g., field, laboratory, and natural analog data) are available to adequately define relevant parameters and conceptual models necessary for developing the abstraction of redistribution of radionuclides in soil in the total system performance assessment.

Acceptance Criterion 3, Data Uncertainty Is Characterized and Propagated Through the Model Abstraction.

(1) Models use parameter values, assumed ranges, probability distributions, and bounding assumptions that are technically defensible, reasonably account for uncertainties and variabilities, do not result in an under-representation of the risk estimate, and are consistent with the characteristics of the reasonably maximally exposed individual in 10 CFR Part 63;

(2) The technical bases for the parameter values and ranges in the total system performance assessment abstraction are consistent with data from the Yucca Mountain region, e.g., Amargosa Valley survey, studies of surface processes in the Fortymile Wash drainage basin, applicable laboratory testing, natural analogs, or other valid sources of data. For example, soil types, crop types, plow depths, and irrigation rates should be consistent with current farming practices, and data on the airborne particulate concentration should be based on the resuspension of appropriate material in a climate and level of disturbance 
similar to that which is expected to be found at the location of the reasonably maximally exposed individual, during the compliance time period;

(3) Uncertainty is adequately represented in parameters for conceptual models, process models, and alternative conceptual models considered in developing the total system performance assessment abstraction of redistribution of radionuclides in soil, either through sensitivity analyses, conservative limits, or bounding values supported by data, as necessary. Correlations between input values are appropriately established in the total system performance assessment.

\section{Acceptance Criteria from Section 2.2.1.3.14, Biosphere Characteristics}

\section{Acceptance Criterion 1, System Description and Model Integration Are Adequate.}

(3) Assumptions are consistent between the biosphere characteristics modeling and other abstractions. For example, the U.S. Department of Energy should ensure that the modeling of features, events, and processes, such as climate change, soil types, sorption coefficients, volcanic ash properties, and the physical and chemical properties of radionuclides are consistent with assumptions in other total system performance assessment abstractions.

\section{Acceptance Criterion 2, Data Are Sufficient for Model Justification.}

(1) The parameter values used in the license application are adequately justified (e.g., behaviors and characteristics of the residents of the Town of Amargosa Valley, Nevada, characteristics of the reference biosphere, etc.) and consistent with the definition of the reasonably maximally exposed individual in 10 CFR Part 63. Adequate descriptions of how the data were used, interpreted, and appropriately synthesized into the parameters are provided; and

(2) Data are sufficient to assess the degree to which features, events, and processes related to biosphere characteristics modeling have been characterized and incorporated in the abstraction. As specified in 10 CFR Part 63, the U.S. Department of Energy should ensure that the modeling of features, events, and processes, such as climate change, soil types, sorption coefficients, volcanic ash properties, and the physical and chemical properties of radionuclides are consistent with assumptions in other total system performance assessment abstractions.

\section{Acceptance Criterion 3, Data Uncertainty Is Characterized and Propagated Through the Model Abstraction.}

(1) Models use parameter values, assumed ranges, probability distributions, and bounding assumptions that are technically defensible, reasonably account for uncertainties and variabilities, do not result in an under-representation of the risk estimate, and are consistent with the definition of the reasonably maximally exposed individual in $10 \mathrm{CFR}$ Part 63; 
(2) The technical bases for the parameter values and ranges in the abstraction, such as consumption rates, plant and animal uptake factors, mass-loading factors, and biosphere dose conversion factors, are consistent with site characterization data, and are technically defensible.

\subsection{CODES, STANDARDS, AND REGULATIONS}

No codes, standards, or regulations, other than those identified in the Project Requirements Document (Canori and Leitner 2003 [DIRS 166275], Table 2-3) and determined to be applicable, were used in this analysis. 


\section{ASSUMPTIONS}

No assumptions were used in this analysis. 
INTENTIONALLY LEFT BLANK 


\section{SCIENTIFIC ANALYSIS DISCUSSION}

The objectives of this analysis were to calculate:

1. The BDCFs for the volcanic ash exposure scenario - these BDCFs are used as input to the TSPA model and allow calculating the expected annual dose to the reasonably maximally exposed individual (RMEI) from a given concentration of radionuclides in surface soil and thickness of volcanic ash deposited on the ground. The dose to the RMEI is used to evaluate compliance with the individual protection standard (10 CFR 63.311 [DIRS 173164]).

2. The inhalation dose factors that can be used by TSPA to calculate the expected inhalation dose accrued during the period of deposition of volcanic ash on the ground during the volcanic eruption.

The above quantities are calculated using two dosimetric approaches: the ICRP 30 and ICRP 72. The first approach is based on the concepts recommended in ICRP Publication 26 (ICRP 1977 [DIRS 101075]) and the dosimetric methods for intakes of radionuclides by workers outlined in ICRP Publication 30 (ICRP 1979 [DIRS 110386]; ICRP 1980 [DIRS 110351]; ICRP 1981 [DIRS 110352]). This approach is consistent with the individual protection standard defined in terms of total effective dose equivalent.

The ICRP 72 approach is based on the concepts recommended in ICRP Publication 60 (ICRP 1991 [DIRS 101836]). ICRP Publication 60 introduced a new dosimetric quantity, the effective dose, which uses an expanded list of tissues and organs and revised values of tissue and organ weighting factors. In addition, biokinetic and dosimetric models were updated to include new information that became available since ICRP Publication 30. A set of age-dependent dose coefficients for intakes of radionuclides by members of the public was developed and documented in a series of reports compiled in ICRP Publication 72 (ICRP 1996 [DIRS 152446]). ICRP Publication 72 is presently the most current compilation of dose coefficients for internal intakes and the approach consistent with the ICRP Publication 72 dosimetric methods is used in this analysis to develop biosphere input to TSPA. The ICRP 30-based values are presented in this report for comparison purposes.

The scenario classes considered for TSPA include the nominal scenario, seismic scenario, and igneous scenario classes (BSC 2005 [DIRS 173309], Section 2.2.2). Biosphere exposure scenarios should not be confused with TSPA scenario classes. The biosphere exposure scenarios consider radiological consequences of radionuclide releases to the reference biosphere in a given medium, such as the groundwater or volcanic ash, irrespective of the cause of contamination in that medium. These two release modes match the two biosphere exposure scenarios and the associated biosphere models developed to support the TSPA analyses and described in the Biosphere Model Report (BSC 2004 [DIRS 169460]). The biosphere exposure scenarios consider radiological consequences of radionuclide releases to the reference biosphere in groundwater or volcanic tephra.

For the volcanic ash scenario, the initial source of radionuclides in the biosphere for the volcanic ash exposure scenario is contaminated volcanic ash deposited on the ground surface as the result of a volcanic eruption. After radionuclides enter the biosphere, radionuclide migration through 
the biosphere occurs due to a number of transport processes that lead to contamination and accumulation in the environmental media (e.g., soil, air, flora, and fauna). Human exposure to radionuclides in the environment arises when people come in contact with contaminated environmental media.

BDCFs for the volcanic ash exposure scenario apply to the volcanic eruption modeling case of the igneous scenario class. A detailed description of the volcanic ash exposure scenario, including the associated conceptual and mathematical models, is presented in the Biosphere Model Report (BSC 2004 [DIRS 169460], Sections 6.3.2 and 6.5).

\subsection{GENERAL CONSIDERATIONS}

\subsubsection{Radionuclides Included in the Analysis}

The radionuclides of interest for the biosphere model depend on the exposure scenario, as discussed in the Biosphere Model Report (BSC 2004 [DIRS 169460], Section 6.1.3). The following 23 radionuclides were identified as important for the TSPA scenario classes involving radionuclide release during a volcanic eruption: strontium-90 $\left({ }^{90} \mathrm{Sr}\right)$, technetium-99 $\left({ }^{99} \mathrm{Tc}\right)$, tin-126 $\left({ }^{126} \mathrm{Sn}\right)$, cesium-137 $\left({ }^{137} \mathrm{Cs}\right)$, lead-210 $\left({ }^{210} \mathrm{~Pb}\right)$, radium-226 $\left({ }^{226} \mathrm{Ra}\right)$, actinium-227 $\left({ }^{227} \mathrm{Ac}\right)$, thorium-229 $\left.{ }^{229} \mathrm{Th}\right)$, thorium-230 $\left({ }^{230} \mathrm{Th}\right)$, thorium-232 $\left({ }^{232} \mathrm{Th}\right)$, protactinium-231 $\left({ }^{231} \mathrm{~Pa}\right)$, uranium-232 $\left({ }^{232} \mathrm{U}\right)$, uranium-233 $\left({ }^{233} \mathrm{U}\right)$, uranium-234 $\left({ }^{234} \mathrm{U}\right)$, uranium-236 $\left({ }^{236} \mathrm{U}\right)$, uranium-238 $\left({ }^{238} \mathrm{U}\right)$, neptunium-237 $\left({ }^{237} \mathrm{~Np}\right)$, plutonium-238 $\left({ }^{238} \mathrm{Pu}\right)$, plutonium-239 $\left({ }^{239} \mathrm{Pu}\right)$, plutonium-240 $\left({ }^{240} \mathrm{Pu}\right)$, plutonium-242 $\left({ }^{242} \mathrm{Pu}\right)$, americium-241 $\left({ }^{241} \mathrm{Am}\right)$, and americium-243 $\left({ }^{243} \mathrm{Am}\right)(\mathrm{BSC} 2004$ [DIRS 169460], Section 6.1.3). These radionuclides are referred to in this analysis as the primary radionuclides. The list includes radionuclides that are of potential importance during both the first 20,000 years and the period of up to 1,000,000 years (BSC 2004 [DIRS 169460], Section 6.1.3).

The biosphere model accounts for the decay products of the primary radionuclides. The short-lived decay products (half-lives less than 180 days) are considered to be in secular equilibrium with the parent radionuclide and their contributions to the BDCFs are included in the BDCF for the long-lived radionuclide (either a primary radionuclide or its long-lived decay product). Two decay products of the primary radionuclides, ${ }^{228} \mathrm{Th}$ and ${ }^{228} \mathrm{Ra}$, have half-lives greater than 180 days and are not automatically included in the BDCFs of the parent when the biosphere model is executed. Instead, for biosphere modeling they are treated like primary radionuclides. After their BDCFs are calculated, they are added to the BDCF of the parent primary radionuclide. In the case of ${ }^{232} \mathrm{Th}$, its BDCF includes the contribution from ${ }^{228} \mathrm{Ra},{ }^{228} \mathrm{Th}$, and their short-lived decay products. The BDCF for ${ }^{232} \mathrm{U}$ includes the contribution from ${ }^{228} \mathrm{Th}$ and its short-lived decay products.

\subsubsection{Description of the Volcanic Ash Exposure Scenario}

A detailed description of the volcanic ash exposure scenario, including the associated conceptual and mathematical models, is presented in the Biosphere Model Report (BSC 2004 [DIRS 169460], Sections 6.3.2 and 6.5). A brief summary of the main concepts and the modeling approach for the volcanic ash exposure scenario is presented in this section. 
The general scenario (the release of radionuclides to the reference biosphere, environmental transport, and the subsequent exposure of the RMEI) is shown schematically in Figure 6.1-1. The immediate source of radionuclides in the biosphere for this scenario is contaminated volcanic ash initially deposited on the ground surface following a volcanic eruption.

After radionuclides enter the biosphere, radionuclide migration through the biosphere occurs due to a number of transport processes that lead to contamination and accumulation in the environmental media (e.g., soil, air, flora, and fauna). The following environmental transport processes are explicitly included in the biosphere model for the volcanic ash exposure scenario:

- Resuspension of contaminated soil and ash

- Dry deposition of radionuclides on crop surfaces (resuspension of contaminated soil and subsequent adhesion of soil particles on crop surfaces)

- Translocation of contaminants from the site of deposition to the edible portions of crops

- Post-deposition contaminant retention by crops (including weathering processes)

- Radionuclide uptake by crops through the roots

- Radionuclide uptake by animals through consumption of contaminated feed and soil, and subsequent transfer to animal products

- Exhalation of radon from the soil.

These processes can be mapped to the biosphere-related FEPs listed in Table 1-1.

Human exposure to radionuclides in the environment arises when people come in direct (inhalation and ingestion) or indirect (external exposure) contact with contaminated environmental media. Table 6.1-1 provides a summary of human exposure pathways included in the biosphere model, as well as environmental media and typical activities that may cause radiation exposure. Inhalation exposure arising from gaseous emissions from a volcano was not considered because gaseous radionuclides were not included among radionuclides of interest (BSC 2004 [DIRS 169460], Section 6.1.3). 


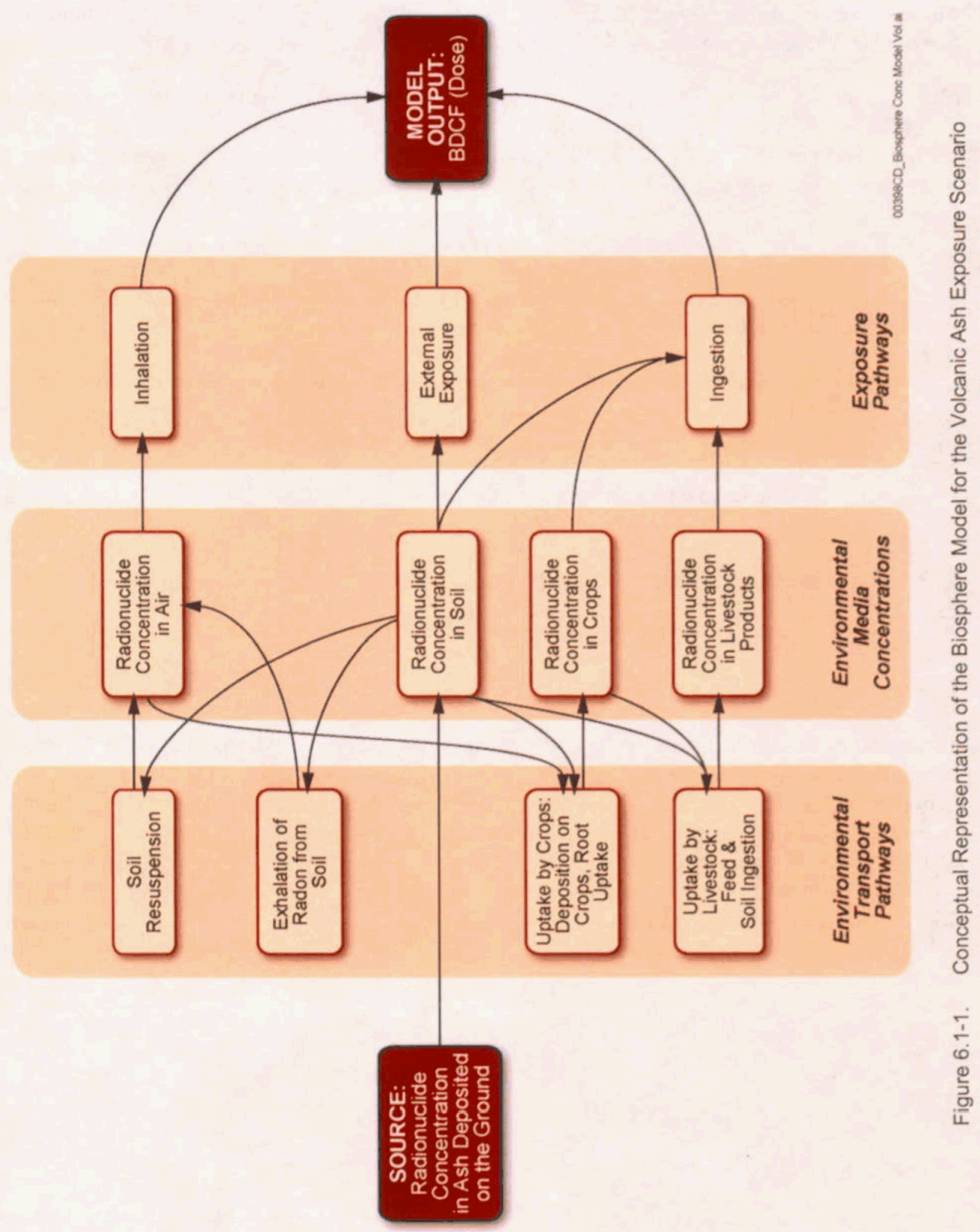


Table 6.1-1. RMEI Exposure Pathways for the Volcanic Ash Exposure Scenario

\begin{tabular}{|c|c|c|c|}
\hline $\begin{array}{l}\text { Environmental } \\
\text { Medium }\end{array}$ & $\begin{array}{l}\text { Exposure } \\
\text { Mode }\end{array}$ & Exposure Pathways & Examples of Typical Activities \\
\hline Soil & Ingestion & Inadvertent soil ingestion. & $\begin{array}{l}\text { Recreational activities, occupational } \\
\text { activities, gardening, and } \\
\text { consumption of fresh fruit and } \\
\text { vegetables. }\end{array}$ \\
\hline Soil & External & External radiation exposure. & $\begin{array}{l}\text { Activities on or near contaminated } \\
\text { soils. }\end{array}$ \\
\hline Air & Inhalation & $\begin{array}{l}\text { Breathing resuspended particles, and } \\
\text { gases }\left({ }^{222} \mathrm{Rn} \text { and progeny) }\right.\end{array}$ & $\begin{array}{l}\text { Outdoor activities, including soil- } \\
\text { disturbing activities related to work } \\
\text { and recreation. Domestic activities, } \\
\text { including sleeping. }\end{array}$ \\
\hline Plants & Ingestion & $\begin{array}{l}\text { Consumption of locally produced crops: } \\
\text { leafy vegetables, other vegetables, fruit, } \\
\text { and grain. }\end{array}$ & Eating contaminated crop foodstuffs. \\
\hline Animals & Ingestion & $\begin{array}{l}\text { Consumption of locally produced animal } \\
\text { products: meat, poultry, milk, and eggs. }\end{array}$ & $\begin{array}{l}\text { Eating contaminated animal product } \\
\text { foodstuffs. }\end{array}$ \\
\hline
\end{tabular}

Source: BSC 2004 [DIRS 169460], Table 6.3-3.

\subsubsection{Consideration of Climate Change}

Climate refers to the meteorological conditions that characteristically prevail in a particular region. The climate analysis for the Yucca Mountain region was performed using paleoclimate and paleoenvironmental reconstructions based on microfossil records from cores from Owens Lake, California and isotope data from samples of calcite deposits from Devils Hole, Death Valley National Park, Nevada. The sequence and duration of past climate periods are identified from the records and applied to the Yucca Mountain site, which has a similar climate setting. The temperature and precipitation records of present-day meteorological stations at colder and wetter sites are selected to represent future climate states (BSC 2004 [DIRS 170002], Section 6.6.1).

For modeling of climate change in TSPA, the climate shifts in a series of step changes between three different climate states in the first 10,000 years: present-day climate, monsoon climate (about twice the precipitation of the present day climate), and glacial transition climate (colder than monsoon but similar precipitation) (BSC 2003 [DIRS 166296], p. 79). Within the TSPA model, these shifts require coordinating the coupled submodels because they must all simultaneously change to the appropriate climate state. To support the modeling of climate change in TSPA, the BDCFs must also be appropriate for the given climates. The discussion of the effect of climate change on volcanic ash exposure scenario BDCFs is presented in Section 6.2.5.

\subsubsection{Definition of the Receptor}

The regulations for licensing the repository include an individual protection standard for the performance of the repository. This standard is expressed as the annual dose limit to a hypothetical person called the reasonably maximally exposed individual (RMEI) (10 CFR 63.311 [DIRS 173164]). Analysis of annual dose includes potentially pathways of I 
radionuclide transport and exposure (10 CFR 63.311 [DIRS 173164]). Changes in the reference biosphere, other than climate changes, are not included.

According to 10 CFR 63.312 [DIRS 173164], the RMEI is a hypothetical receptor that:

- Lives above the highest concentration of radionuclides in the plume of contamination

- Has a diet and lifestyle representative of people who now reside in the Amargosa Valley based on surveys of the people residing in the Amargosa Valley that determine current diets and lifestyles, and then use the mean values of these factors in the assessments conducted for 10 CFR 63.311 and 10 CFR 63.321

- Uses well water with average concentrations of radionuclides based on an annual water demand of 3,000 acre-feet

- Drinks 2 liters of water per day from wells drilled into the groundwater from a point above the highest concentration of radionuclides in the plume of contamination

- Is an adult who is metabolically and physiologically consistent with present knowledge of adults.

Of the above characteristics of the RMEI those that are related to contaminated water apply only to the groundwater exposure scenario and are not used in this analysis. The evaluation of RMEI's exposure from contaminated groundwater is provided in the Nominal Performance Biosphere Dose Conversion Factor Analysis (see Figure 1-1).

To satisfy 10 CFR 63.312(b) [DIRS 173164] criteria, the dietary and lifestyle characteristics of | the RMEI were determined based on surveys of the people living in the town of Amargosa Valley combined with national information on behavior patterns. Characteristics of the RMEI were developed in a separate analysis (BSC 2005 [DIRS 172827]).

\subsubsection{Biosphere Model}

This analysis was performed using a verified and validated model, ERMYN, which is described in the Biosphere Model Report (BSC 2004 [DIRS 169460]). The model files were obtained from the Model Warehouse (DTN: MO0306MWDBGSMF.001 [DIRS 163816]). These model files were created using GoldSim V.7.50.100. Since then, updated versions of GoldSim were baselined. Two of these versions: V.8.01 Service Pack 4 (BSC 2004 [DIRS 169695]) and V.8.02.300 (BSC 2004 [DIRS 172588]) were used in this analysis. Verification of the model files for GoldSim V.8.01 Service Pack 4 and V.8.02.300 is presented in Appendix C.

The ERMYN was developed to model the biosphere processes for radionuclides released from the geological repository at Yucca Mountain including the environmental transport of radionuclides and human exposure. The Biosphere Model Report (BSC 2004 [DIRS 169460]), which documents the ERMYN model: 
1. Describes the biosphere model objectives, the reference biosphere, human receptor, exposure scenarios, environmental transport pathways and human exposure pathways (BSC 2004 [DIRS 169460], Section 6.1)

2. Develops the biosphere conceptual model based on the site-specific FEPs, the reference biosphere and human receptor, and a number of assumptions (BSC 2004 [DIRS 169460], Sections 6.2 and 6.3)

3. Describes the biosphere mathematical model and its submodels based on the developed conceptual model and other published biosphere models (BSC 2004 [DIRS 169460], Sections 6.4 and 6.5)

4. Summarizes model input parameters and uncertainty distributions (BSC 2004 [DIRS 169460], Section 6.6)

5. Identifies model improvements compared with the previous biosphere model (BSC'2004 [DIRS 169460], Section 6.7)

6. Constructs the ERMYN implementation tool based on the biosphere mathematical model using GoldSim stochastic simulation software (BSC 2004 [DIRS 169460], Sections 6.8 and 6.9)

7. Verifies the ERMYN implementation tool in GoldSim (BSC 2004 [DIRS 169460], Section 6.10)

8. Validates the ERMYN model by comparing the conceptual and mathematical models and by comparing the numerical results with results from other published biosphere models (BSC 2004 [DIRS 169460], Section 7).

The ERMYN model was designed to perform an environmental radiation dose assessment and can calculate either radionuclide-specific dose or provide a radionuclide-specific BDCF for a given receptor. The use of the ERMYN model in the performance assessment is limited to calculation of BDCFs.

Input parameters for the biosphere model are developed and documented in a series of five model parameter reports:

- BSC 2004 [DIRS 169673]. Agricultural and Environmental Parameters for the Biosphere Model. ANL-MGR-MD-000006 REV 02.

- BSC 2005 [DIRS 172827]. Characteristics of the Receptor for the Biosphere Model. ANL-MGR-MD-000005 REV 04.

- BSC 2004 [DIRS 169672]. Environmental Transport Input Parameters for the Biosphere Model. ANL-MGR-MD-000007 REV 02.

- BSC 2004 [DIRS 169458]. Inhalation Exposure Input Parameters for the Biosphere Model. ANL-MGR-MD-000001 REV 03. 
- BSC 2004 [DIRS 169459]. Soil-Related Input Parameters for the Biosphere Model. ANL-NBS-MD-000009 REV 02.

The architecture of the biosphere model for the volcanic ash exposure scenario, including the submodels, is shown in Figure 6.1-2. The submodels address radionuclide transport to, and accumulation in, specific environmental media (e.g., soil, air, plants and animals) and the inhalation, ingestion, and external exposure pathways.

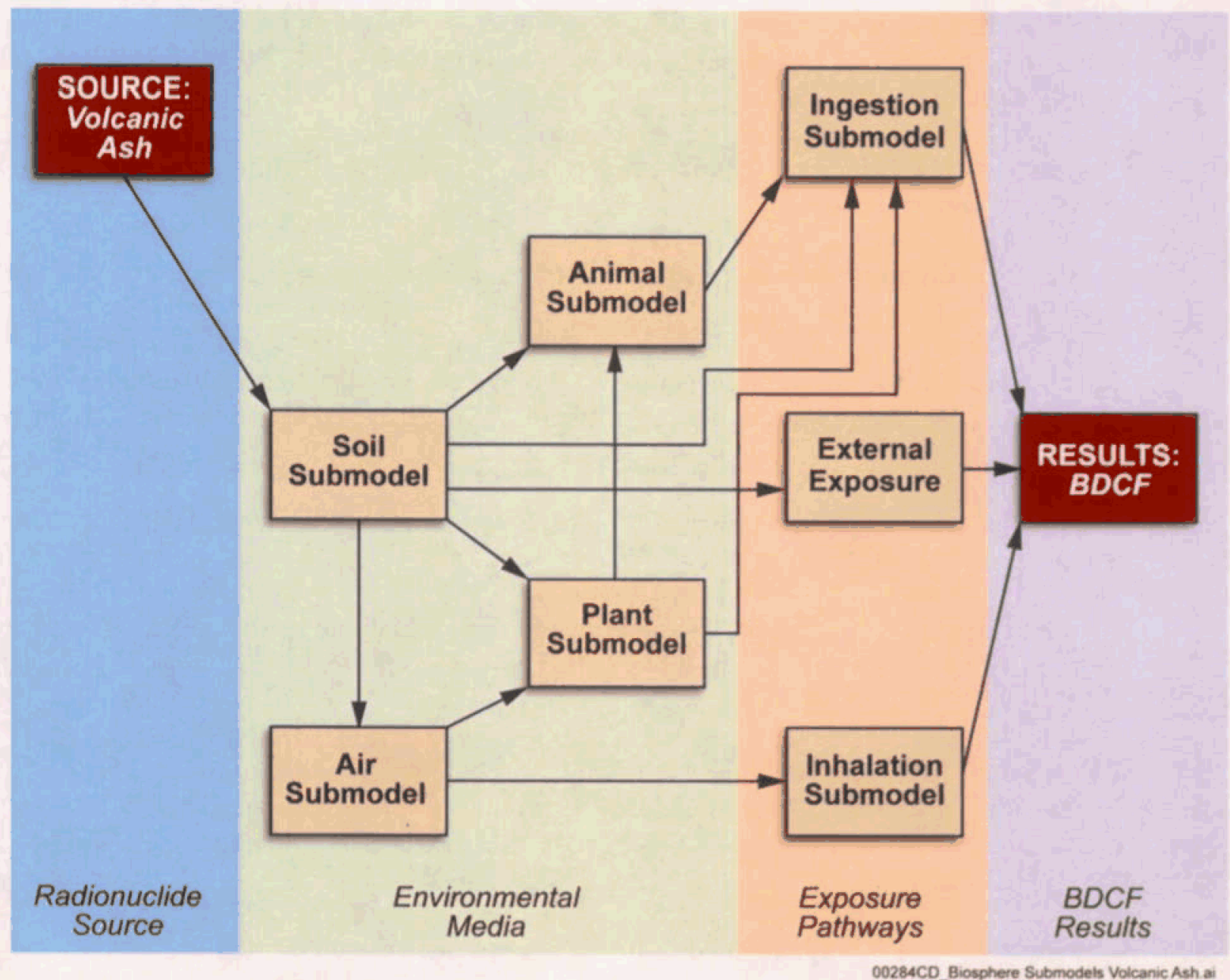

Figure 6.1-2. Relationship Between the Biosphere Submodels for the Volcanic Ash Exposure Scenario

\subsection{DEVELOPMENT OF BIOSPHERE DOSE CONVERSION FACTORS FOR THE VOLCANIC ASH EXPOSURE SCENARIO}

The BDCFs for the volcanic ash exposure scenario were calculated, using probabilistic analysis, in a series of simulations for each of the 23 primary radionuclides and two long-lived decay products, ${ }^{228} \mathrm{Th}$ and ${ }^{228} \mathrm{Ra}$ (see Section 6.1.1). Each simulation resulted in 1,000 model realizations. A model realization is one of the possible model outcomes obtained as a result of a single round of sampling of the model input parameters. This section describes the format and the summary of the results of biosphere modeling for the volcanic ash exposure scenario, as well as their use in the TSPA model. 


\subsubsection{Treatment of Uncertainty}

The probabilistic approach was chosen to develop BDCFs. This approach allows statistical sampling of parameter values defined by their probability distribution functions. This method, called Monte Carlo analysis, provides a quantitative evaluation of the parameter uncertainties and their impacts on the modeling outcome. The uncertainty in the model outcome is represented by the probability distribution functions of the BDCFs. The input parameter distributions were sampled using Latin Hypercube sampling in order to maintain consistency with the sampling technique used in TSPA (BSC 2005 [DIRS 173309], Table 2.10-2). When Latin Hypercube sampling is performed, the probability distribution is divided into intervals of equal probability. One sample is obtained at random from within each interval resulting in a more even and consistent set of sampling results when compared with conventional Monte Carlo sampling. For this analysis, the value of the random seed in GoldSim was set to 1 .

\subsubsection{Format of Biosphere Dose Conversion Factors for Volcanic Ash Exposure Scenario and Their Use in the Total System Performance Assessment Model}

The radionuclide concentration in the volcanic ash deposited on the ground surface is the only source of radionuclide contamination for the volcanic ash exposure scenario used for development of BDCFs in this analysis (direct inhalation of ash during eruption phase is treated separately - see Section 6.3). Environmental transport and RMEI exposure pathways discussed in the previous section depend linearly on this source.

The integration of the biosphere component into the TSPA model depends on the format of the source term, i.e., the activity concentration in the environmental medium that is the source of contamination in the biosphere. For the source of radionuclides expressed in terms of aerial activity concentration, BDCFs for the volcanic ash exposure scenario are a function of the thickness of contaminated soil-ash surface layer. Because of the anticipated gradual decrease in airborne particulate concentration over time, the BDCFs are also a function of time. For the volcanic ash exposure scenario, the BDCFs are given in the following format (BSC 2004 [DIRS 169460], Section 6.5.8.2):

$$
B D C F_{i}\left(d_{a}, t\right)=B D C F_{e x t, i n g, R n, i}+\left(B D C F_{i n h, v, i} f(t)+B D C F_{i n h, p, i}\right) g\left(d_{a}\right)
$$

where

$$
\begin{aligned}
& B D C F_{i}\left(d_{a}, t\right) \quad=\quad \mathrm{BDCF} \text { for primary radionuclide } i \text { for an ash depth } d_{a} \text { at time } t \\
& \text { following a volcanic eruption ( } \mathrm{Sv} / \mathrm{yr} \text { per } \mathrm{Bq} / \mathrm{m}^{2} \text { ) } \\
& B D C F_{\text {ext,ing,Rn,i }} \quad=\mathrm{BDCF} \text { for primary radionuclide } i \text { for external exposure, ingestion, } \\
& \text { and inhalation of radon decay products ( } \mathrm{Sv} / \mathrm{yr} \text { per } \mathrm{Bq} / \mathrm{m}^{2} \text { ) } \\
& B D C F_{i n h, v, i} \quad=\quad \mathrm{BDCF} \text { for primary radionuclide } i \text { for short-term inhalation at } \\
& \text { post-volcanic level of mass loading in excess of nominal mass } \\
& \text { loading ( } \mathrm{Sv} / \mathrm{yr} \text { per } \mathrm{Bq} / \mathrm{m}^{2} \text { ) } \\
& B D C F_{i n h, p, i}=\mathrm{BDCF} \text { for primary radionuclide } i \text { for long-term inhalation at } \\
& \text { nominal level of mass loading ( } \mathrm{Sv} / \mathrm{yr} \text { per } \mathrm{Bq} / \mathrm{m}^{2} \text { ) } \\
& f(t) \quad=\text { decay function describing reduction of mass loading with time } \\
& d_{a} \quad=\text { thickness of ash (m) }
\end{aligned}
$$


$g\left(d_{a}\right) \quad=$ function of ash thickness, $d_{a}$, representing the fraction of total activity that is available for resuspension.

The linearity of this equation is discussed in the Biosphere Model Report (BSC 2004 [DIRS 169460], Section 6.5.8.2).

Three BDCF components are calculated for each primary radionuclide. The first component, $B D C F_{\text {ext,ing, } R n, i}$, is time independent and accounts for three exposure pathways (exposure to sources external to the body, ingestion, and inhalation of radon decay products). The second and third BDCF components account for inhaling airborne particulates. Both of these components depend on ash thickness. The term $B D C F_{i n h, v, i}$ represents short-term inhalation exposure during the period of increased concentrations of resuspended particulates following volcanic eruption and is time-dependent because mass loading will gradually decrease after an eruption. The term $B D C F_{i n h, p, i}$ represents long-term inhalation of resuspended particulates under nominal conditions (i.e., when the mass loading is not elevated as the result of volcanic eruption).

The results of the BDCF calculations are in the format of 1,000 row vectors, one for each model realization, consisting of three BDCF components for each of the 23 primary radionuclides (i.e., $3 \times 23=69 \mathrm{BDCF}$ components per vector) and a value of critical thickness, as explained below. A vector can be regarded as a one-dimensional array containing the results of a single realization of the biosphere model for all primary radionuclides. Technically, the model is executed separately for individual primary radionuclides. The vectors are then produced by compiling the BDCFs for a given realization number. Such an approach is valid because for a given model realization number all radionuclide-independent parameters are the same regardless of a radionuclide.

The function of time, $f(t)$ in Equation 6.2-1, accounts for the reduction of mass loading in the years immediately following volcanic eruption. Mass loading decreases exponentially with time (modified from BSC 2004 [DIRS 169458], Equation 6.3-1) as

$$
f(t)=e^{-\lambda t}
$$

where

$\lambda \quad=$ mass loading decrease constant $(1 / \mathrm{yr})$

$t=$ time $(\mathrm{yr}) ; t=0$ is the first year after a volcanic eruption.

The mass loading decrease constant (Equation 6.2-2) depends on the ash thickness, and for an initial ash depth of less than $10 \mathrm{~mm}$, it is represented by a triangular probability distribution function with a mode of $0.33 / \mathrm{yr}$, a minimum of $0.2 / \mathrm{yr}$, and a maximum of $2.0 / \mathrm{yr}$ (DTN: MO0407SPAINEXI.002 [DIRS 170597]). For an initial ash depth of $10 \mathrm{~mm}$ or more, the mass loading decrease constant is represented by a triangular distribution with a mode of $0.20 / \mathrm{yr}$, a minimum of $0.125 / \mathrm{yr}$, and a maximum of 1.0/yr (DTN: MO0407SPAINEXI.002 [DIRS 170597]). 
The function of ash thickness (BSC 2004 [DIRS 169460], Section 6.5.1.2), $g\left(d_{a}\right)$, is expressed as

$$
g\left(d_{a}\right)= \begin{cases}1 & \text { when } d_{a}<d_{c} \\ \frac{d_{c}}{d_{a}} & \text { when } d_{a} \geq d_{c}\end{cases}
$$

where $d_{c}$ is the critical thickness of the ash layer. The critical thickness is defined as the thickness of the surface soil (ash) layer that is available for resuspension, and the purpose of the ash thickness function is to estimate the activity concentration in this layer for the source term given as an areal activity concentration of a radionuclide. Since this parameter is represented by a distribution, the value of the critical thickness corresponding to the specific BDCF row vector must be used. The critical thickness value is thus added to each of the 1,000 BDCF row vectors.

The calculations of the all-pathway dose for any given primary radionuclide are carried out in the TSPA model by combining the source term with the BDCFs. For the volcanic ash exposure scenario, the mass loading decrease function and the ash thickness must also be factored in. The total annual dose is the sum of the annual doses from individual radionuclides tracked by the TSPA (primary radionuclides), including their decay products. The total annual dose, computed by the TSPA model, is calculated as

$$
\begin{aligned}
D_{\text {total }}\left(d_{a}, t\right)= & \sum_{i} B D C F_{i}\left(d_{a} t\right) \times C s_{i}(t) \\
= & \sum_{i} B D C F_{e x t, i n g, R n, i} \times C s_{i}(t) \\
& +\sum_{i}\left(B D C F_{i n h, v, i} f(t)+B D C F_{i n h, p, i}\right) g\left(d_{a}\right) \times C s_{i}(t)
\end{aligned}
$$

where

$$
\begin{aligned}
& D_{\text {total }}\left(d_{a}, t\right) \quad=\quad \text { time-dependent total annual dose to a defined receptor resulting from } \\
& \text { the release of radionuclides from the repository including } \\
& \text { contributions from all radionuclides considered in the TSPA-LA } \\
& \text { (Sv/yr) } \\
& B D C F_{i}\left(d_{a}, t\right)=\text { BDCF for radionuclide } i \text { for an ash deposition depth of } d_{a} \text { at time } t \\
& \text { following a volcanic eruption ( } \mathrm{Sv} / \mathrm{yr} \text { per } \mathrm{Bq} / \mathrm{m}^{2} \text { ) } \\
& C s_{i}(t) \quad=\quad \text { time dependent activity concentration of radionuclide } i \text { in volcanic } \\
& \text { ash deposited on the ground }\left(\mathrm{Bq} / \mathrm{m}^{2}\right) \\
& B D C F_{\text {ext, ing, } R n, i}=\text { BDCF for radionuclide } i \text { for external exposure, ingestion and } \\
& \text { inhalation of radon decay products ( } \mathrm{Sv} / \mathrm{yr} \text { per } \mathrm{Bq} / \mathrm{m}^{2} \text { ) } \\
& B D C F_{i n h, v, i}=\text { BDCF for radionuclide } i \text { for inhalation of post-volcanic mass loading } \\
& \text { in addition to nominal mass loading following a volcanic eruption } \\
& \left(\mathrm{Sv} / \mathrm{yr} \text { per } \mathrm{Bq} / \mathrm{m}^{2}\right. \text { ) } \\
& B D C F_{\text {inh, }, i}=\mathrm{BDCF} \text { for radionuclide } i \text { for inhalation of nominal mass loading } \\
& \text { following a volcanic eruption (Sv/yr per } \mathrm{Bq} / \mathrm{m}^{2} \text { ) }
\end{aligned}
$$


Equations shown above use SI units for consistency with the documentation of the ERMYN model (BSC 2004 [DIRS 169460]). However, any units can be used to define parameters in GoldSim, where the ERMYN model is implemented, as long as they are dimensionally consistent. In the previous assessments, the output of the BDCF calculations was presented in units of rem $/ \mathrm{yr}$ per $\mathrm{pCi} / \mathrm{m}^{2}$, and this analysis follows the same format.

\subsubsection{Results of the Calculations}

The outcome of the biosphere model for the volcanic ash exposure scenario consists of the three BDCF components generated for each radionuclide and each model realization ( 3 radionuclidedependent BDCF components $\times 1,000$ realizations per radionuclide $\times(23+2)$ radionuclides $=$ 75,000 data points). A summary of these results, in the form of discrete cumulative probability distributions of BDCFs in 5 percentile increments, plus means and standard deviations, is presented in Tables 6.2-1 to 6.2-3 for the ICRP 30-based dosimetry and in Tables 6.2-4 to 6.2-6 for the ICRP 72-based dosimetry, respectively, for the external-ingestion-radon component, short-term inhalation component, and the long-term inhalation component, respectively. The values listed in Tables 6.2-1 to 6.2-6 are presented with three significant digits although only two significant digits are significant. This is to avoid round-off errors in the TSPA calculation of the annual dose. The BDCFs can be considered as intermediate results and, as such, should be given with an additional significant digit.

The list of biosphere model files is provided in Appendix A. The means, standard deviations, and percentiles were calculated in an Excel spreadsheet, $V A$ BDCF Realizations $M C \_$Rev 4.xls, for both ICRP 30 and ICRP 72 dosimetry. This file is described in Appendix B.

In all tables presenting the results of BDCF calculations, BDCFs for ${ }^{232} \mathrm{Th}$ include contributions from ${ }^{228} \mathrm{Ra}$ and ${ }^{228} \mathrm{Th}$; BDCFs for ${ }^{232} \mathrm{U}$ include the contribution from ${ }^{228} \mathrm{Th}$. 


\begin{tabular}{|c|c|c|c|c|c|c|c|c|c|c|c|c|c|c|c|c|c|c|c|c|c|c|c|}
\hline ల్ల & $\begin{array}{l}\infty \\
0 \\
\dot{1} \\
\dot{\alpha} \\
\infty \\
0 \\
0\end{array}$ & 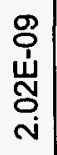 & 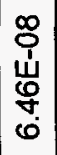 & 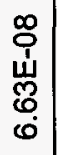 & 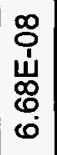 & 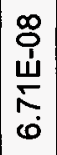 & 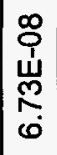 & 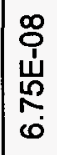 & 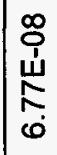 & 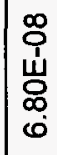 & 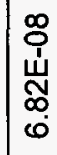 & 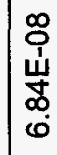 & 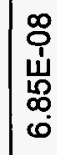 & 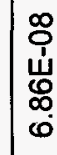 & $\mid \begin{array}{c}\infty \\
o \\
1 \\
\text { 岁 } \\
\infty \\
0 \\
0\end{array}$ & 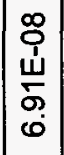 & 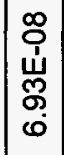 & 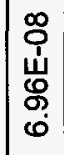 & 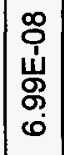 & 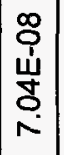 & 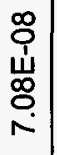 & 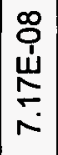 & 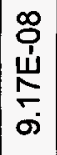 \\
\hline$\frac{0}{0}$ & 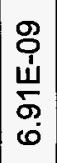 & $\begin{array}{l}\stackrel{8}{\circ} \\
\text { யे } \\
\text { هั } \\
\dot{0}\end{array}$ & 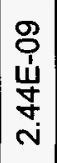 & 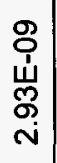 & 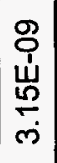 & 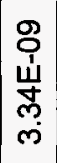 & 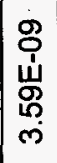 & 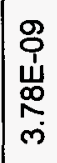 & 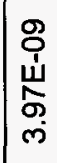 & 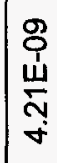 & $\begin{array}{l}\text { g } \\
\text { 㟧 } \\
\text { ơ } \\
\text { f }\end{array}$ & 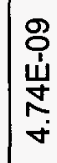 & 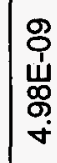 & 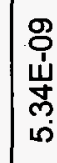 & 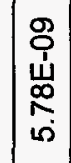 & 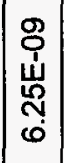 & 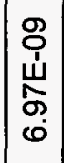 & $\mid \begin{array}{l}\stackrel{8}{o} \\
\stackrel{+}{W} \\
\stackrel{N}{N} \\
\end{array}$ & 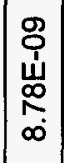 & 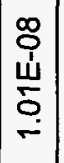 & 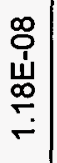 & 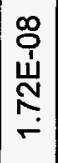 & 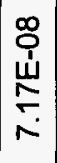 \\
\hline 랙 & 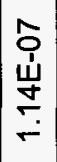 & $\begin{array}{l}\stackrel{8}{0} \\
\stackrel{4}{\circ} \\
\stackrel{8}{\circ} \\
\text { ஸे }\end{array}$ & 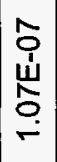 & 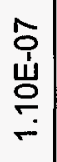 & 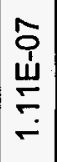 & 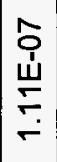 & 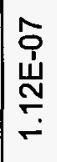 & 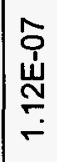 & 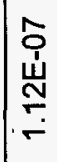 & 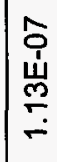 & 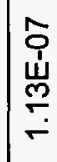 & 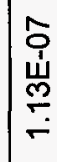 & 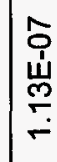 & 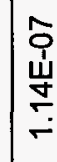 & 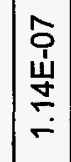 & 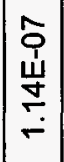 & 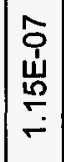 & 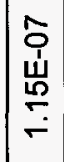 & 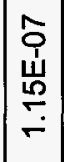 & $\begin{array}{c}\hat{o} \\
\dot{u} \\
\stackrel{u}{0} \\
\stackrel{-}{-}\end{array}$ & 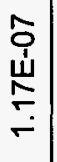 & 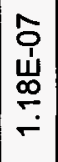 & 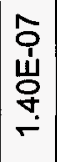 \\
\hline f & 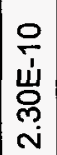 & 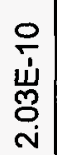 & $\begin{array}{l}\check{\sigma} \\
\dot{\leftrightarrow} \\
\emptyset \\
\emptyset \\
\omega\end{array}$ & 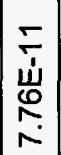 & 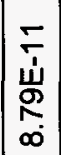 & 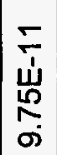 & 움 & 음 & 음 & 号 & 움 & 울 & 울 & 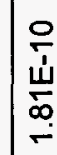 & 㑒 & 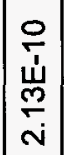 & 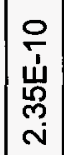 & $\mid \begin{array}{l}\text { 움 } \\
\dot{u} \\
o \\
o \\
\text { in }\end{array}$ & 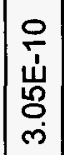 & 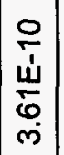 & 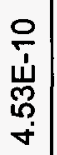 & 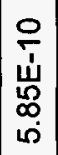 & 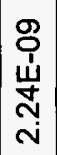 \\
\hline$\underset{\mathbb{E}}{\mathcal{F}}$ & 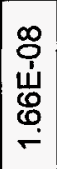 & 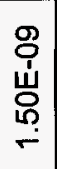 & 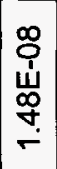 & 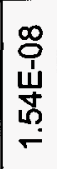 & 总 & 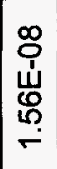 & 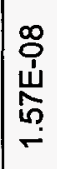 & 足 & 另 & 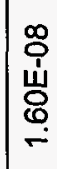 & 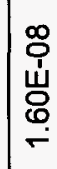 & 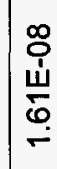 & 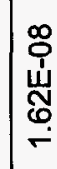 & 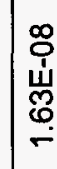 & 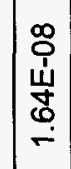 & 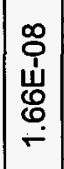 & 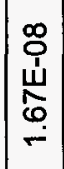 & 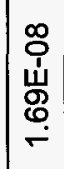 & 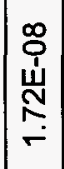 & 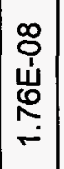 & 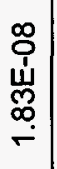 & $\begin{array}{l}\text { o } \\
\text { 岕 } \\
\text { ळু } \\
\stackrel{-}{-}\end{array}$ & 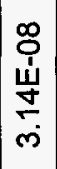 \\
\hline$\underset{\text { S }}{4}$ & 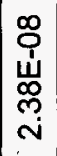 & 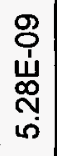 & 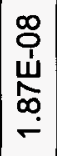 & 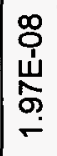 & 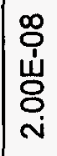 & 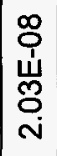 & 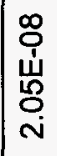 & $\begin{array}{l}\infty \\
0 \\
\text { ú } \\
\infty \\
0 \\
\text { v }\end{array}$ & 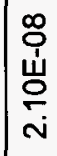 & 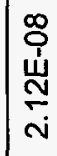 & 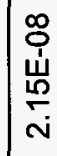 & 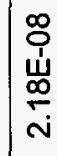 & $\begin{array}{c}\text { o } \\
\text { ư } \\
\text { Ny } \\
\text { c }\end{array}$ & 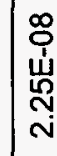 & 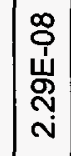 & 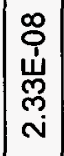 & 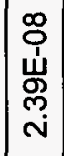 & 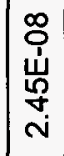 & 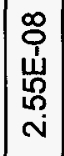 & 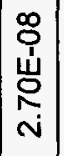 & 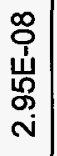 & 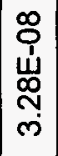 & 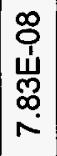 \\
\hline$\ddot{\alpha}$ & $\begin{array}{c}\stackrel{\sigma}{+} \\
\stackrel{\leftrightarrow}{\omega} \\
\stackrel{6}{-}\end{array}$ & 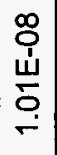 & 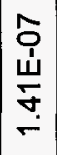 & 方 & 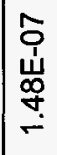 & 容 & 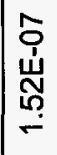 & 容 & 号 & 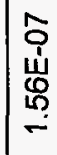 & 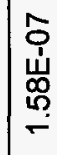 & 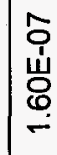 & $\begin{array}{l}\mathfrak{o} \\
\text { w. } \\
\check{c} \\
\stackrel{2}{\sigma}\end{array}$ & 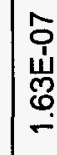 & 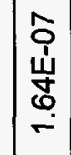 & 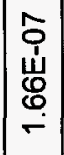 & 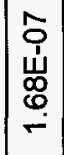 & 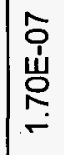 & 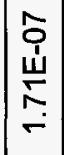 & 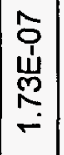 & 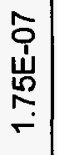 & 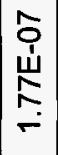 & 옴 \\
\hline 0 & 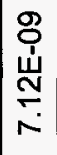 & 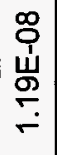 & $\begin{array}{l}\text { 음 } \\
\text { ய் } \\
\dot{\infty} \\
\infty \\
\dot{0}\end{array}$ & 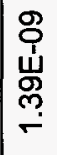 & 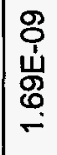 & 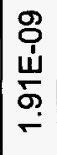 & 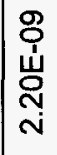 & 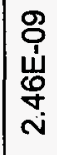 & 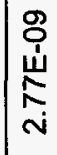 & 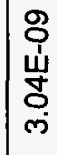 & 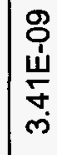 & 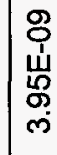 & 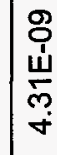 & 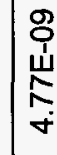 & 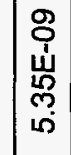 & 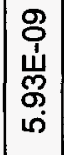 & 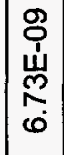 & 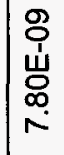 & 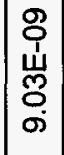 & 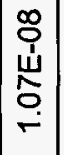 & 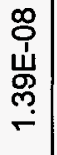 & 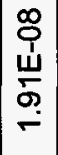 & 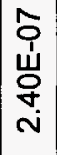 \\
\hline \& & 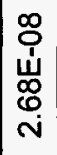 & $\begin{array}{l}\stackrel{0}{\dot{1}} \\
\dot{\omega} \\
\\
\dot{0}\end{array}$ & 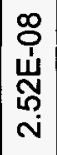 & 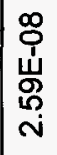 & 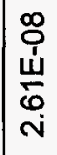 & 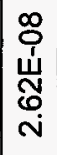 & 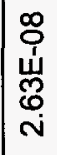 & 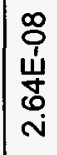 & 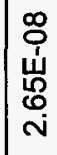 & 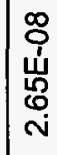 & $\begin{array}{l}\infty \\
0 \\
0 \\
\dot{u} \\
0 \\
0 \\
0 \\
\text { v }\end{array}$ & 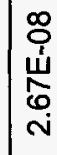 & 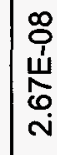 & 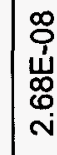 & $\begin{array}{l}\infty \\
\text { o } \\
\text { ư } \\
\text { og } \\
\text { ஸ่ }\end{array}$ & 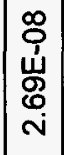 & 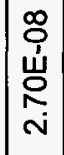 & 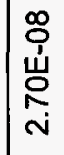 & 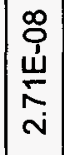 & $\begin{array}{c}\infty \\
0 \\
\stackrel{4}{W} \\
\stackrel{N}{N} \\
i\end{array}$ & 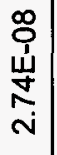 & 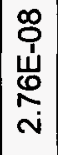 & 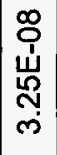 \\
\hline 5 & 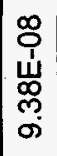 & 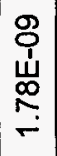 & 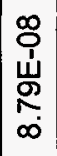 & 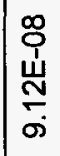 & 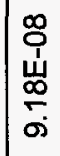 & 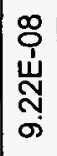 & 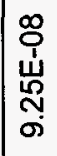 & 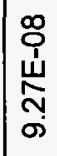 & 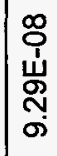 & 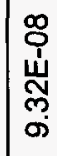 & $\begin{array}{l}\infty \\
\text { o } \\
\text { யُ } \\
\text { m } \\
\sigma\end{array}$ & $\begin{array}{l}\infty \\
0 \\
0 \\
4 \\
0 \\
ల \\
\infty \\
\infty\end{array}$ & $\begin{array}{c}\infty \\
0 \\
\text { p. } \\
\text { w } \\
\text { o } \\
\sigma\end{array}$ & $\begin{array}{l}\infty \\
\text { o } \\
\text { u் } \\
\text { o } \\
\text { o }\end{array}$ & 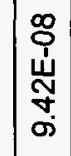 & 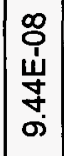 & 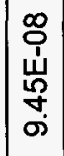 & 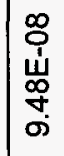 & $\mid \begin{array}{l}\infty \\
0 \\
\dot{d} \\
\grave{\omega} \\
\infty \\
\infty\end{array}$ & 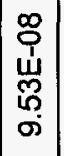 & $\begin{array}{l}\infty \\
o \\
\dot{w} \\
\text { Tn } \\
\text { on }\end{array}$ & 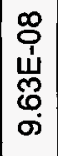 & 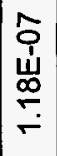 \\
\hline ஓ & 울 & 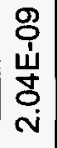 & 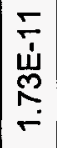 & 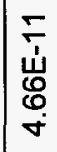 & 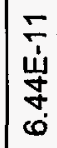 & 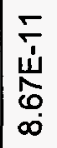 & 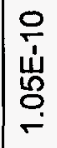 & 음 & 움 & 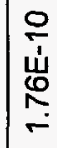 & $\frac{0}{\text { 넘 }}$ & 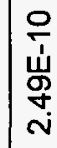 & 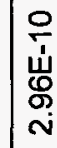 & 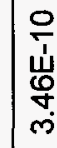 & 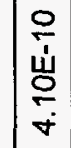 & 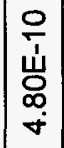 & 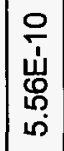 & 음 & 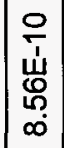 & 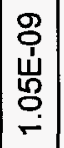 & 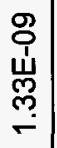 & $\begin{array}{c}\stackrel{g}{o} \\
\stackrel{+}{w} \\
\stackrel{5}{\sigma} \\
\stackrel{-}{-}\end{array}$ & 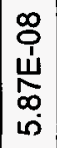 \\
\hline & 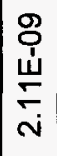 & 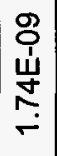 & 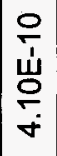 & 움 & 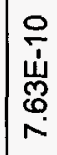 & $\begin{array}{l}\text { 움 } \\
\text { 양 } \\
\infty \\
\infty\end{array}$ & $\begin{array}{l}\text { 움 } \\
\text { 山े } \\
\text { के }\end{array}$ & 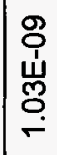 & 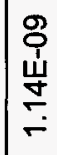 & $\begin{array}{l}\text { o } \\
\text { 嵓 } \\
\text { N̦ }\end{array}$ & 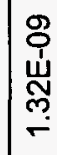 & 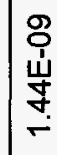 & 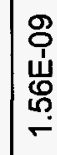 & 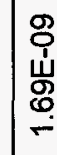 & 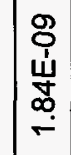 & 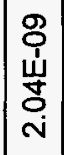 & 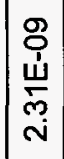 & 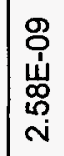 & 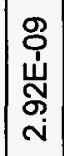 & 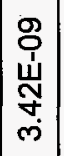 & 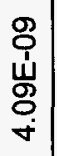 & 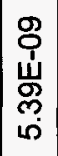 & 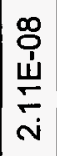 \\
\hline & $\begin{array}{l}\stackrel{c}{\mathbb{D}} \\
\stackrel{\mathbb{E}}{\Sigma}\end{array}$ & 号 & 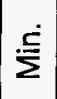 & ㅇํㅁ & ஃ̊ํ & 웅 & ઠ̊ํ & $\stackrel{i n}{N}$ & ઠ̊̊ & फ्रे & q & f & ه & 饮 & ठి & ㅇํㅇ & ’̊요 & 䓍 & 今̊ & 总 & ঃ̊ & ㅇํㅇ & $\sum^{\dot{x}}$ \\
\hline
\end{tabular}




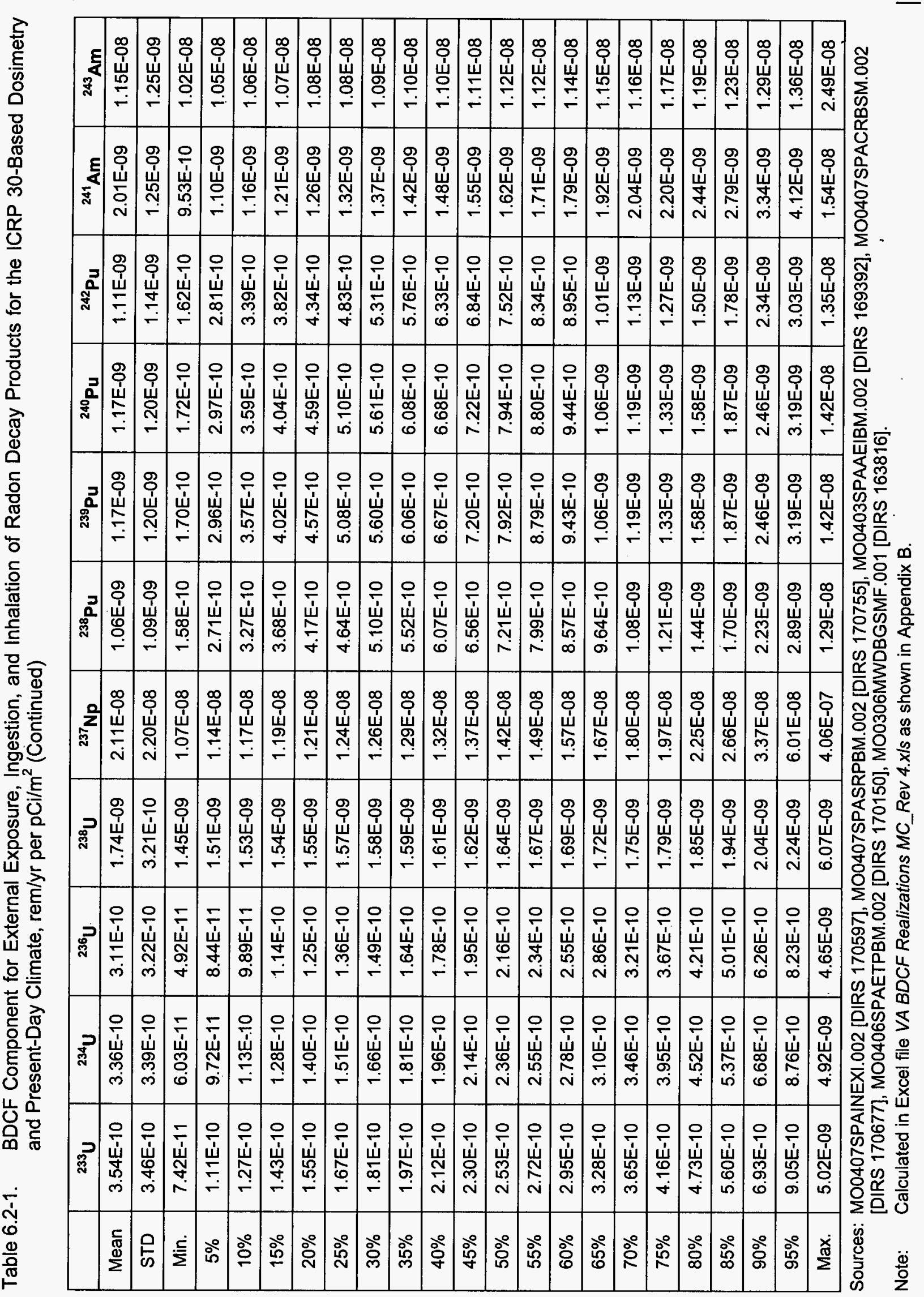




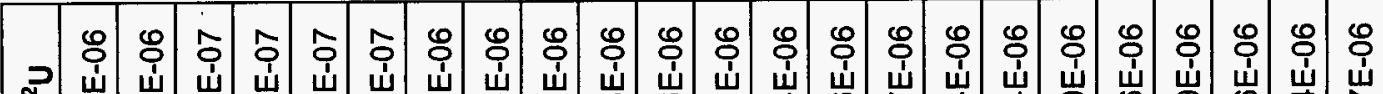

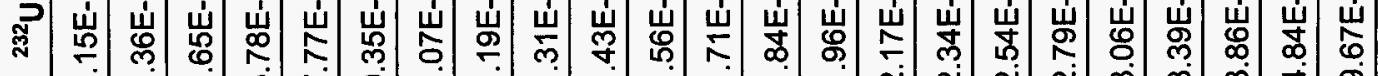

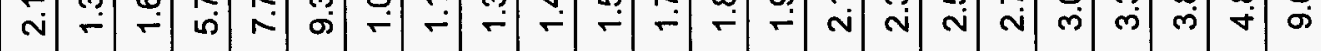

잉

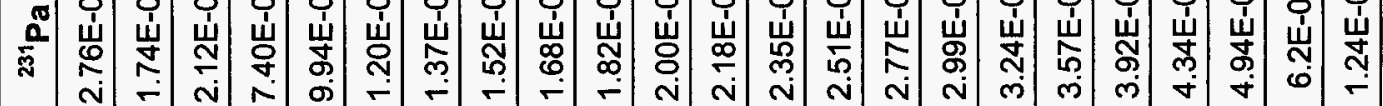

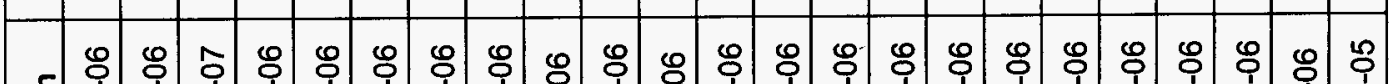

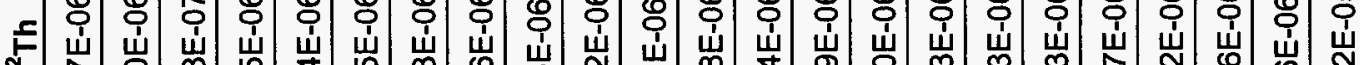

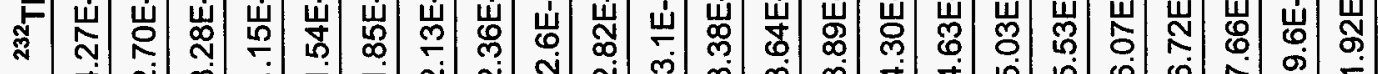

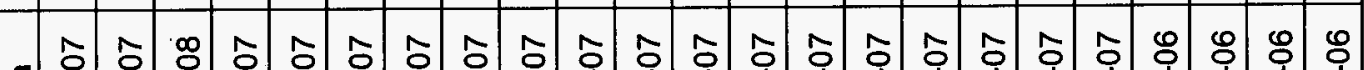

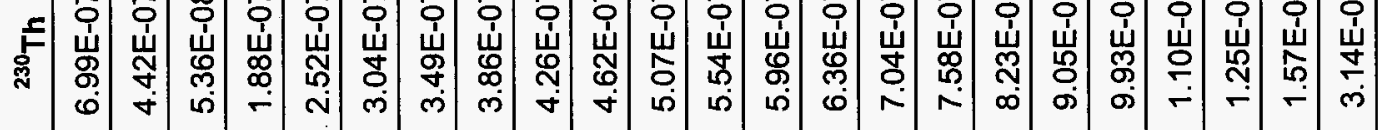

:

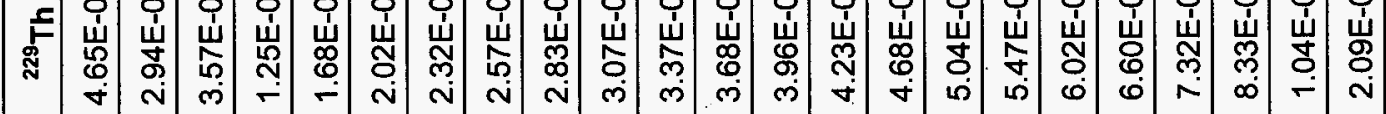
ón

$\frac{a}{0}$

$\underline{\underline{u}}$

$\stackrel{\Phi}{\rightleftharpoons}$

흔

这

:

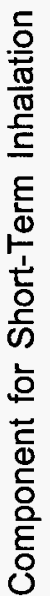
岂色

○ ₹

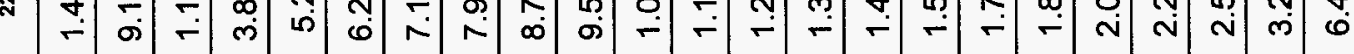

๑

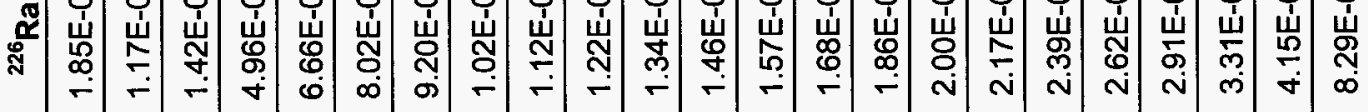

م

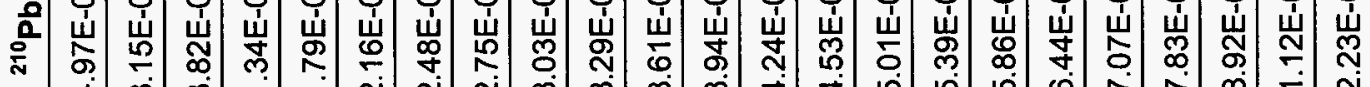

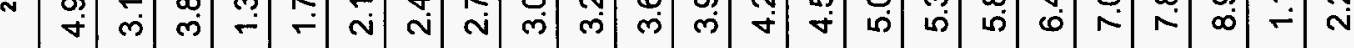

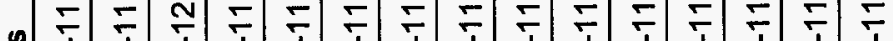

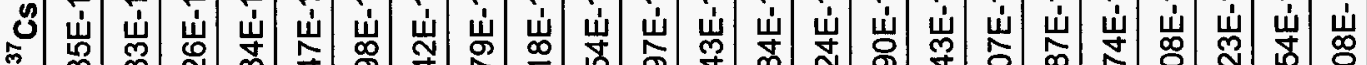

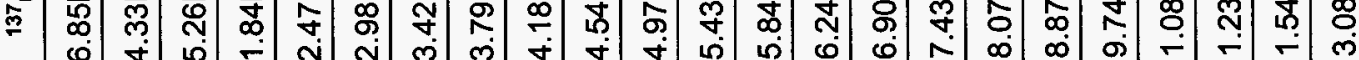

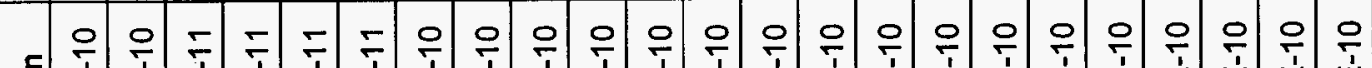

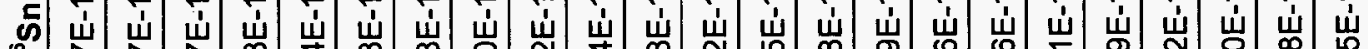

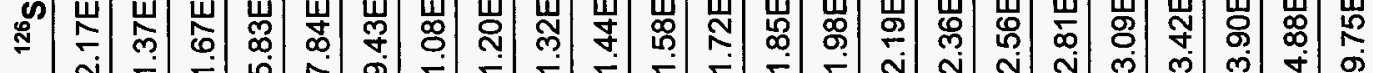

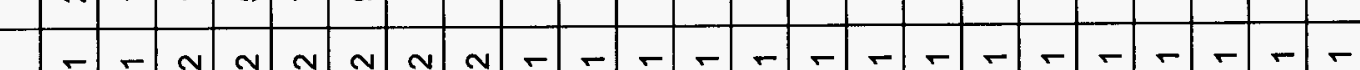

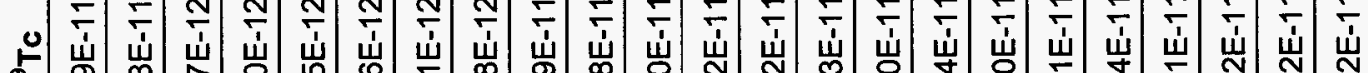

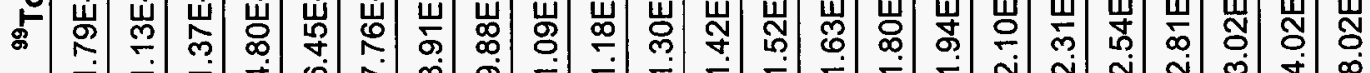

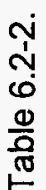

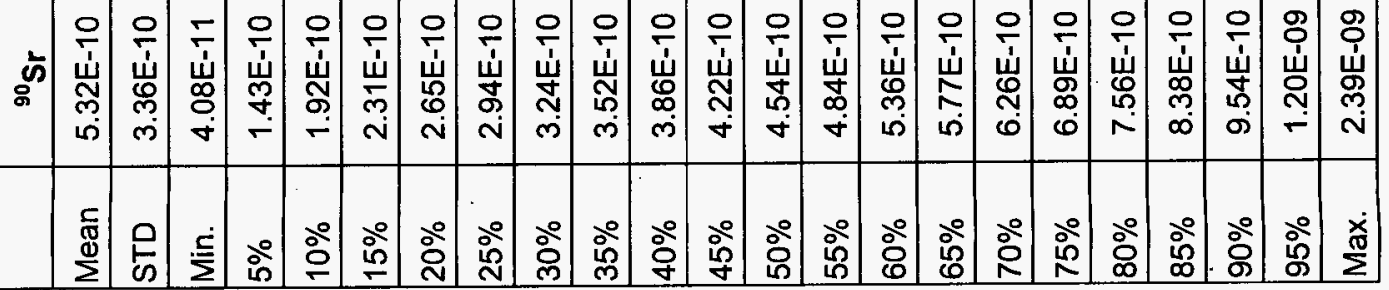




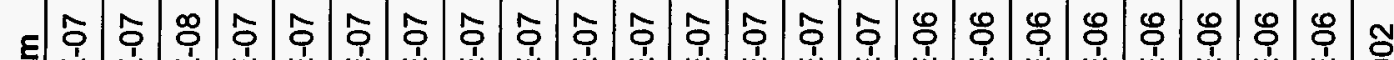
g O)

E

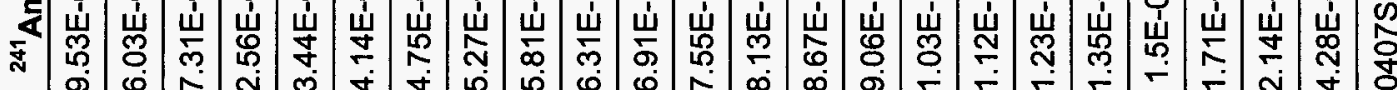

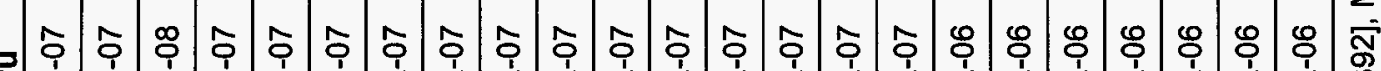
0

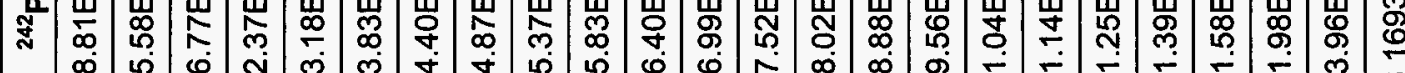

人ิ 율 嵌

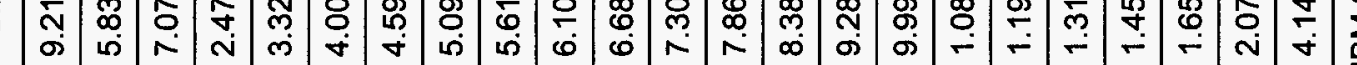

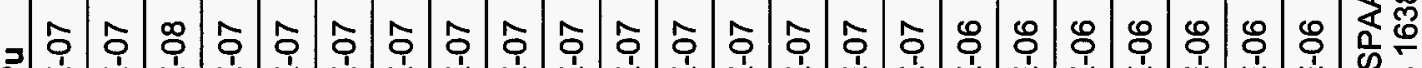
可 嵌

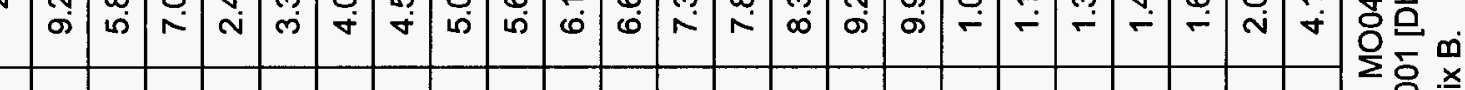

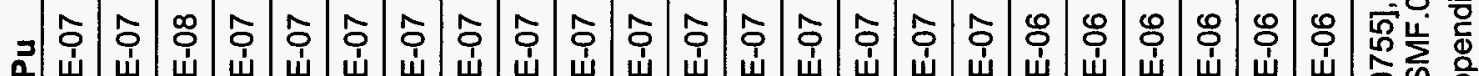

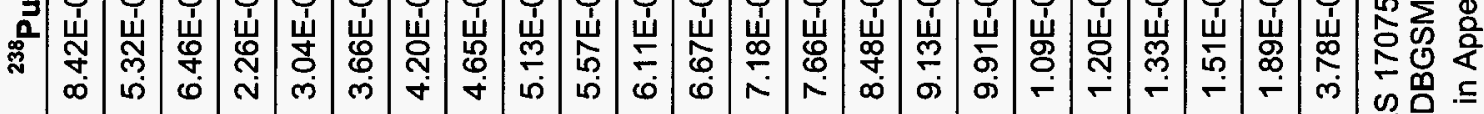

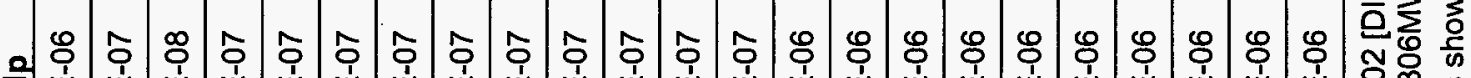

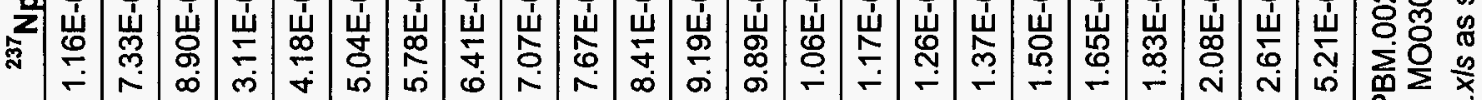

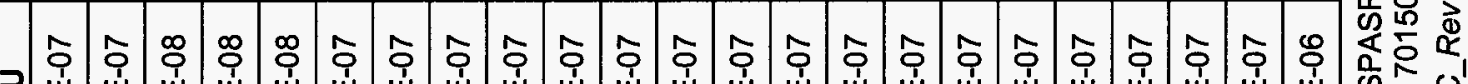

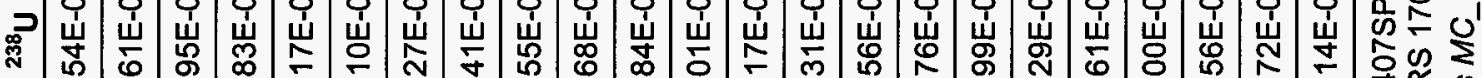

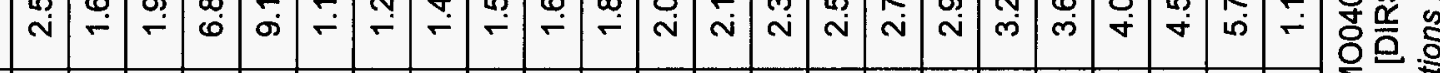

梂全

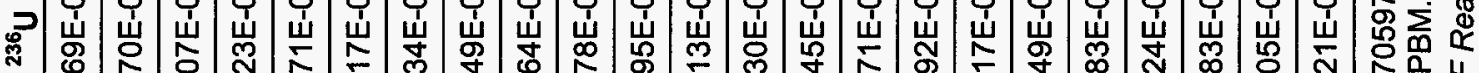
후

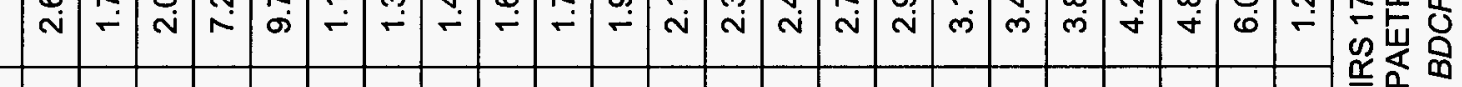

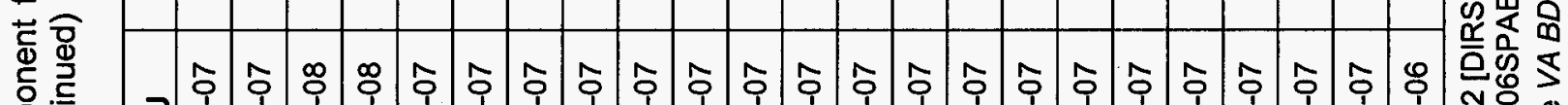

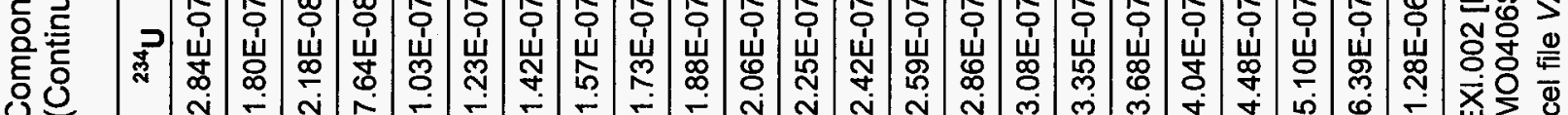
Uำ 品

(1)

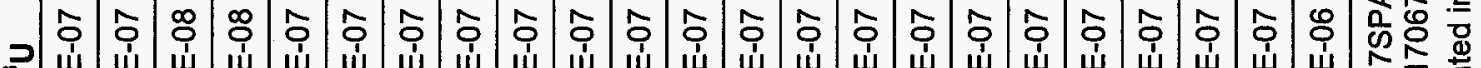

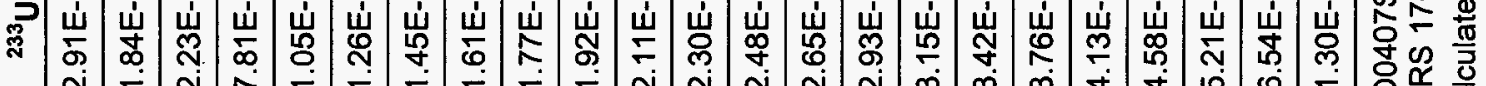

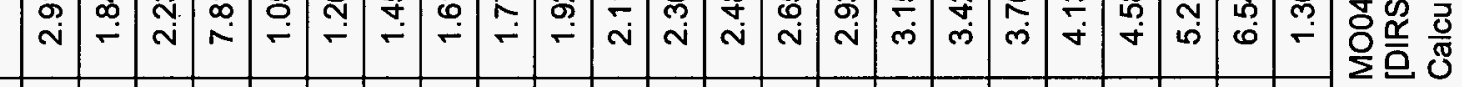

ஸे

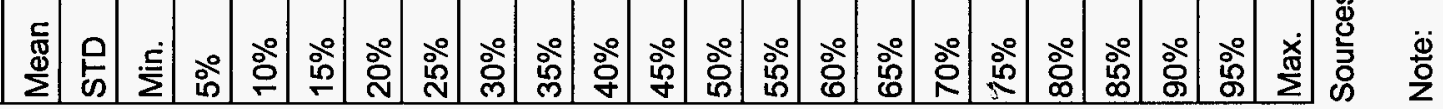




\begin{tabular}{|c|c|c|c|c|c|c|c|c|c|c|c|c|c|c|c|c|c|c|c|c|c|c|c|}
\hline ฉ్లై & 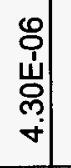 & 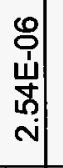 & 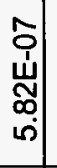 & 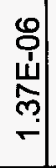 & 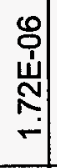 & 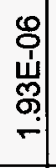 & 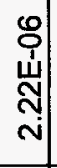 & $\begin{array}{l}\text { ơ } \\
\text { ư } \\
\text { ஸे } \\
\text { ஸे }\end{array}$ & 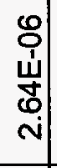 & 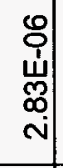 & 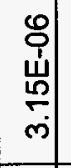 & 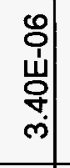 & 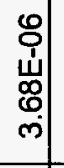 & 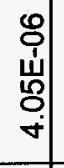 & 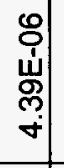 & 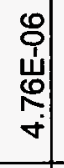 & 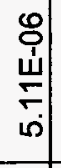 & 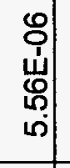 & 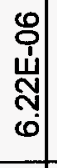 & 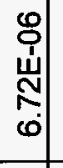 & 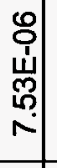 & 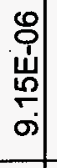 & 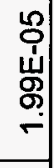 \\
\hline$\frac{0}{n}$ & 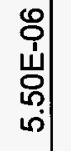 & 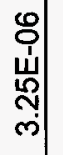 & 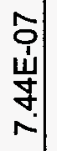 & 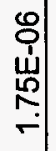 & $\begin{array}{l}\text { 山्ग } \\
\text { ㄱ. }\end{array}$ & 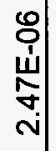 & 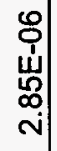 & 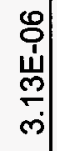 & 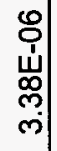 & 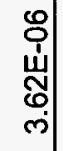 & 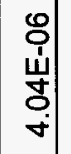 & 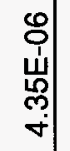 & 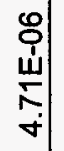 & 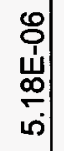 & $\begin{array}{l}\text { ய் } \\
\text { ம் }\end{array}$ & 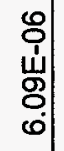 & 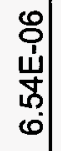 & 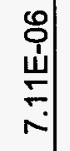 & 岕 & $\begin{array}{l}0 \\
\vdots \\
\text { ப் } \\
0 \\
\infty \\
\infty\end{array}$ & 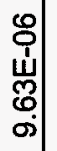 & 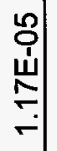 & $\underset{⿱ 乛 龰}{u}$ \\
\hline$\stackrel{5}{5}$ & $\begin{array}{c}\text { U్ } \\
\text { W. } \\
\text { స్. } \\
\infty\end{array}$ & $\begin{array}{l}\text { 岗 } \\
\text { iे }\end{array}$ & 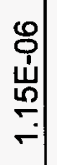 & 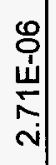 & $\begin{array}{l}\vec{U} \\
\dot{v} \\
\dot{m}\end{array}$ & 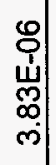 & 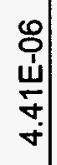 & 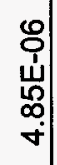 & 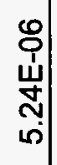 & 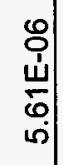 & 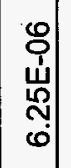 & 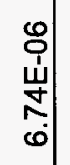 & 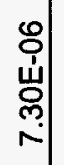 & 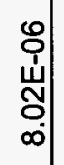 & $\begin{array}{l}7 \\
\infty\end{array}$ & $\begin{array}{l}\text { 岧} \\
\text { ó }\end{array}$ & 5 & 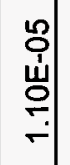 & | & $\stackrel{\leftrightarrow}{\leftrightarrow}$ & 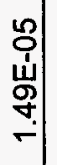 & -1 & \\
\hline 당 & 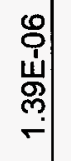 & 客 & 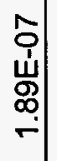 & 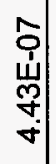 & 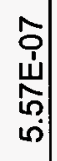 & 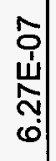 & $\begin{array}{l}\text { ồ } \\
\stackrel{4}{N} \\
\text { N̦ }\end{array}$ & 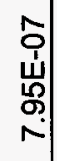 & $\begin{array}{l}\text { 어 } \\
\text { ú } \\
0 \\
0 \\
\infty \\
0\end{array}$ & 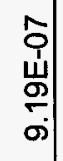 & 뻥 & 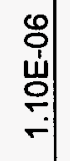 & 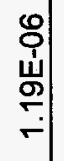 & 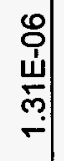 & 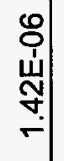 & 岁 & 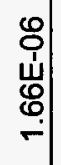 & 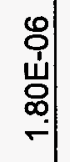 & 8 & 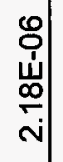 & 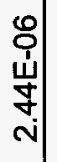 & $\frac{1}{3}$ & \\
\hline F & 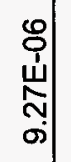 & 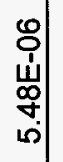 & 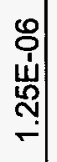 & 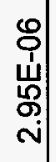 & 岂 & 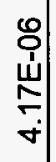 & $\begin{array}{l}0 \\
\stackrel{1}{1} \\
\stackrel{्}{0} \\
0 \\
\dot{+}\end{array}$ & 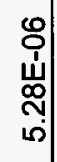 & 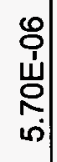 & 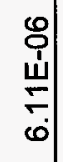 & 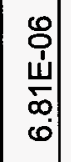 & 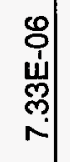 & 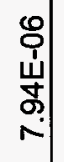 & 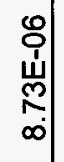 & $\stackrel{\omega}{N}$ & 岗 & 恧 & $\begin{array}{l}\text { Oे } \\
\text { 山్ } \\
\text { ำ }\end{array}$ & 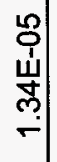 & $\stackrel{\text { L }}{\stackrel{5}{-}}$ & $\stackrel{\mathcal{g}}{-}$ & 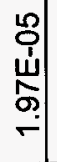 & \\
\hline${ }_{N}^{0}$ & 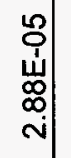 & 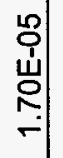 & 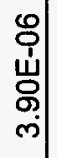 & 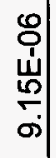 & 㸃 & 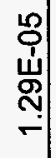 & $\stackrel{\text { 山े }}{\stackrel{9}{+}}$ & 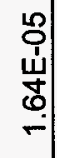 & 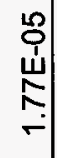 & 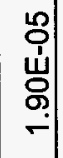 & $\stackrel{\varpi}{\mp}$ & $\mid \begin{array}{c}\text { 㟧} \\
\mid\end{array}$ & i. & $\frac{\omega}{\widehat{N}}$ & 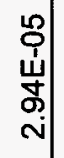 & $\begin{array}{l}\stackrel{\dot{⿱ ⺌ 冖 ⿱ 二 厶}}{\dot{m}} \\
\end{array}$ & 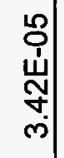 & 岕 & 总 & 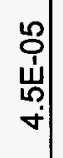 & 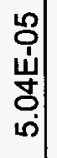 & i| & \\
\hline 莡 & 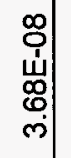 & 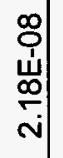 & $\begin{array}{l}\text { 山े } \\
\text { o } \\
\stackrel{\text { v }}{*}\end{array}$ & 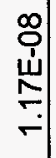 & 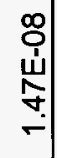 & $\begin{array}{l}\infty \\
0 \\
\dot{u} \\
\stackrel{0}{0} \\
\stackrel{0}{0}\end{array}$ & $\stackrel{\text { ஸ் }}{\stackrel{\sigma}{-}}$ & 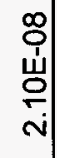 & 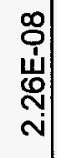 & 岕 & 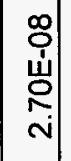 & 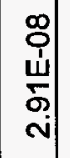 & 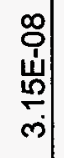 & $\begin{array}{c}\vec{W} \\
y \\
\dot{m}\end{array}$ & 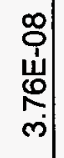 & 岁 & $\begin{array}{l}\dot{u} \\
\underset{\infty}{0} \\
\forall\end{array}$ & 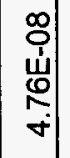 & 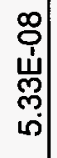 & مे & . & | & \\
\hline$\frac{0}{0}$ & 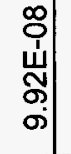 & 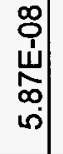 & 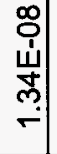 & 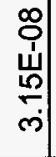 & $\begin{array}{l}\text { ơ } \\
\text { ự }\end{array}$ & $\begin{array}{l}\text { o } \\
\stackrel{+}{ } \\
\stackrel{u}{0} \\
\stackrel{+}{+}\end{array}$ & 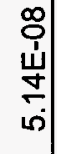 & 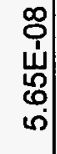 & 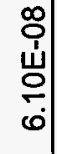 & 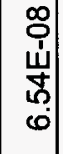 & 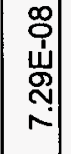 & $\mid \begin{array}{c}0 \\
0 \\
\text { 山⿱山口 } \\
\text { | }\end{array}$ & 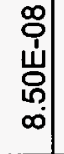 & 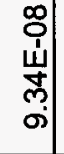 & 옴 & 인 & $\begin{array}{c}\text { o } \\
\text { 岕 } \\
\text { no }\end{array}$ & 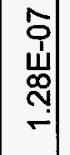 & 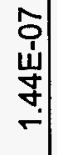 & 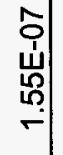 & -1 & 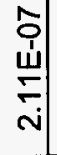 & \\
\hline 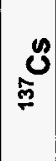 & 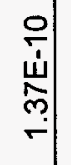 & 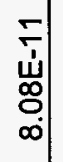 & 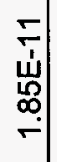 & 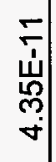 & 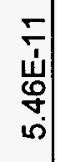 & 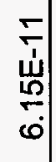 & 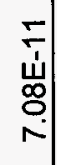 & 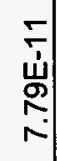 & 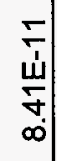 & $\begin{array}{l}\bar{\omega} \\
\bar{\omega} \\
\sigma \\
\sigma\end{array}$ & 岁 & $\left|\begin{array}{c}0 \\
\text { ㅇ. } \\
0 \\
0 \\
\circ\end{array}\right|$ & 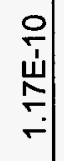 & 岕 & జ் & $\stackrel{\dot{w}}{\bar{n}}$ & 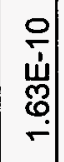 & 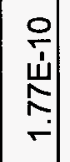 & -1 & 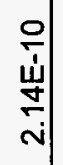 & |ج & பإ) & \\
\hline 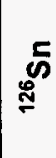 & 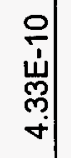 & 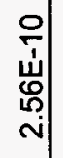 & 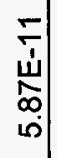 & 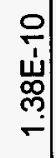 & 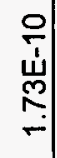 & $\begin{array}{l}\text { 은 } \\
\text { 山े } \\
\stackrel{\leftrightarrow}{-} \\
\stackrel{5}{-}\end{array}$ & 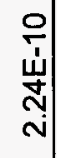 & 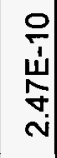 & 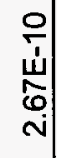 & 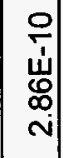 & 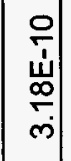 & 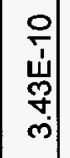 & 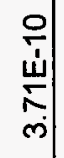 & 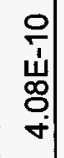 & 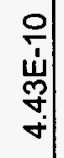 & 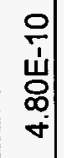 & 岁 & \begin{tabular}{|c}
$\omega$ \\
$\bar{\sigma}$
\end{tabular} & 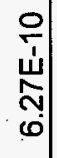 & مإ. & ì| & $\begin{array}{l}\text { ஸ़ } \\
\text { ஸ̆ }\end{array}$ & \\
\hline 5 & 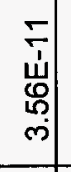 & 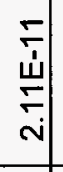 & 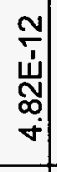 & 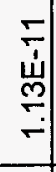 & $\begin{array}{c}\overline{\dot{u}} \\
\stackrel{\sim}{*}\end{array}$ & $\begin{array}{l}\bar{\leftarrow} \\
\dot{\leftrightarrow} \\
\stackrel{0}{-}\end{array}$ & 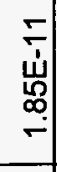 & 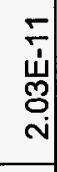 & 岀 & 岕 & ய் & 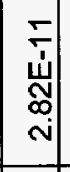 & $\begin{array}{l}\text { 岗 } \\
\text { ले } \\
\text { ले }\end{array}$ & | & 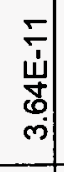 & 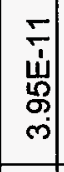 & 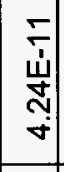 & щ் & 它 & 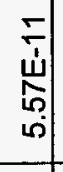 & 'ئ & 屌 & \\
\hline 8 & 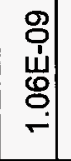 & 号 & 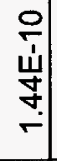 & 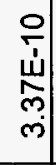 & 움 & 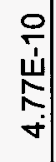 & \begin{tabular}{l} 
울 \\
$\dot{W}$ \\
\multirow{\sigma}{*}{}
\end{tabular} & 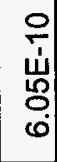 & 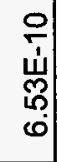 & 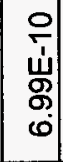 & 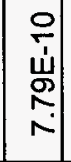 & $\mid \begin{array}{c}\circ \\
\text { ய் } \\
\text { 요 } \\
\infty\end{array}$ & 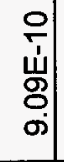 & $\begin{array}{l}\text { 山े. } \\
\text { ه̆ } \\
\sigma\end{array}$ & 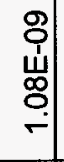 & 岕 & 㤐 & 崩 & 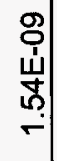 & ب. & 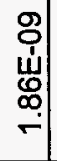 & 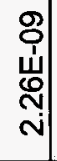 & 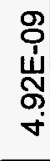 \\
\hline & $\bar{\Phi}$ & 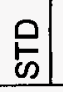 & $\Sigma$ & in & $=$ & $\stackrel{60}{5}$ & ని & $\stackrel{్}{\sim}$ & ల్ & 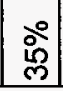 & 워 & 寸 & in & 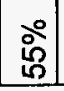 & 01 & 01 & & 을 & $\infty$ & $\infty$ & d) & o & \\
\hline
\end{tabular}


这

空

$\frac{1}{0}$

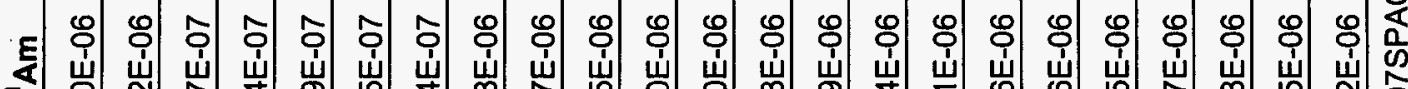

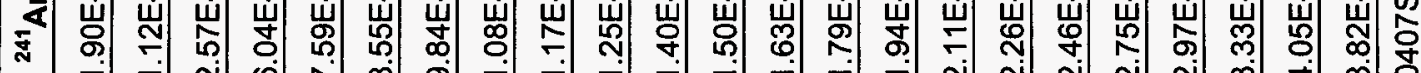

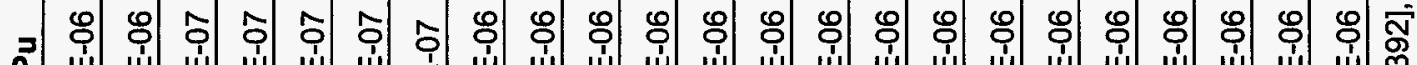

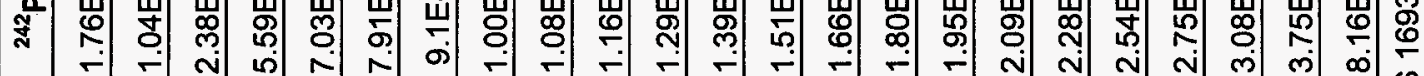

$\sum_{\frac{D}{0}}^{\frac{\pi}{0}}$

\&

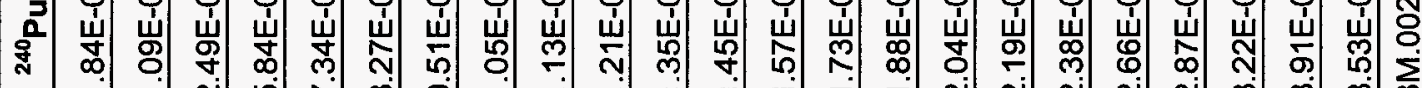

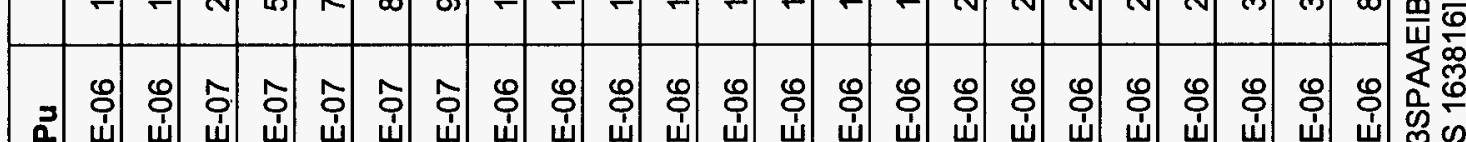

品

이

몽

$\stackrel{\Phi}{\rightleftarrows}$

훈

ֻัญ

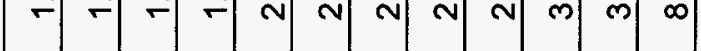

은

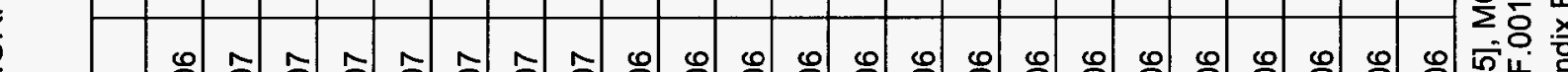

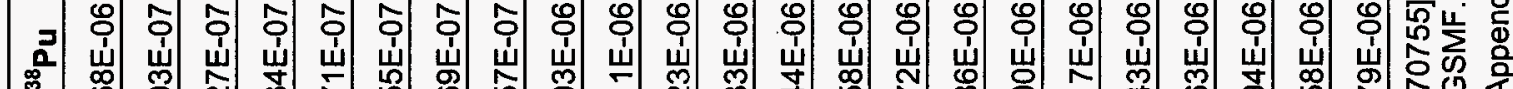

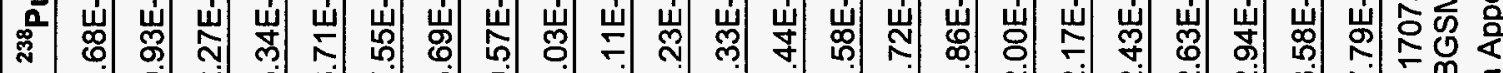

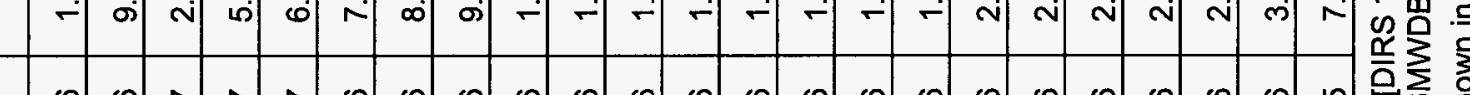

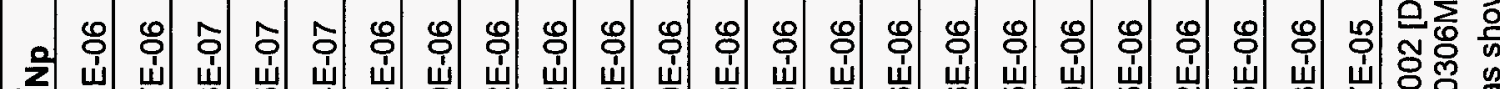

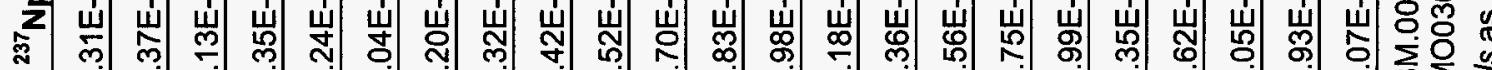
ஸे

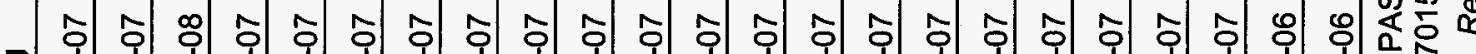

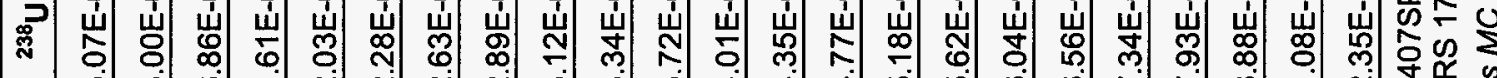

E⿱士⿻尸丨心

ம)

官

$\stackrel{\square}{\square}$

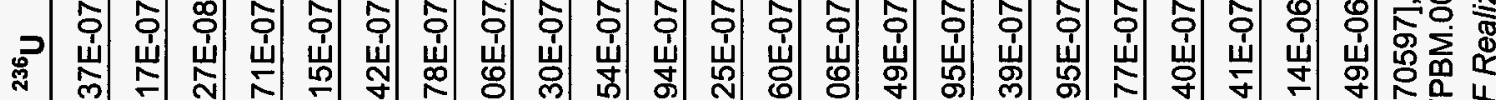

t艹

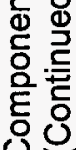

บั

吕 宮

$\ddot{\sim}$

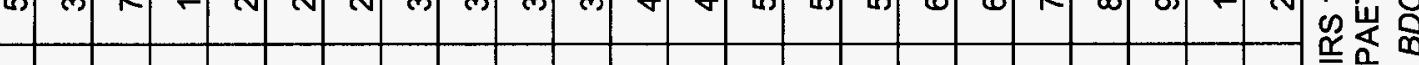

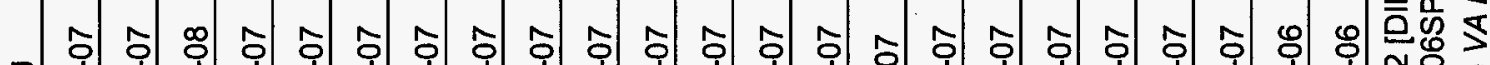

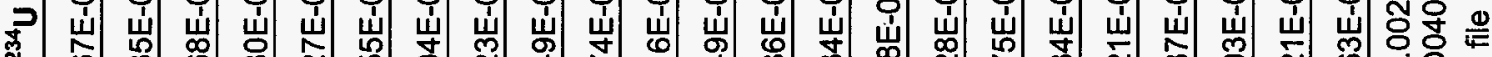

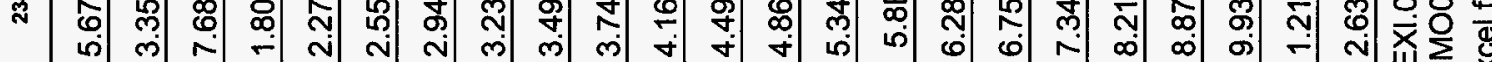

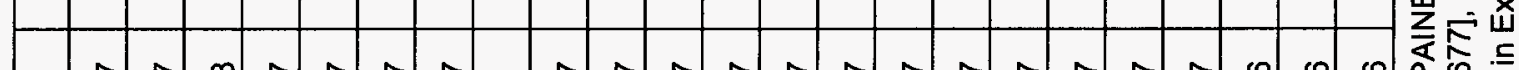

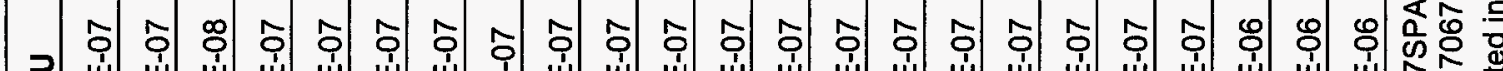

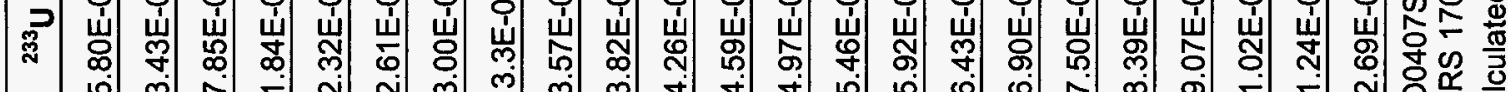

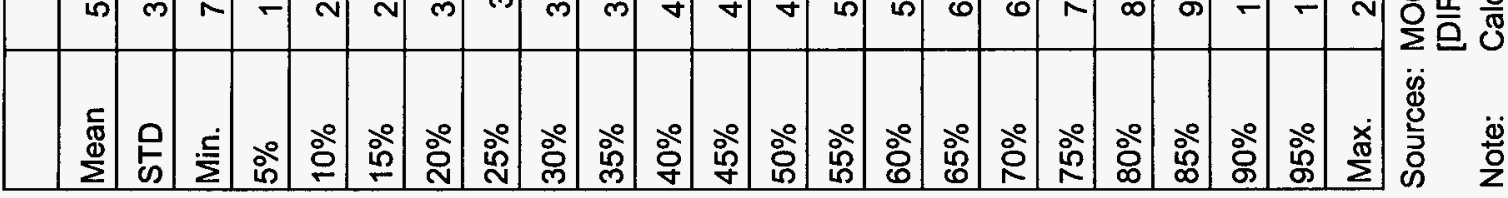




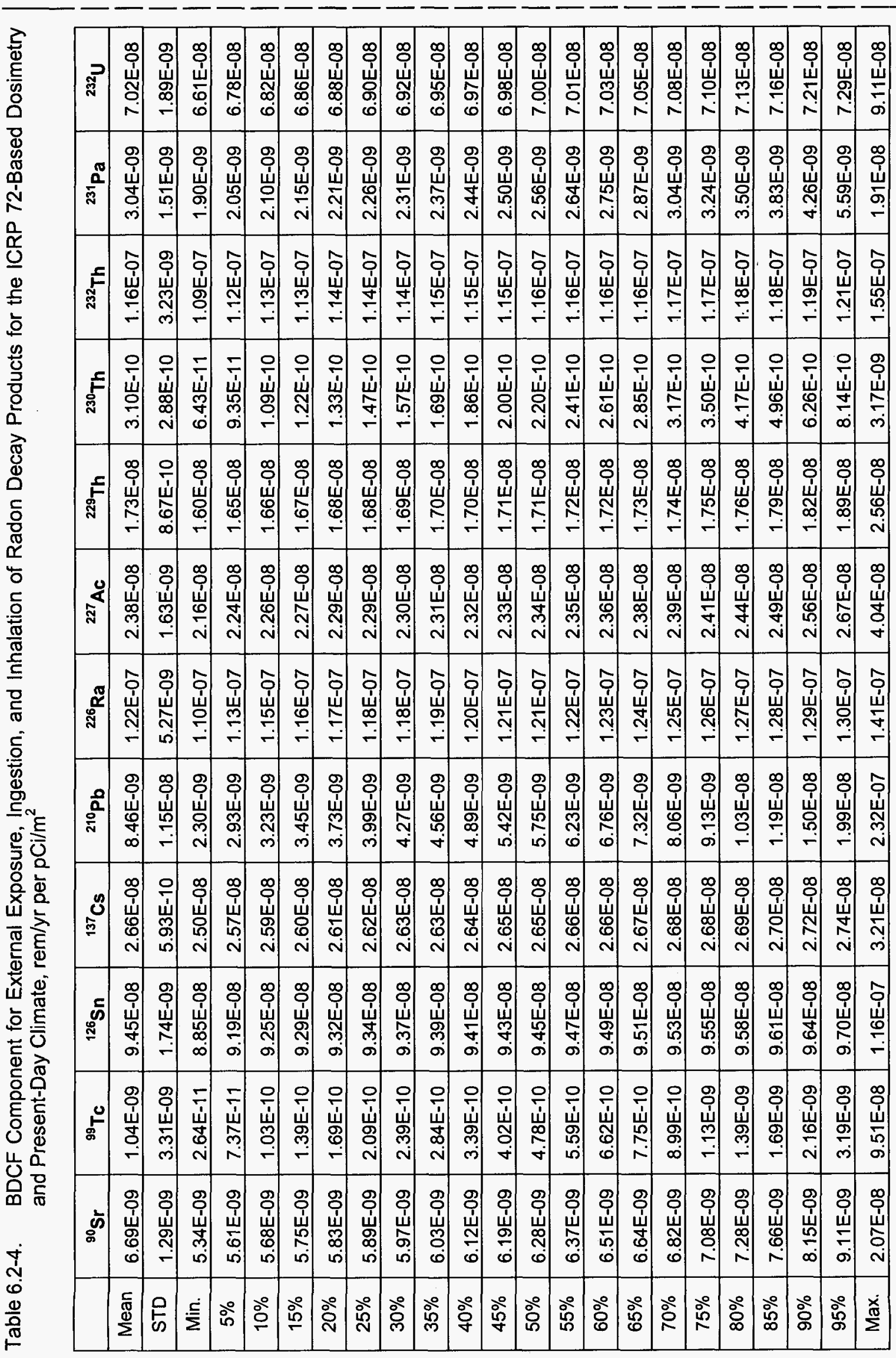




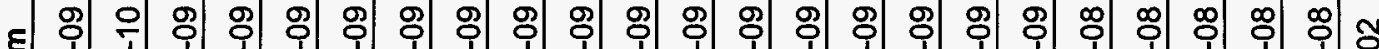

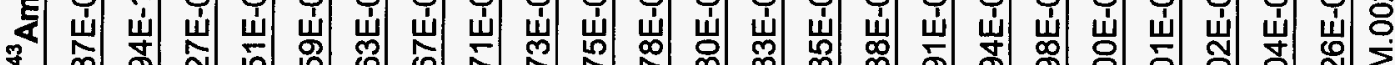

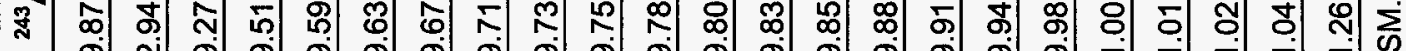

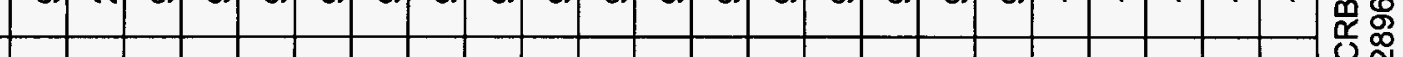

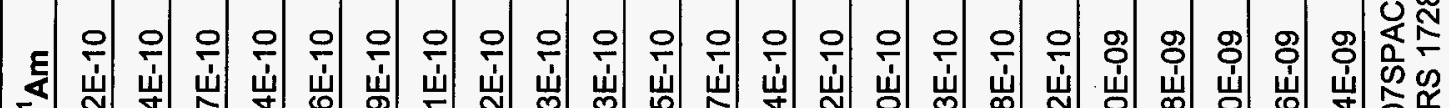

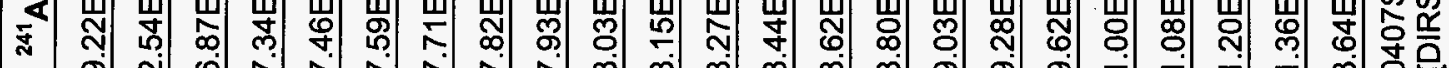

$\underline{\underline{\alpha}}$

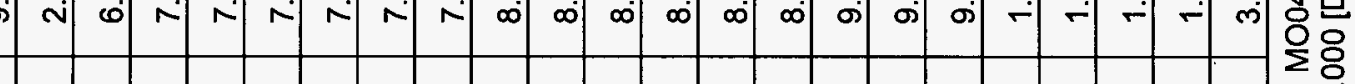

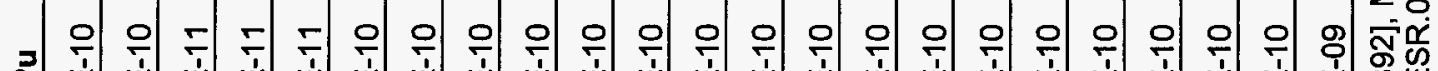

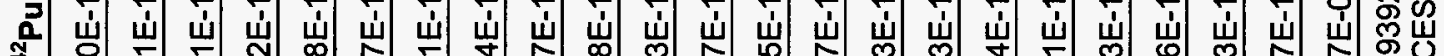

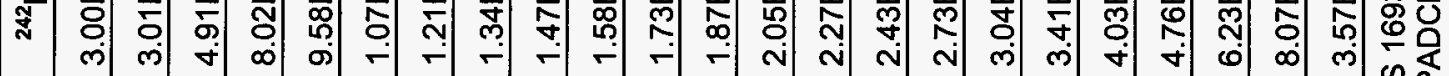

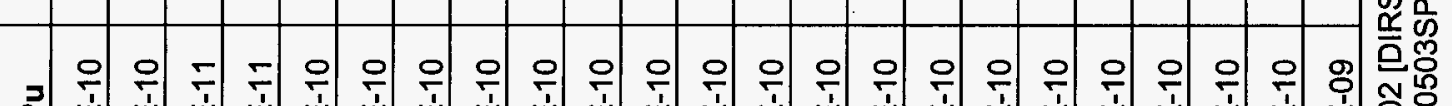

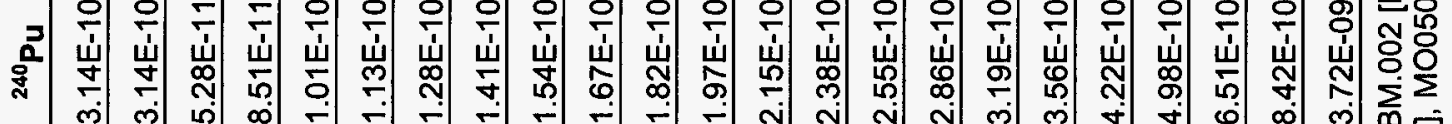

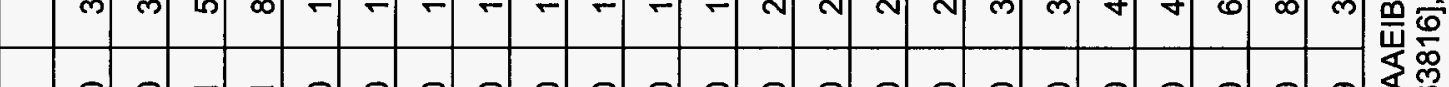

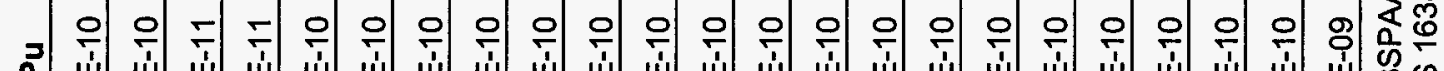

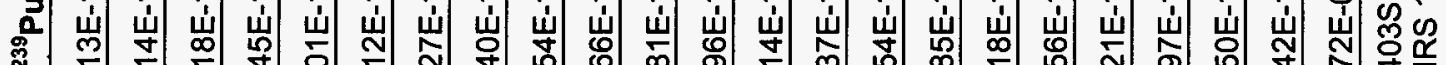

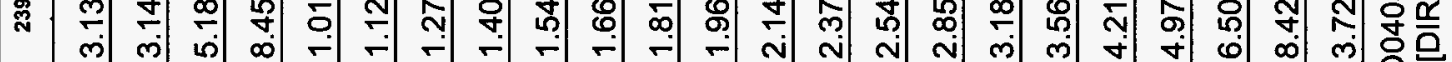

象

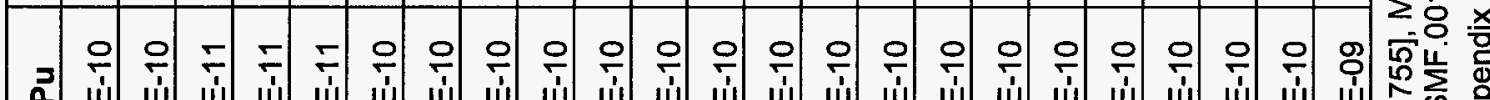

员 岁 岁

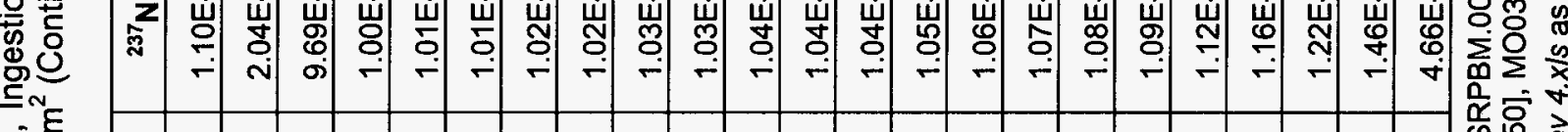

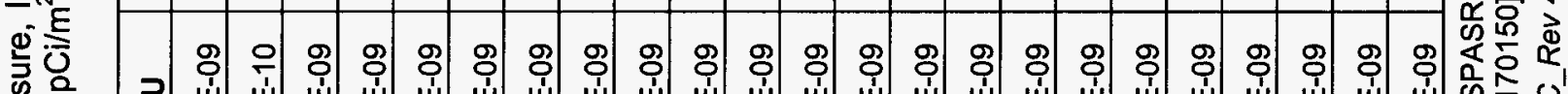

要

₹

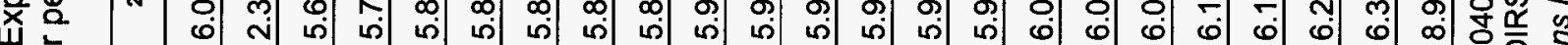

ত

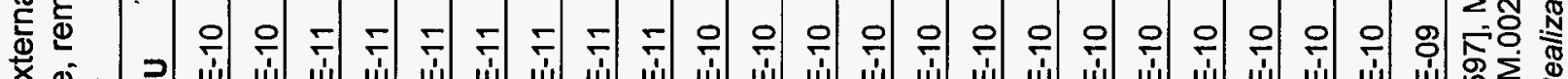

ய ब

ᄒ

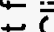

ब

응 눙

ठ용

岀

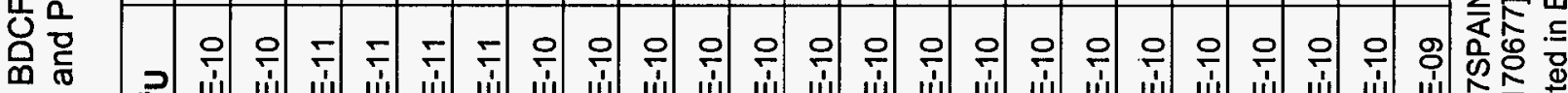

(1)

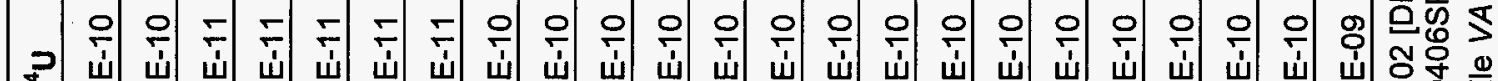

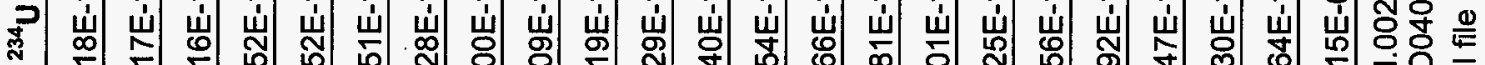

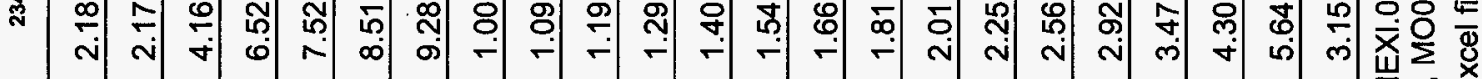

๊

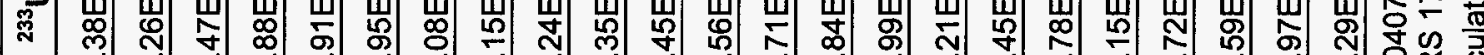

¿ั่

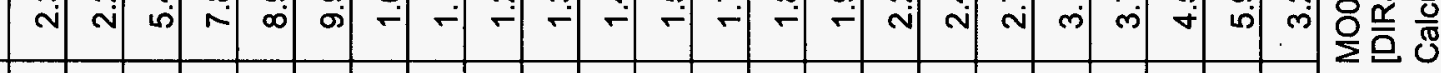

离

is

$\frac{\omega}{\frac{0}{0}}$ 


\begin{tabular}{|c|c|c|c|c|c|c|c|c|c|c|c|c|c|c|c|c|c|c|c|c|c|c|c|}
\hline ᄀי & $\begin{array}{l}0 \\
\text { ù } \\
0 \\
0 \\
0\end{array}$ & 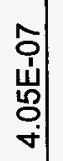 & 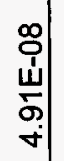 & $\begin{array}{l}\text { ợ } \\
\stackrel{u}{N} \\
\stackrel{ }{-}\end{array}$ & 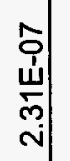 & 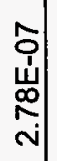 & 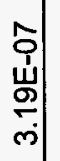 & 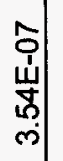 & 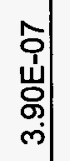 & $\begin{array}{c}\hat{O} \\
\stackrel{u}{\mathbf{U}} \\
\stackrel{+}{+}\end{array}$ & 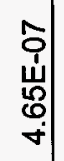 & 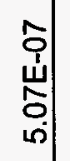 & 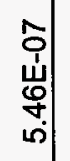 & 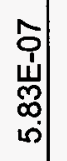 & $\begin{array}{l}\text { ợ } \\
\text { ư } \\
\text { ơ } \\
0\end{array}$ & 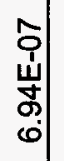 & $\begin{array}{l}\hat{0} \\
\text { ự } \\
\text { م. }\end{array}$ & 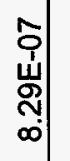 & $\begin{array}{l}\hat{0} \\
\text { 山్} \\
\text { ஸे } \\
\text { के }\end{array}$ & 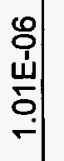 & 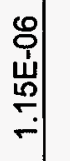 & 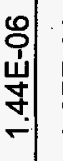 & 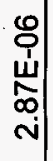 \\
\hline$\frac{\mathbb{2}}{80}$ & 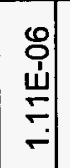 & 帘 & 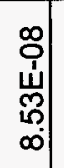 & $\begin{array}{l}\text { 위 } \\
\text { யे } \\
\text { o } \\
\text { ㄱ. }\end{array}$ & $\begin{array}{l}\text { 일 } \\
\text { யे } \\
\text { 후 }\end{array}$ & 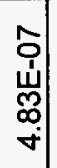 & 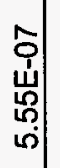 & $\begin{array}{l}\hat{o} \\
\text { 岁 } \\
\stackrel{5}{0} \\
\dot{0}\end{array}$ & 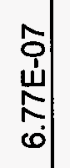 & 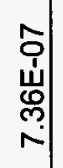 & 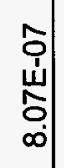 & $\begin{array}{c}\hat{O} \\
⿱ \\
\breve{d} \\
\infty \\
\infty \\
\infty\end{array}$ & 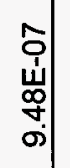 & $\begin{array}{l}0 \\
0 \\
\omega \\
\vdots \\
0 \\
-\end{array}$ & $\begin{array}{l}\stackrel{8}{0} \\
\stackrel{u}{\mathrm{~N}} \\
\stackrel{-}{-}\end{array}$ & 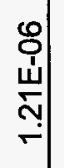 & $\begin{array}{l}\stackrel{8}{0} \\
\stackrel{4}{4} \\
\stackrel{m}{m}\end{array}$ & 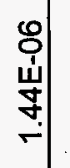 & 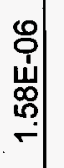 & 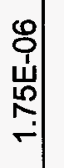 & 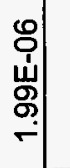 & 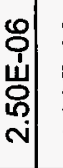 & 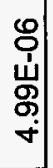 \\
\hline 동 & 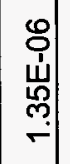 & 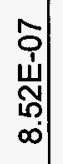 & 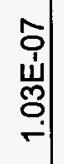 & 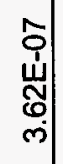 & 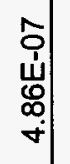 & 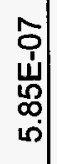 & $\begin{array}{c}\mathbf{5} \\
\stackrel{1}{\mathbf{N}} \\
\underset{0}{0}\end{array}$ & 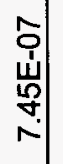 & 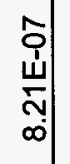 & 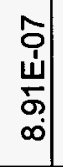 & 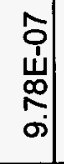 & 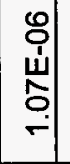 & 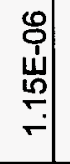 & 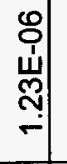 & $\begin{array}{l}0 \\
\vdots \\
\underline{1} \\
0 \\
\stackrel{-}{-}\end{array}$ & 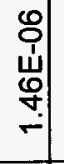 & 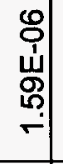 & 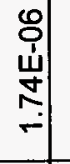 & 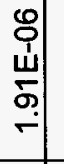 & 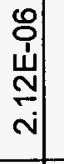 & 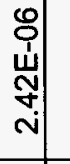 & 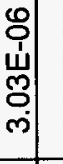 & $\begin{array}{l}\text { ù } \\
\text { ஸ口 } \\
0 \\
0\end{array}$ \\
\hline f & 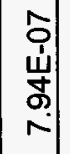 & 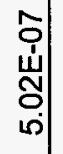 & 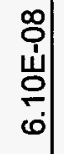 & $\begin{array}{l}\hat{o} \\
\text { 山े } \\
\text { ஸे } \\
\text { ì }\end{array}$ & 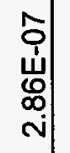 & 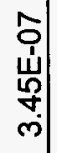 & 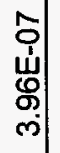 & 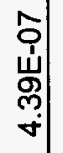 & $\begin{array}{l}\hat{0} \\
\dot{u} \\
\dot{0} \\
\dot{+}\end{array}$ & 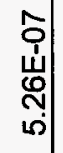 & 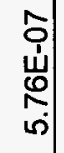 & 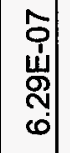 & 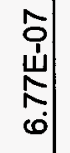 & 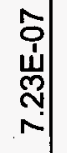 & $\begin{array}{l}\text { 위 } \\
\text { 山े } \\
\text { 이 } \\
\infty\end{array}$ & $\begin{array}{c}\hat{0} \\
\dot{\omega} \\
\stackrel{0}{0} \\
\infty\end{array}$ & 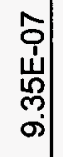 & 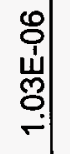 & 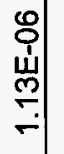 & 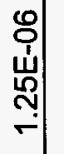 & 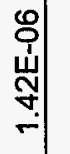 & 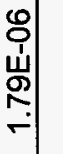 & 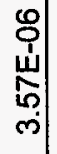 \\
\hline 돜 & 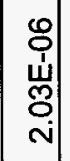 & 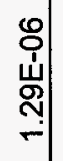 & 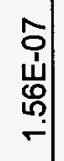 & 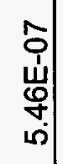 & 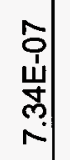 & 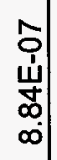 & 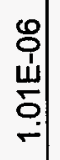 & $\begin{array}{l}\stackrel{0}{0} \\
\stackrel{\leftrightarrow}{4} \\
\stackrel{2}{-}\end{array}$ & 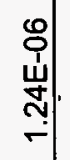 & 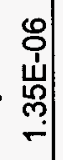 & 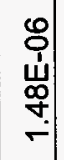 & 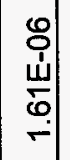 & 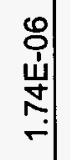 & $\begin{array}{c}\infty \\
0 \\
\dot{\leftrightarrow} \\
0 \\
-\infty\end{array}$ & 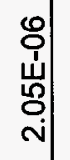 & 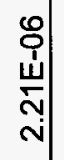 & 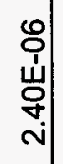 & 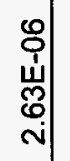 & 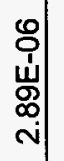 & $\begin{array}{c}\stackrel{8}{0} \\
\stackrel{w}{w} \\
\\
\end{array}$ & 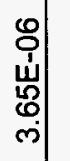 & 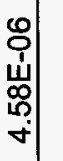 & $\begin{array}{c}\dot{w} \\
\dot{\sigma} \\
\dot{\sigma}\end{array}$ \\
\hline पे & 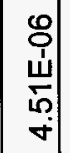 & 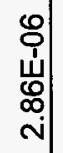 & 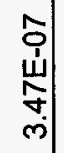 & $\begin{array}{c}0 \\
\vdots \\
w \\
\\
\end{array}$ & 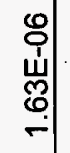 & 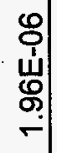 & 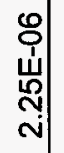 & 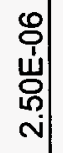 & $\begin{array}{c}\wp \\
0 \\
1 \\
\stackrel{\omega}{0} \\
N\end{array}$ & 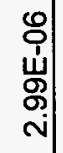 & 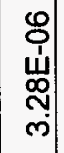 & 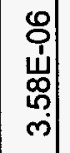 & 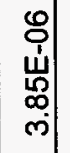 & $\begin{array}{c}o \\
o \\
\dot{1} \\
\tilde{\sigma} \\
\dot{\forall}\end{array}$ & 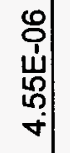 & 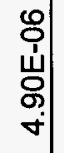 & 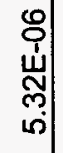 & 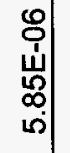 & 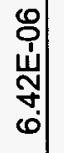 & 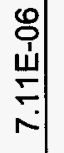 & $\begin{array}{c}\mathbf{8} \\
\dot{1} \\
\ddot{\circ} \\
\dot{\infty}\end{array}$ & 岁 & $\begin{array}{l}\dot{y} \\
\text { ஸे } \\
\text { ஸे }\end{array}$ \\
\hline 兑 & 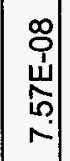 & 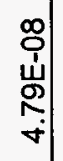 & 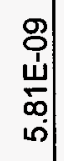 & $\begin{array}{c}\infty \\
0 \\
\dot{u} \\
\tilde{y} \\
i\end{array}$ & 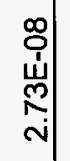 & 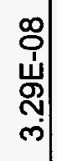 & $\begin{array}{c}\infty \\
0 \\
\dot{u} \\
N \\
\dot{m}\end{array}$ & $\begin{array}{c}\stackrel{\infty}{0} \\
\stackrel{u}{u} \\
\stackrel{0}{\infty} \\
\dot{\forall}\end{array}$ & $\begin{array}{l}\infty \\
0 \\
\dot{w} \\
\check{0} \\
\dot{+}\end{array}$ & 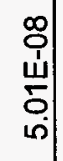 & 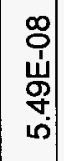 & 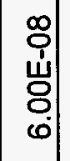 & $\left|\begin{array}{c}0 \\
0 \\
1 \\
u \\
0 \\
0 \\
0\end{array}\right|$ & 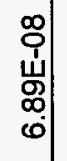 & $\begin{array}{c}\infty \\
0 \\
⿱ \\
\mathbb{N} \\
\vdots \\
\end{array}$ & 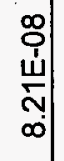 & 怘 & 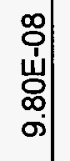 & 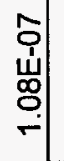 & 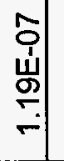 & 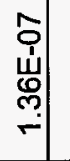 & 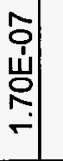 & 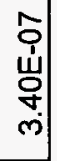 \\
\hline$\frac{0}{0}$ & 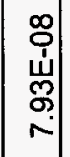 & 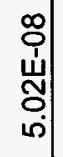 & 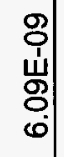 & 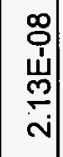 & 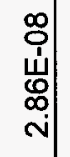 & 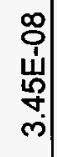 & 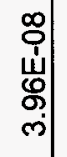 & 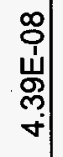 & 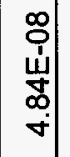 & 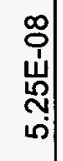 & 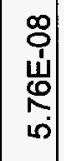 & 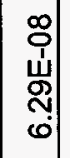 & $\begin{array}{l}\infty \\
0 \\
w \\
\hat{\uparrow} \\
0\end{array}$ & $\begin{array}{l}\infty \\
\vdots \\
\stackrel{1}{N} \\
\text { N̦}\end{array}$ & 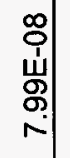 & 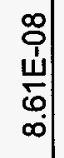 & 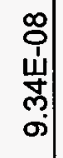 & $\begin{array}{c}\text { ó } \\
\text { 山్ } \\
0 \\
0\end{array}$ & 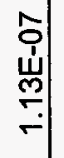 & 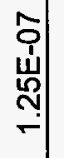 & 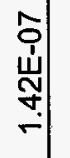 & 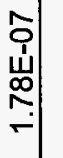 & 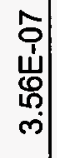 \\
\hline ن & $\begin{array}{c}\overline{\dot{u}} \\
\stackrel{\bar{m}}{\dot{m}}\end{array}$ & 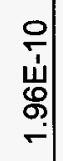 & 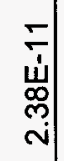 & 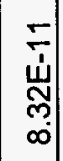 & 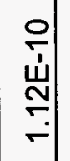 & 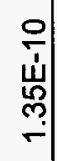 & $\begin{array}{l}\text { 움 } \\
\dot{4} \\
\dot{+} \\
-5\end{array}$ & $\begin{array}{l}\text { 울 } \\
\stackrel{\omega}{i}\end{array}$ & $\mid \begin{array}{c}\text { 어 } \\
\text { 山े } \\
\stackrel{\circ}{-}\end{array}$ & 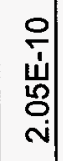 & 은 & 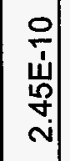 & 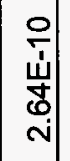 & 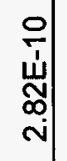 & 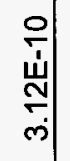 & 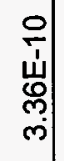 & 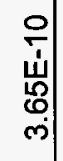 & $\begin{array}{l}\frac{0}{4} \\
\frac{1}{\delta} \\
\dot{\gamma}\end{array}$ & $\begin{array}{l}\text { uे } \\
\text { Uे } \\
\dot{v}\end{array}$ & $\begin{array}{l}\vec{u} \\
\mathbf{\infty} \\
\infty \\
\dot{\forall}\end{array}$ & 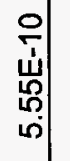 & 움 & 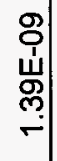 \\
\hline 5 & 웜 & 웜 & $\begin{array}{l}\stackrel{\tau}{\dot{u}} \\
\stackrel{+}{-}\end{array}$ & 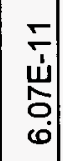 & 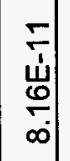 & 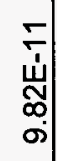 & $\begin{array}{l}\stackrel{\circ}{\cdot} \\
\stackrel{m}{\sigma} \\
\stackrel{-}{-}\end{array}$ & 윔 & 어 & 울 & 움 & $\begin{array}{l}\text { 음 } \\
\text { 山े } \\
\stackrel{2}{\circ}\end{array}$ & 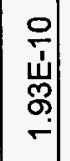 & 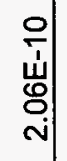 & 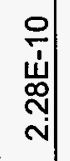 & 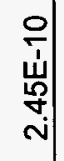 & $\begin{array}{l}\text { 윰 } \\
\text { 岁 } \\
\text { ํ. }\end{array}$ & 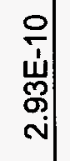 & $\begin{array}{l}\text { 윕. } \\
\text { ஸे } \\
\text { m. }\end{array}$ & 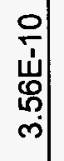 & $\begin{array}{l}\text { 은 } \\
\text { 山े } \\
\stackrel{0}{0} \\
\dot{\nabla}\end{array}$ & 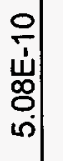 & 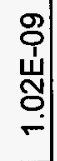 \\
\hline & 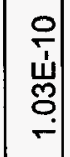 & 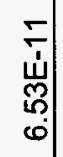 & 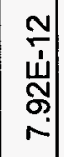 & 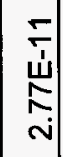 & 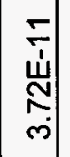 & 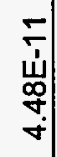 & 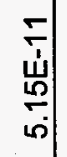 & $\begin{array}{c}\underset{\dot{w}}{\tilde{\omega}} \\
\underset{\omega}{\dot{0}}\end{array}$ & 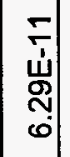 & 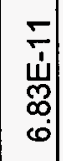 & 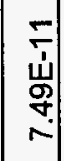 & 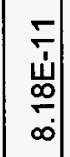 & $\begin{array}{c}\mp \\
\\
\text { ய் } \\
\infty \\
\infty\end{array}$ & \begin{tabular}{c}
\multicolumn{5}{c}{} \\
山े \\
ఫे \\
के
\end{tabular} & 일. & $\begin{array}{c}\stackrel{ }{\grave{d}} \\
\stackrel{\mathrm{w}}{\mathrm{N}} \\
\stackrel{-}{-}\end{array}$ & 울 & 은 & $\begin{array}{c}\text { 웜 } \\
\stackrel{4}{*} \\
\stackrel{5}{\circ}\end{array}$ & 울 & 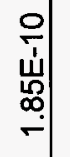 & $\begin{array}{l}\text { 울 } \\
\stackrel{\sim}{N} \\
\stackrel{N}{N}\end{array}$ & $\begin{array}{l}\text { 움 } \\
\text { س̦ } \\
\dot{+}\end{array}$ \\
\hline & 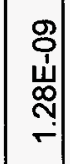 & $\underset{\infty}{\stackrel{ }{\dot{\omega}}}$ & 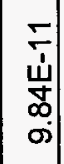 & 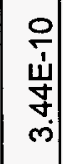 & 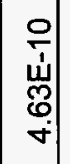 & $\begin{array}{l}\text { 운 } \\
\dot{\dot{b}} \\
\stackrel{5}{0} \\
\omega\end{array}$ & 웋 & 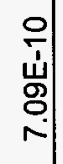 & 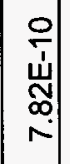 & 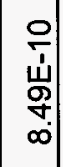 & $\begin{array}{l}\text { 윔 } \\
\text { w. } \\
\stackrel{m}{m} \\
\sigma^{2}\end{array}$ & 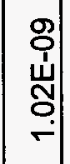 & 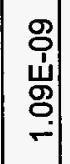 & 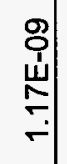 & 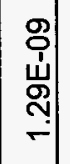 & $\begin{array}{c}\stackrel{0}{0} \\
\dot{u} \\
\text { ⿹े } \\
\stackrel{9}{-}\end{array}$ & 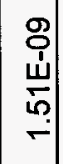 & 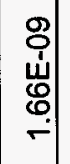 & 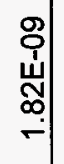 & 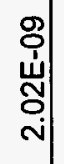 & 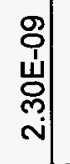 & 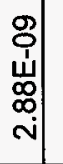 & $\begin{array}{l}0 \\
\text { 足 } \\
\stackrel{0}{0}\end{array}$ \\
\hline & $\begin{array}{l}\frac{c}{\mathbb{J}} \\
\sum^{\mathbb{d}}\end{array}$ & 잉 & $\dot{\Sigma}$ & î & 인 & ㅇํㅇ & 今̊ & 怘 & : & $\mid$ & $\mid \begin{array}{l}0 \\
0\end{array}$ & 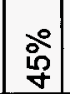 & $\begin{array}{l}\text { : } \\
0 \\
0\end{array}$ & 艿 & $\mid \begin{array}{c}80 \\
8 \\
0\end{array}$ & 突 & Q & 今̊ํํ & ○े| & 总 & ৪̊ㅇ & 吕 & L \\
\hline
\end{tabular}




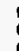

彥

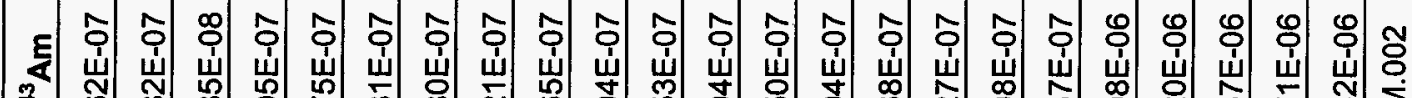

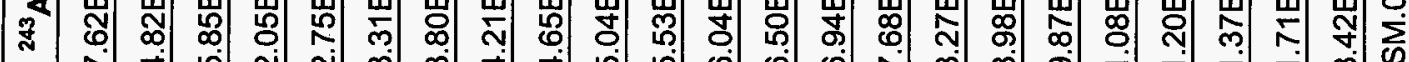

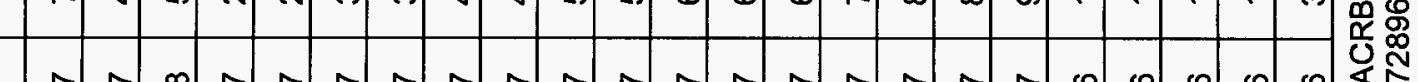

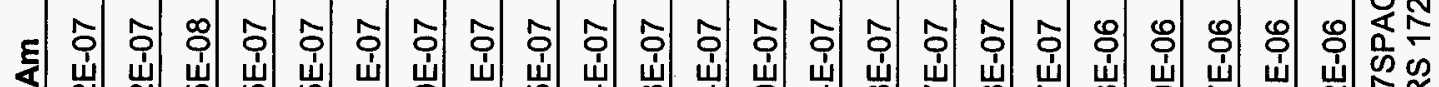
₹ స

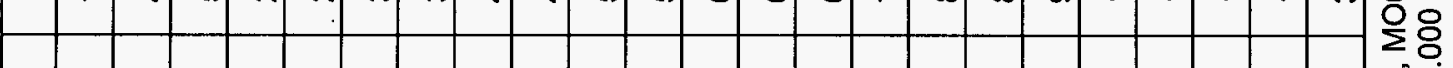

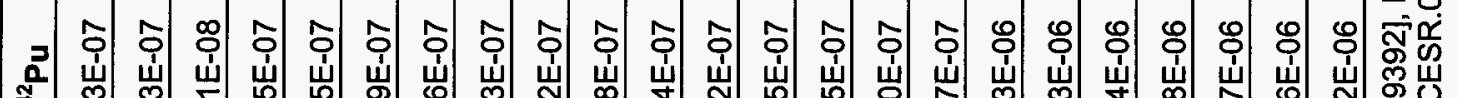

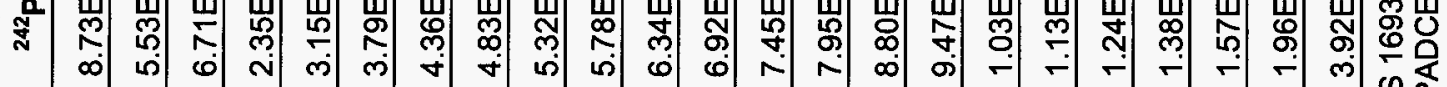

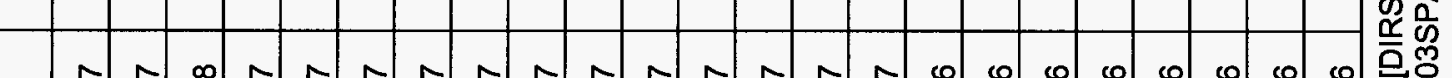

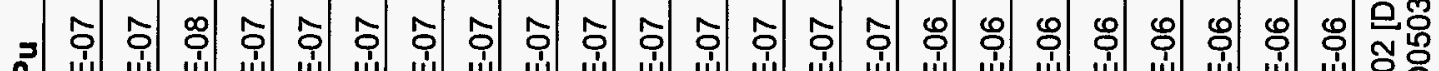

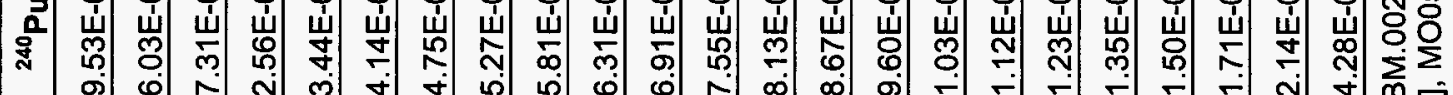
の

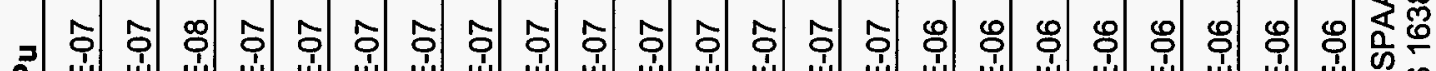

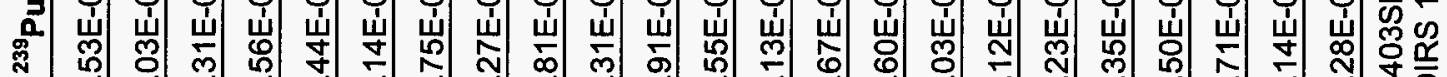
\%

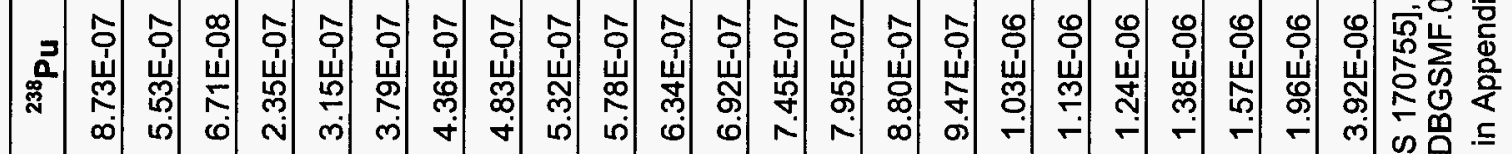

ஜ

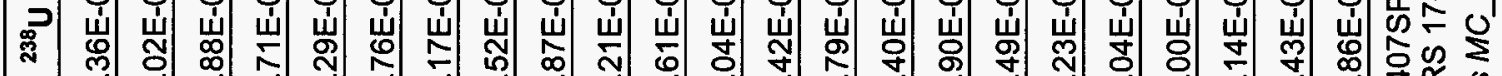

$\frac{\text { ह }}{\frac{E}{D}}$

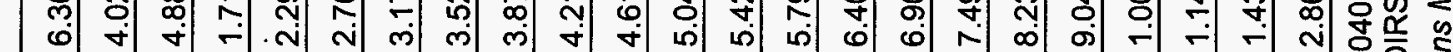
*

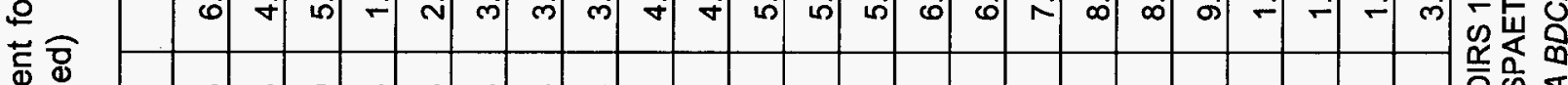

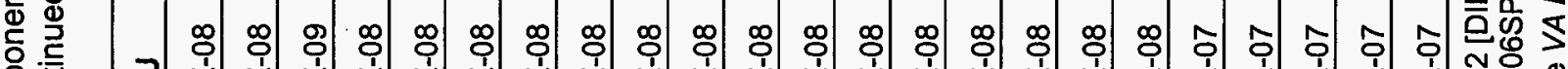

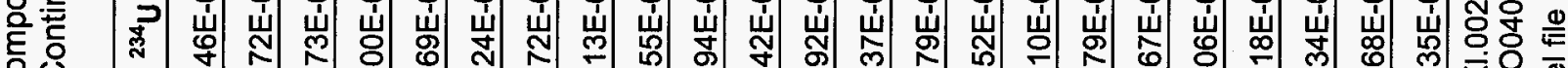

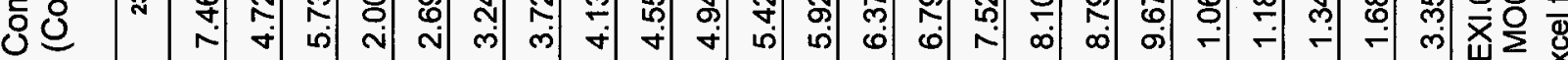

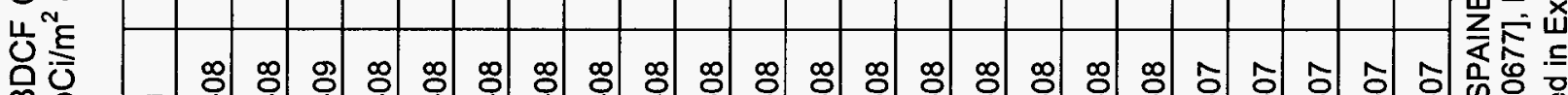

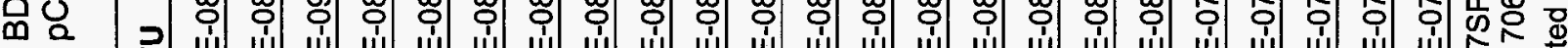

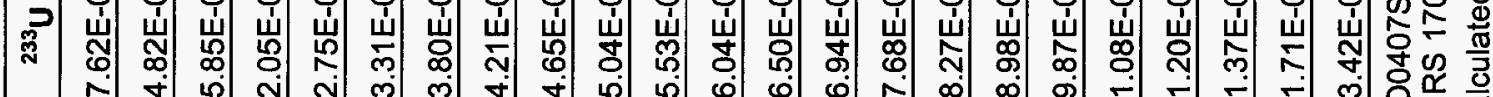

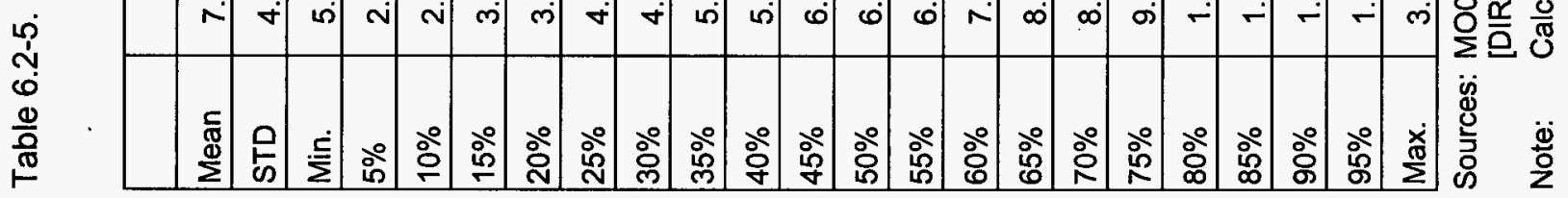




\begin{tabular}{|c|c|c|c|c|c|c|c|c|c|c|c|c|c|c|c|c|c|c|c|c|c|c|c|}
\hline$=$ & 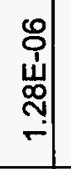 & 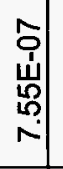 & 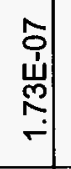 & 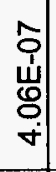 & $\begin{array}{l}\text { 잉 } \\
\text { 웜 }\end{array}$ & 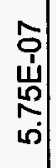 & 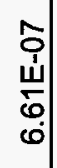 & 品 & 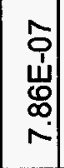 & 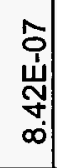 & 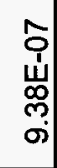 & 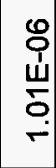 & 岁 & 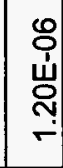 & 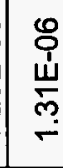 & 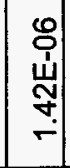 & 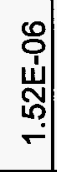 & 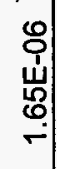 & 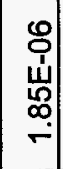 & 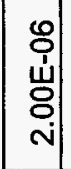 & 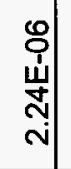 & $\begin{array}{l}0 \\
0 \\
\text { W } \\
\text { N } \\
\text { N }\end{array}$ & 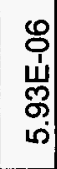 \\
\hline$\stackrel{0}{0}$ & 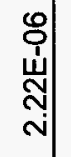 & $\begin{array}{l}\stackrel{8}{o} \\
\stackrel{\leftrightarrow}{\omega} \\
\stackrel{m}{-}\end{array}$ & 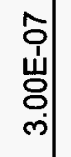 & 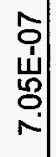 & $\begin{array}{c}\text { 엄 } \\
\text { 山్ } \\
\text { } \\
\infty\end{array}$ & 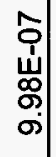 & 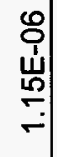 & 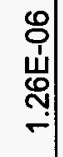 & 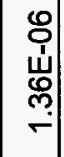 & 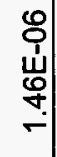 & $\begin{array}{l}\text { Oे } \\
\text { 山్ } \\
00 \\
-\end{array}$ & $\begin{array}{l}\mathscr{8} \\
\stackrel{1}{1} \\
\text { 足 } \\
\stackrel{2}{r}\end{array}$ & 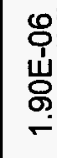 & 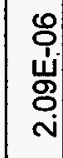 & 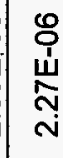 & 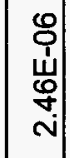 & 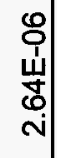 & 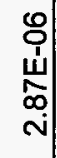 & 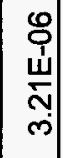 & \begin{tabular}{l}
0 \\
0 \\
$\dot{\omega}$ \\
\multirow{J}{y}{} \\
ஸे
\end{tabular} & $\begin{array}{c}\mathscr{O} \\
\dot{1} \\
\ddot{ळ} \\
\infty \\
m\end{array}$ & $\begin{array}{l}\mathscr{O} \\
\dot{1} \\
\stackrel{N}{N} \\
\dot{v}\end{array}$ & 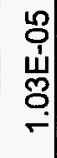 \\
\hline 동 & $\begin{array}{l}\text { 山े. } \\
\stackrel{0}{0}\end{array}$ & 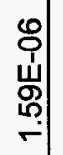 & 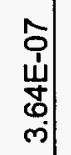 & 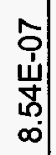 & 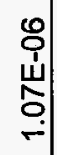 & $\begin{array}{c}\stackrel{8}{0} \\
\omega \\
\stackrel{\omega}{\omega} \\
\end{array}$ & $\begin{array}{c}0 \\
\stackrel{0}{0} \\
\stackrel{\leftrightarrow}{9} \\
\stackrel{9}{0}\end{array}$ & 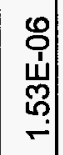 & 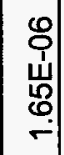 & 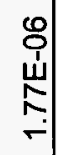 & 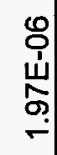 & 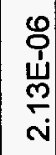 & 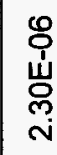 & 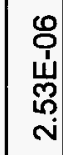 & 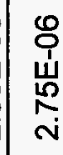 & 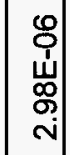 & 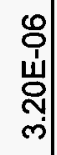 & 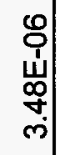 & 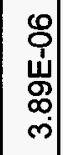 & דั & $\begin{array}{l}\mathscr{O} \\
\stackrel{+}{ } \\
\ddot{T} \\
\dot{\nabla}\end{array}$ & 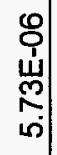 & 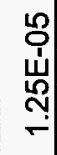 \\
\hline$\vec{f}$ & 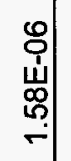 & $\begin{array}{c}\hat{0} \\
\stackrel{4}{4} \\
\hat{j} \\
o\end{array}$ & $\begin{array}{c}\hat{o} \\
\dot{\jmath} \\
\dot{y} \\
\grave{v}\end{array}$ & 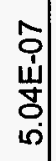 & 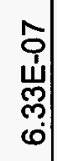 & 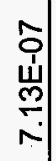 & 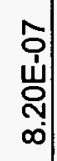 & $\begin{array}{c}\hat{o} \\
\text { ù } \\
\text { o. } \\
\text { o. }\end{array}$ & 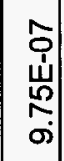 & 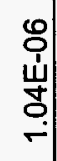 & 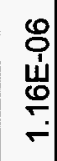 & 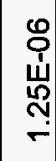 & 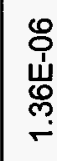 & 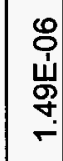 & 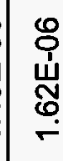 & 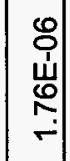 & \begin{tabular}{l} 
ơ \\
W్ \\
$\stackrel{0}{0}$ \\
\hdashline
\end{tabular} & 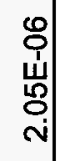 & 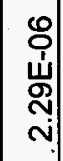 & | & 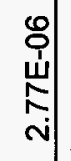 & 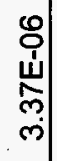 & \\
\hline$\underset{N}{\mathbf{f}}$ & $\begin{array}{l}0 \\
0 \\
⿱ 1 \\
\dddot{8} \\
\dot{\forall}\end{array}$ & 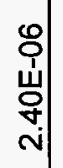 & $\begin{array}{l}0 \\
\text { w్ } \\
\text { ơ } \\
\text { ம் }\end{array}$ & 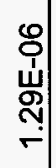 & 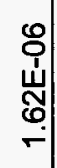 & 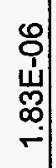 & $\begin{array}{l}\text { o } \\
\text { 山े } \\
\text { 휘 } \\
\text { i }\end{array}$ & 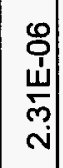 & 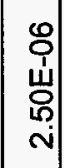 & 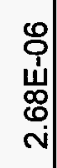 & 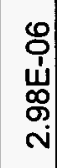 & $\begin{array}{l}\stackrel{8}{0} \\
\dot{3} \\
\\
\text { m. }\end{array}$ & 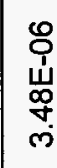 & 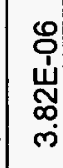 & $\frac{\dot{m}}{\dot{v}}$ & $\begin{array}{c}\text { 山े } \\
\text { مீ } \\
\dot{\nabla}\end{array}$ & 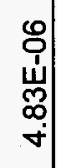 & 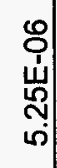 & 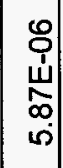 & 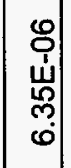 & 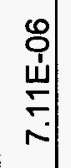 & $\begin{array}{l}\text { 崩 } \\
0 \\
\infty \\
0\end{array}$ & $\begin{array}{l}\text { 岁 } \\
\infty \\
\stackrel{\infty}{\circ}\end{array}$ \\
\hline 婇 & 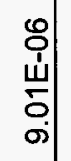 & 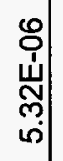 & ড় & $\begin{array}{l}\stackrel{u}{ } \\
\stackrel{0}{0} \\
\stackrel{0}{*}\end{array}$ & 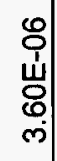 & 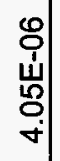 & 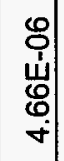 & 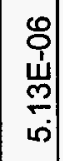 & 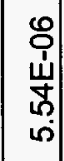 & 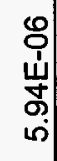 & $\begin{array}{l}\dot{\omega} \\
\stackrel{5}{6} \\
\dot{\phi}\end{array}$ & 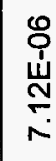 & $\begin{array}{l}\text { O } \\
\stackrel{1}{4} \\
\stackrel{N}{N} \\
\end{array}$ & $\begin{array}{c}0 \\
⿱ \\
w \\
w \\
o \\
\infty \\
\infty\end{array}$ & 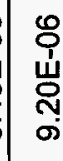 & \begin{tabular}{|c|}
$\dot{u}$ \\
$\mathbf{0}$ \\
0
\end{tabular} & 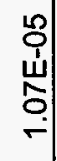 & 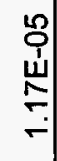 & 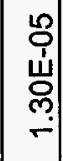 & 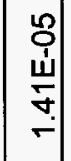 & 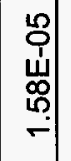 & 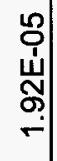 & 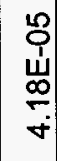 \\
\hline$\underset{\mathbb{N}}{\mathbb{N}}$ & 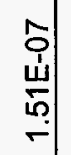 & 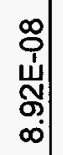 & \begin{tabular}{|c|}
$\dot{\Psi}$ \\
$\dot{\mathbf{v}}$
\end{tabular} & 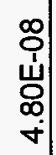 & 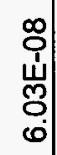 & 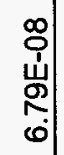 & 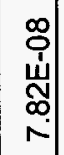 & 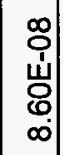 & $\mid \begin{array}{c}0 \\
0 \\
\text { 山్ } \\
\stackrel{2}{2} \\
\sigma\end{array}$ & 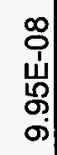 & 亗 & 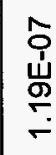 & 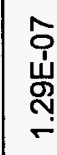 & 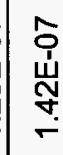 & 客 & $\begin{array}{l}\dot{\omega} \\
\sigma\end{array}$ & 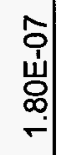 & 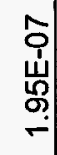 & 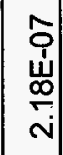 & 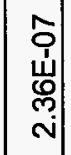 & 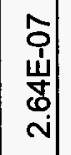 & 岕 & 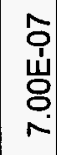 \\
\hline a & 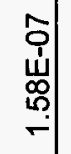 & 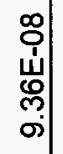 & 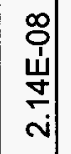 & 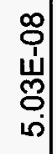 & 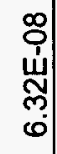 & 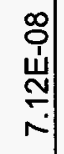 & 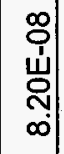 & 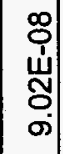 & 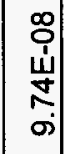 & 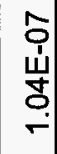 & $\begin{array}{l}\hat{0} \\
\text { ư } \\
\stackrel{0}{0}\end{array}$ & 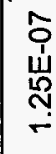 & $\begin{array}{l}\text { 울 } \\
\text { 山ै } \\
\text { ले } \\
\text {. }\end{array}$ & 岁 & 山् & 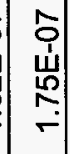 & 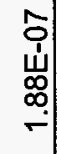 & 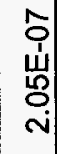 & $\begin{array}{l}\text { W్ } \\
\text { N̦}\end{array}$ & | & $\dot{u}$ & 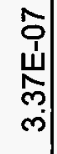 & $\stackrel{\substack{\underline{L}\\
}}{n}$ \\
\hline s. & 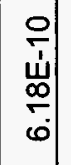 & 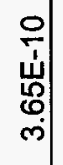 & 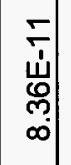 & 离 & 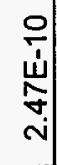 & 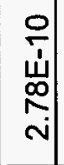 & 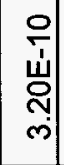 & 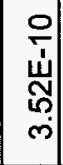 & $\begin{array}{l}\text { 움 } \\
\dot{山} \\
0 \\
0 \\
\dot{n}\end{array}$ & 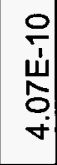 & 岕 & $\begin{array}{l}\text { 岁 } \\
\text { ळ } \\
\dot{\sim}\end{array}$ & $\begin{array}{l}\text { 岁 } \\
\text { ڤn }\end{array}$ & 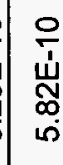 & 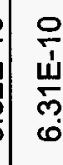 & 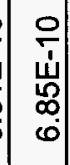 & 岁 & 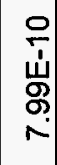 & $\mid \begin{array}{c}0 \\
\dot{\omega} \\
\dot{\sigma} \\
\infty \\
\infty\end{array}$ & ?. & $\therefore$ & 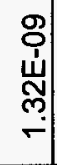 & $\stackrel{u^{\prime}}{\infty}$ \\
\hline ஜี & 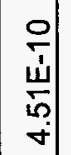 & 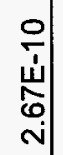 & 它 & 윔 & $\begin{array}{l}\text { 윔 } \\
\dot{u} \\
\text { ㅁ } \\
\dot{\circ}\end{array}$ & 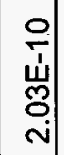 & 岕 & 의 & $\begin{array}{l}0 \\
\dot{\sim} \\
\hat{N} \\
\hat{N}\end{array}$ & $\begin{array}{l}\stackrel{ }{\overline{\dot{w}}} \\
\text { ธे }\end{array}$ & 힘 & $\begin{array}{l}\overline{1} \\
\tilde{L} \\
\tilde{\omega}\end{array}$ & $\begin{array}{l}\overline{\text { uे }} \\
\otimes \\
\text { ले }\end{array}$ & 出 & 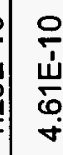 & $\mid \begin{array}{c}\text { 윔 } \\
\text { 山े } \\
\text { 이 }\end{array}$ & 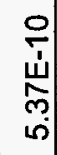 & 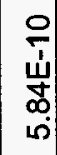 & 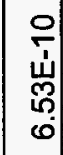 & in & ய் & 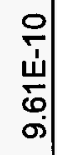 & $\underline{u}$ \\
\hline 1 & 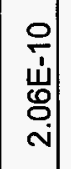 & 윌 & 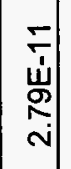 & 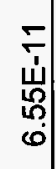 & 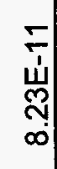 & テَ & 읙 & 웜 & $\begin{array}{l}\text { 울 } \\
\stackrel{4}{N} \\
\end{array}$ & 움 & 울 & 㟧 & 5 & 安 & $\frac{\bar{u}}{\stackrel{\sim}{\Delta}}$ & $\underset{\substack{\mathrm{w} \\
\mathrm{N}}}{ }$ & 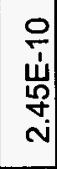 & 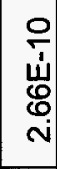 & دأ & 岕 & $\begin{array}{l}\text { 윟 } \\
\text { யे } \\
\overline{6} \\
\dot{m}\end{array}$ & $\begin{array}{l}\text { 岁 } \\
\text { 户े } \\
\dot{v}\end{array}$ & ய் \\
\hline : & 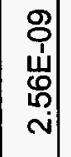 & 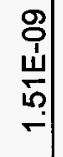 & 음 & $\begin{array}{c}\text { 움 } \\
\dot{山} \\
\stackrel{m}{\infty} \\
\infty\end{array}$ & 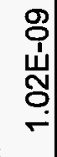 & 总 & ơ & 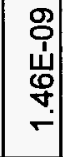 & 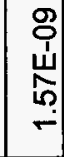 & 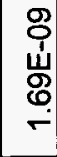 & $\begin{array}{l}\text { 㞻 } \\
\text { జू } \\
\text { o. }\end{array}$ & N & 岁 & 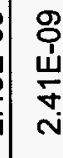 & 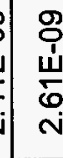 & 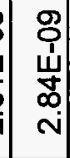 & 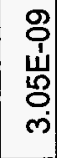 & Щّ & 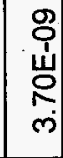 & $\begin{array}{l}\text { 山े } \\
\text { ல் }\end{array}$ & 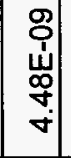 & 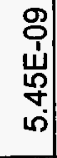 & 㟧 \\
\hline & 跑 & $\begin{array}{l}0 \\
\text { 号 }\end{array}$ & 这 & î́. & 웅 & ㅇํㄴ & iิ & 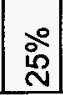 & கి & m & 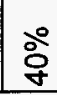 & & 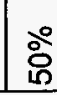 & 茴 & 8 & 80 & $R$ & in & $\infty$ & 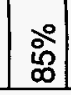 & 亏ें। & 18 & \\
\hline
\end{tabular}


造

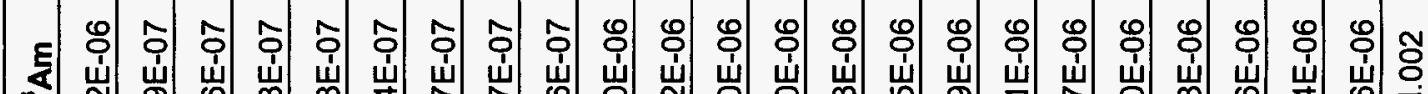

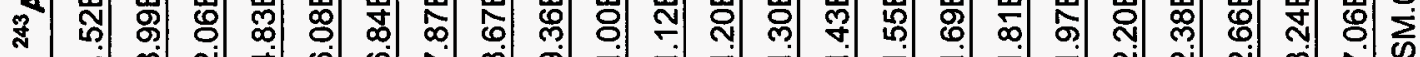

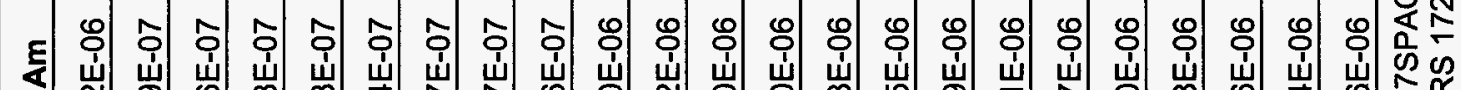

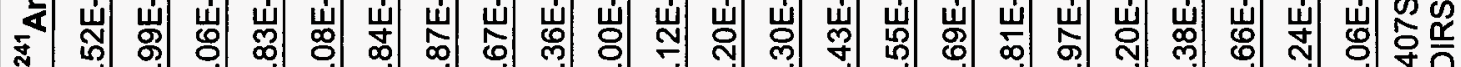

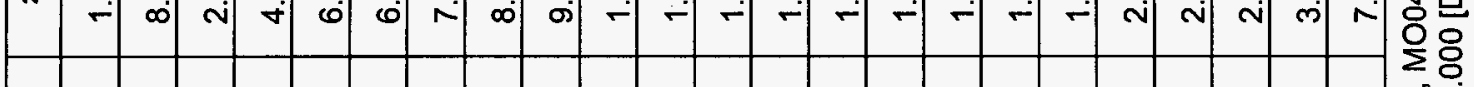

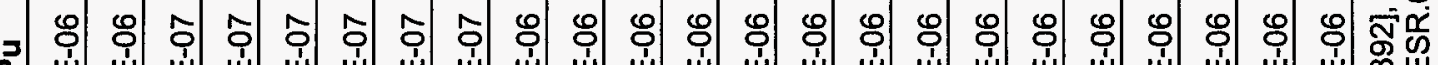

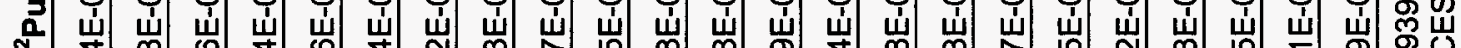
ป స ₹

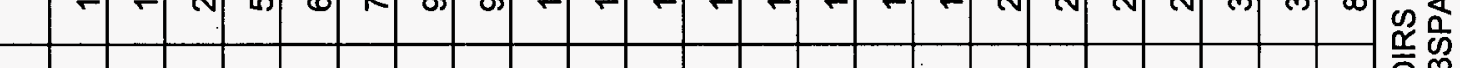
ᄀ

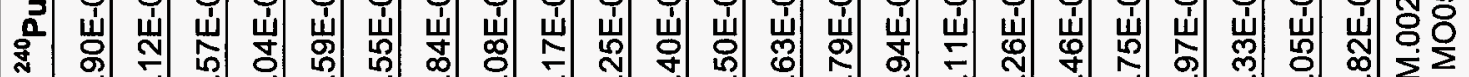

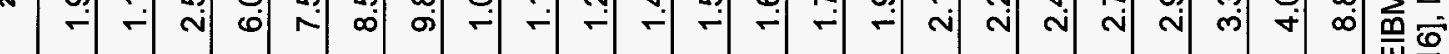
\&

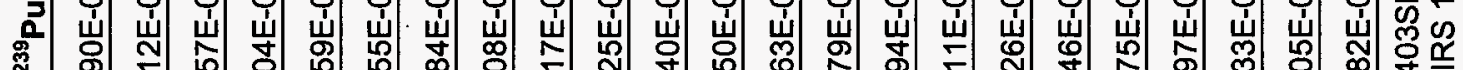
\%

$\frac{0}{\frac{1}{0}}$

\&

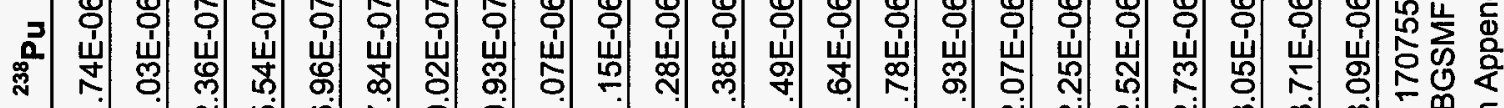
高

㟒

妾

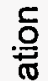

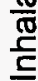

ह

$\stackrel{\bullet}{\leftarrow}$

ㅎํㅇ

పे

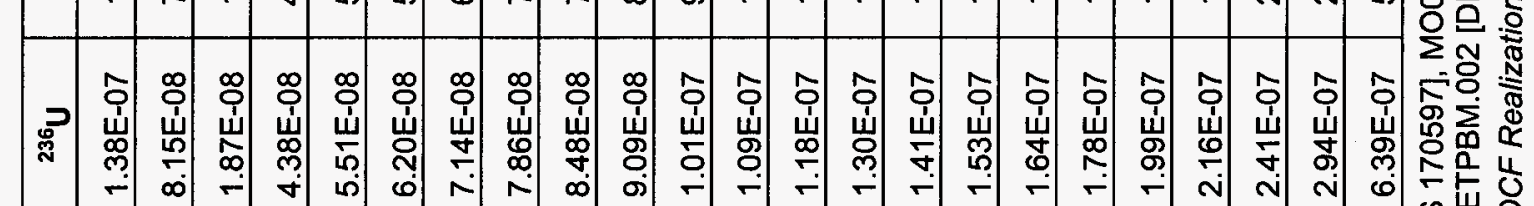

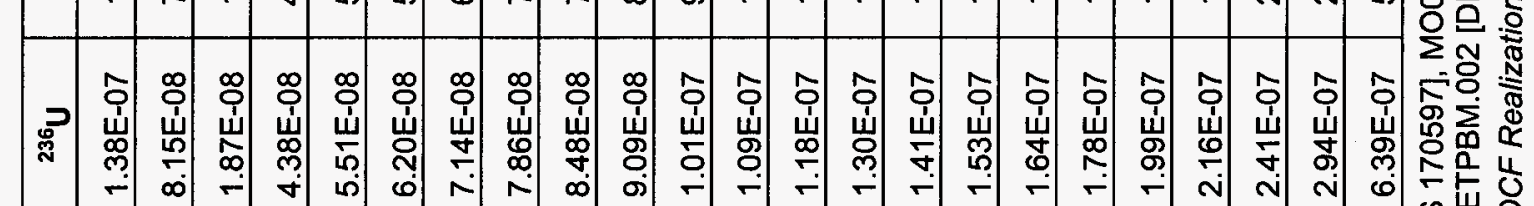

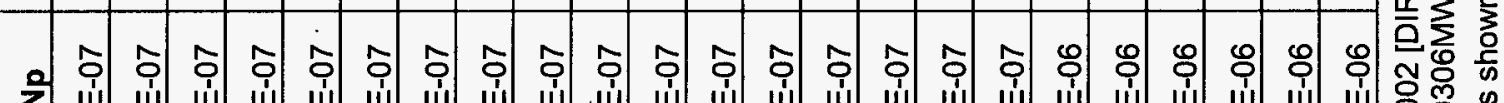

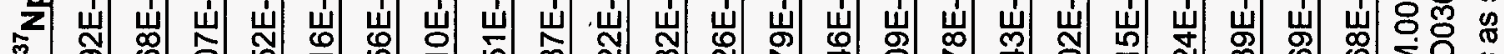

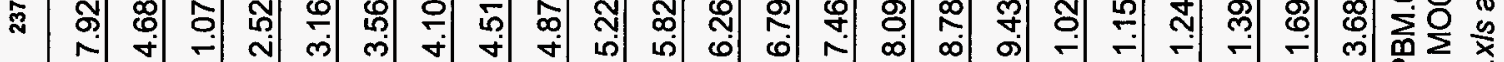

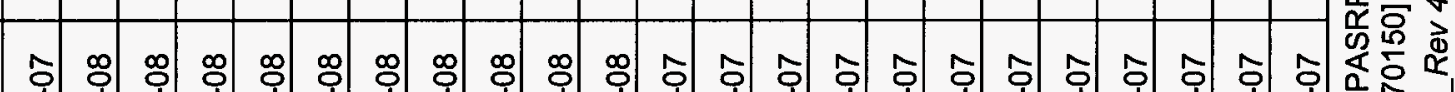

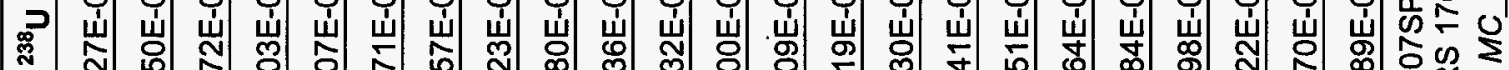
艺君 윙 : 至

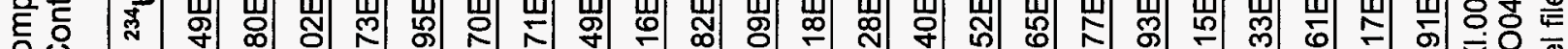

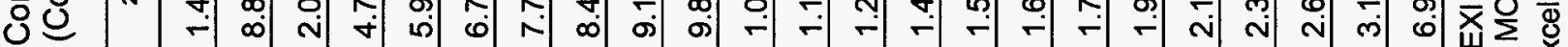

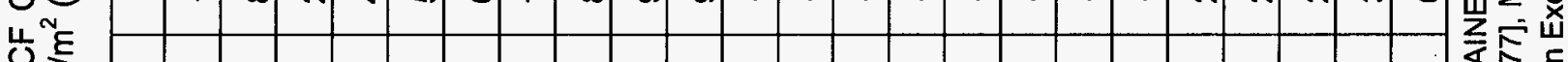

施

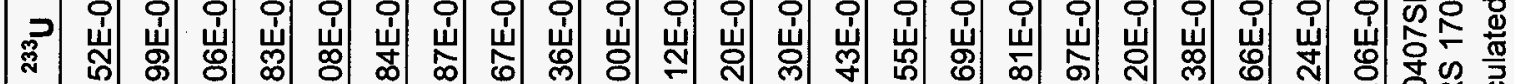

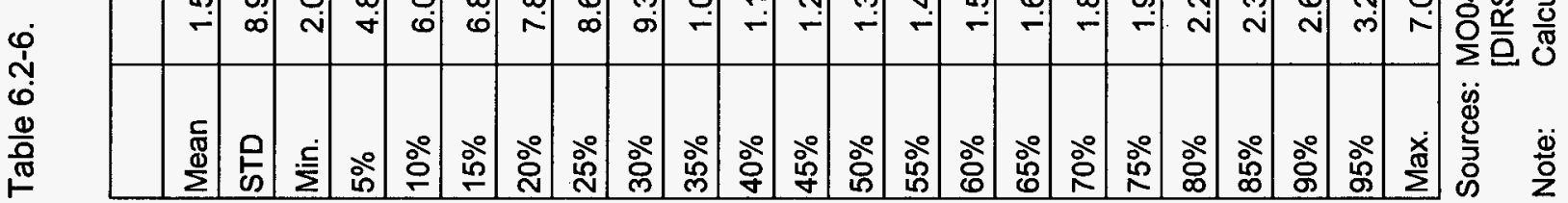


The mean values of the external-ingestion-radon BDCF components for the present-day climate calculated using the ICRP 30 and ICRP 72 dosimetries are compared in Figure 6.2-1.

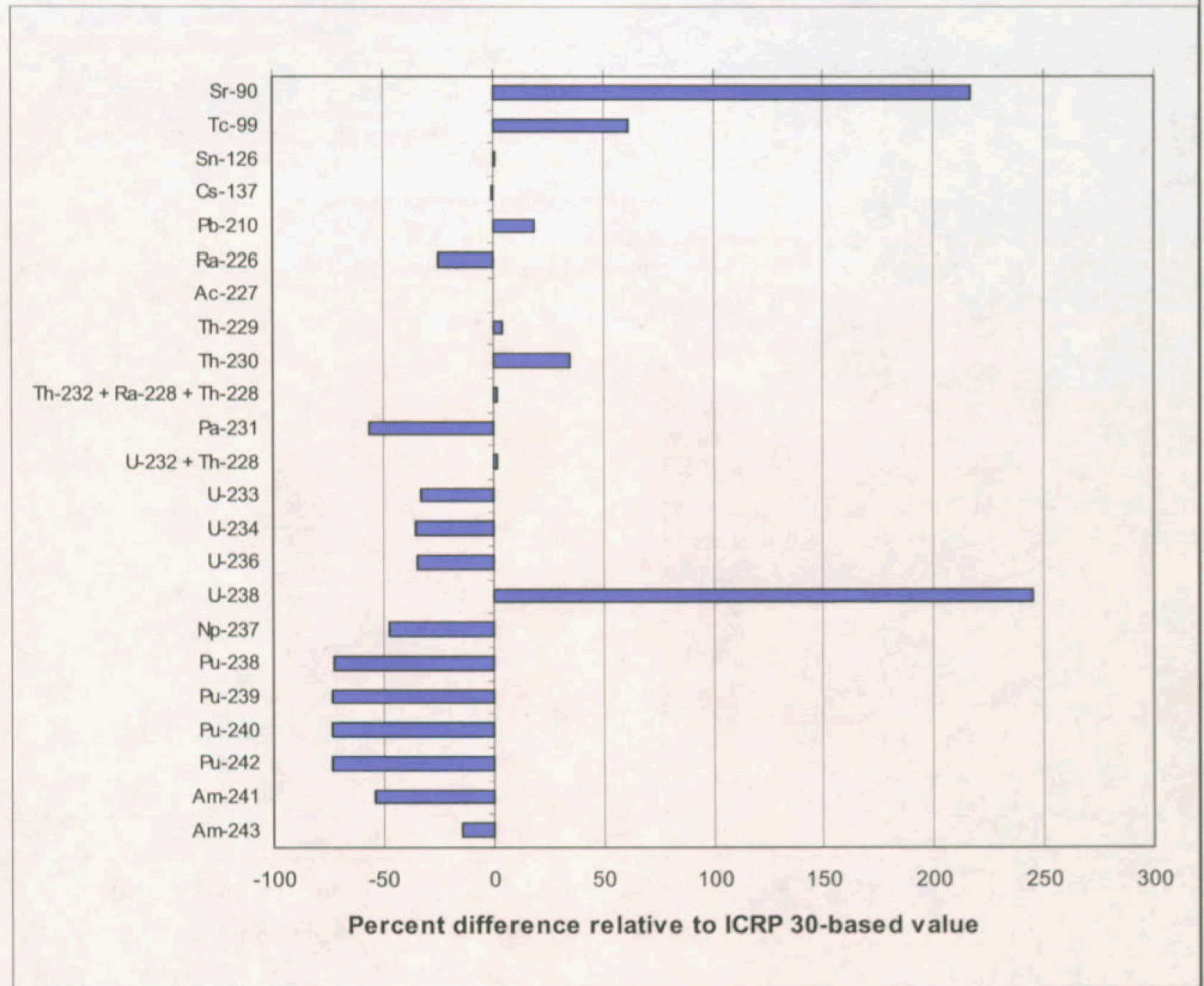

Source: The values were calculated in Excel file VA BDCF Realizations MC_Rev 4.x/s. See Appendix B for details of calculations.

Figure 6.2-1. Comparison of ICRP 30- and ICRP 72-Based BDCF Components for External Exposure, Ingestion, and Inhalation of Radon Decay Products for the Present-Day Climate

For the external-ingestion-radon component (Figure 6.2-1), the changes result from the combined effect of ingestion, external exposure, and radon inhalation dose coefficient updates. For most actinides, this BDCF component has a lower value when ICRP 72 dose coefficients are used. A notable exception is ${ }^{238} \mathrm{U}$, which has much higher dose coefficient for external exposure for one of its short-lived decay products. The higher ICRP 72 external-ingestion-radon BDCF component for ${ }^{99} \mathrm{Tc}$ is due to increase in the ingestion dose coefficient for this radionuclide; the higher value for ${ }^{90} \mathrm{Sr}$ results from the higher dose coefficients for external exposure for ${ }^{90} \mathrm{Sr}$ and its decay product, ${ }^{90} \mathrm{Y}$. 
The mean values of the inhalation BDCF components for the present-day climate calculated using the ICRP 30 and ICRP 72 dosimetries are compared in Figure 6.2-2.

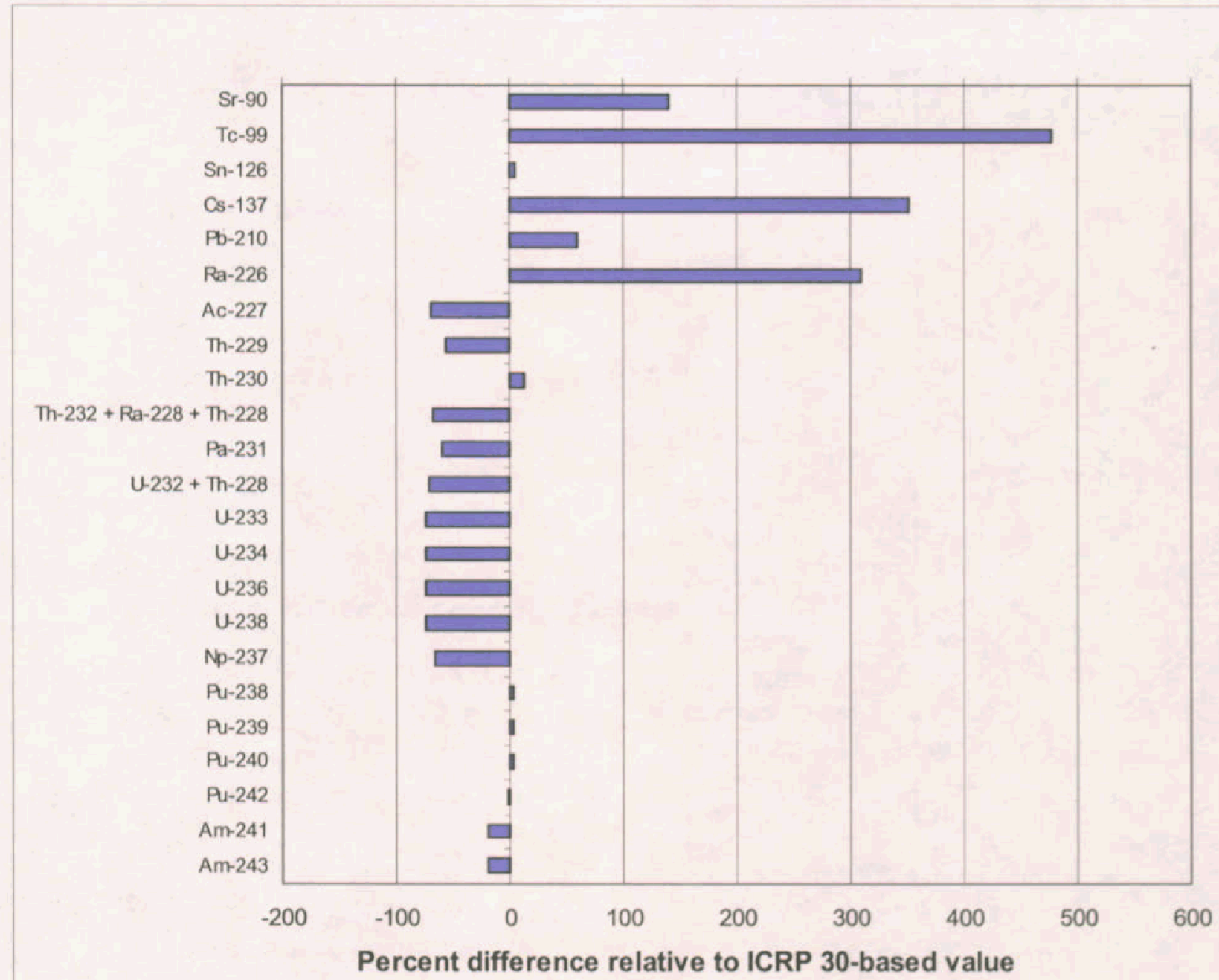

Source: The values were calculated in Excel file VA BDCF Realizations MC_Rev 4.x/s. See Appendix B for details of calculations.

Figure 6.2-2. Comparison of ICRP 30- and ICRP 72-Based BDCF Components for Inhalation of Particulate Matter for the Present-Day Climate

The differences in the inhalation BDCF components (Figure 6.2-2) result only from the update in the inhalation dose coefficients (see BSC 2005 [DIRS 172827], Figure 6-13) from ICRP 30based values to ICRP 72 values. For the inhalation components, the BDCFs for the actinides are generally lower when the ICRP 72 dosimetric methods are used. For the lighter radionuclides, inhalation BDCF components are greater when ICRP 72 dosimetry is used, up to almost 5 times for ${ }^{99} \mathrm{Tc}$.

Correlations among BDCF components for individual radionuclides and among BDCF components or various radionuclides have been established. In general, the BDCF components 
for the volcanic ash scenario can be correlated because some of the input parameters used to calculate these components may be the same. Correlations exist between the BDCF components for a given radionuclide and between the BDCF components for different radionuclides. The correlation coefficients for the BDCF components for individual radionuclides are listed in Tables 6.2-7 and 6.2-8 for the ICRP 30- and ICRP 72-based values, respectively. There is no correlation or a very poor correlation between the external-ingestion-radon BDCF component and any of the inhalation components because these components are related to different exposure pathways and share only a few input parameters. A stronger correlation exists only between the inhalation BDCF components, the long-term and the short-term, because they depend on the same set of parameters describing behavior of the receptor and the receptor environments.

Table 6.2-7. Correlation Coefficients for the Volcanic Ash Scenario ICRP 30-Based BDCF Components

\begin{tabular}{|l|c|c|c|}
\hline Radionuclide & $\begin{array}{c}\text { External-Ingestion-Radon and } \\
\text { Short-term Inhalation }\end{array}$ & $\begin{array}{c}\text { Short-term Inhalation and } \\
\text { Long-term Inhalation }\end{array}$ & $\begin{array}{c}\text { External-Ingestion-Radon and } \\
\text { Long-term Inhalation }\end{array}$ \\
\hline${ }^{90} \mathrm{Sr}$ & 0 & 0.609 & 0 \\
\hline${ }^{99} \mathrm{Tc}$ & 0 & 0.609 & 0 \\
\hline${ }^{126} \mathrm{Sn}$ & 0.112 & 0.609 & 0.155 \\
\hline${ }^{137} \mathrm{Cs}$ & 0.101 & 0.609 & 0.139 \\
\hline${ }^{210} \mathrm{~Pb}$ & 0 & 0.609 & 0 \\
\hline${ }^{226} \mathrm{Ra}$ & 0 & 0.609 & 0 \\
\hline${ }^{227} \mathrm{Ac}$ & 0 & 0.609 & 0 \\
\hline${ }^{229} \mathrm{Th}$ & 0 & 0.609 & 0 \\
\hline${ }^{230} \mathrm{Th}$ & 0 & 0.609 & 0 \\
\hline${ }^{232} \mathrm{Th}$ & 0 & 0.609 & 0.101 \\
\hline${ }^{231} \mathrm{~Pa}$ & 0 & 0.609 & 0 \\
\hline${ }^{232} \mathrm{U}$ & 0 & 0.609 & 0 \\
\hline${ }^{233} \mathrm{U}$ & 0 & 0.609 & 0 \\
\hline${ }^{234} \mathrm{U}$ & 0 & 0.609 & 0 \\
\hline${ }^{236} \mathrm{U}$ & 0 & 0.609 & 0 \\
\hline${ }^{238} \mathrm{U}$ & 0 & 0.609 & 0 \\
\hline${ }^{237} \mathrm{~Np}$ & 0 & 0.609 & 0 \\
\hline${ }^{238} \mathrm{Pu}$ & 0 & 0.609 & 0 \\
\hline${ }^{239} \mathrm{Pu}$ & 0 & 0.609 & 0 \\
\hline${ }^{240} \mathrm{Pu}$ & 0 & 0.609 & 0.609 \\
\hline${ }^{242} \mathrm{Pu}$ & 0 & 0.609 & 0 \\
\hline${ }^{241} \mathrm{Am}$ & 0 & 0.609 & 0 \\
\hline & 0 & 0 & 0 \\
\hline
\end{tabular}

Sources: MO0407SPAINEXI.002 [DIRS 170597], MO0407SPASRPBM.002 [DIRS 170755], MO0403SPAAEIBM.002 [DIRS 169392]; MO0407SPACRBSM.002 [DIRS 170677], MO0406SPAETPBM.002 [DIRS 170150], MO0306MWDBGSMF.001 [DIRS 163816].

Note: Calculated in Excel file VA BDCF MC Correlations_Rev 4.x/s as shown in Appendix B. 
Table 6.2-8. Correlation Coefficients for the Volcanic Ash Scenario ICRP 72-Based BDCF Components

\begin{tabular}{|l|c|c|c|}
\hline Radionuclide & $\begin{array}{c}\text { External-Ingestion-Radon and } \\
\text { Short-term Inhalation }\end{array}$ & $\begin{array}{c}\text { Short-term Inhalation and } \\
\text { Long-term Inhalation }\end{array}$ & $\begin{array}{c}\text { External-Ingestion-Radon and } \\
\text { Long-term Inhalation }\end{array}$ \\
\hline${ }^{90} \mathrm{Sr}$ & 0 & 0.609 & 0 \\
\hline${ }^{99} \mathrm{Tc}$ & 0 & 0.609 & 0 \\
\hline${ }^{126} \mathrm{Sn}$ & 0.115 & 0.609 & 0.159 \\
\hline${ }^{137} \mathrm{Cs}$ & 0.102 & 0.609 & 0 \\
\hline${ }^{210} \mathrm{~Pb}$ & 0 & 0.609 & 0.097 \\
\hline${ }^{226} \mathrm{Ra}$ & 0 & 0.609 & 0 \\
\hline${ }^{227} \mathrm{Ac}$ & 0 & 0.609 & 0 \\
\hline${ }^{229} \mathrm{Th}$ & 0 & 0.609 & 0 \\
\hline${ }^{230} \mathrm{Th}$ & 0 & 0.609 & 0.102 \\
\hline${ }^{232} \mathrm{Th}$ & 0 & 0.609 & 0 \\
\hline${ }^{231} \mathrm{~Pa}$ & 0 & 0.609 & 0.081 \\
\hline${ }^{232} \mathrm{U}$ & 0 & 0.609 & 0 \\
\hline${ }^{233} \mathrm{U}$ & 0 & 0.609 & 0 \\
\hline${ }^{234} \mathrm{U}$ & 0 & 0.609 & 0 \\
\hline${ }^{236} \mathrm{U}$ & 0 & 0.609 & 0 \\
\hline${ }^{238} \mathrm{U}$ & 0 & 0.609 & 0 \\
\hline${ }^{237} \mathrm{~Np}$ & 0 & 0.609 & 0 \\
\hline${ }^{238} \mathrm{Pu}$ & 0 & 0.609 & 0 \\
\hline${ }^{239} \mathrm{Pu}$ & 0 & 0.609 & 0.609 \\
\hline${ }^{240} \mathrm{Pu}$ & 0 & 0.609 & 0 \\
\hline${ }^{242} \mathrm{Pu}$ & 0 & 0.609 & 0 \\
\hline${ }^{241} \mathrm{Am}$ & 0 & 0.609 & 0 \\
\hline${ }^{243} \mathrm{Am}$ & 0 & 0.699 & 0 \\
\hline${ }^{20} \mathrm{O}$ & 0 & 0 & 0 \\
\hline & 0 & 0 & 0 \\
\hline
\end{tabular}

Sources: MO0407SPAINEXI.002 [DIRS 170597], MO0407SPASRPBM.002 [DIRS 170755],

MO0403SPAAEIBM.002 [DIRS 169392], MO0407SPACRBSM.002 [DIRS 170677],

MO0406SPAETPBM.002 [DIRS 170150], MO0306MWDBGSMF.001 [DIRS 163816],

MO0503SPADCESR.000 [DIRS 172896].

Note: Calculated in Excel file VA BDCF MC Correlations_Rev 4.xIs as shown in Appendix B.

Some correlation coefficients in Tables 6.2-7 and 6.2-8 are shown as equal to zero. This absence of correlation between the variables was determined by performing a statistical test on the calculated value of the correlation coefficient. The hypothesis was that the (true) population correlation coefficient is equal to zero. If the calculated value of the correlation coefficient for the sampling distribution is equal to $r$, one can compute the values of a parameter $t$, such that

$$
t=\frac{r}{\sqrt{\frac{\left(1-r^{2}\right)}{n-2}}}
$$


where $n$ is the number of data points in the sampling distribution, and compare its value with Student's $t$ for $n$-2 degrees of freedom (Steel and Torrie 1980 [DIRS 150857], pp. 278 to 279). Table 6.2-9 lists the values of $t$ calculated from Equation 6.2-5 for different values of $r$. The hypothesis that the population correlation coefficient is equal to zero (no correlation) can be rejected at the $99 \%$ confidence level if the value of $t$ is less than 2.576 (Lide and Frederikse 1997 [DIRS 103178], p. A-105). (The one-tail area under the probability distribution function for variable $t$ is equal to 0.995 for $t=2.576$.) This corresponds to the value of $r$ equal to 0.0813 . Note that the distribution of $t$ approaches a normal distribution for the large number of degrees of freedom, which is the case here (998 degrees of freedom). The correlation coefficient was thus set to zero for the calculated values less than 0.0813 .

Table 6.2-9. Calculated Values of Correlation Coefficient and Variable $t$

\begin{tabular}{|c|c|}
\hline Calculated Correlation Coefficient, $r$ & $t$ \\
\hline 0.0000 & 0.000 \\
\hline 0.0200 & 0.632 \\
\hline 0.0400 & 1.265 \\
\hline 0.0600 & 1.899 \\
\hline 0.0610 & 1.931 \\
\hline 0.0620 & 1.962 \\
\hline 0.0630 & 1.994 \\
\hline 0.0700 & 2.217 \\
\hline 0.0780 & 2.472 \\
\hline 0.0790 & 2.504 \\
\hline 0.0800 & 2.535 \\
\hline 0.0810 & 2.567 \\
\hline 0.0812 & 2.574 \\
\hline 0.0813 & 2.577 \\
\hline 0.0820 & 2.599 \\
\hline 0.1000 & 3.175 \\
\hline 0.1200 & 3.819 \\
\hline 0.1400 & 4.467 \\
\hline 0.1600 & 5.121 \\
\hline 0.1800 & 5.781 \\
\hline 0.2000 & 6.449 \\
\hline
\end{tabular}

Source: Calculated in Excel file VA BDCF MC Correlations_Rev 4.x/s as shown in Appendix B.

The correlations between the BDCF components of different radionuclides have also been calculated. The correlation coefficients for the external-ingestion-radon component are listed in Tables 6.2-10 and 6.2-11 for the ICRP 30- and ICRP 72-based values, respectively. Correlation coefficients for short-term and long-term inhalation components are equal to 1 . Details of the calculations are presented in Appendix B. 


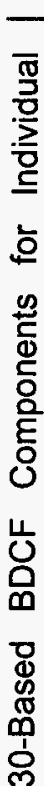

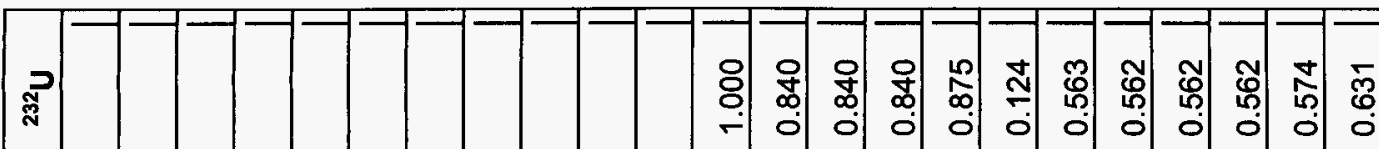

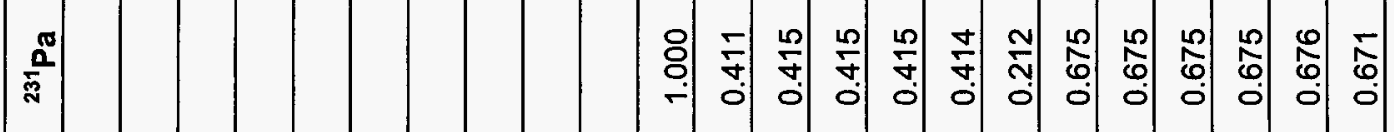

点

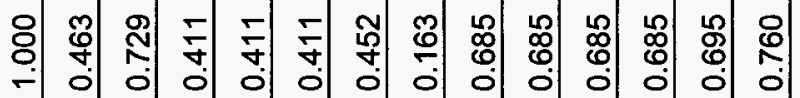

당

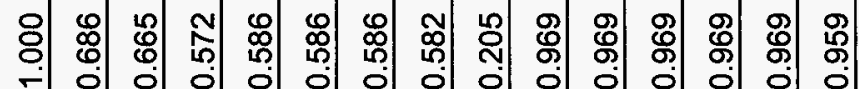

독

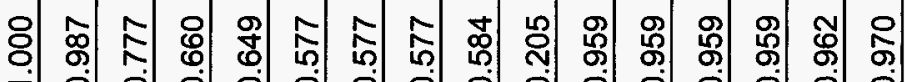

$\underline{\underline{\alpha}}$

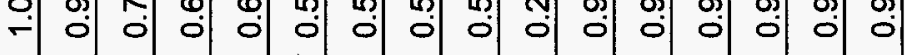

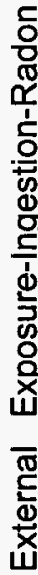

ลิ่

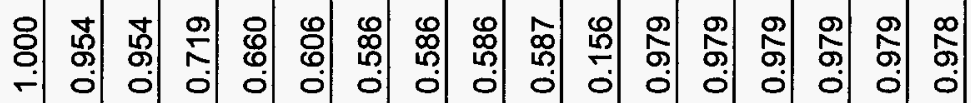

ส্

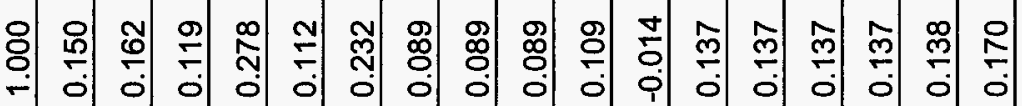

$\frac{0}{2}$

○్రం:

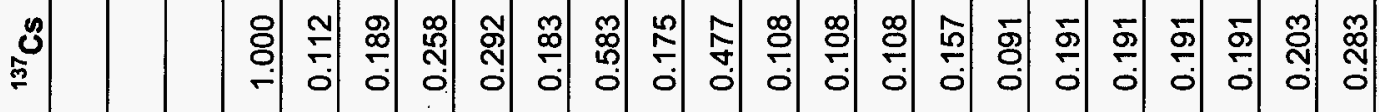

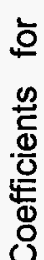

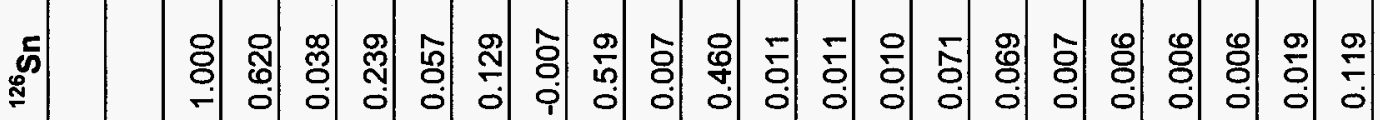

등 응

ㅎํㄹ

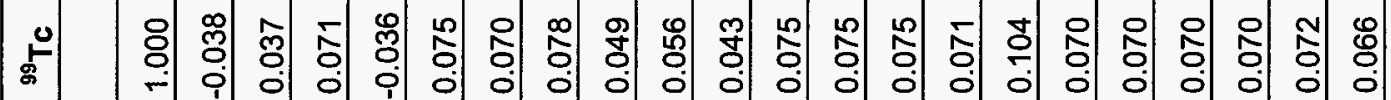

$+$

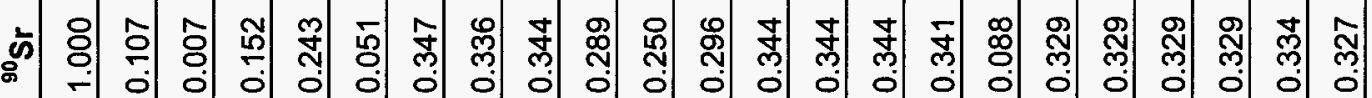

$\circ$
ஸे
0
$\frac{1}{0}$
$\frac{1}{\circ}$

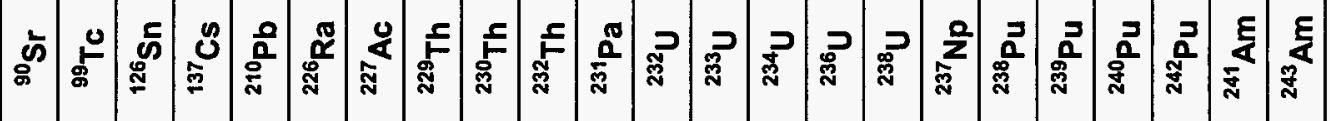




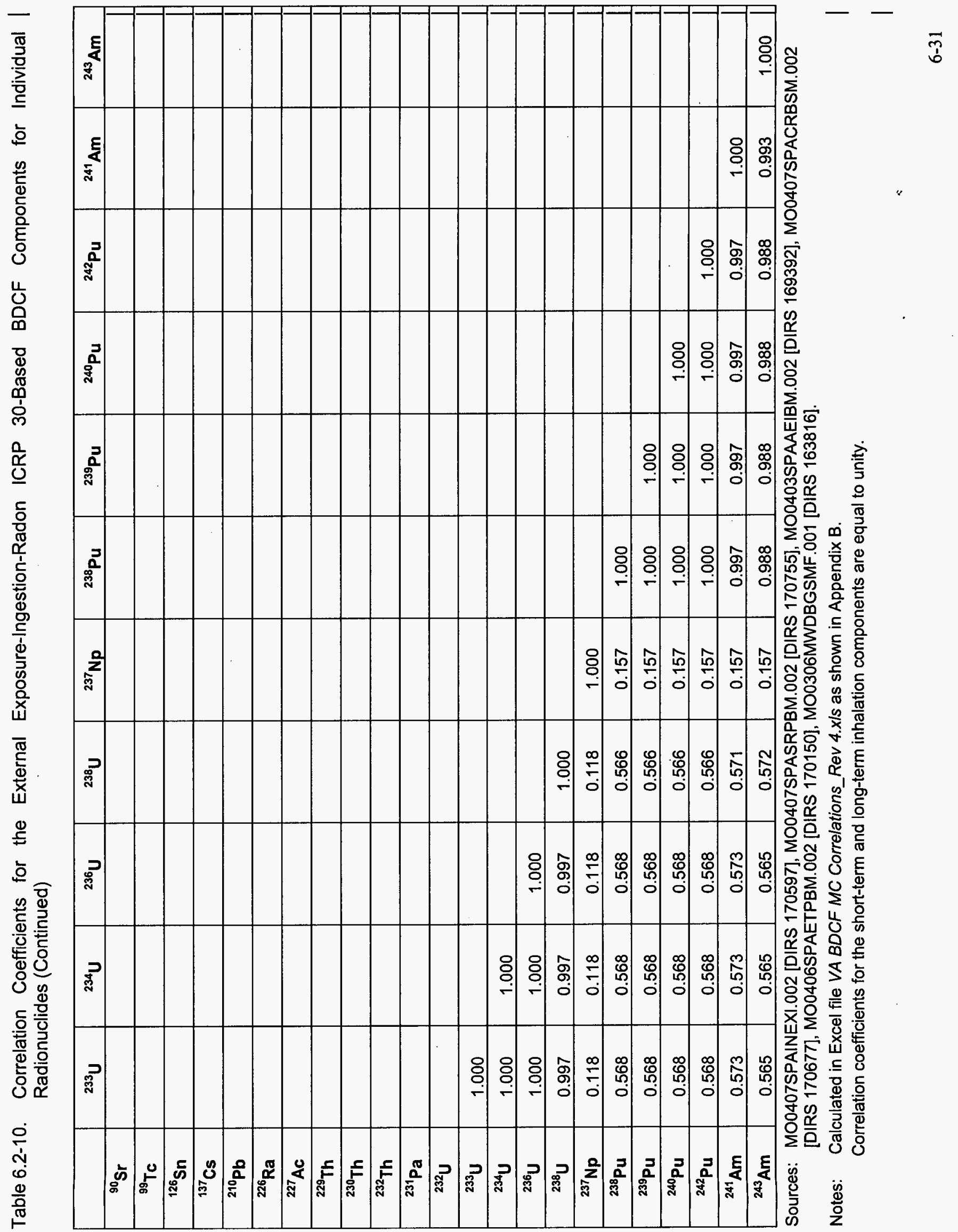




\begin{tabular}{|c|c|c|c|c|c|c|c|c|c|c|c|c|c|c|c|c|c|c|c|c|c|c|c|}
\hline$\stackrel{\text { \& }}{\underline{0}}$ & & & & & & & & & & & & 음 & $\begin{array}{l}9 \\
80 \\
0\end{array}$ & $\begin{array}{l}8 \\
8 \\
0 \\
0\end{array}$ & o: & 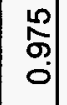 & $\begin{array}{l}\text { 怘 } \\
0 \\
0\end{array}$ & $\frac{0}{6}$ & $\frac{0}{50}$ & $\begin{array}{l}0 \\
i \\
0\end{array}$ & $\begin{array}{c}0 \\
i \frac{0}{0} \\
0\end{array}$ & 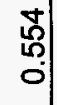 & $\underset{\mathbb{N}}{\mathbb{N}}$ \\
\hline$\frac{\AA}{\pi}$ & & & & & & & & & & & $\stackrel{8}{\circ}$ & $\begin{array}{c}\hat{\mathrm{o}} \\
\text { యे } \\
0\end{array}$ & $\begin{array}{c}10 \\
\dot{0} \\
0\end{array}$ & $\begin{array}{l}\frac{1}{2} \\
\dot{\sigma} \\
0\end{array}$ & 号 & $\mid \begin{array}{c}\tilde{D} \\
0 \\
0\end{array}$ & ন্ডু & $\begin{array}{l}\mathbf{T} \\
6 \\
0 \\
0\end{array}$ & $\begin{array}{l}\mathbf{t} \\
0 \\
0\end{array}$ & 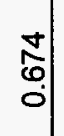 & $\begin{array}{l}\mathbb{T} \\
0 \\
0\end{array}$ & $\begin{array}{l}\mathbf{t} \\
\mathbf{b} \\
0\end{array}$ & $\begin{array}{l}\stackrel{2}{0} \\
\stackrel{0}{0} \\
0\end{array}$ \\
\hline 占 & & & & & & & & & & \& & $\stackrel{\substack{\infty \\
0}}{0}$ & 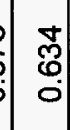 & 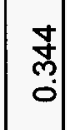 & \begin{tabular}{l}
\multirow{J}{*}{} \\
’.
\end{tabular} & 耪 & $\left|\begin{array}{c}10 \\
\tilde{n} \\
0 \\
0\end{array}\right|$ & $\begin{array}{l}\text { ᄋ } \\
\stackrel{0}{0}\end{array}$ & $\begin{array}{l}3 \\
0 \\
0 \\
0 \\
0\end{array}$ & $\begin{array}{l}0 \\
0 \\
0 \\
0\end{array}$ & $\begin{array}{l}0 \\
0 \\
0 \\
0 \\
0\end{array}$ & 总 & 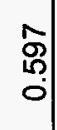 & $\begin{array}{l}\bar{N} \\
0\end{array}$ \\
\hline F్ & & & & & & & & & 음 & 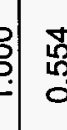 & 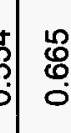 & 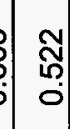 & $\mid \begin{array}{l}0 \\
0 \\
0 \\
0\end{array}$ & $\begin{array}{l}0 \\
\mathbb{0} \\
\stackrel{1}{0} \\
0\end{array}$ & $\mid \begin{array}{l}\mathscr{D} \\
0 \\
0 \\
0 \\
\mid\end{array}$ & 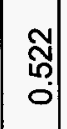 & స్ స్ & $\begin{array}{l}\text { : } \\
\text { o } \\
\text { : }\end{array}$ & $\begin{array}{l}\text { : } \\
\text { Oे } \\
0\end{array}$ & $\begin{array}{l}\text { : } \\
\stackrel{0}{0} \\
0\end{array}$ & $\begin{array}{l}\text { : } \\
\stackrel{\circ}{\circ} \\
\end{array}$ & 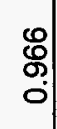 & $\begin{array}{c}N \\
\infty \\
0 \\
0\end{array}$ \\
\hline 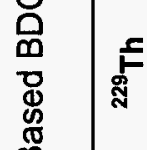 & & & & & & & & 8 & కి & 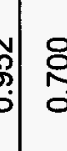 & $\begin{array}{lll} \\
0 \\
0\end{array}$ & \begin{tabular}{l}
\multirow{N}{*}{} \\
0 \\
0 \\
0
\end{tabular} & $\begin{array}{l}0 \\
0 \\
0 \\
0 \\
0\end{array}$ & 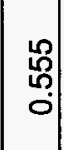 & $\mid \begin{array}{l}0 \\
0 \\
0 \\
0 \\
0\end{array}$ & $\left|\begin{array}{c}\infty \\
\overline{0} \\
0\end{array}\right|$ & $\stackrel{\infty}{\grave{o}}$ & 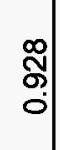 & 心్లి & 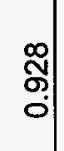 & 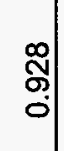 & $\begin{array}{l}\text { 寸 } \\
\stackrel{5}{\circ} \\
\circ\end{array}$ & $\begin{array}{l}\mathscr{8} \\
\tilde{\delta} \\
\delta\end{array}$ \\
\hline ลั & & & & & & & 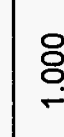 & : & 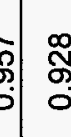 & 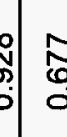 & $\begin{array}{l}0 \\
0 \\
0\end{array}$ & : & $\begin{array}{l}9 \\
8 \\
0 \\
0\end{array}$ & $\begin{array}{l}0 \\
0 \\
6 \\
0 \\
0\end{array}$ & $\mid \begin{array}{l}0 \\
0 \\
0 \\
0 \\
0\end{array}$ & $\mid \begin{array}{l}0 \\
8 \\
\varnothing \\
0\end{array}$ & $\frac{8}{0}$ & 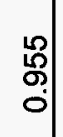 & $\begin{array}{l}\text { గొ } \\
\text { م } \\
0\end{array}$ & 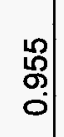 & $\begin{array}{l}0 \\
\stackrel{0}{\circ} \\
0\end{array}$ & 仓̊ & $\begin{array}{l}\text { Dू } \\
\text { ơ } \\
\text { d }\end{array}$ \\
\hline 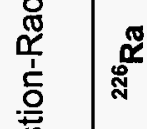 & & & & & & 8 & $\mid$\begin{tabular}{l}
\multirow{J}{*}{} \\
\multirow{2}{*}{}
\end{tabular} & 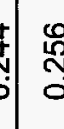 & 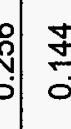 & 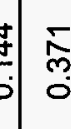 & $\stackrel{\substack{0 \\
0}}{\circ}$ & $\stackrel{\infty}{\infty}$ & $\frac{2}{0}$ & $\frac{+}{0}$ & 㽦 & 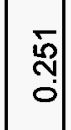 & ఫ্ & $\stackrel{\varrho}{\leftrightarrows}$ & $\frac{\Xi}{0}$ & $\frac{⿱ 亠 凶}{0}$ & $\frac{\Xi}{0}$ & $\stackrel{N}{\infty}$ & స్ ఊ్ \\
\hline$\frac{0}{0}$ & & & & & $\stackrel{8}{\circ}$ & 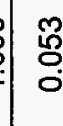 & ڤ్ & مु & $\bar{n}=\frac{1}{5}$ & $\stackrel{?}{?}$ & مِ & ড̦ & 임 & $\begin{array}{l}\text { : } \\
\stackrel{\text { s. }}{0}\end{array}$ & 을 & 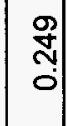 & $\frac{\stackrel{2}{\sigma}}{\circ}$ & ষ্ল & 。্ల్ & 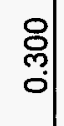 & 이 & $\begin{array}{l}\text { ఫ్ల } \\
0 \\
0\end{array}$ & ำ \\
\hline$\delta_{0}^{n}$ & & & & $\stackrel{8}{8}$ & $\frac{N}{5}$ & 怘 & $\mid \begin{array}{l}0 \\
0 \\
0\end{array}$ & 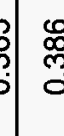 & $\stackrel{\substack{0 \\
:}}{\stackrel{\infty}{\circ}}$ & 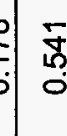 & 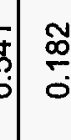 & $\begin{array}{l}5 \\
0 \\
0\end{array}$ & $\mid \begin{array}{l}8 \\
0 \\
0\end{array}$ & $\begin{array}{l}8 \\
\vdots \\
0\end{array}$ & $\begin{array}{l}8 \\
0 \\
0 \\
\end{array}$ & 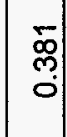 & $\stackrel{\text { के }}{\stackrel{0}{0}}$ & $\begin{array}{l}\infty \\
\stackrel{\infty}{\infty} \\
\vdots \\
0\end{array}$ & $\mid \begin{array}{l}\infty \\
\infty \\
\vdots \\
0\end{array}$ & 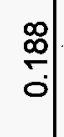 & $\mid \begin{array}{c}\infty \\
\infty \\
0 \\
0\end{array}$ & స్త్ & గొ \\
\hline జ్ & & & $\stackrel{8}{\circ}$ & 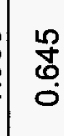 & $\stackrel{\substack{\infty \\
\hdashline \\
0}}{0}$ & 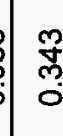 & So & 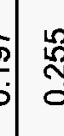 & \begin{tabular}{l|l}
$\stackrel{m}{0}$ \\
\hdashline \\
\hdashline
\end{tabular} & 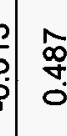 & 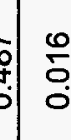 & 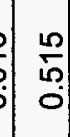 & $\begin{array}{l}8 \\
0 \\
0\end{array}$ & $\begin{array}{l}0 \\
8 \\
0 \\
0\end{array}$ & $\mid \begin{array}{l}0 \\
0 \\
0 \\
0\end{array}$ & $\begin{array}{l}\text { : } \\
\text { o. } \\
0\end{array}$ & $\stackrel{\stackrel{N}{N}}{\circ}$ & ণั) & ơ & ণิ & $\begin{array}{l}\widetilde{\delta} \\
0 \\
0\end{array}$ & $\begin{array}{l}\text { R } \\
0 \\
0 \\
0\end{array}$ & 志 \\
\hline$\stackrel{\cup}{\circ}$ & & $\underset{8}{8}$ & $\begin{array}{l}\text { o } \\
\\
0\end{array}$ & $\underset{\delta}{\mathscr{\delta}}$ & $\underset{0}{\bar{\Lambda}}$ & 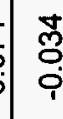 & $\stackrel{8}{8}$ & : & 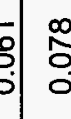 & $\begin{array}{l}0 \\
0 \\
0\end{array}$ & 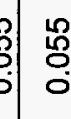 & : & $\mid \begin{array}{c}n \\
0 \\
0 \\
0\end{array}$ & $\begin{array}{l}n \\
0 \\
0 \\
0\end{array}$ & \begin{tabular}{|l|}
0 \\
0 \\
0 \\
0
\end{tabular} & $\begin{array}{l}9 \\
0 \\
0\end{array}$ & $\frac{8}{\circ}$ & 영 & $\begin{array}{l}0 \\
\\
0 \\
0\end{array}$ & 옹 & $\begin{array}{l}0 \\
0 \\
0 \\
0\end{array}$ & $\begin{array}{l}0 \\
\vdots \\
0 \\
0\end{array}$ & $\begin{array}{l}0 \\
\text { ర్ } \\
0\end{array}$ \\
\hline চু & 임 & 午 & $\begin{array}{l}0 \\
\stackrel{0}{0} \\
0 \\
0\end{array}$ & $\frac{\vec{\sigma}}{\stackrel{5}{\circ}}$ & 㥑 & 8 & $\mid \begin{array}{l}0 \\
0 \\
0\end{array}$ & $\begin{array}{l}\hat{m} \\
0 \\
0\end{array}$ & \begin{tabular}{lll}
$\tilde{b}$ \\
$\vdots$ \\
\hdashline
\end{tabular} & 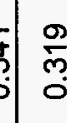 & 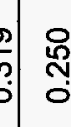 & : & $\begin{array}{c}\bar{\sigma} \\
\tilde{0} \\
0\end{array}$ & $\begin{array}{c}\text { जै } \\
\text { ర్ } \\
0\end{array}$ & $\left|\begin{array}{c}\text { N } \\
\text { W. } \\
0\end{array}\right|$ & స్త & $\begin{array}{l}\text { : } \\
\text { O } \\
0\end{array}$ & $\begin{array}{l}\text { స్లి } \\
\text { ه }\end{array}$ & 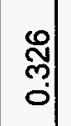 & $\begin{array}{c}\widehat{్} \\
0 \\
0\end{array}$ & స్ల & 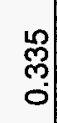 & 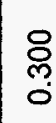 \\
\hline s & চ্ঠ) & 岕 & 离 & 造 & $\frac{0}{0}$ & 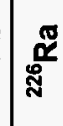 & $\frac{\mathscr{Q}}{\alpha}$ & 占 & 实 & 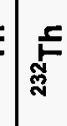 & $=\frac{\mathbb{R}^{\infty}}{\mathrm{N}}$ & 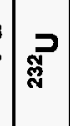 & ग. & 两 & 疋 & 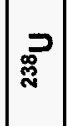 & 高 & 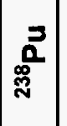 & 胥 & 豆 & 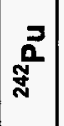 & $\frac{\xi}{\frac{E}{\alpha}}$ & $\frac{\xi}{8}$ \\
\hline
\end{tabular}




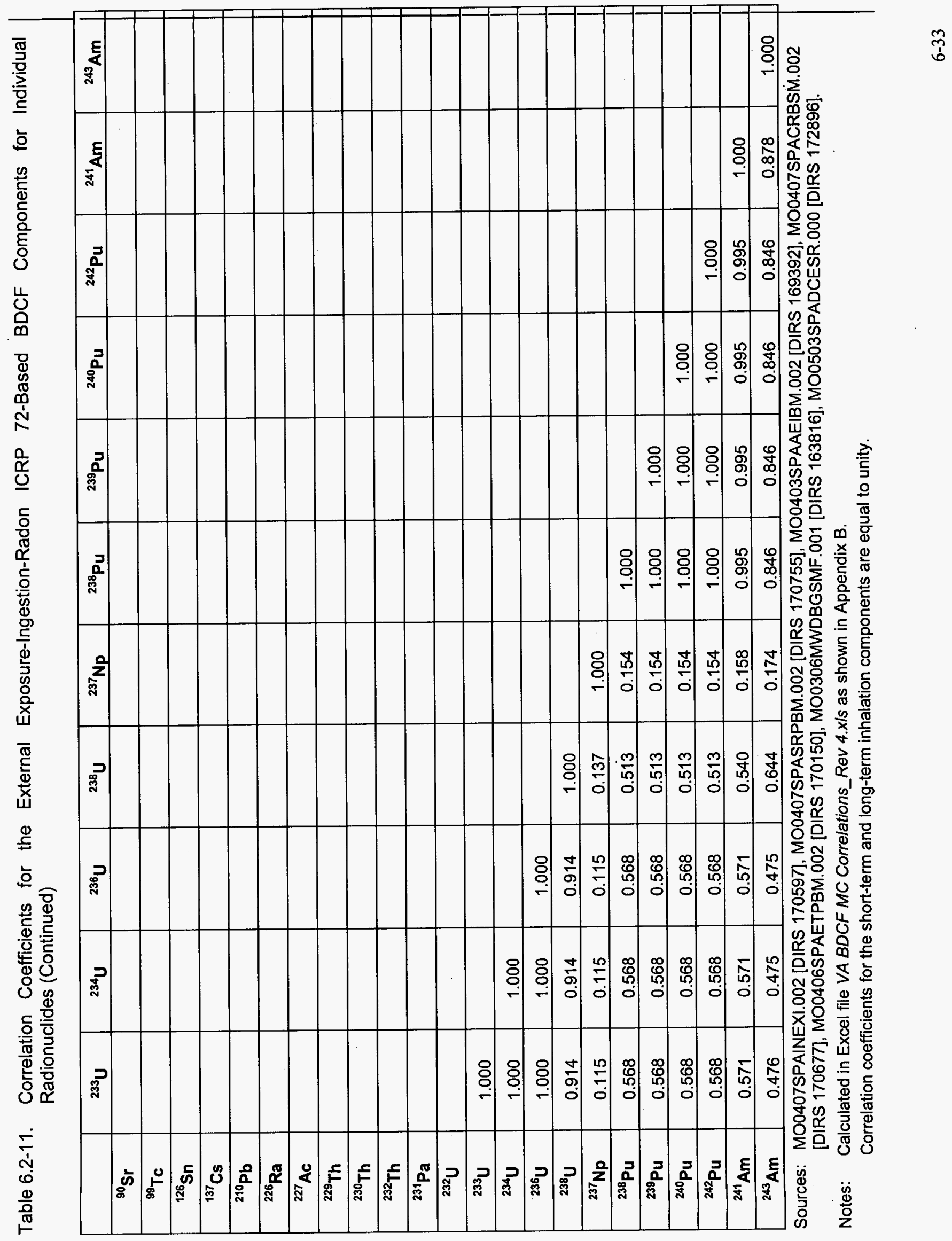


Generally, the correlation coefficients for the external exposure-radon-ingestion BDCF components are the highest for actinides $\left({ }^{227} \mathrm{Ac}\right.$ and heavier radionuclides) and lower for lighter radionuclides. The correlations in this case depend on the importance of individual pathways, and input parameters for these pathways.

\subsubsection{Pathway Analysis}

The exposure pathway contributions to the BDCFs for volcanic ash exposure scenario are time-dependent and also depend on the thickness of contaminated ash layer. The pathway analysis was first conducted for the initial ash deposition at $t=0$ before the mass loading decrease occurs, and for the ash layer thickness equal to or less than the critical thickness $\left(d_{a} \leq d_{c}\right)$. For such conditions, the values functions $f(t)$ and $g\left(d_{a}\right)$ in Equations 6.2-1 to 6.2-3 are equal to unity. From Equations $6.2-1$ to $6.2-3$, when $t=0$ and $d_{a} \leq d_{c}$, then

$$
B D C F_{i}\left(d_{a}, t\right)=B D C F_{e x t, i n g, R n, i}+B D C F_{i n h, l, i}+B D C F_{i n h, p, i}
$$

The percentage contributions of individual exposure pathways to the mean volcanic ash exposure scenario BDCFs at $t=0$ and $d_{a} \leq d_{c}$ are listed in Tables 6.2-12 and 6.2-13 for the ICRP 30- and ICRP 72-based BDCF values, respectively.

The evolution of BDCFs with time, which is controlled by the function $f(t)$ shown in Equation 6.2-2, affects only the short-term inhalation component (see BSC 2004 [DIRS 169458], Section 6.3, for the development and detailed description of this function). The short-term inhalation component accounts for about one-third of the BDCF values presented in Tables 6.2-12 and 6.2-13 for all actinides. For those radionuclides, the total mean BDCFs show a greater degree of reduction over time compared to radionuclides with smaller short-term inhalation components. This effect is shown in Figure 6.2-3 for ash thickness equal to $1 \mathrm{~cm}$ $(10 \mathrm{~mm})$ and the average value of the critical thickness $\left(d_{c}=2 \mathrm{~mm}\right.$ ) (see Excel file $V A B D C F$ MC Pathway Analysis_Rev 4.xls in Appendix B). For such conditions, Equation 6.2-1 becomes

$$
B D C F_{i}(t)=B D C F_{e x t, i n g, R n, i}+\left(B D C F_{i n h, v, i} e^{-0.125 y r^{-1} t}+B D C F_{i n h, p, i}\right) \frac{2 m m}{10 m m}
$$

where $0.125 \mathrm{yr}^{-1}$ is the value of mass loading decrease constant for ash thickness $\geq 10 \mathrm{~mm}$ (BSC 2004 [DIRS 169458], Section 7.1). The graph was plotted for ICRP 72-based BDCFs for ${ }^{90} \mathrm{Sr},{ }^{226} \mathrm{Ra}$, and ${ }^{239} \mathrm{Pu}$ because of the differing pathway contributions for these radionuclides. The analogous graph for the ICRP 30-based BDCFs shows a similar behavior of BDCFs with time (see Excel file VA BDCF MC Pathway Analysis_Rev3.xls in Appendix B). The mean BDCF for ${ }^{90} \mathrm{Sr}$ includes significant contributions from the external, inhalation, and ingestion exposure pathways. Ingestion is a relatively insignificant pathway for ${ }^{226} \mathrm{Ra}$, while the mean BDCF for ${ }^{239} \mathrm{Pu}$ is dominated by inhalation. 
휸 스

-

흥

$+-1$

₹

苟

氙

苞 N N

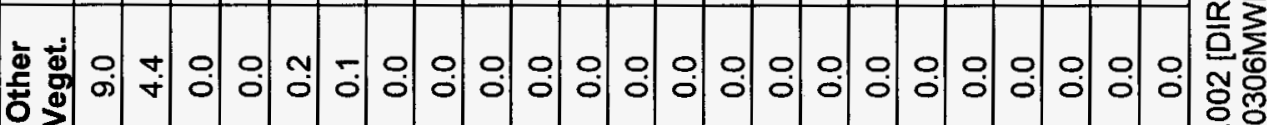

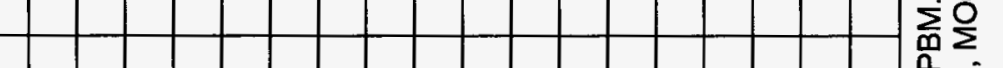
歪焉 自

흔

क象点

$\frac{5}{2}$

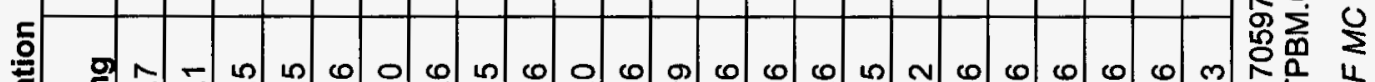

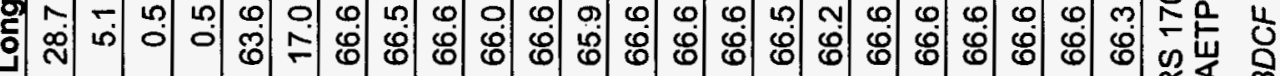
$\underline{\underline{\underline{E}}}$

ก +

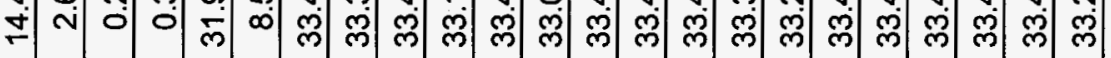

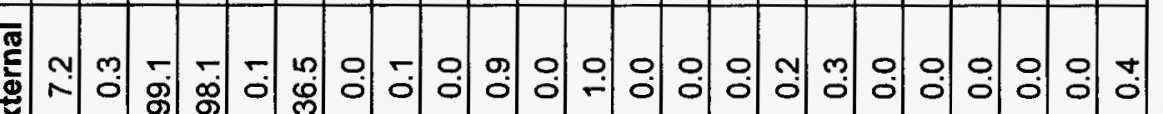
产 


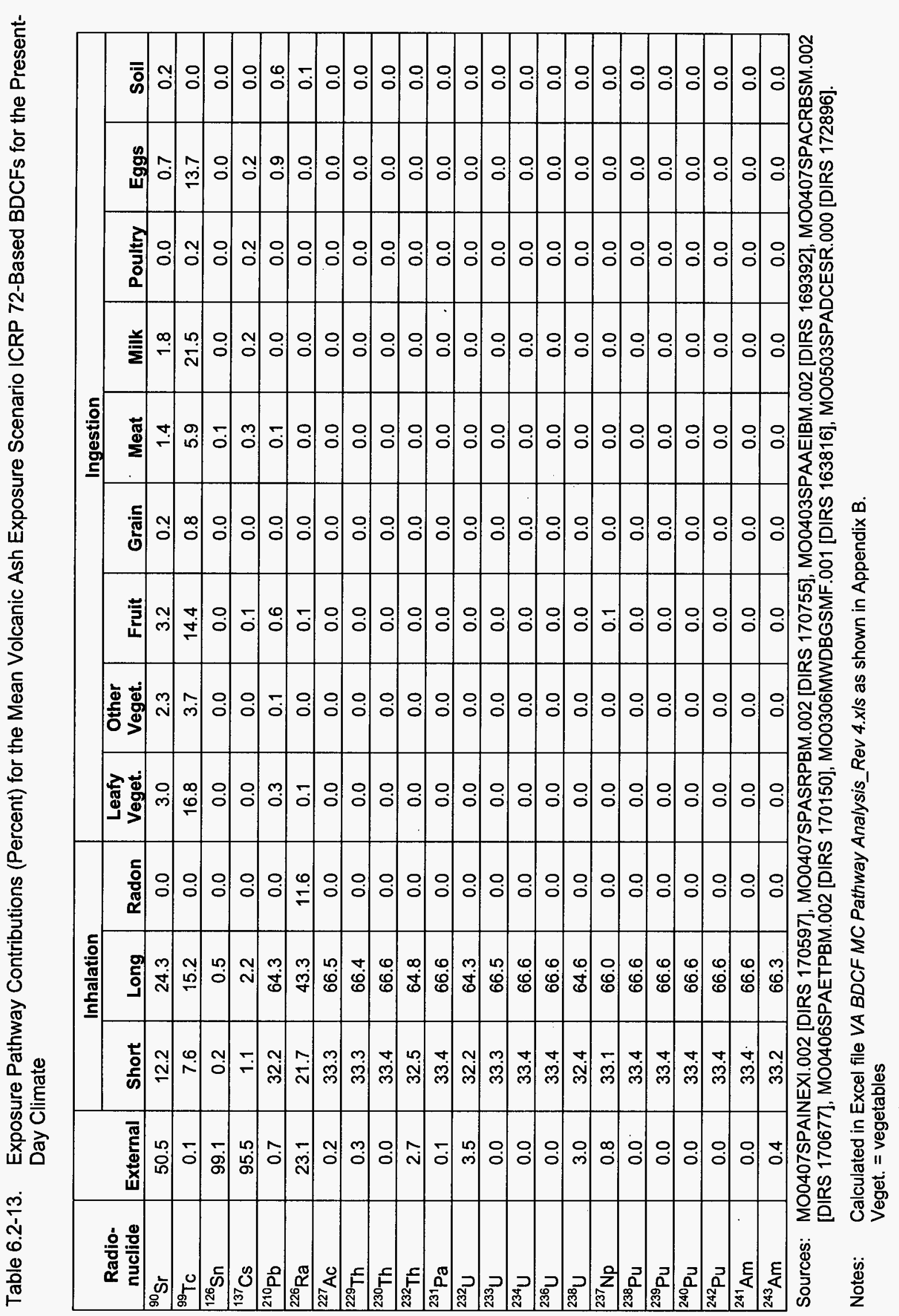




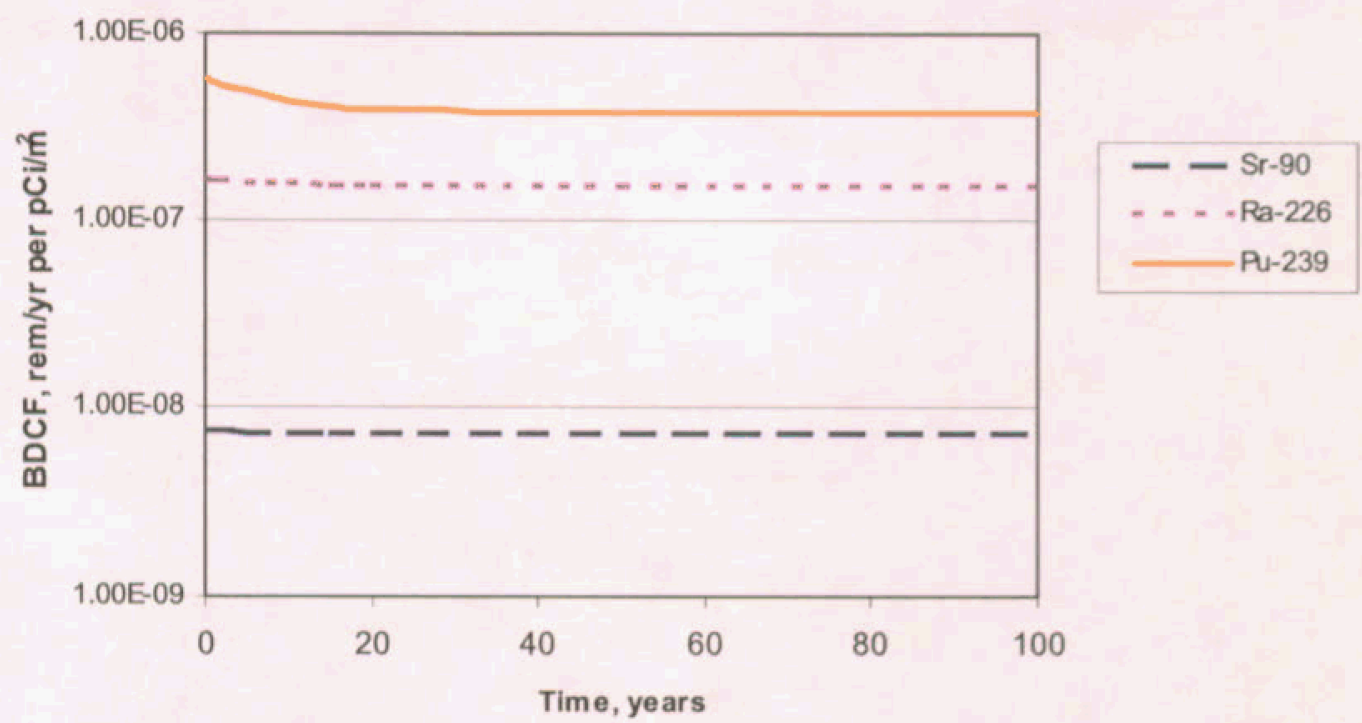

Sources: MO0407SPAINEXI.002 [DIRS 170597], MO0407SPASRPBM.002 [DIRS 170755] MO0403SPAAEIBM.002 [DIRS 169392], MO0407SPACRBSM.002 [DIRS 170677], MO0406SPAETPBM.002 [DIRS 170150], MO0306MWDBGSMF.001 [DIRS 163816].

Note: $\quad$ Calculated in Excel file VA BDCF MC Pathway Analysis_Rev 4.x/s (Appendix B); ash thickness $=10 \mathrm{~mm}$, critical thickness $=2 \mathrm{~mm}$.

Figure 6.2-3. Mean BDCFs as a Function of Time

The short-term and the long-term inhalation components of BDCFs are affected by the thickness of the layer of contaminated ash. When the contaminated ash layer thickness is greater than the critical thickness, a fraction of the deposited activity is unavailable for resuspension; only the fraction equal to $d_{d} / d_{a}$ can become resuspended. That is why the inhalation BDCF components are multiplied by $d_{c} / d_{a}$ when $d_{a}>d_{c}$. For such conditions, when the ash thickness increases, a smaller fraction of the deposited activity would be available for resuspension because an increasing fraction would be in ash that is not subject to resuspension (i.e., below the critical thickness boundary). Consequently, the inhalation contribution would decrease. This effect, which is illustrated in Figure 6.2-4, would be strongest for radionuclides with large BDCF contribution from inhalation, such as ${ }^{239} \mathrm{Pu}$. Figure 6.2-4 shows the BDCFs for the selected radionuclides as a function of ash thickness for the point-in-time conditions at $t=0$ and for the maximum value of the critical thickness $\left(d_{c}=3 \mathrm{~mm}\right.$ ) (see Excel file $V A$ BDCF MC Pathway Analysis_Rev 4.xls in Appendix B). For such conditions, Equation 6.2-1 becomes

$$
B D C F_{i}\left(d_{a}\right)=B D C F_{e x t, i n g, R n, i}+\left(B D C F_{i n h, v, i}+B D C F_{i n h, p, i}\right) g\left(d_{a}\right)
$$

and the function of ash thickness $g\left(d_{a}\right)$, is expressed as

$$
g\left(d_{a}\right)=\left\{\begin{array}{cl}
1 & \text { when } d_{a}<3 \mathrm{~mm} \\
\frac{3 \mathrm{~mm}}{d_{a}} & \text { when } d_{a} \geq 3 \mathrm{~mm}
\end{array}\right.
$$




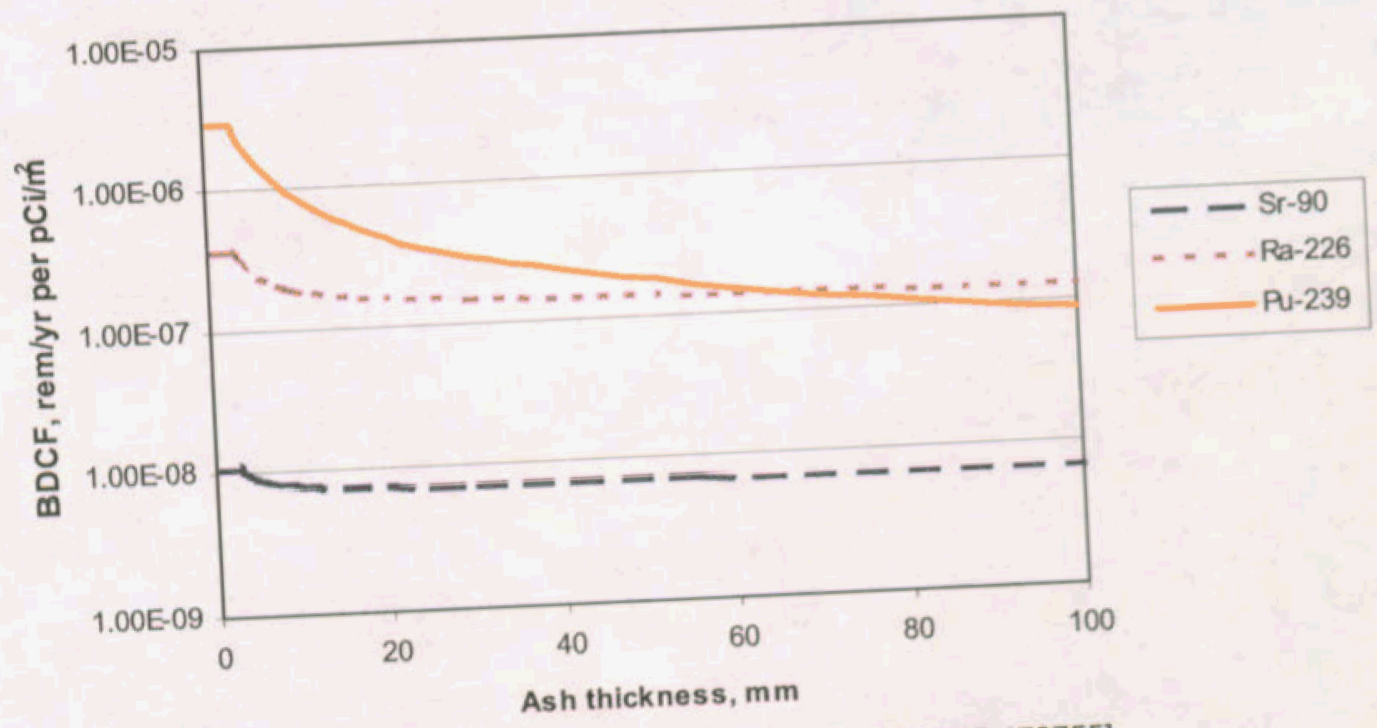

Sources: MO0407SPAINEXI.002 [DIRS 170597], MO0407SPASRPBM.002 [DIRS 170755], MO0403SPAAEIBM.002 [DIRS 169392], MO0407SPACRBSM.002 [DIRS 17 001 [DIRS 163816] MO0406SPAETPBM.002 [DIRS 170150], MO0306MWDBGSMF.001 [DIRS 163816 .

Calculated in Excel file VA BDCF MC Pathway Analysis_Rev 4.xIs (Appendix B); critical thickness = $3 \mathrm{~mm}$,

Note: time $=0 \mathrm{yr}$.

Figure 6.2-4. Mean BDCFs as a Function of Ash Thickness

The maximum value of the critical thickness was used for the plots in Figure 6.2-4 to better show the "shoulder" of the curves, i.e., the region for $d_{a}<d_{c}$ where the BDCF does not depend on the ash thickness because the function $g\left(d_{a}\right)$ is constant.

The analogous graph for the ICRP 30-based BDCFs shows a similar behavior of BDCFs with ash thickness (see Excel file VA BDCF MC Pathway Analysis_Rev 3.xls in Appendix B).

\subsubsection{Climate Change}

Annual doses arising from volcanic eruption are calculated by combining the biosphere component (BDCFs) with the source term, i.e., the activity concentration of a given radionuclide per unit area (Equation 6.2-4). BDCFs are independent of the source term. The discussion of climate change presented here concerns only the biosphere component, the BDCFs, because the source term is calculated in the TSPA model.

A single biosphere model input parameter is affected by climate change: the growing time of crops for the human and animal consumption, which potentially may affect the ingestion exposure pathway. The ingestion exposure pathway contributes little to the BDCF for most radionuclides (Tables 6.2-12 and 6.2-13). The importance of the ingestion pathway gradually increases as the short-term inhalation BDCF component decreases.

Because climate change affects only the BDCF component associated with ingestion through the growing-time parameter, the value of this component was calculated for a future climate represented by the upper bound of the glacial transition climate. The results, in term of the ratios of the mean pathway BDCF values for the present-day and future climates, are presented in 
Table 6.2-14 and 6.2-15 for the ICRP 30- and ICRP 72-based BDCF values, respectively. These results indicate that the BDCFs for the volcanic release of radionuclides are relatively insensitive to the climate change.

The values in the table are ratios of the pathway BDCFs for the future and present-day climates. Since there is no difference between the inhalation, external exposure, and radon pathway contributions to BDCF for the present-day and future climates (climate-dependent parameters are not used to develop their values) and the difference between the ingestion pathway contributions are insignificant, it is recommended that the BDCFs developed for the present-day climate be used for the future climates.

Table 6.2-14. Ratio of Mean Ingestion Pathway ICRP 30-Based BDCFs for the Volcanic Ash Exposure I Scenario and the Future and Present-Day Climates

\begin{tabular}{|l|c|c|c|c|c|c|c|c|}
\hline \multirow{2}{*}{ Radionuclide } & \multicolumn{6}{|c|}{ Ingestion Pathway BDCF Ratio (Future Climate to Present-Day Climate) } \\
\cline { 2 - 9 } & $\begin{array}{c}\text { Leafy } \\
\text { Veget. }\end{array}$ & $\begin{array}{c}\text { Other } \\
\text { Veget. }\end{array}$ & Fruit & Grain & Meat & Milk & Poultry & Eggs \\
\hline${ }^{90} \mathrm{Sr}$ & 1.00 & 1.00 & 1.00 & 1.00 & 1.00 & 1.00 & 1.00 & 1.00 \\
\hline${ }^{99} \mathrm{Tc}$ & 1.00 & 1.00 & 1.00 & 1.00 & 1.00 & 1.00 & 1.00 & 1.00 \\
\hline${ }^{126} \mathrm{Sn}$ & 1.00 & 1.00 & 1.00 & 1.00 & 1.00 & 1.00 & 1.00 & 1.00 \\
\hline${ }^{137} \mathrm{Cs}$ & 1.00 & 1.00 & 1.00 & 1.00 & 1.00 & 1.00 & 1.00 & 1.00 \\
\hline${ }^{210} \mathrm{~Pb}$ & 1.00 & 0.99 & 1.01 & 1.00 & 1.00 & 1.00 & 1.00 & 1.00 \\
\hline${ }^{226} \mathrm{Ra}$ & 1.00 & 1.00 & 1.01 & 1.00 & 1.00 & 1.00 & 1.00 & 1.00 \\
\hline${ }^{227} \mathrm{Ac}$ & 1.00 & 0.98 & 1.02 & 1.00 & 1.00 & 1.00 & 1.00 & 1.00 \\
\hline${ }^{229} \mathrm{Th}$ & 1.00 & 0.98 & 1.02 & 1.00 & 0.99 & 0.99 & 1.00 & 1.00 \\
\hline${ }^{230} \mathrm{Th}$ & 1.00 & 1.02 & 0.98 & 1.00 & 1.01 & 1.01 & 1.00 & 1.00 \\
\hline${ }^{232} \mathrm{Th}+{ }^{228} \mathrm{Ra}+{ }^{228} \mathrm{Th}$ & 1.00 & 0.99 & 1.01 & 1.00 & 1.00 & 1.00 & 1.00 & 1.00 \\
\hline${ }^{231} \mathrm{~Pa}$ & 1.00 & 0.99 & 1.01 & 1.00 & 1.00 & 1.00 & 1.00 & 1.00 \\
\hline${ }^{232} \mathrm{U}+{ }^{228} \mathrm{Th}$ & 1.00 & 0.99 & 1.01 & 1.00 & 1.00 & 1.00 & 1.00 & 1.00 \\
\hline${ }^{233} \mathrm{U}$ & 1.00 & 0.99 & 1.01 & 1.00 & 1.00 & 1.00 & 1.00 & 1.00 \\
\hline${ }^{234} \mathrm{U}$ & 1.00 & 0.99 & 1.01 & 1.00 & 1.00 & 1.00 & 1.00 & 1.00 \\
\hline${ }^{236} \mathrm{U}$ & 1.00 & 0.99 & 1.01 & 1.00 & 1.00 & 1.00 & 1.00 & 1.00 \\
\hline${ }^{238} \mathrm{U}$ & 1.00 & 0.99 & 1.01 & 1.00 & 1.00 & 1.00 & 1.00 & 1.00 \\
\hline${ }^{237} \mathrm{~Np}$ & 1.00 & 1.00 & 1.00 & 1.00 & 1.00 & 1.00 & 1.00 & 1.00 \\
\hline${ }^{238} \mathrm{Pu}$ & 1.00 & 0.97 & 1.02 & 1.00 & 0.99 & 0.99 & 1.00 & 1.00 \\
\hline${ }^{239} \mathrm{Pu}$ & 1.00 & 0.97 & 1.02 & 1.00 & 0.99 & 0.99 & 1.00 & 1.00 \\
\hline${ }^{240} \mathrm{Pu}$ & 1.00 & 0.97 & 1.02 & 1.00 & 0.99 & 0.99 & 1.00 & 1.00 \\
\hline${ }^{242} \mathrm{Pu}$ & 1.00 & 0.97 & 1.02 & 1.00 & 0.99 & 0.99 & 1.00 & 1.00 \\
\hline${ }^{241} \mathrm{Am}$ & 1.00 & 0.97 & 1.02 & 1.00 & 0.99 & 0.99 & 1.00 & 1.00 \\
\hline${ }^{243} \mathrm{Am}$ & 1.00 & 0.97 & 1.02 & 1.00 & 0.99 & 0.99 & 1.00 & 1.00 \\
\hline
\end{tabular}

Sources: MO0407SPAINEXI.002 [DIRS 170597], MO0407SPASRPBM.002 [DIRS 170755], MO0403SPAAEIBM.002 [DIRS 169392], MO0407SPACRBSM.002 [DIRS 170677], MO0406SPAETPBM.002 [DIRS 170150], MO0306MWDBGSMF.001 [DIRS 163816].

Notes: Calculated in Excel file VA BDCF FC Pathway Analysis_Rev 3.xls as shown in Appendix B. Veget. $=$ vegetables 
Table 6.2-15. Ratio of Mean Ingestion Pathway ICRP 72-Based BDCFs for the Volcanic Ash Exposure Scenario and the Future and Present-Day Climates

\begin{tabular}{|l|c|c|c|c|c|c|c|c|}
\hline \multirow{2}{*}{ Radionuclide } & \multicolumn{6}{|c|}{ Ingestion Pathway BDCF Ratio (Future Climate to Present-Day Climate) } \\
\cline { 2 - 9 } & $\begin{array}{c}\text { Leafy } \\
\text { Veget. }\end{array}$ & $\begin{array}{c}\text { Other } \\
\text { Veget. }\end{array}$ & Fruit & Grain & Meat & Milk & Poultry & Eggs \\
\hline${ }^{90} \mathrm{Sr}$ & 1.00 & 1.00 & 1.00 & 1.00 & 1.00 & 1.00 & 1.00 & 1.00 \\
\hline${ }^{99} \mathrm{TC}$ & 1.00 & 1.00 & 1.00 & 1.00 & 1.00 & 1.00 & 1.00 & 1.00 \\
\hline${ }^{126} \mathrm{Sn}$ & 1.00 & 1.00 & 1.00 & 1.00 & 1.00 & 1.00 & 1.00 & 1.00 \\
\hline${ }^{137} \mathrm{Cs}$ & 1.00 & 1.00 & 1.00 & 1.00 & 1.00 & 1.00 & 1.00 & 1.00 \\
\hline${ }^{210} \mathrm{~Pb}$ & 1.00 & 0.99 & 1.01 & 1.00 & 1.00 & 1.00 & 1.00 & 1.00 \\
\hline${ }^{226} \mathrm{Ra}$ & 1.00 & 1.00 & 1.01 & 1.00 & 1.00 & 1.00 & 1.00 & 1.00 \\
\hline${ }^{227} \mathrm{Ac}$ & 1.00 & 0.98 & 1.02 & 1.00 & 1.00 & 1.00 & 1.00 & 1.00 \\
\hline${ }^{229} \mathrm{Th}$ & 1.00 & 0.98 & 1.02 & 1.00 & 0.99 & 0.99 & 1.00 & 1.00 \\
\hline${ }^{230} \mathrm{Th}$ & 1.00 & 1.02 & 0.98 & 1.00 & 1.01 & 1.01 & 1.00 & 1.00 \\
\hline${ }^{232} \mathrm{Th}{ }^{228} \mathrm{Ra}^{231} \mathrm{~Pa}$ & 1.00 & 1.00 & 1.01 & 1.00 & 1.00 & 1.00 & 1.00 & 1.00 \\
\hline${ }^{232} \mathrm{U}+{ }^{228} \mathrm{Th}$ & 1.00 & 0.99 & 1.01 & 1.00 & 1.00 & 1.00 & 1.00 & 1.00 \\
\hline${ }^{233} \mathrm{U}$ & 1.00 & 0.99 & 1.01 & 1.00 & 1.00 & 1.00 & 1.00 & 1.00 \\
\hline${ }^{234} \mathrm{U}$ & 1.00 & 0.99 & 1.01 & 1.00 & 1.00 & 1.00 & 1.00 & 1.00 \\
\hline${ }^{236} \mathrm{U}$ & 1.00 & 0.99 & 1.01 & 1.00 & 1.00 & 1.00 & 1.00 & 1.00 \\
\hline${ }^{238} \mathrm{U}$ & 1.00 & 0.99 & 1.01 & 1.00 & 1.00 & 1.00 & 1.00 & 1.00 \\
\hline${ }^{237} \mathrm{~Np}$ & 1.00 & 0.99 & 1.01 & 1.00 & 1.00 & 1.00 & 1.00 & 1.00 \\
\hline${ }^{238} \mathrm{Pu}$ & 1.00 & 1.00 & 1.00 & 1.00 & 1.00 & 1.00 & 1.00 & 1.00 \\
\hline${ }^{239} \mathrm{Pu}$ & 1.00 & 0.97 & 1.02 & 1.00 & 0.99 & 0.99 & 1.00 & 1.00 \\
\hline${ }^{240} \mathrm{Pu}$ & 1.00 & 0.97 & 1.02 & 1.00 & 0.99 & 0.99 & 1.00 & 1.00 \\
\hline${ }^{242} \mathrm{Pu}$ & 1.00 & 0.97 & 1.02 & 1.00 & 0.99 & 0.99 & 1.00 & 1.00 \\
\hline${ }^{241} \mathrm{Am}$ & 1.00 & 0.97 & 1.02 & 1.00 & 0.99 & 0.99 & 1.00 & 1.00 \\
\hline${ }^{243} \mathrm{Am}$ & 1.00 & 0.97 & 1.02 & 1.00 & 0.99 & 0.99 & 1.00 & 1.00 \\
\hline${ }^{20 u}$ & 1.00 & 0.97 & 1.02 & 1.00 & 0.99 & 0.99 & 1.00 & 1.00 \\
\hline
\end{tabular}

Sources: MO0407SPAINEXI.002 [DIRS 170597], MO0407SPASRPBM.002 [DIRS 170755], MO0403SPAAEIBM.002 [DIRS 169392], MO0407SPACRBSM.002 [DIRS 170677], MO0406SPAETPBM.002 [DIRS 170150], MO0306MWDBGSMF.001 [DIRS 163816], MO0503SPADCESR.000 [DIRS 172896].

Notes: $\quad$ Calculated in Excel file VA BDCF FC Pathway Analysis_Rev 4.x/s as shown in Appendix B.

Veget. $=$ vegetables

\subsection{INHALATION DOSE FACTORS FOR THE ERUPTION PHASE}

The eruption phase of a volcanic event refers to the conditions that exist during the volcanic eruption, before the deposition of volcanic ash on the ground is completed. This phase is not included in the calculation of BDCFs for volcanic ash exposure scenario because the BDCFs are calculated for the conditions that exist after the deposition took place. BDCFs include inhalation exposure to resuspended ash and contaminated soil, external exposure, and ingestion exposure and are calculated on the annual basis, regardless of the actual eruption time and day. The eruption phase is treated separately and its consequences are evaluated as those arising from the exposure occurring during an event of a limited duration (acute or near-acute exposure), rather than from a long-term, chronic exposure thereafter. The latter is evaluated using the BDCFs. 
The inhalation dose factor may be used in TSPA assessments to evaluate whether the doses received by the RMEI during an eruption need to be included in calculation of the expected dose.

Because during the eruption higher concentrations of airborne radioactive particulates are expected, inhalation of airborne contaminated ash particles is the only pathway considered in the analysis for this phase. The other possible pathways, such as external exposure from contaminated ash, are inherently included in the BDCFs (Section 6.2). Inhalation exposure arising from direct gaseous volcanic emissions was not considered because gaseous radionuclides were not included among radionuclides of interest (Section 6.1.1).

This section describes the development of the inhalation dose factor for the eruption phase (Section 6.3.2). To put the eruption phase into perspective, Section 6.3.1 contains a summary of mass loading measurements taken during volcanic eruptions. These values are presented for reference only but one could compare them with the values of mass loading used as input for the biosphere model to calculate volcanic ash scenario BDCFs (Table 4.1-1). It needs to be noted, however, that the mass loading values used in the biosphere model represent annual average conditions, while the values summarized in Section 6.3.1 usually represent instantaneous or short-term conditions.

\subsubsection{Mass Loading Levels During Volcanic Eruption}

This section is a summary of airborne particle concentration measurements taken during and immediately after volcanic eruptions. This summary is provided to develop an understanding of airborne concentrations of ash that may occur at the location of the receptor following a volcanic eruption at Yucca Mountain. This information may be used to support evaluation of the dose to the RMEI during a volcanic eruption, since the biosphere model does not evaluate the dose during the period of active ash fall. Inhalation dose from airborne particulate concentrations during volcanic eruption, if necessary, will be calculated as a component of performance assessment. The data presented in this section were not further used in this analysis.

The data in this section are from four volcanic eruptions at four widely spaced locations. Data at each location include measurements made over time and in different local collecting sites. Most of the measurements reported in this section were taken at ambient monitoring stations during or soon after ash-fall events. Ambient monitoring stations usually are centrally located in communities. The concentrations of airborne particles measured at those stations are representative of regional or local conditions that are not influenced by specific, immediately adjacent activities.

Mount St. Helens-Total suspended particulate (TSP) concentrations at Yakima, Washington, were as high as $35.6 \mathrm{mg} / \mathrm{m}^{3}$ and averaged $13.3 \mathrm{mg} / \mathrm{m}^{3}$ during the first week following the May 18, 1980 eruption of Mount St. Helens (Merchant et al. 1982 [DIRS 160102], pp. 912 to 913). Five to $10 \mathrm{~mm}$ of ash were deposited at Yakima during that eruption (Sarna-Wojcicki et al. 1982 [DIRS 160227], Figure 336).

The peak, short-term (about 4-hour) TSP concentration measured in Missoula, Montana, on May 19 (the day of greatest ash fall at that location) was $19.9 \mathrm{mg} / \mathrm{m}^{3}$. The average daily concentrations there decreased from $11.1 \mathrm{mg} / \mathrm{m}^{3}$ on May 19 to $0.9 \mathrm{mg} / \mathrm{m}^{3}$ on May 22 
(Johnson et al. 1982 [DIRS 164149], pp. 1067 to 1068). Approximately 2.5 to $5 \mathrm{~mm}$ of ash was deposited at Missoula (Sarna-Wojcicki et al. 1982 [DIRS 160227], Figure 336).

The daily average TSP concentration in Clarkston, Washington, on May 18 was $0.68 \mathrm{mg} / \mathrm{m}^{3}$. Approximately $0.5 \mathrm{~mm}$ of ash was deposited there from that day's eruption. At Longview, Washington, the average daily concentration on May 27 was $1.42 \mathrm{mg} / \mathrm{m}^{3}$. Approximately 1 to 2 $\mathrm{mm}$ of ash was deposited on that city during an eruption on May 25 (DTN: MO0008SPATSP00.013 [DIRS 151750], EPA monitoring sites 53-003-0003 and 53-015-0008; Sarna-Wojcicki et al. 1982 [DIRS 160227], Figures 336 and 344).

Approximately 10 percent or less of the ash from Mount St. Helens was $\leq 10 \mu \mathrm{m}\left(\mathrm{PM}_{10}\right)$ (Craighead et al. 1983 [DIRS 160338], p. 6; Buist et al. 1986[DIRS 144632], p. 40). $\mathrm{PM}_{10}$ is defined as particulate matter having mass median aerodynamic diameter less than $10 \mu \mathrm{m}$.

Soufriere Hills-Peak $\mathrm{PM}_{10}$ concentrations at 3 locations during an eruption of the Soufriere Hills volcano (Montserrat, British West Indies) in 1997 were approximately 0.3 to $1.0 \mathrm{mg} / \mathrm{m}^{3}$ outside a school, $0.1 \mathrm{mg} / \mathrm{m}^{3}$ inside that school, and 0.4 to $1.5 \mathrm{mg} / \mathrm{m}^{3}$ outside a hotel (Baxter et al. 1999 [DIRS 150713], Figure 3 and p. 1142). The fine ashfall deposits from this volcano typically contained 60 to 70 percent (by weight) of 10 to $125 \mu \mathrm{m}$ particles and 13 to 20 percent of particles $<10 \mu \mathrm{m}$. Using a ratio of $\mathrm{PM}_{10}$ to TSP concentrations of 1:5 (calculated based on the average fraction of particles $<10 \mu \mathrm{m}$ in the deposited ash), peak TSP concentrations were approximately 1.5 to $5.0 \mathrm{mg} / \mathrm{m}^{3}, 0.5 \mathrm{mg} / \mathrm{m}^{3}$, and 2.0 to $7.5 \mathrm{mg} / \mathrm{m}^{3}$, respectively at the three locations. These concentrations likely did not include resuspended particles as they were taken late in the day after activities at the sites had ceased.

Searl et al. (2002 [DIRS 160104], Table 11) estimated mean personal $\mathrm{PM}_{10}$ exposure concentrations for various activity levels during and after eruptions of the Soufriere Hills volcano. Using a $\mathrm{PM}_{10}$ to TSP ratio of $1: 5$, estimated TSP concentrations during periods with alert levels to very high levels of ash were 1.5 to $5 \mathrm{mg} / \mathrm{m}^{3}$ for people inactive indoors, 2.5 to $10 \mathrm{mg} / \mathrm{m}^{3}$ for active indoors, 5 to $15 \mathrm{mg} / \mathrm{m}^{3}$ for active outside, and 25 to $50 \mathrm{mg} / \mathrm{m}^{3}$ for dusty occupations. These estimates include the influence of particle resuspension during activities.

Mt. Spurr-The maximum hourly $\mathrm{PM}_{10}$ concentration in Anchorage Alaska, during the 1992 eruption of Mt. Spurr was $3 \mathrm{mg} / \mathrm{m}^{3}$. The 24-hour average concentrations the day after the eruption was $0.565 \mathrm{mg} / \mathrm{m}^{3}$. Approximately 8 to 15 percent (by weight) of ash particles from that eruption collected near Anchorage were $<15 \mu \mathrm{m}$, and 5 to 10 percent were between 2.5 and 10 $\mu \mathrm{m}$, resulting in an approximate $\mathrm{PM}_{10}$ to TSP ratio of 1:10. Based on this ratio, the peak TSP concentration in Anchorage was about $30 \mathrm{mg} / \mathrm{m}^{3}$ and the 24-hour average was about $5.7 \mathrm{mg} / \mathrm{m}^{3}$ (Gordian et al. 1996 [DIRS 160111], p. 290; McGimsey et al. 2001 [DIRS 160386], Figures 11 and 12).

Mt. Sakurijima-Yano et al. (1990 [DIRS 160112], p. 373) stated that peak, 2-minute concentrations higher than $2 \mathrm{mg} / \mathrm{m}^{3}$ have been measured in high-exposure areas after eruptions of Mount Sakurijima (Japan), and that "these high levels of suspended particulate matter seldom last long, and they usually decrease rapidly to approximately $0.1 \mathrm{mg} / \mathrm{m}^{3}$." 
In summary, daily average concentrations of ash outdoors during an eruption may be as low or lower than $1 \mathrm{mg} / \mathrm{m}^{3}$ for light ashfall events or as high or higher than $15 \mathrm{mg} / \mathrm{m}^{3}$ for high ashfall events. Concentrations indoors would be much lower (see also BSC 2004 [DIRS 169458], Section 6.2). It should be noted that high ambient concentrations reported here do not result in an under-representation of the risk estimate because they likely are overestimates of concentrations inhaled because it is well documented that during volcanic eruptions people take protective actions, such as staying indoors and wearing masks, to reduce the amount of ash they inhale (Johnson et al. 1982 [DIRS 164149]; Buist et al. 1986 [DIRS 144632]; Nania et al. 1994 [DIRS 164156]).

\subsubsection{Development of Inhalation Dose Factors}

As noted before, inhalation dose factors are developed to account for the inhalation exposure during an eruption phase. The inhalation dose factors are developed separately from BDCFs because BDCFs are developed for annual exposures and do not address relatively short-term exposure conditions during a volcanic eruption. The biosphere model described in the Biosphere Model Report (BSC 2004 [DIRS 169460]) is not used to calculate the inhalation dose factors.

The inhalation dose factor for a given primary radionuclide is numerically equal to the dose resulting from a one-day intake of this radionuclide (and associated short-lived decay products, if present) by inhaling air containing a unit activity concentration of the radionuclide under consideration (e.g., $1 \mathrm{~Bq} / \mathrm{m}^{3}$ ) and its short-lived decay products. For a unit activity concentration of a radionuclide in air, radionuclide intake depends on inhalation exposure time and breathing rate.

In the biosphere model, the dose to the RMEI from inhalation exposure to a given primary radionuclide $i$ and its short-lived decay products present in airborne particulates is calculated (modified from annual dose to daily dose from BSC 2004 [DIRS 169460], Section 6.4.8.1) as

$$
D_{i n h, p, i}=E D C F_{i n h, i}\left[\sum_{n} C a_{i, n} B R_{n} \sum_{m}\left(P P_{m} t_{n, m}\right)\right]
$$

where

$\begin{array}{ll}D_{i n h, p, i}= & \text { daily dose from inhalation exposure to primary radionuclide } i \text { in } \\ & \text { resuspended particles }(\mathrm{Sv} / \mathrm{d}) \\ E D C F_{i n h, i}= & \text { effective dose coefficient for inhalation of primary radionuclide } i(\mathrm{~Sv} / \mathrm{Bq}) \\ n & \text { environment index; } n=1 \text { for active outdoors, } 2 \text { for inactive outdoors, } 3 \text { for } \\ & \text { active indoors, } 4 \text { for asleep indoors, and } 5 \text { for away from the contaminated } \\ & \text { area } \\ = & \text { activity concentration of primary radionuclide } i \text { in air for environment } n \\ C a_{i, n} & \left(\mathrm{~Bq} / \mathrm{m}^{3}\right) \\ B R_{n} & =\end{array}$




$$
t_{n, m}=\begin{aligned}
& \text { number of hours per day a population group } m \text { spends in environment } n \\
& (\mathrm{hr} / \mathrm{d}) .
\end{aligned}
$$

For calculating the inhalation dose, the exposure times for different population groups and environments, as well as the associated breathing rates, were developed (see Section 4.1 for a list of model input parameters). For the duration of volcanic eruption, environment-specific breathing rates and the fractions of time spent indoors versus outdoors are considered relatively unchanged compared to the pre-eruption conditions. Therefore, the same parameter values for lifestyle characteristics as those used for the BDCF calculation were used to develop inhalation dose factors for the eruption phase.

Because a volcanic eruption is an unusual event, it is possible that people would not behave as they would under normal circumstances. However, it is difficult to predict how the human behavior would change. Some people may seek shelter from falling ash and spend more time indoors where exposure would be reduced, while other people may, for instance, perform ash removal from their property and spend more time outdoors.

Ash depths $18 \mathrm{~km}$ downwind from Yucca Mountain, for the wind blowing to the south, were predicted to range from 0.07 to $55 \mathrm{~cm}$, based on 100 realizations of the ASHPLUME model (BSC 2004 [DIRS 170026], Table 6-4). Approximately 35 percent of the predicted depths of deposited ash were less than $1 \mathrm{~cm}, 75$ percent were less than $5 \mathrm{~cm}$, and 90 percent were less than $15 \mathrm{~cm}$ (BSC 2004 [DIRS 170026], Table 6-4). Ash depths at the location of the RMEI (18 km south of Yucca Mountain) would be about 2 orders of magnitude or more lower under normal, variable wind conditions (CRWMS M\&O 2000 [DIRS 153246], Section 3.10.5.1 and Figure 3.10-14) because the wind at Yucca Mountain blows to the south infrequently (BSC 2004 [DIRS 170026], Figure 8-1).

For the small amount of ashfall and an eruption lasting for several days, it is likely that the airborne particulate concentrations would not be substantially different from the pre-eruption levels. If this is the case, people would not modify their behavior to the extent that their overall average daily breathing rate would be affected, in which case the assumption is realistic. If airborne particulate concentrations were much greater than the pre-eruption levels, people would take actions to reduce the amount of ash they inhale, such as staying indoors and wearing masks (Section 6.3.1), and this assumption would lead to conservative results. In summary, using the same lifestyle characteristics parameter values for the eruption phase inhalation dose factors as those used for the volcanic ash exposure scenario BDCF calculations is considered technically defensible and not to result in an under-representation of the risk estimate.

The ERMYN divides the biosphere into the five environments (BSC 2005 [DIRS 172827], Section 6.2). These mutually exclusive environments represent the behavioral and environmental combinations for which a person may receive a substantially different rate of exposure via inhalation or external exposure. These environments are:

Away from Potentially Contaminated Area-This category encompasses the region away from areas contaminated by groundwater or volcanic ash. Time spent away from contaminated area includes time spent working and commuting to work by those that work outside of contaminated areas. 
Active Outdoors - This category encompasses those locations within contaminated area where people actively disturb soil surface thus increasing particulate and contaminant concentration in air. Time spent active outdoors includes time spent outdoors in contaminated areas conducting activities that resuspend soil.

Inactive Outdoors - This category represents outdoor locations within contaminated areas not associated with soil-surface disturbing activities. In this environment people spend time commuting and conducting activities that do not resuspend soil.

Asleep Indoors-This category represents indoor locations within contaminated areas where people spend time sleeping.

Active Indoors - This category represents indoor locations within contaminated areas where people spend time awake, including work time. In the model, time spent in this environment is calculated as the remainder of the day not spent in the other environments.

Two environments that are not associated with soil disturbing activities are the inactive outdoor and asleep indoor environments. The modes of the mass loading distributions are $0.060 \mathrm{mg} / \mathrm{m}^{3}$ and $0.030 \mathrm{mg} / \mathrm{m}^{3}$ for the inactive outdoor and asleep indoor environments, respectively (DTN: MO0407SPAINEXI.002 [DIRS 170597]), indicating that dwellings provide about 50 percent reduction of the outdoor mass loading level under the conditions of no soil disturbance.

It is postulated that during volcanic eruption, the average level of mass loading indoors arising from the original deposition of tephra would be less than that for the outdoor environment. This assumption does not pertain to the concentrations of particulates in air that were resuspended due to atmospheric or mechanical processes following the initial deposition.

Therefore, the indoor reduction factor can be defined as the ratio of the post-volcanic mass loading values indoor and outdoor in the absence of soil disturbing activities causing resuspension of deposited ash. Undisturbed conditions were chosen because the process of resuspension and the resulting inhalation of resuspended particulates are included in the BDCFs.

Thus, assuming that the radionuclide concentration in the indoor air is a fraction of that in the outdoor air, Equation 6.3-1 can be modified as

$$
\begin{aligned}
D_{\text {inh }, p, i} & =E D C F_{\text {inh }, i}\left[\sum_{n} C a_{i, \text { outdoor }} I R F_{n} B R_{n} \sum_{m}\left(P P_{m} t_{n, m}\right)\right] \\
& =C a_{i, \text { outdoor }} E D C F_{\text {inh }, i}\left[\sum_{n} I R F_{n} B R_{n} \sum_{m}\left(P P_{m} t_{n, m}\right)\right] \\
& =C a_{i, \text { outdoor }} D F_{i}
\end{aligned}
$$

where

$$
\begin{aligned}
& C a_{i, \text { outdoor }}= \text { activity concentration of a radionuclide } i \text { in outdoor air for the ash that } \\
& \text { has not yet fallen on the ground }\left(\mathrm{Bq} / \mathrm{m}^{3}\right)
\end{aligned}
$$


$I R F_{n} \quad=\quad$ indoor reduction factor for activity concentration in air (dimensionless)

$D F_{i} \quad=\quad$ inhalation dose factor for a primary radionuclide $i\left(\mathrm{~Sv} / \mathrm{d}\right.$ per $\left.\mathrm{Bq} / \mathrm{m}^{3}\right)$.

The activity concentration of a radionuclide $\mathrm{i}$ in outdoor air for the ash that has not yet fallen on the ground, $\mathrm{Ca}_{i, \text { outdoor }}$, does not depend on the outdoor environment, as defined for the biosphere model (i.e., active outdoor and inactive outdoor), because this quantity is independent of human activities. The indoor reduction factor is equal to 1 for the outdoor environments (active outdoors and inactive outdoors) and to 0.5 for the indoor environments (asleep indoors and active indoors). The term in the brackets in Equation 6.3-2 is the effective daily breathing rate, i.e., the volume of outdoor air that contains the same amount of contaminant (radionuclide) as the air that is breathed in by a person in one day.

An indoor reduction factor of 0.5 is considered conservative since measurements of indoor to outdoor air concentration ratios during volcanic eruptions were found to be less than 0.5 (Baxter et al. 1999 [DIRS 150713], Figure 3 and p. 1142). However, since the inhalation dose factors are likely to be used as a screening tool, it is appropriate to use more conservative values so as not to underestimate potential dose.

The inhalation dose factor in Equation 6.3-2 is expressed as

$$
D F_{i}=E D C F_{i n h, i}\left[\sum_{n} I R F_{n} B R_{n} \sum_{m}\left(P P_{m} t_{n, m}\right)\right]
$$

The effective dose coefficients for inhalation include, where applicable, contributions from the associated short-lived decay products. They were developed by calculating a sum of the dose coefficients for inhalation, weighted by the associated branching fractions, as shown in Table 6.3-1 for the ICRP 30-based dose coefficients obtained from FGR 11 (Eckerman et al. 1988 [DIRS 101069]) (DTN: MO0407SPACRBSM.002 [DIRS 170677]). These dose coefficients were developed for radiation protection in the workplace and are usually applied to chronic low-dose, low-dose rate exposures. However, the same values are also recommended for conducting radiological assessments for consequence analysis in the case of accidental releases (Sjoreen et al. 2001 [DIRS 164093], Section 4.9.1), where calculated doses can exceed 250 rem (Sjoreen et al. 2001 [DIRS 164093], Table 7.1). ICRP 72 dose coefficients for inhalation and the associated effective dose coefficients are listed in Table 6.3-2.

The inhalation dose factors are calculated as deterministic quantities using the mean values of parameters. They are provided to allow estimating the dose during the eruption phase. In the previous performance assessment, the dose calculated for the eruptive phase did not significantly contribute to the calculated expected annual dose, which was shown in the sensitivity analyses for the TSPA for the Site Recommendation (CRWMS M\&O 2000 [DIRS 153246], Sections 3.10.3.1 and 5.2.9.9). Calculations of the individual terms in Equation 6.3-3 are shown in Table 6.3-3. 
Table 6.3-1. Effective Dose Coefficients for Inhalation Consistent with ICRP 30 Methods

\begin{tabular}{|c|c|c|c|}
\hline Primary Radionuclide & $\begin{array}{c}\text { Decay Product } \\
\text { (branching fraction if not } 100 \% \text {, } \\
\text { half-life) }\end{array}$ & $\begin{array}{l}\text { Dose Coefficient } \\
\text { (Sv/Bq) }\end{array}$ & $\begin{array}{c}\text { Effective Dose } \\
\text { Coefficient (Sv/Bq) }\end{array}$ \\
\hline $\mathrm{Sr}-90+\mathrm{D}^{\mathrm{a}}$ & Y-90 $(64.0 \mathrm{hr})$ & $\begin{array}{l}6.47 \mathrm{E}-08 \\
2.28 \mathrm{E}-09\end{array}$ & $6.70 \mathrm{E}-08$ \\
\hline Tc-99 & - & 2.25E-09 & 2.25E-09 \\
\hline$S n-126+D$ & $\begin{array}{l}\text { Sb-126m (19.0 min }) \\
\text { Sb-126 (14\%, } 12.4 \text { day })\end{array}$ & $\begin{array}{l}2.69 \mathrm{E}-08 \\
9.17 \mathrm{E}-12 \\
3.17 \mathrm{E}-09\end{array}$ & 2.74E-08 \\
\hline Cs-137+D & $\mathrm{Ba}-137 \mathrm{~m}(94.6 \%, 2.552 \mathrm{~min})$ & $\begin{array}{l}8.63 \mathrm{E}-09 \\
0.00 \mathrm{E}+00 \\
\end{array}$ & 8.63E-09 \\
\hline \multicolumn{4}{|c|}{ Thorium Series (4n) } \\
\hline Pu-240 & - & $1.16 \mathrm{E}-04$ & $1.16 \mathrm{E}-04$ \\
\hline $\mathrm{U}-236$ & - & 3.39E-05 & $3.39 \mathrm{E}-05$ \\
\hline Th-232 & - & $4.43 E-04$ & 4.43E-04 \\
\hline $\mathrm{Ra}-228+\mathrm{D}^{\mathrm{b}}$ & Ac-228 $(6.13 \mathrm{hr})$ & $\begin{array}{l}1.29 \mathrm{E}-06 \\
8.33 \mathrm{E}-08\end{array}$ & 1.37E-06 \\
\hline U-232 & - & $1.78 \mathrm{E}-04$ & $1.78 \mathrm{E}-04$ \\
\hline Th-228+D & $\begin{array}{l}\text { Ra-224 (3.66 d) } \\
\text { Rn-220 (55.6 s) } \\
\text { Po-216 (0.15 s) } \\
\text { Pb-212 }(10.64 \mathrm{hr}) \\
\text { Bi-212 }(60.55 \mathrm{~min}) \\
\text { Po-212 }(64.07 \%, 0.305 \mu \mathrm{s}) \\
\text { TI-208 }(35.93 \%, 3.07 \mathrm{~min})\end{array}$ & $\begin{array}{l}9.23 \mathrm{E}-05 \\
8.53 \mathrm{E}-07 \\
0.00 \mathrm{E}+00 \\
0.00 \mathrm{E}+00 \\
4.56 \mathrm{E}-08 \\
5.83 \mathrm{E}-09 \\
0.00 \mathrm{E}+00 \\
0.00 \mathrm{E}+00\end{array}$ & $9.32 \mathrm{E}-05$ \\
\hline \multicolumn{4}{|c|}{ Neptunium Series $(4 n+1)$} \\
\hline Am-241 & - & $1.20 \mathrm{E}-04$ & 1.20E-04 \\
\hline $\mathrm{Np}-237+\mathrm{D}$ & $\mathrm{Pa}-233(27.0 \mathrm{~d})$ & $\begin{array}{l}1.46 \mathrm{E}-04 \\
2.58 \mathrm{E}-09\end{array}$ & $1.46 \mathrm{E}-04$ \\
\hline $\mathrm{U}-233$ & - & $3.66 \mathrm{E}-05$ & $3.66 \mathrm{E}-05$ \\
\hline Th-229+D & $\begin{array}{l}\text { Ra-225 (14.8 d) } \\
\text { Ac-225 (10.0 d) } \\
\text { Fr-221 (4.8 min) } \\
\text { At-217 (32.3 ms) } \\
\text { Bi-213 (45.65 min }) \\
\text { Po-213 (97.84\%, } 4.2 \mu \mathrm{s}) \\
\text { Tl-209 }(2.16 \%, 2.2 \mathrm{~min}) \\
\text { Pb-209 (3.253 hr) }\end{array}$ & $\begin{array}{l}5.80 E-04 \\
2.10 E-06 \\
2.92 E-06 \\
0.00 E+00 \\
0.00 E+00 \\
4.63 E-09 \\
0.00 E+00 \\
0.00 E+00 \\
2.56 E-11\end{array}$ & $5.85 E-04$ \\
\hline
\end{tabular}


Table 6.3-1. Effective Dose Coefficients for Inhalation Consistent with ICRP 30 Methods (Continued)

\begin{tabular}{|c|c|c|c|}
\hline Primary Radionuclide & $\begin{array}{c}\text { Decay Product } \\
\text { (branching fraction if not } 100 \% \text {, } \\
\text { half-life) }\end{array}$ & $\begin{array}{c}\text { Dose Coefficient } \\
(\mathrm{Sv} / \mathrm{Bq})\end{array}$ & $\begin{array}{c}\text { Effective Dose } \\
\text { Coefficient (Sv/Bq) }\end{array}$ \\
\hline \multicolumn{4}{|c|}{ UraniumSeries $(4 n+2)$} \\
\hline Pu-242 & - & 1.11E-04 & 1.11E-04 \\
\hline$U-238+D$ & $\begin{array}{l}\text { Th-234 }(24.10 \mathrm{~d}) \\
\mathrm{Pa}-234 \mathrm{~m}(99.80 \%, 1.17 \mathrm{~min}) \\
\text { Pa-234 }(0.33 \%, 6.7 \mathrm{hr})\end{array}$ & $\begin{array}{l}3.20 \mathrm{E}-05 \\
9.47 \mathrm{E}-09 \\
0.00 \mathrm{E}+00 \\
2.20 \mathrm{E}-10 \\
\end{array}$ & $3.20 \mathrm{E}-05$ \\
\hline Pu-238 & - & 1.06E-04 & 1.06E-04 \\
\hline $\mathrm{U}-234$ & - & $3.58 E-05$ & $3.58 \mathrm{E}-05$ \\
\hline Th-230 & - & $8.80 \mathrm{E}-05$ & $8.80 \mathrm{E}-05$ \\
\hline $\mathrm{Ra}-226+\mathrm{D}$ & $\begin{array}{l}\text { Rn-222 }(3.8235 \mathrm{~d}) \\
\text { Po-218 }(3.05 \mathrm{~min}) \\
\text { Pb-214 }(99.98 \%, 26.8 \mathrm{~min}) \\
\text { At-218 }(0.02 \%, 2 \mathrm{~s}) \\
\text { Bi-214 }(19.9 \mathrm{~min}) \\
\text { Po-214 }\left(99.98 \%, 1.64 \times 10^{-4} \mathrm{~s}\right) \\
\text { TI-210 (0.02\%, } 1.3 \mathrm{~min})\end{array}$ & $\begin{array}{c}2.32 \mathrm{E}-06 \\
0.00 \mathrm{E}+00 \\
0.00 \mathrm{E}+00 \\
2.11 \mathrm{E}-09 \\
0.00 \mathrm{E}+00 \\
1.78 \mathrm{E}-09 \\
0.00 \mathrm{E}+00 \\
0.00 \mathrm{E}+00\end{array}$ & $2.32 \mathrm{E}-06$ \\
\hline $\mathrm{Pb}-210+\mathrm{D}$ & $\begin{array}{l}\text { Bi-210 }(5.012 d) \\
\text { Po-210 }(138.38 d)\end{array}$ & $\begin{array}{l}3.67 \mathrm{E}-06 \\
5.29 \mathrm{E}-08 \\
2.54 \mathrm{E}-06\end{array}$ & 6.26E-06 \\
\hline \multicolumn{4}{|c|}{ Actinium Series $(4 n+3)$} \\
\hline$A m-243+D$ & $\mathrm{~Np}-239(2.355 \mathrm{~d})$ & $\begin{array}{l}1.19 \mathrm{E}-04 \\
6.78 \mathrm{E}-10 \\
\end{array}$ & $1.19 \mathrm{E}-04$ \\
\hline Pu-239 & - & $1.16 \mathrm{E}-04$ & $1.16 \mathrm{E}-04$ \\
\hline $\mathrm{U}-235+\mathrm{D}$ & Th-231 (25.52 hr) & $\begin{array}{l}3.32 E-05 \\
2.37 E-10\end{array}$ & $3.32 \mathrm{E}-05$ \\
\hline Pa-231 & - & 3.47E-04 & 3.47E-04 \\
\hline$A c-227+D$ & $\begin{array}{l}\text { Th-227 }(98.62 \%, 18.718 \mathrm{~d}) \\
\text { Fr-223 }(1.38 \%, 21.8 \mathrm{~min}) \\
\operatorname{Ra}-223(11.434 \mathrm{~d}) \\
\operatorname{Rn}-219(3.96 \mathrm{~s}) \\
\text { Po-215 }(1.78 \mathrm{~ms}) \\
\text { Pb-211 }(36.1 \mathrm{~min}) \\
\text { Bi-211 }(2.15 \mathrm{~min}) \\
\text { Tl-207 (99.72\%, } 4.77 \mathrm{~min}) \\
\text { Po-211 (0.28\%, 0.516 s) }\end{array}$ & $\begin{array}{l}1.81 \mathrm{E}-03 \\
4.37 \mathrm{E}-06 \\
1.68 \mathrm{E}-09 \\
2.12 \mathrm{E}-06 \\
0.00 \mathrm{E}+00 \\
0.00 \mathrm{E}+00 \\
2.35 \mathrm{E}-09 \\
0.00 \mathrm{E}+00 \\
0.00 \mathrm{E}+00 \\
0.00 \mathrm{E}+00\end{array}$ & $1.82 E-03$ \\
\hline
\end{tabular}

Source: DTN: MO0407SPACRBSM.002 [DIRS 170677].

Notes: "a " $D$ " denotes that the radionuclide is treated together with the short-lived (half-life $<180$ days) decay product.

${ }^{6}$ Indented radionuclides are long-lived decay products considered separately from the parents.

${ }^{c}$ Calculated as a sum of dose coefficients for primary radionuclides and the short-lived decay products, weighted by the branching fractions, where applicable. 
Table 6.3-2. Effective Dose Coefficients for Inhalation Consistent with ICRP 72 Methods

\begin{tabular}{|c|c|c|c|}
\hline Primary Radionuclide & $\begin{array}{c}\text { Decay Product } \\
\text { (branching fraction if not } 100 \% \text {, } \\
\text { half-life) }\end{array}$ & $\begin{array}{c}\text { Dose Coefficient } \\
\text { (Sv/Bq) }\end{array}$ & $\begin{array}{c}\text { Effective Dose } \\
\text { Coefficient (Sv/Bq) }\end{array}$ \\
\hline $\mathrm{Sr}-90+\mathrm{D}^{\mathrm{a}}$ & $\mathrm{Y}-90(64.0 \mathrm{hr})$ & $\begin{array}{l}1.6 \mathrm{E}-07 \\
1.5 \mathrm{E}-09 \\
\end{array}$ & $1.62 \mathrm{E}-07$ \\
\hline Tc-99 & - & $1.3 \mathrm{E}-08$ & $1.3 \mathrm{E}-08$ \\
\hline$S n-126+D$ & $\begin{array}{l}\text { Sb-126m (19.0 min) } \\
\text { Sb-126 (14\%, } 12.4 \text { day })\end{array}$ & $\begin{array}{l}2.8 \mathrm{E}-08 \\
2.0 \mathrm{E}-11 \\
3.2 \mathrm{E}-09 \\
\end{array}$ & $2.85 \mathrm{E}-08$ \\
\hline Cs-137+D & $\mathrm{Ba}-137 \mathrm{~m}(94.6 \%, 2.552 \mathrm{~min})$ & $\begin{array}{l}3.9 \mathrm{E}-08 \\
0.0 \mathrm{E}+00\end{array}$ & $3.9 \mathrm{E}-08$ \\
\hline \multicolumn{4}{|c|}{ Thorium Series (4n) } \\
\hline Pu-240 & - & $1.2 \mathrm{E}-04$ & $1.2 \mathrm{E}-04$ \\
\hline U-236 & - & $8.7 \mathrm{E}-06$ & 8.7E-06 \\
\hline Th-232 & - & $1.1 \mathrm{E}-04$ & $1.1 \mathrm{E}-04$ \\
\hline $\mathrm{Ra}-228+D^{b}$ & Ac-228 $(6.13 \mathrm{hr})$ & $\begin{array}{l}1.6 \mathrm{E}-05 \\
2.5 \mathrm{E}-08\end{array}$ & $1.6 \mathrm{E}-05$ \\
\hline $\mathrm{U}-232$ & - & $3.7 \mathrm{E}-05$ & $3.7 \mathrm{E}-05$ \\
\hline Th-228+D & $\begin{array}{l}\text { Ra-224 (3.66 d) } \\
\text { Rn-220 (55.6 s) } \\
\text { Po-216 (0.15 s) } \\
\text { Pb-212 (10.64 hr) } \\
\text { Bi-212 }(60.55 \mathrm{~min}) \\
\text { Po-212 }(64.07 \%, 0.305 \mu \mathrm{s}) \\
\text { Tl-208 }(35.93 \%, 3.07 \mathrm{~min})\end{array}$ & $\begin{array}{l}4.0 \mathrm{E}-05 \\
3.4 \mathrm{E}-06 \\
0.0 \mathrm{E}+00 \\
0.0 \mathrm{E}+00 \\
1.9 \mathrm{E}-07 \\
3.1 \mathrm{E}-08 \\
0.0 \mathrm{E}+00 \\
0.0 \mathrm{E}+00\end{array}$ & $4.36 \mathrm{E}-05$ \\
\hline \multicolumn{4}{|c|}{ Neptunium Series $(4 n+1)$} \\
\hline $\mathrm{Am}-241$ & - & $9.6 \mathrm{E}-05$ & $9.6 \mathrm{E}-05$ \\
\hline $\mathrm{Np}-237+\mathrm{D}$ & $\mathrm{Pa}-233$ (27.0 d) & $\begin{array}{l}5.0 \mathrm{E}-05 \\
3.9 \mathrm{E}-09 \\
\end{array}$ & $5.0 \mathrm{E}-05$ \\
\hline U-233 & - & $9.6 \mathrm{E}-06$ & $9.6 \mathrm{E}-06$ \\
\hline Th-229+D & $\begin{array}{l}\operatorname{Ra}-225(14.8 \mathrm{~d}) \\
\mathrm{Ac}-225(10.0 \mathrm{~d}) \\
\mathrm{Fr}-221(4.8 \mathrm{~min}) \\
\mathrm{At}-217(32.3 \mathrm{~ms}) \\
\mathrm{Bi}-213(45.65 \mathrm{~min}) \\
\mathrm{Po}-213(97.84 \%, 4.2 \mu \mathrm{s}) \\
\mathrm{Tl}-209(2.16 \%, 2.2 \mathrm{~min}) \\
\mathrm{Pb}-209(3.253 \mathrm{hr})\end{array}$ & $\begin{array}{l}2.4 \mathrm{E}-04 \\
7.7 \mathrm{E}-06 \\
8.5 \mathrm{E}-06 \\
0.0 \mathrm{E}+00 \\
0.0 \mathrm{E}+00 \\
3.0 \mathrm{E}-08 \\
0.0 \mathrm{E}+00 \\
0.0 \mathrm{E}+00 \\
6.1 \mathrm{E}-11\end{array}$ & $2.56 \mathrm{E}-04$ \\
\hline
\end{tabular}


Table 6.3-2. Effective Dose Coefficients for Inhalation Consistent with ICRP 72 Methods (Continued)

\begin{tabular}{|c|c|c|c|}
\hline Primary Radionuclide & $\begin{array}{c}\text { Decay Product } \\
\text { (branching fraction if not } 100 \% \text {, } \\
\text { half-life) }\end{array}$ & $\begin{array}{c}\text { Dose Coefficient } \\
\text { (Sv/Bq) }\end{array}$ & $\begin{array}{c}\text { Effective Dose } \\
\text { Coefficient (Sv/Bq) } \\
\end{array}$ \\
\hline \multicolumn{4}{|c|}{ Uranium Series $(4 n+2)$} \\
\hline Pu-242 & - & $1.1 \mathrm{E}-04$ & $1.1 \mathrm{E}-04$ \\
\hline$U-238+D$ & $\begin{array}{l}\text { Th-234 }(24.10 \mathrm{~d}) \\
\text { Pa-234m }(99.80 \%, 1.17 \mathrm{~min}) \\
\text { Pa-234 }(0.33 \%, 6.7 \mathrm{hr})\end{array}$ & $\begin{array}{l}8.0 \mathrm{E}-06 \\
7.7 \mathrm{E}-09 \\
0.0 \mathrm{E}+00 \\
4.0 \mathrm{E}-10 \\
\end{array}$ & 8.01E-06 \\
\hline Pu-238 & - & $1.1 \mathrm{E}-04$ & $1.1 \mathrm{E}-04$ \\
\hline U-234 & - & 9.4E-06 & $9.4 \mathrm{E}-06$ \\
\hline Th-230 & - & $1.0 \mathrm{E}-04$ & $1.0 \mathrm{E}-04$ \\
\hline $\mathrm{Ra}-226+\mathrm{D}$ & $\begin{array}{l}\text { Rn-222 }(3.8235 \mathrm{~d}) \\
\text { Po-218 }(3.05 \mathrm{~min}) \\
\text { Pb-214 }(99.98 \%, 26.8 \mathrm{~min}) \\
\text { At-218 }(0.02 \%, 2 \mathrm{~s}) \\
\text { Bi-214 }(19.9 \mathrm{~min}) \\
\text { Po-214 }\left(99.98 \%, 1.64 \times 10^{-4} \mathrm{~s}\right) \\
\text { Tl-210 }(0.02 \%, 1.3 \mathrm{~min})\end{array}$ & $\begin{array}{l}9.5 \mathrm{E}-06 \\
0.0 \mathrm{E}+00 \\
0.0 \mathrm{E}+00 \\
1.5 \mathrm{E}-08 \\
0.0 \mathrm{E}+00 \\
1.4 \mathrm{E}-08 \\
0.0 \mathrm{E}+00 \\
0.0 \mathrm{E}+00 \\
\end{array}$ & 9.53E-06 \\
\hline $\mathrm{Pb}-210+\mathrm{D}$ & $\begin{array}{l}\text { Bi-210 }(5.012 \mathrm{~d}) \\
\text { Po-210 }(138.38 \mathrm{~d})\end{array}$ & $\begin{array}{l}5.6 \mathrm{E}-06 \\
9.3 \mathrm{E}-08 \\
4.3 \mathrm{E}-06 \\
\end{array}$ & 9.99E-06 \\
\hline \multicolumn{4}{|c|}{ Actinium Series $(4 n+3)$} \\
\hline$A m-243+D$ & $\mathrm{~Np}-239(2.355 \mathrm{~d})$ & $\begin{array}{l}9.6 \mathrm{E}-05 \\
1.0 \mathrm{E}-09 \\
\end{array}$ & 19.6E-05 \\
\hline Pu-239 & - & $1.2 \mathrm{E}-04$ & $1.2 \mathrm{E}-04$ \\
\hline$U-235+D$ & Th-231 (25.52 hr) & $\begin{array}{l}8.5 \mathrm{E}-06 \\
3.3 \mathrm{E}-10\end{array}$ & $8.5 \mathrm{E}-06$ \\
\hline Pa-231 & - & $1.4 \mathrm{E}-04$ & $1.4 \mathrm{E}-04$ \\
\hline$A C-227+D$ & $\begin{array}{l}\text { Th-227 }(98.62 \%, 18.718 \mathrm{~d}) \\
\text { Fr-223 }(1.38 \%, 21.8 \mathrm{~min}) \\
\text { Ra-223 }(11.434 \mathrm{~d}) \\
\text { Rn-219 }(3.96 \mathrm{~s}) \\
\text { Po-215 }(1.78 \mathrm{~ms}) \\
\text { Pb-211 }(36.1 \mathrm{~min}) \\
\text { Bi-211 }(2.15 \mathrm{~min}) \\
\text { TI-207 }(99.72 \%, 4.77 \mathrm{~min}) \\
\text { Po-211 (0.28\%, 0.516 s) }\end{array}$ & $\begin{array}{c}5.5 E-04 \\
1.0 E-05 \\
8.9 E-10 \\
8.7 E-06 \\
0.0 E+00 \\
0.0 E+00 \\
1.2 E-08 \\
0.0 E+00 \\
0.0 E+00 \\
0.0 E+00\end{array}$ & $5.69 \mathrm{E}-04$ \\
\hline
\end{tabular}

Source: DTN: MO0503SPADCESR.000 [DIRS 172896].

Notes: a " $D$ " denotes that the radionuclide is treated together with the short-lived (half-life $<180$ days) decay product.

${ }^{\mathrm{b}}$ Indented radionuclides are long-lived decay products considered separately from the parents.

${ }^{c}$ Calculated as a sum of dose coefficients for primary radionuclides and the short-lived decay products, weighted by the branching fractions, where applicable. 


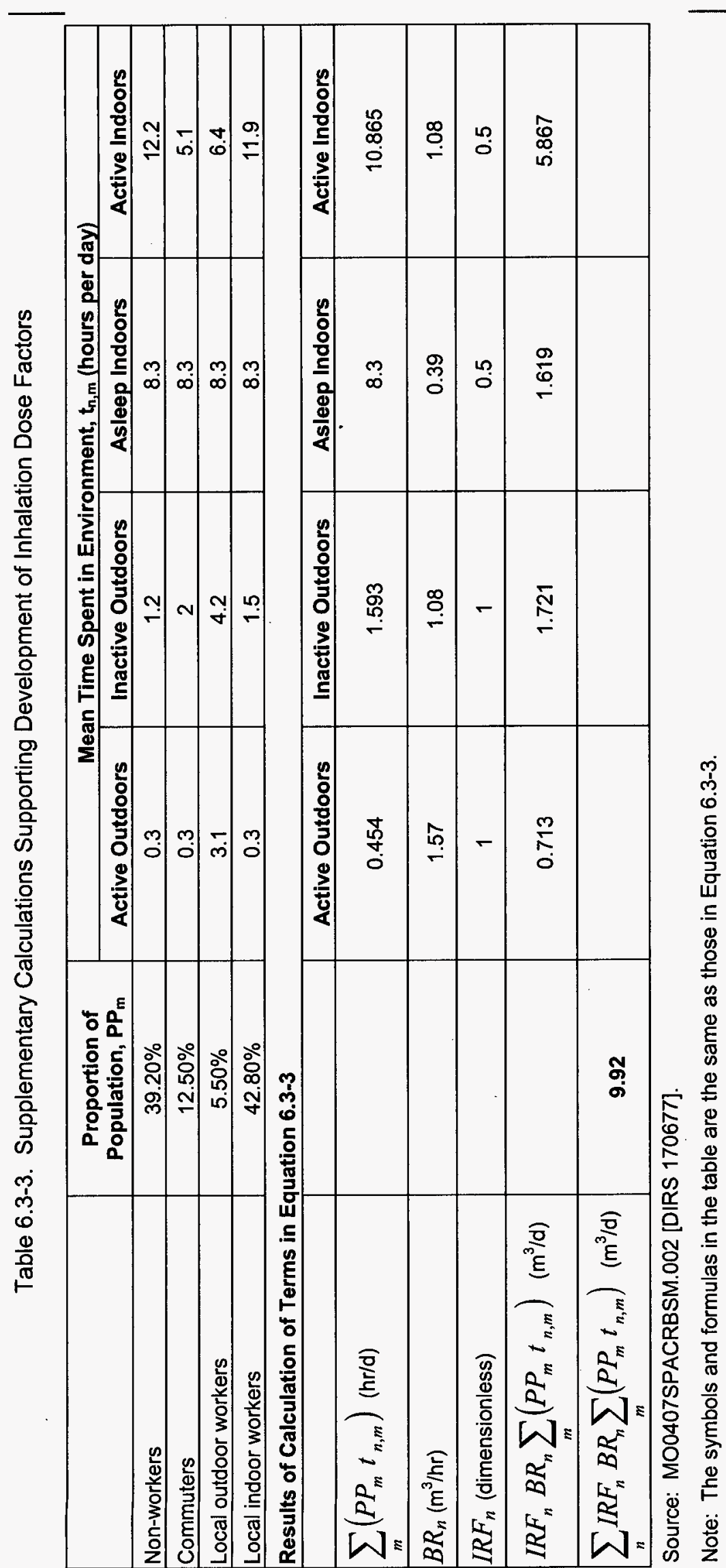


The inhalation dose factors based on the ICRP 30 dosimetric methods for radionuclides of interest for the volcanic ash exposure scenario, in units of $\mathrm{Sv} / \mathrm{d} \mathrm{per} \mathrm{Bq} / \mathrm{m}^{3}$ and $\mathrm{rem} / \mathrm{d} \mathrm{per} \mathrm{pCi} / \mathrm{m}^{3}$, calculated using Equation 6.3-3, are summarized in Table 6.3-4. Calculations were performed in an Excel spreadsheet Dose Factor Calculations_Rev 3.xls (Appendix B).

Table 6.3-4. ICRP 30-Based Inhalation Dose Factors for Eruptive Phase of the Volcanic Scenario

\begin{tabular}{|c|c|c|c|}
\hline \multirow[b]{2}{*}{ Radionuclide } & \multirow{2}{*}{$\begin{array}{c}\text { Effective Dose } \\
\text { Coefficient (Sv/Bq) } \\
\end{array}$} & \multicolumn{2}{|c|}{ Inhalation Dose Factor } \\
\hline & & Sv/d per Bq/m ${ }^{3}$ b & rem/d per $\mathrm{pCi} / \mathrm{m}^{3}$ \\
\hline${ }^{90} \mathrm{Sr}$ & $6.70 \mathrm{E}-08$ & $6.65 \mathrm{E}-07$ & 2.46E-06 \\
\hline${ }^{99} \mathrm{Tc}$ & 2.25E-09 & 2.23E-08 & 8.26E-08 \\
\hline${ }^{126} \mathrm{Sn}$ & 2.74E-08 & 2.72E-07 & 1.01E-06 \\
\hline${ }^{137} \mathrm{Cs}$ & 8.63E-09 & 8.56E-08 & 3.17E-07 \\
\hline${ }^{210} \mathrm{~Pb}$ & $6.26 \mathrm{E}-06$ & $6.21 \mathrm{E}-05$ & 2.30E-04 \\
\hline${ }^{226} \mathrm{Ra}$ & 2.32E-06 & 2.30E-05 & 8.51E-05 \\
\hline${ }^{227} \mathrm{Ac}$ & $1.82 E-03$ & 1.81E-02 & $6.68 \mathrm{E}-02$ \\
\hline${ }^{229} \mathrm{Th}$ & $5.85 \mathrm{E}-04$ & $5.80 \mathrm{E}-03$ & $2.15 \mathrm{E}-02$ \\
\hline${ }^{230} \mathrm{Th}$ & 8.80E-05 & 8.73E-04 & $3.23 E-03$ \\
\hline${ }^{232} \mathrm{Th}+{ }^{228} \mathrm{Ra}+{ }^{228} \mathrm{Th}$ & $5.38 E-04$ & 5.33E-03 & 1.97E-02 \\
\hline${ }^{231} \mathrm{~Pa}$ & 3.47E-04 & 3.44E-03 & 1.27E-02 \\
\hline${ }^{232} \mathrm{U}+{ }^{228} \mathrm{Th}$ & 2.71E-04 & 2.69E-03 & $9.95 \mathrm{E}-03$ \\
\hline${ }^{233} \mathrm{U}$ & $3.66 \mathrm{E}-05$ & 3.63E-04 & $1.34 \mathrm{E}-03$ \\
\hline${ }^{234} \mathrm{U}$ & $3.58 \mathrm{E}-05$ & 3.55E-04 & 1.31E-03 \\
\hline${ }^{236} \mathrm{U}$ & 3.39E-05 & $3.36 \mathrm{E}-04$ & $1.24 \mathrm{E}-03$ \\
\hline${ }^{238} \mathrm{U}$ & $3.20 \mathrm{E}-05$ & 3.17E-04 & 1.17E-03 \\
\hline${ }^{237} \mathrm{~Np}$ & 1.46E-04 & 1.45E-03 & 5.36E-03 \\
\hline${ }^{238} \mathrm{Pu}$ & $1.06 \mathrm{E}-04$ & $1.05 \mathrm{E}-03$ & 3.89E-03 \\
\hline${ }^{239} \mathrm{Pu}$ & $1.16 \mathrm{E}-04$ & 1.15E-03 & 4.26E-03 \\
\hline${ }^{240} \mathrm{Pu}$ & 1.16E-04 & 1.15E-03 & $4.26 \mathrm{E}-03$ \\
\hline${ }^{242} \mathrm{Pu}$ & 1.11E-04 & 1.10E-03 & 4.07E-03 \\
\hline${ }^{241} \mathrm{Am}$ & 1.20E-04 & 1.19E-03 & 4.40E-03 \\
\hline${ }^{243} \mathrm{Am}$ & 1.19E-04 & 1.18E-03 & 4.37E-03 \\
\hline
\end{tabular}

Source: The calculations are shown in Excel spreadsheet Dose Factor Calculations_Rev 3.x/s (Appendix B).

Notes: $\quad{ }^{a}$ From Table 6.3-1.

${ }^{b}$ Calculated by multiplying the effective dose coefficient for inhalation by the term calculated in Table 6.3-3 $\left(9.92 \mathrm{~m}^{3} / \mathrm{d}\right)$.

${ }^{c}$ Can be obtained from the values in the preceding column by multiplying them by unit conversion of 3.7 $(100 \mathrm{rem} / \mathrm{Sv} * 0.037 \mathrm{~Bq} / \mathrm{pCi})$. 
The inhalation dose factors based on the ICRP 72 dosimetric methods for radionuclides of interest for the volcanic ash exposure scenario, in units of Sv/d per $\mathrm{Bq} / \mathrm{m}^{3}$ and rem $/ \mathrm{d}$ per pCi $/ \mathrm{m}^{3}$, calculated using Equation 6.3-3, are summarized in Table 6.3-5. Calculations were performed in an Excel spreadsheet Dose Factor Calculations_Rev 4.xls (Appendix B).

Table 6.3-5. ICRP 72-Based Inhalation Dose Factors for Eruptive Phase of the Volcanic Scenario

\begin{tabular}{|c|c|c|c|}
\hline \multirow[b]{2}{*}{ Radionuclide } & \multirow{2}{*}{$\begin{array}{c}\text { Effective Dose } \\
\text { Coefficient (Sv/Bq) }\end{array}$} & \multicolumn{2}{|c|}{ Inhalation Dose Factor } \\
\hline & & Sv/d per $\mathrm{Bq} / \mathrm{m}^{3 \mathrm{~b}}$ & Sv/d per $\mathrm{Bq} / \mathrm{m}^{3 \mathrm{c}}$ \\
\hline${ }^{90} \mathrm{Sr}$ & $1.62 E-07$ & 1.60E-06 & 5.93E-06 \\
\hline${ }^{99} \mathrm{Tc}$ & 1.30E-08 & $1.29 \mathrm{E}-07$ & 4.77E-07 \\
\hline${ }^{126} \mathrm{Sn}$ & 2.85E-08 & 2.82E-07 & $1.04 \mathrm{E}-06$ \\
\hline${ }^{137} \mathrm{Cs}$ & $3.90 \mathrm{E}-08$ & 3.87E-07 & 1.43E-06 \\
\hline${ }^{210} \mathrm{~Pb}$ & 9.99E-06 & 9.91E-05 & 3.67E-04 \\
\hline${ }^{226} \mathrm{Ra}$ & 9.53E-06 & $9.45 E-05$ & $3.50 \mathrm{E}-04$ \\
\hline${ }^{227} \mathrm{AC}$ & $5.69 E-04$ & $5.64 \mathrm{E}-03$ & $2.09 E-02$ \\
\hline${ }^{229} \mathrm{Th}$ & $2.56 \mathrm{E}-04$ & 2.54E-03 & $9.40 \mathrm{E}-03$ \\
\hline${ }^{230} \mathrm{Th}$ & $1.00 \mathrm{E}-04$ & $9.92 E-04$ & 3.67E-03 \\
\hline${ }^{232} \mathrm{Th}+{ }^{228} \mathrm{Ra}+{ }^{228} \mathrm{Th}$ & 1.70E-04 & $1.68 \mathrm{E}-03$ & $6.23 E-03$ \\
\hline${ }^{231} \mathrm{~Pa}$ & $1.40 \mathrm{E}-04$ & 1.39E-03 & $5.14 \mathrm{E}-03$ \\
\hline${ }^{232} \mathrm{U}+{ }^{228} \mathrm{Th}$ & 8.06E-05 & $8.00 E-04$ & 2.96E-03 \\
\hline${ }^{233} \mathrm{U}$ & $9.60 \mathrm{E}-06$ & $9.52 E-05$ & $3.52 E-04$ \\
\hline${ }^{234} U$ & $9.40 \mathrm{E}-06$ & 9.32E-05 & 3.45E-04 \\
\hline${ }^{236} U$ & 8.70E-06 & 8.63E-05 & 3.19E-04 \\
\hline${ }^{238} U$ & 8.01E-06 & 7.94E-05 & 2.94E-04 \\
\hline${ }^{237} \mathrm{~Np}$ & 5.00E-05 & $4.96 E-04$ & $1.84 \mathrm{E}-03$ \\
\hline${ }^{238} \mathrm{Pu}$ & $1.10 \mathrm{E}-04$ & $1.09 E-03$ & $4.04 \mathrm{E}-03$ \\
\hline${ }^{239} \mathrm{Pu}$ & $1.20 E-04$ & 1.19E-03 & 4.40E-03 \\
\hline${ }^{240} \mathrm{Pu}$ & $1.20 \mathrm{E}-04$ & 1.19E-03 & 4.40E-03 \\
\hline${ }^{242} \mathrm{Pu}$ & 1.10E-04 & $1.09 \mathrm{E}-03$ & $4.04 \mathrm{E}-03$ \\
\hline${ }^{241} \mathrm{Am}$ & $9.60 \mathrm{E}-05$ & 9.52E-04 & $3.52 E-03$ \\
\hline${ }^{243} \mathrm{Am}$ & 9.60E-05 & $9.52 E-04$ & $3.52 \mathrm{E}-03$ \\
\hline
\end{tabular}

Source: The calculations are shown in Excel spreadsheet Dose Factor Calculations_Rev 4.xls (Appendix B).

Notes: $\quad{ }^{a}$ From Table 6.3-1.

${ }^{b}$ Calculated by multiplying the effective dose coefficient for inhalation by the term calculated in Table 6.3-3 $\left(9.92 \mathrm{~m}^{3} / \mathrm{d}\right)$.

${ }^{c}$ Can be obtained from the values in the preceding column by multiplying them by unit conversion of 3.7 (100 rem/Sv*0.037 Bq/pCi). 
INTENTIONALLY LEFT BLANK 


\section{CONCLUSIONS}

This section summarizes the derivation of volcanic ash exposure scenario BDCFs and recommendations for their use in the TSPA model, as well as the derivation of inhalation dose factors for the eruption phase of the volcanic event. The output of this analysis is included in several data sets, as shown in Tables 7-1 and 7.2 for the analysis using ICRP 30 and ICRP 72 dosimetric methods, respectively.

Table 7-1. Output Data Tracking Numbers - ICRP 30 Dosimetric Methods

\begin{tabular}{|l|l|l|}
\hline \multicolumn{1}{|c|}{ DTN } & \multicolumn{1}{|c|}{ DTN Title } & \multicolumn{1}{|c|}{ DTN Description and Comments } \\
\hline MO0407MWDBMVAE.000 & $\begin{array}{l}\text { Biosphere Dose Conversion Factors for the } \\
\text { Volcanic Ash Exposure Scenario }\end{array}$ & $\begin{array}{l}\text { BDCFs for the volcanic ash exposure } \\
\text { scenario calculated using ICRP 30- } \\
\text { based methods. }\end{array}$ \\
\hline MO0407SPADFIDV.000 & $\begin{array}{l}\text { Dose Factors for Calculating Inhalation } \\
\text { Dose During Volcanic Eruption }\end{array}$ & $\begin{array}{l}\text { Dose factors for calculating inhalation } \\
\text { dose during volcanic eruption calculated } \\
\text { using ICRP 30-based methods. }\end{array}$ \\
\hline MO0407MWDGSMFV.000 & $\begin{array}{l}\text { GoldSim Biosphere Model Files for } \\
\text { Calculating Biosphere Dose Conversion } \\
\text { Factors for Volcanic Ash Exposure } \\
\text { Scenario }\end{array}$ & $\begin{array}{l}\text { GoldSim files used for calculation of } \\
\text { BDCFs for the volcanic ash exposure } \\
\text { scenario using ICRP 30-based } \\
\text { dosimetric methods. }\end{array}$ \\
\hline
\end{tabular}

Table 7-2. Output Data Tracking Numbers - ICRP 72 Dosimetric Methods

\begin{tabular}{|l|l|l|}
\hline \multicolumn{1}{|c|}{ DTN } & \multicolumn{1}{|c|}{ DTN Title } & \multicolumn{1}{|c|}{ DTN Description and Comments } \\
\hline MO0501MWDVABDC.000 & $\begin{array}{l}\text { Biosphere Dose Conversion Factors for the } \\
\text { Volcanic. Ash Exposure Scenario } \\
\text { Calculated Using ICRP 72 Dose } \\
\text { Coefficients }\end{array}$ & $\begin{array}{l}\text { BDCFs for the volcanic ash exposure } \\
\text { scenario calculated using ICRP 72- } \\
\text { based methods. }\end{array}$ \\
\hline MO0501SPAVAINH.000 & $\begin{array}{l}\text { Dose Factors for Evaluating Inhalation } \\
\text { Dose During Volcanic Eruption, Calculated } \\
\text { Using ICRP 72 Dose Coefficients }\end{array}$ & $\begin{array}{l}\text { Dose factors for calculating inhalation } \\
\text { dose during volcanic eruption calculated } \\
\text { using ICRP 72-based methods. }\end{array}$ \\
\hline MO0503MWDGBMVA.000 & $\begin{array}{l}\text { GoldSim Biosphere Model Files Used for } \\
\text { Calculation of Volcanic Ash Scenario } \\
\text { BDCFs Based on ICRP 72 Dosimetric } \\
\text { Methods }\end{array}$ & $\begin{array}{l}\text { GoldSim files used for calculation of } \\
\text { BDCFs for the volcanic ash exposure } \\
\text { scenario using ICRP 72-based } \\
\text { dosimetric methods. }\end{array}$ \\
\hline
\end{tabular}

The values of the BDCFs were developed using the ERMYN biosphere model and remain valid within the application and validation limits of the model (BSC 2004 [DIRS 169460], Section 8.2). Specifically, the BDCFs were developed for a specific assessment context, described in the Biosphere Model Report (BSC 2004 [DIRS 169460]), the reference biosphere, and the receptor (the RMEI). If used for other situations, the BDCFs may not apply.

For the volcanic ash exposure scenario, the model applies to a layer of small ash particles that could be resuspended into the air. The model and the BDCFs do not apply to other volcanic media, such as contaminated gas, lava, or coarse ash. Some assumptions regarding the model development (BSC 2004 [DIRS 169460], Section 6.3.2.4) are based on thin ash deposits; if thick ash deposits occur, the model might overestimate the BDCF values. For example, the external exposure is assumed in the biosphere model to originate from a contaminated ground surface. If 
the radionuclides were distributed in a thick layer of ash/soil, the external exposure would be reduced because of self-absorption of the fraction of radiation emitted from contaminated ash/soil. The biosphere model assumes that the entire deposited activity is mixed into the surface soil and available to plants. For the activity distributed throughout a layer thicker than the rooting depth of plants, a portion of the deposited activity would not be available for plant uptake.

The ERMYN model does not apply during a volcanic eruption when volcanic ash is still in the air (i.e., before initial settling on the ground). For the eruption period, the inhalation dose factors should be used.

It is recommended that the values developed using the updated dosimetric methods (ICRP 72) be used in the TSPA model (Table 7-2). This includes DTN: MO0501MWDVABDC.000 containing BDCFs for the volcanic ash exposure scenario and DTN: MO0501SPAVAINH.000 containing inhalation dose factors for calculating inhalation dose during volcanic eruption.

The basis for recommending the use of ICRP 72-based BDCFs is to provide a more accurate estimate of annual dose using a more current scientific understanding. This recommendation is supported by conclusions reached by an International Atomic Energy Agency International Review Team (IRT), which reviewed the biosphere modeling done by the U.S. Department of Energy for the Yucca Mountain Site Characterization Project in 2001 (IAEA 2001 [DIRS 155188]). In their review the IRT stated that ICRP Publication 72 was the preferred basis for the international approach to dosimetric modeling and noted that "even if the [dosimetric] data are fixed in regulatory compliance calculations, it is important to test the implications of applying more modern dosimetric data such as presented in ICRP Publications 72 and 74. The IRT observes that ICRP 72 data are adopted in the IAEA Basic Safety Standards (IAEA 1996 [DIRS 156655]) and have legal force in EU [European Union] Member States through implementation of the EURATOM Directive 96/29" (IAEA 2001 [DIRS 155188], p. 43).

\subsection{ANALYSIS SUMMARY}

\subsubsection{Incorporation of Uncertainty}

The outputs of the biosphere model are BDCFs for volcanic ash exposure scenario. To incorporate uncertainty in the model input, BDCFs were calculated in a series of 1,000 model realizations. The resulting probability distribution represents uncertainty in the BDCFs. The full set of BDCFs consists of the three BDCF components generated for each of 23 primary radionuclides and each model realization. The summary of these results, in the form of discrete cumulative probability distributions of BDCFs in 5 percentile increments, plus means and standard deviations, is presented in Tables 6.2-1 to 6.2-3 for the external exposure-ingestionradon component, short-term inhalation component, and the long-term inhalation component, respectively. The full set of $\mathrm{BDCF}$ vectors for each realization is included on a CD-ROM (Appendix B) in the file $V A B D C F$ Realizations $M C \_$Rev 4.xls.

Consideration of uncertainty was not included in the calculation of the inhalation dose factors because these parameters were developed as a scoping tool for the TSPA, as explained in Section 6.3.2. 


\subsubsection{Format of the Biosphere Dose Conversion Factor Input to TSPA}

Three BDCF components are calculated for each primary radionuclide: $B D C F_{\text {ext,ing,Rn,i, }}$ $B D C F_{\text {inh, }, \text {, }}$, and $B D C F_{\text {inh }, p, i}$ (Section 6.2.2). The first component $\left(B D C F_{\text {ext,ing,Rn, },}\right)$ is time independent and accounts for three exposure pathways (ingestion, inhalation of radon decay products, and external exposure). The second and third BDCF components ( $B D C F_{\text {inh, }, i}$ and $\left.B D C F_{i n h, p, i}\right)$ account for inhaling airborne particulates. Both of these components depend on ash thickness. The second component $\left(B D C F_{\text {inh }, i, i}\right)$ represents short-term inhalation exposure during increased concentrations of airborne particulates following volcanic eruption and is timedependent because mass loading will gradually decrease after an eruption. The third component $\left(B D C F_{\text {inh, }, i, i}\right)$ represents long-term inhalation of suspended particulates under nominal conditions (i.e., when the mass loading is not elevated as the result of volcanic eruption). The results of the BDCF calculations are in the format of 1,000 row vectors, one for each model realization, consisting of three BDCF components for each of the 23 primary radionuclides (i.e., $69 \mathrm{BDCF}$ components per vector) and a value of critical thickness of ash corresponding to the specific $\mathrm{BDCF}$ vector. A vector can be regarded as a one-dimensional array containing the results of a single realization of the biosphere model for the primary radionuclides. Some BDCFs include contributions from their decay products. The primary radionuclides (radionuclides tracked in the TSPA) and the decay products, which were included in the BDCF with the primary radionuclides, are shown in Table 7.1-1.

Table 7.1-1. Primary Radionuclides and Their Decay Products Included in the BDCFs for the Volcanic Ash Exposure Scenario

\begin{tabular}{|c|l|}
\hline Primary Radionuclide & \multicolumn{1}{|c|}{ Short-lived Decay Products Included in BDCF } \\
\hline Sr-90 & Y-90 \\
\hline Tc-99 & \\
\hline Sn-126 & \\
\hline Cs-137 & Ba-137m \\
\hline Pb-210 & Bi-210, Po-210 \\
\hline Ra-226 & Rn-222, Po-218, Pb-214, At-218, Bi-214, Po-214, Tl-210 \\
\hline Ac-227 & Th-227, Fr-223, Ra-223, Rn-219, Po-215, Pb-211, Bi-211, Tl-207, Po-211 \\
\hline Th-229 & Ra-225, Ac-225, Fr-221, At-223, Bi-213, Po-213, Tl-209, Pb-209 \\
\hline Th-230 & \\
\hline Th-232 & Ra-228, Ac-228, Th-228, Ra-224, Rn-220, Po-216, Pb-212, Bi-212, Po-212, Tl-208 \\
\hline Pa-231 & \\
\hline$U-232$ & Th-228, Ra-224, Rn-220, Po-216, Pb-212, Bi-212, Po-212, Tl-208 \\
\hline U-233 & \\
\hline U-234 & \\
\hline U-236 & \\
\hline U-238 & Th-234, Pa-234m, Pa-234 \\
\hline Np-237 & Pa-233 \\
\hline Pu-238 & \\
\hline Pu-239 & \\
\hline Pu-240 & \\
\hline Pu-242 & \\
\hline Am-241 & \\
\hline Am-243 & Np-239 \\
\hline
\end{tabular}




\subsubsection{Use of Biosphere Dose Conversion Factors for Volcanic Ash Exposure Scenario}

Calculating the annual all-pathway dose for any given primary radionuclide is done by combining the source term with the BDCFs. In the case of the volcanic ash exposure scenario, the mass-loading decrease function and the ash thickness also are factored into the calculations. The total annual dose is the sum of the annual doses from individual radionuclides tracked in the TSPA (primary radionuclides), including their decay products. The total annual dose, computed by the TSPA model, is calculated as

$$
\begin{aligned}
D_{\text {total }}\left(d_{a}, t\right)= & \sum_{i} B D C F_{i}\left(d_{a} t\right) \times C s_{i}(t) \\
= & \sum_{i} B D C F_{e x t, i n g, R n, i} \times C s_{i}(t) \\
& +\sum_{i}\left(B D C F_{i n h, v, i} f(t)+B D C F_{i n h, p, i}\right) g\left(d_{a}\right) \times C s_{i}(t)
\end{aligned}
$$

where

$$
\begin{aligned}
& D_{\text {total }}\left(d_{a}, t\right) \quad=\text { time- and ash thickness-dependent total annual dose to a defined } \\
& \text { receptor resulting from the volcanic release of radionuclides from the } \\
& \text { repository }(\mathrm{Sv} / \mathrm{yr}) \\
& C s_{i}(t) \quad=\text { time dependent activity concentration of radionuclide } i \text { in volcanic ash } \\
& \text { deposited on the ground }\left(\mathrm{Bq} / \mathrm{m}^{2}\right) \\
& B D C F_{i}\left(d_{a}, t\right)=\text { BDCF for primary radionuclide } i \text { for an ash depth } d_{a} \text { at time } t \\
& \text { following a volcanic eruption ( } \mathrm{Sv} / \mathrm{yr} \text { per } \mathrm{Bq} / \mathrm{m}^{2} \text { ) } \\
& B D C F_{\text {ext,ing,Rn,i}}=\mathrm{BDCF} \text { for primary radionuclide } i \text { for external exposure, ingestion, and } \\
& \text { inhalation of radon decay products ( } \mathrm{Sv} / \mathrm{yr} \text { per } \mathrm{Bq} / \mathrm{m}^{2} \text { ) } \\
& B D C F_{i n h, v, i}=\text { BDCF for primary radionuclide } i \text { for short-term inhalation at } \\
& \text { post-volcanic level of mass loading in excess of nominal mass loading } \\
& \left(\mathrm{Sv} / \mathrm{yr} \text { per } \mathrm{Bq} / \mathrm{m}^{2}\right) \\
& B D C F_{i n h, p, i}=\mathrm{BDCF} \text { for primary radionuclide } i \text { for long-term inhalation at nominal } \\
& \text { level of mass loading ( } \mathrm{Sv} / \mathrm{yr} \text { per } \mathrm{Bq} / \mathrm{m}^{2} \text { ). }
\end{aligned}
$$

The annual dose should be calculated using Equation 7.1-1 and BDCFs provided in DTN: MO0501MWDVABDC.000. The BDCF values in DTN: MO0501MWDVABDC.000 are given with three significant digits although only two significant digits are significant. This is to avoid round-off errors in the TSPA calculation of the annual dose.

The time function, $f(t)$, appearing in Equation 7:1-1 accounts for the reduction of mass loading in the years immediately following volcanic eruption. The mass loading is assumed to decrease exponentially with time as

$$
f(t)=e^{-\lambda t}
$$


where

$\lambda \quad=$ mass loading decrease constant $(1 / \mathrm{yr})$

$t \quad=\quad$ time (years); $t=0$ is the first year after a volcanic eruption.

The mass loading decrease constant (Equation 7.1-2) depends on the ash thickness, and for an initial ash depth of less than $10 \mathrm{~mm}$, it is represented by a triangular probability distribution function with a mode of $0.33 / \mathrm{yr}$, a minimum of $0.2 / \mathrm{yr}$, and a maximum of $2.0 / \mathrm{yr}$ (DTN: MO0407SPAINEXI.002 [DIRS 170597]). For an initial ash depth of $10 \mathrm{~mm}$ or more, the mass loading decrease constant is represented by a triangular distribution with a mode of $0.20 / \mathrm{yr}$, a minimum of $0.125 / \mathrm{yr}$, and a maximum of 1.0/yr (DTN: MO0407SPAINEXI.002 [DIRS 170597]).

The function of ash thickness, $g\left(d_{a}\right)$, is expressed as

$$
g\left(d_{a}\right)= \begin{cases}1 & \text { when } d_{a}<d_{c} \\ \frac{d_{c}}{d_{a}} & \text { when } d_{a} \geq d_{c}\end{cases}
$$

where

$$
d_{c} \quad=\quad \text { critical thickness of ash layer }(\mathrm{mm})
$$

The value of the critical thickness is different for each biosphere model realization and has to be sampled in the TSPA code together with the BDCFs from the same vector.

Equations shown above use SI units for consistency with the documentation of the ERMYN model (BSC 2004 [DIRS 169460]). However, any units can be used to define parameters in GoldSim, where the ERMYN model is implemented, as long as they are dimensionally consistent. The values of BDCF components in the output DTN are given in $\mathrm{rem} / \mathrm{yr}$ per $\mathrm{pCi} / \mathrm{m}^{2}$.

\subsubsection{Use of Inhalation Dose Factors}

For the eruption period, the dose factors for the inhalation exposure pathway should be used instead of the BDCFs. The inhalation dose factors for evaluating doses during volcanic eruptions are listed in Tables 6.3-4 and 6.3-5 for the ICRP 30- and ICRP 72-based dosimetric methods, respectively. To calculate the daily dose from inhaling a specific radionuclide during a volcanic eruption, multiply the activity concentration of that radionuclide in air by the appropriate inhalation dose factors. The total inhalation dose, $D_{i n h}$, from concentrations of primary radionuclides in air is then calculated as

$$
D_{i n h}=\sum_{i} D_{i n h, i}=\sum_{i} D F_{i} \times C a_{i, o u t d o o r}
$$

where 


$$
\begin{aligned}
& D_{i n h, i}=\text { daily inhalation dose for a primary radionuclide } i(\mathrm{~Sv} / \mathrm{d} \text { or rem } / \mathrm{d}) \\
& D F_{i} \quad=\text { Inhalation dose factor for a primary radionuclide } i\left(\mathrm{~Sv} / \mathrm{d} \mathrm{per} \mathrm{Bq} / \mathrm{m}^{3}\right. \text { or } \\
& \mathrm{rem} / \mathrm{d} \text { per } \mathrm{pCi} / \mathrm{m}^{3} \text { ) } \\
& \mathrm{Ca}_{i, \text { outdoor }}=\text { activity concentration of a primary radionuclide } i \text { in outdoor air for the } \\
& \text { ash that has not yet fallen on the ground }\left(\mathrm{Bq} / \mathrm{m}^{3} \text { or } \mathrm{pCi} / \mathrm{m}^{3}\right) \text {. }
\end{aligned}
$$

\subsubsection{Correlations, Pathway Analysis, and Climate Change}

Correlation coefficients for the BDCF components for each individual radionuclide are listed in Tables 6.2-7 and 6.2-8 for the ICRP 30- and ICRP 72-based calculations, respectively. Correlations between the BDCF components of different radionuclides also have been calculated. Correlation coefficients for the external-ingestion-radon component are listed in Tables 6.2-10 and 6.2-11 for the ICRP 30- and ICRP 72-based calculations, respectively. Correlation coefficients are the highest for the isotopes of actinides. Correlation coefficients for short-term and long-term inhalation components are equal to one.

Results of pathway analysis are presented in Tables 6.2-12 and 6.2-13 for the ICRP 30- and ICRP 72-based calculations, respectively . The dominant pathway for actinide radionuclides is inhalation, with the long-term component contributing about $2 / 3$ of the dose and the short-term component contributing about $1 / 3$ of the dose. The pathway contributions for the other radionuclides are more diversified. For example, the food consumption pathways are dominant for ${ }^{99} \mathrm{Tc}$, and the external exposure pathway is dominant for ${ }^{137} \mathrm{Cs}$.

The climate change during the period of time considered in this analysis has negligible effects on the BDCFs for the volcanic ash exposure scenario because it primarily affects parameters related to irrigation and water use, which are not included in the model for the volcanic ash exposure scenario. It is thus recommended that the present-day climate BDCFs be used for dose assessment for the entire period.

\subsection{HOW ACCEPTANCE CRITERIA ARE ADDRESSED}

The primary function of this analysis was to calculate BDCFs for the volcanic ash exposure scenario. As noted before, this analysis thus integrates the model (BSC 2004 [169460]) and the model input parameters (BSC 2005 [DIRS 172827]; BSC 2004 [DIRS 169672]; BSC 2004 [DIRS 169673]; BSC 2004 [DIRS 169458]; and BSC 2004 [DIRS 169459]) to produce the output of the biosphere model. All acceptance criteria addressed by the model and input parameters reports are implicitly included in this analysis and the modeling results. The results reflect consideration of the site-specific FEPs, characteristics of the reference biosphere and its features, parameter selection and justification, as well as incorporation of uncertainty in the model and its input parameters. The model for the volcanic ash exposure scenario is briefly described in Section 6.1.5; the model input parameter values, including uncertainty distributions, are presented in Section 4.1.

In addition, the acceptance criteria, identified as applicable to this analysis in Section 4.2 , that are related to the model abstraction were addressed. The biosphere modeling does not utilize the model abstraction step, but rather, the BDCF are calculated as the model output. In this sense, the BDCFs, which are the input to the TSPA model, serve as a "collapsed" model abstraction. 
The uncertainty in the model and its input parameters is propagated onto and reflected in the BDCFs through the development of the BDCF vectors representing individual model realizations (see Section 7.1.2), consistent with the acceptance criteria 2.2.1.3.13.3, 2.2.1.3.13.4, and 2.2.1.3.14.3 (see Table 4.2-2).

The specific acceptance criteria were addressed as follows.

\subsection{3: Redistribution of Radionuclides in Soils}

Acceptance Criterion 1 (1): As discussed in Sections 1 and 7.1.4, because the BDCFs are used directly in the TSPA, the TSPA adequately incorporates the redistribution of radionuclides contained in soils as a result of volcanic activity.

Acceptance Criterion 1(2): By including the BDCFs for disruptive events, the TSPA identifies and describes an aspect of radionuclide redistribution in soil that is important to repository performance. See, Section 7.1.2 and 7.1.3. The technical bases for the volcanic ash exposure scenario are adequately described in Section 6.1.2.

Acceptance Criterion 1 (3): Relevant site FEPs, including climate change (Section 6.1.3), receptor characteristics (Section 6.1.4), and the biosphere model (Section 6.1.5) have been appropriately modeled in the BDCFs. Sufficient technical bases are provided for the BDCFs.

Acceptance Criterion 2 (1): The BDCF development process described in Section 6 shows that the BDCFs used in the license application are justified. Adequate descriptions of how data was used to develop BDCFs are presented in Section 6.2, which includes pathway analysis (Section 6.2.4), climate change (Section 6.2.5), and uncertainty (Section 6.2.1).

Acceptance Criterion 2 (2): As shown in Section 4.1, sufficient data are available to adequately define the parameters for the BDCFs.

Acceptance Criterion 3 (1): The calculations described in Section 6.2.2, the calculation results presented in Section 6.2.3, and the development of inhalation dose factors described in Section 6.3.2 show that the parameter values are technically defensible, reasonably account for uncertainties, and do not result in an under-representation of the risk.

Acceptance Criterion 3 (2): Section 6.3.1 shows that the data on the airborne particulate concentration are based on the resuspension of appropriate material in a climate and level of disturbance which is expected to be found at the location of the RMEI in the event of a volcanic eruption.

Acceptance Criterion 3 (3): Sections 6.2 .1 and 7.1.1 show that uncertainty was adequately | represented in the BDCFs by a probability distribution which was generated from a thousand realizations using a Monte Carlo technique.

\subsection{3: Biosphere Characteristics}

Acceptance Criterion 1 (3): Section 6.2.5 shows how the impact of climate change on the BDCFs | is consistent with assumptions about climate change in other TSPA abstractions. 
Acceptance Criterion 2 (1): The BDCF development process described in Section 6 shows that the BDCFs used in the license application are justified. Adequate descriptions of how data was used to develop BDCFs are presented in Section 6.2, which includes a pathway analysis (Section 6.2.4), climate change (Section 6.2.5), and uncertainty (Section 6.2.1).

Acceptance Criterion 2 (2): As shown in Section 4.1, sufficient data are available to adequately | define the parameters for the BDCFs.

Acceptance Criterion 3 (1): Section 4.1 shows that the parameter values are technically defensible, reasonably account for uncertainties, do not result in an under-representation of the risk, and are consistent with the definition of the RMEI.

Acceptance Criterion 3 (2): Section 4.1 shows that the technical bases for the parameter values and ranges used to generate the BDCFs are consistent with site characterization data and technically defensible. 


\section{INPUTS AND REFERENCES}

\subsection{DOCUMENTS CITED}

Baxter, P.J.; Bonadonna, C.; Dupree, R.; Hards, V.L.; Kohn, S.C.; Murphy, M.D.;

Nichols, A.; Nicholson, R.A.; Norton, G.; Searl, A.; Sparks, R.S.J.; and Vickers, B.P. 1999. "Cristobalite in Volcanic Ash of the Soufriere Hills Volcano, Montserrat, British West Indies." Science, 283, 1142-1145. Washington, D.C.: American Association for the Advancement of Science. TIC: 248128.

BSC (Bechtel SAIC Company) 2003. Total System Performance Assessment-License Application Methods and Approach. TDR-WIS-PA-000006 REV 00 ICN 01. Las Vegas, Nevada: Bechtel SAIC Company. ACC: DOC.20031215.0001.

BSC 2004. Agricultural and Environmental Input Parameters for the Biosphere Model. ANL-MGR-MD-000006 REV 02. Las Vegas, Nevada: Bechtel SAIC Company. ACC: DOC.20040915.0007.

BSC 2004. Atmospheric Dispersal and Deposition of Tephra from a Potential 166296 Volcanic Eruption at Yucca Mountain, Nevada. MDL-MGR-GS-000002 REV 01. Las Vegas, Nevada: Bechtel SAIC Company. ACC: DOC.20041025.0003.

BSC 2004. Biosphere Model Report. MDL-MGR-MD-000001 REV 01. Las Vegas, Nevada: Bechtel SAIC Company. ACC: DOC.20041108.0005.

BSC 2004. Disruptive Event Biosphere Dose Conversion Factor Analysis. ANLMGR-MD-000003 REV 03. Las Vegas, Nevada: Bechtel SAIC Company. ACC: DOC.20040908.0007.

BSC 2004. Environmental Transport Input Parameters for the Biosphere Model. ANL-MGR-MD-000007 REV 02. Las Vegas, Nevada: Bechtel SAIC Company. ACC: DOC.20040913.0003.

BSC 2004. Future Climate Analysis. ANL-NBS-GS-000008 REV 01. Las Vegas, Nevada: Bechtel SAIC Company. ACC: DOC.20040908.0005.

BSC 2004. Inhalation Exposure Input Parameters for the Biosphere Model. ANLMGR-MD-000001 REV 03. Las Vegas, Nevada: Bechtel SAIC Company. ACC: DOC.20040913.0001.

BSC 2004. Soil-Related Input Parameters for the Biosphere Model. ANL-NBS-MD000009 REV 02. Las Vegas, Nevada: Bechtel SAIC Company.

ACC: DOC.20040913.0002. 
BSC 2005. Characteristics of the Receptor for the Biosphere Model. ANL-MGRMD-000005 REV 04. Las Vegas, Nevada: Bechtel SAIC Company.

ACC: DOC.20050405.0005.

BSC 2005. Q-List. 000-30R-MGR0-00500-000-001. Las Vegas, Nevada: Bechtel SAIC Company. ACC: ENG.20050217.0010.

BSC 2005. Technical Work Plan for Biosphere Modeling and Expert Support. TWPNBS-MD-000004 REV 04. Las Vegas, Nevada: Bechtel SAIC Company. ACC: DOC.20050131.0012.

BSC 2005. Technical Work Plan for: TSPA-LA FY 05-06 Activities. TWP-MGR-PA000031 REV 00. Las Vegas, Nevada: Bechtel SAIC Company. ACC:

DOC.20050401.0006.

Buist, A.S.; Martin, T.R.; Shore, J.H.; Butler, J.; and Lybarger, J.A. 1986. "The Development of a Multidisciplinary Plan for Evaluation of the Long-Term Health Effects of the Mount St. Helens Eruptions." Chapter 4 of Health Effects of Volcanoes: An Approach to Evaluating the Health Effects of an Environmental Hazard. Buist, A.S. and Bernstein, R.S., eds. American Journal of Public Health, Volume 76, Supplement. Washington, D.C.: American Public Health Association. TIC: 246677.

Canori, G.F. and Leitner, M.M. 2003. Project Requirements Document. TER-MGRMD-000001 REV 02. Las Vegas, Nevada: Bechtel SAIC Company.

166275 ACC: DOC.20031222.0006.

Craighead, J.E.; Adler, K.B.; Butler, G.B.; Emerson, R.J.; Mossman, B.T.; and Woodworth, C.D. 1983. "Biology of Disease, Health Effects of Mount St. Helens Volcanic Dust." Laboratory Investigation, 48, (1), 5-12. Baltimore, Maryland: Williams \& Wilkins. TIC: 250154.

CRWMS (Civilian Radioactive Waste Management System) M\&O (Managing and Operating Contractor) 2000. Total System Performance Assessment for the Site Recommendation. TDR-WIS-PA-000001 REV 00 ICN 01. Las Vegas, Nevada: CRWMS M\&O. ACC: MOL.20001220.0045.

Eckerman, K.F. and Ryman, J.C. 1993. External Exposure to Radionuclides in Air, Water, and Soil, Exposure-to-Dose Coefficients for General Application, Based on the 1987 Federal Radiation Protection Guidance. EPA 402-R-93-081. Federal Guidance Report No. 12. Washington, D.C.: U.S. Environmental Protection Agency, Office of Radiation and Indoor Air. TIC: 225472. 
Eckerman, K.F.; Wolbarst, A.B.; and Richardson, A.C.B. 1988. Limiting Values of Radionuclide Intake and Air Concentration and Dose Conversion Factors for Inhalation, Submersion, and Ingestion. EPA 520/1-88-020. Federal Guidance Report No. 11. Washington, D.C.: U.S. Environmental Protection Agency. ACC: MOL.20010726.0072.

GoldSim Technology Group. 2003. User's Guide, GoldSim Probabilistic Simulation Environment. Version 8.01. Redmond, Washington: Golder Associates. TIC: 255170.

Gordian, M.E.; Ozkaynak, H.; Xue, J.; Morris, S.S.; and Spengler, J.D. 1996.

"Particulate Air Pollution and Respiratory Disease in Anchorage, Alaska."

Environmental Health Perspectives, 104, (3), 290-297. Research Park Triangle, North Carolina: National Institute of Environmental Health Sciences, National Institutes of Health. TIC: 250157.

IAEA (International Atomic Energy Agency) 1996. International Basic Safety Standards for Protection Against Ionizing Radiation and for the Safety of Radiation Sources. Safety Series No. 115. Vienna, Austria: International Atomic Energy Agency. TIC: 250383.

IAEA 2001. An International Peer Review of the Biosphere Modelling Programme of the US Department of Energy's Yucca Mountain Site Characterization Project, Report of the IAEA International Review Team. Vienna, Austria: International Atomic Energy Agency. TIC: 250092.

ICRP (International Commission on Radiological Protection) 1977. Recommendations of the International Commission on Radiological Protection. Volume 1, No. 3 of Annals of the ICRP. ICRP Publication 26. Reprinted 1982. New York, New York: Pergamon Press. TIC: 221568

ICRP 1979. Limits for Intakes of Radionuclides by Workers. Volume 2, No. 3/4 of Annals of the ICRP. Sowby, F.D., ed. ICRP Publication 30 Part 1. New York, New York: Pergamon Press. TIC: 4939.

ICRP 1980. Limits for Intakes of Radionuclides by Workers. Volume 4, No. 3/4 of Annals of the ICRP. Sowby, F.D., ed. ICRP Publication 30 Part 2. Reprinted 1990. Elmsford, New York: Pergamon Press. TIC: 4941.

ICRP 1981. Limits for Intakes of Radionuclides by Workers. Volume 6, No. 2/3 of Annals of the ICRP. Sowby, F.D., ed. ICRP Publication 30 Part 3, Including Addendum to Parts 1 and 2. New York, New York: Pergamon Press. TIC: 4943. 
ICRP 1991. "1990 Recommendations of the International Commission on Radiological Protection." Volume 21, No. 1-3 of Annals of the ICRP. ICRP

Publication 60. New York, New York: Pergamon Press. TIC: 235864.

ICRP'1996. Age-Dependent Doses to Members of the Public from Intake of Radionuclides: Part 5 Compilation of Ingestion and Inhalation Dose Coefficients. Volume 26, No. 1 of Annals of the ICRP. Smith, H., ed. ICRP Publication 72. New York, New York: Pergamon Press. TIC: 235870.

Johnson, K.G.; Loftsgaarden, D.O.; and Gideon, R.A. 1982. "The Effects of Mount St. Helens Volcanic Ash on the Pulmonary Function of 120 Elementary School Children." American Review of Respiratory Disease, 126, (6), 1066-1069.

New York, New York: American Lung Association. TIC: 250159.

Lide, D.R. and Frederikse, H.P.R., eds. 1997. CRC Handbook of Chemistry and Physics. 78th Edition. Boca Raton, Florida: CRC Press. TIC: 243741.

McGimsey, R.G.; Neal, C.A.; and Riley, C.M. 2001. Areal Distribution, Thickness, Mass, Volume, and Grain Size of Tephra-Fall Deposits from the 1992 Eruptions of Crater Peak Vent, Mt. Spurr Volcano, Alaska. Open-File Report 01-370. Anchorage, Alaska: U.S. Geological Survey. TIC: 253361.

Merchant, J.A.; Baxter, P.; Bernstein, R.; McCawley, M.; Falk, H.; Stein, G.; Ing, R.; 160102 and Attfield, M. 1982. "Health Implications of the Mount St. Helens' Eruption: Epidemiological Considerations." Annals of Occupational Hygiene, 26, (1-4), 911 919. New York, New York: Pergamon Press. TIC: 253237.

Nania, J.M.; Garcia, M.R.; Fruchter, J.S.; Olsen, K.B.; and Hooper, P.R. 1994. "In the Shadow of El Chichon: An Overview of the Medical Impact of the 28 March to 4 April 1982 Eruptions of the Mexican Volcano." Prehospital and Disaster Medicine, 9, (1), 77/58 through 85/66. Solano Beach, California: Jems Publishing. TIC: 250160 .

NRC (U.S. Nuclear Regulatory Commission) 2003. Yucca Mountain Review Plan, Final Report. NUREG-1804, Rev. 2. Washington, D.C.: U.S. Nuclear Regulatory Commission, Office of Nuclear Material Safety and Safeguards. TIC: 254568.

Sarna-Wojcicki, A.M.; Shipley, S.; Waitt, R.B., Jr.; Dzurisin, D.; and Wood, S.H. 160227 1982. "Areal Distribution, Thickness, Mass, Volume, and Grain Size of Air-Fall Ash from the Six Major Eruptions of 1980." The 1980 Eruptions of Mount St. Helens, Washington. Professional Paper 1250. 577-600. Reston, Virginia: U.S. Department of the Interior, U.S. Geological Survey. TIC: 218260. 
Searl, A.; Nicholl, A.; and Baxter, P.J. 2002. "Assessment of the Exposure of Islanders to Ash from the Soufriere Hills Volcano, Montserrat, British West Indies." Occupational and Environmental Medicine, 59, (8), 523-531. Clifton, New Jersey: BMJ Publishing Group. TIC: 253212.

Sjoreen, A.L.; Ramsdell, J.V., Jr.; McKenna, T.J.; McGuire, S.A.; Fosmire, C.; and 164093 Athey, G.F. 2001. RASCAL 3.0: Description of Models and Methods. NUREG-1741. Washington, D.C.: U.S. Nuclear Regulatory Commission. TIC: 254458.

Steel, R.G.D. and Torrie, J.H. 1980. Principles and Procedures of Statistics: $A$ 150857 Biometrical Approach. 2nd Ed. New York, New York: McGraw Hill. TIC: 6322.

Yano, E.; Yokoyama, Y.; Higashi, H.; Nishii, S.; Maeda, K.; and Koizumi, A. 1990. 160112 "Health Effects of Volcanic Ash: A Repeat Study." Archives of Environmental Health, 45, (6), 367-373. Washington, D.C.: Heldref Publications. TIC: 250162.

\subsection{CODES, STANDARDS, REGULATIONS, AND PROCEDURES}

10 CFR 63. 2004 Energy: Disposal of High-Level Radioactive Wastes in a Geologic Repository at Yucca Mountain, Nevada. ACC: MOL.20050323.0071.

AP-2.22Q, Rev. 1, ICN 1. Classification Analyses and Maintenance of the Q-List. Washington, D.C.: U.S. Department of Energy, Office of Civilian Radioactive Waste Management. ACC: DOC.20040714.0002.

LP-2.29Q-BSC, Rev. 0, ICN 0. Planning for Science Activities. Washington, D.C.: U.S. Department of Energy, Office of Civilian Radioactive Waste Management. ACC: DOC.20050114.0001.

LP-SIII.9Q-BSC, Rev. 0, ICN 0. Scientific Analyses. Washington, D.C.: U.S. Department of Energy, Office of Civilian Radioactive Waste Management. ACC: DOC.20050203.0010.

LP-SI.11Q-BSC Rev. 0, ICN 1. Software Management. Washington, D.C.: U.S. Department of Energy, Office of Civilian Radioactive Waste Management. ACC: DOC.20041005.0008.

\subsection{SOURCE DATA, LISTED BY DATA TRACKING NUMBER}

MO0008SPATSP00.013. Total Suspended Particle Concentrations - Washington 151750 1979-1982. Submittal date: 08/02/2000.

MO0306MWDBGSMF.001. Biosphere Goldsim Model Files. Submittal date: 06/13/2003. 
MO0403SPAAEIBM.002. Agricultural and Environmental Input Parameters for the 169392 Biosphere Model. Submittal date: 03/22/2004.

MO0406SPAETPBM.002. Environmental Transport Input Parameters for the Biosphere Model. Submittal date: 06/24/2004.

MO0407SPACRBSM.002. Characteristics of the Receptor for the Biosphere Model. Submittal date: 07/19/2004.

MO0407SEPFEPLA.000. LA FEP List. Submittal date: 07/20/2004.

MO0407SPAINEXI.002. Inhalation Exposure Input Parameters for the Biosphere Model. Submittal date: 07/12/2004.

MO0407SPASRPBM.002. Soil Related Parameters for the Biosphere Model.

Submittal date: 07/20/2004.

MO0501SEPFEPLA.001. LA FEP List and Screening. Submittal date: 01/17/2005.

MO0503SPADCESR.000. Dose Coefficients for Internal and External Exposure to Selected Radionuclides Consistent with ICRP 72 Dosimetric Methods. Submittal date: 03/01/2005.

\subsection{SOFTWARE CODES}

BSC 2003. Software Code: GoldSim. V7.50.100. PC. 10344-7.50.100-00.

BSC 2004. Software Code: GoldSim. V8.01 Service Pack 4. PC, Windows 2000. 10344-8.01SP4-00.

BSC 2004. Software Code: GoldSim. V.8.02.300. PC, Windows 2000. 10344-8.02-03.

\subsection{OUTPUT DATA, LISTED BY DATA TRACKING NUMBER}

MO0407MWDBMVAE.000 Biosphere Dose Conversion Factors for the Volcanic Ash Exposure Scenario

MO0407SPADFIDV.000

Dose Factors for Calculating Inhalation Dose During Volcanic Eruption

MO0407MWDGSMFV.000

GoldSim Biosphere Model Files for Calculating Biosphere Dose Conversion Factors for Volcanic Ash Exposure Scenario 
MO0501MWDVABDC.000 Biosphere Dose Conversion Factors for the Volcanic Ash Exposure Scenario Calculated Using ICRP 72 Dose Coefficients

MO0501SPAVAINH.000 Dose Factors for Evaluating Inhalation Dose During Volcanic Eruption, Calculated Using ICRP 72 Dose Coefficients

MO0503MWDGBMVA.000 GoldSim Biosphere Model Files Used for Calculation of Volcanic Ash Scenario BDCFs Based on ICRP 72 Dosimetric Methods 
INTENTIONALLY LEFT BLANK 


\section{APPENDIX A}

LIST AND DESCRIPTION OF GOLDSIM FILES GENERATED IN THIS ANALYSIS 


\section{A. LIST AND DESCRIPTION OF GOLDSIM FILES GENERATED IN THIS ANALYSIS}

This appendix contains the list and description of GoldSim biosphere model files generated in this and in the previous revision of the analysis. The files are included in DTN: MO0503MWDGBMVA.000 and MO0407MWDGSMFV.000, for the ICRP 72- and ICRP 30based BDCFs, respectively.

Figure A-1 shows the list of GoldSim files for the present-day climate (file names ERMYN_VA_M_<radionuclide symbol>ICRP72) generated using GoldSim 8.02 .300 with ICRP 72-based dosimetric inputs. These files are included in DTN: MO0503MWDGBMVA.000.

\begin{tabular}{|c|c|c|c|c|}
\hline \multirow[b]{4}{*}{ Modern Climate } & Name 1 . & Size & Type & Modfied \\
\hline & DERMYN_VA_M_AC227D_ICRP72.gsm & $8,135 \mathrm{~KB}$ & GoldSim Model & 1/13/2005 9:56 AM \\
\hline & DERMYN_VA_M_Am241_ICRP72.gsm & $8,135 \mathrm{~KB}$ & GoldSim Model & 1/13/2005 11:39 AM \\
\hline & DERMYN_VA_M_Am2430_ICRP72.gsm & $8,135 \mathrm{~KB}$ & GoldSim Model & 1/13/2005 11:41 AM \\
\hline \multirow{3}{*}{ It This folder is Online. } & DERMYN_VA_M_CS137D_ICRP72.gsm & $8,135 \mathrm{~KB}$ & GoldSim Model & 1/13/2005 9:21 AM \\
\hline & DERMYN_VA_M_Np2370_ICRP72.gsm & $8,135 \mathrm{~KB}$ & GoldSim Model & 1/13/2005 11:27 AM \\
\hline & DERMYN_VA_M_Pa231_ICRP72.gsm & $8,135 \mathrm{~KB}$ & Goldsim Model & $1 / 13 / 200510: 56 \mathrm{AM}$ \\
\hline Select an item to view its description. & DERMYN_VA_M_Pb2100_ICRP72.gsm & $8,135 \mathrm{~KB}$ & GoldSim Model & 1/13/2005 9:23 AM \\
\hline See also: & פERMYN_VA_M_Pu238_ICRP72.gsm & $8,135 \mathrm{kB}$ & GoldSim Model & 1/13/2005 11:29 AM \\
\hline My Documents & DERMYN_VA_M_Pu239_ICRP72.gsm & $8,135 \mathrm{~KB}$ & GoldSim Model & 1/13/2005 11:31 AM \\
\hline My Network Places & DERMYN_YA_M_PU240_ICRP72.gsm & $8,135 \mathrm{~KB}$ & GoldSim Model & 1/13/2005 11:35 AM \\
\hline \multirow{15}{*}{ My Computer } & DERMVN_VA_M_PU242_ICRP72.gsm & $8,135 \mathrm{kB}$ & Goldsim Model & 1/13/2005 11:37 AM \\
\hline & DERMYN_VA_M_Ra2260_ICRP72.gsm & $8,135 \mathrm{~KB}$ & GoldSim Model & 1/13/2005 9:27 AM \\
\hline & DERMYN_VA_M_Ra228D_ICRP72.gsm & $8,135 \mathrm{~KB}$ & GoldSim Model & 1/13/2005 9:53 AM \\
\hline & DERMYN_YA_M_Sn126D_ICRP72.gsm & $8,135 \mathrm{kB}$ & GoldSim Model & 1/13/2005 9:19 AM \\
\hline & DERMYN_VA_M_Sr900_ICRP72.gsm & $8,135 \mathrm{~KB}$ & GoldSim Model & 1/13/2005 9:13 AM \\
\hline & DERMYN_VA_M_TC99_ICRP72.gsm & $8,135 \mathrm{~KB}$ & GoldSim Model & 1/13/2005 9:16 AM \\
\hline & DERMYN_YA_M_Th228D_ICRP72.gsm & $8,135 \mathrm{~KB}$ & GoldSim Model & 1/13/2005 9:58 AM \\
\hline & DERMYN_YA_M_Th2290_ICRP72.gsm & $8,135 \mathrm{~KB}$ & GoldSim Model & 1/13/2005 9:59 AM \\
\hline & DERMYN_YA_M_Th230_ICRP72.gsm & $8,135 \mathrm{kB}$ & GoldSim Model & 1/13/2005 10:52 AM \\
\hline & DERMYN_VA_M_Th232_ICRP72.gsm & $8,135 \mathrm{~KB}$ & GoldSim Model & 1/13/2005 10:54 AM \\
\hline & DERMYN_VA_M_U232_ICRP72.gsm & 8, $135 \mathrm{~KB}$ & GoldSim Model & 1/13/2005 11:08 AM \\
\hline & DERMYN_VA_M_U233_ICRP72.gsm & $8,135 \mathrm{~KB}$ & GoldSim Model & 1/13/2005 11:09 AM \\
\hline & DERMYN_VA_M_U1234_ICRP72.gsm & $8,135 \mathrm{~KB}$ & Goldsim Model & 1/13/2005 11:11 AM \\
\hline & DERMYN_YA_M_U236_ICRP72.gsm & $8,135 \mathrm{~KB}$ & GoldSim Model & 1/13/2005 11:13 AM \\
\hline & DERMYN_YA_M_U238D_ICRP72.gsm & $8,135 \mathrm{~KB}$ & GoldSim Model & 1/13/2005 11:15 AM \\
\hline & & & $198 \mathrm{MB}$ & ⿹ㅡㅇ Local intranet \\
\hline
\end{tabular}

Figure A-1. GoldSim Files for Calculating Volcanic Scenario BDCFs for the Present-Day Climate Generated Using ICRP 72-Based Dosimetric Inputs 
Figure A-2 shows the list of GoldSim files for the upper bound of the glacial transition climate (file names ERMYN_VA_F_<radionuclide symbol> ICRP72) generated using GoldSim 8.02.300 with ICRP 72-based dosimetric inputs. These files are included in DTN: MO0503MWDGBMVA.000.

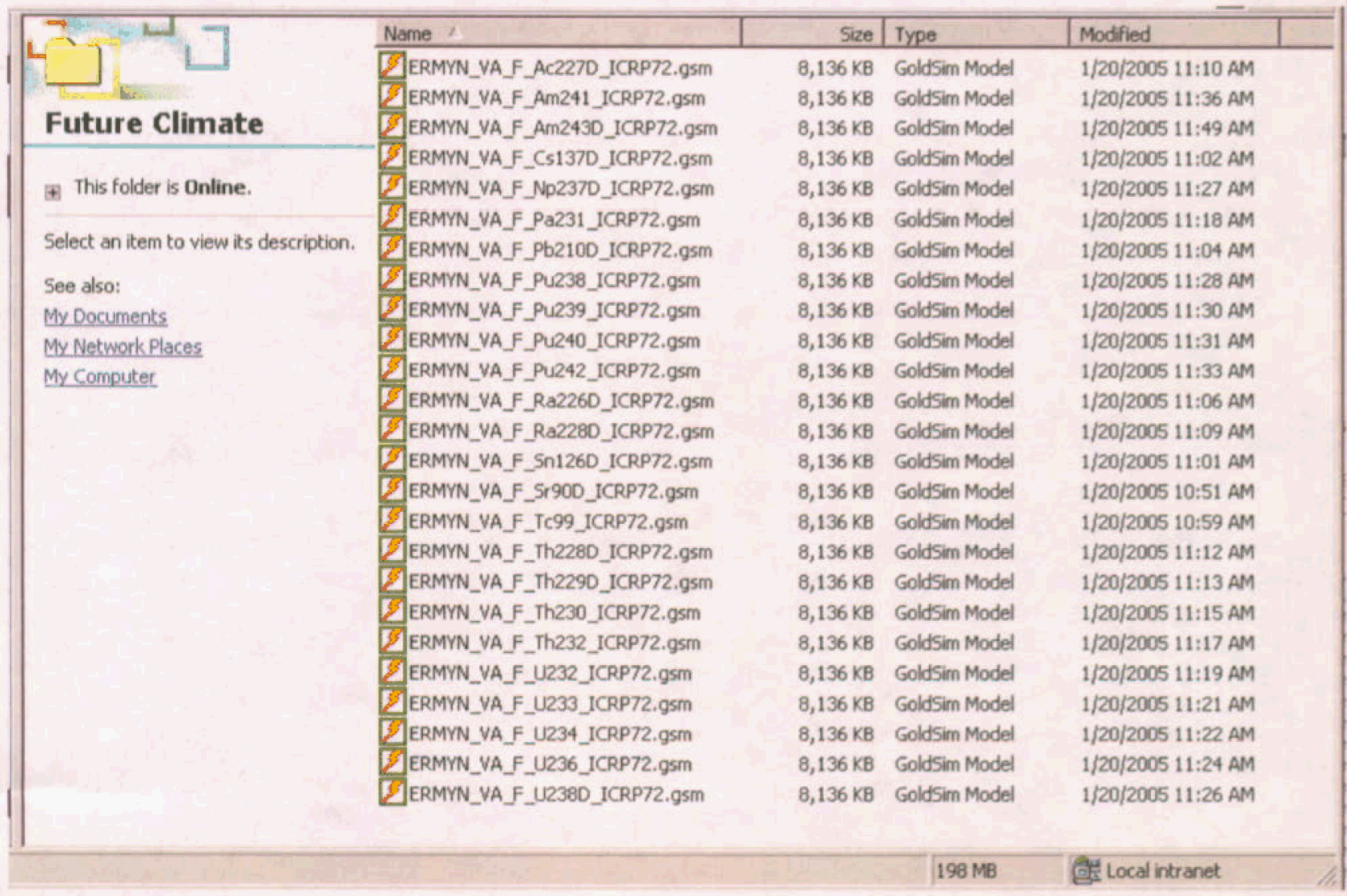

Figure A-2. GoldSim Files for Calculating Volcanic Scenario BDCFs for the Upper Bound of the Glacial Transition Climate Generated Using ICRP 72-Based Dosimetric Inputs 
Figure A-3 shows the list of GoldSim files for the present-day climate (file names ERMYN_VA_MC8_<radionuclide symbol $>$ ) generated using GoldSim 8.01 Service Pack 4 with dosimetric inputs based on ICRP 30 methods. These files are included in DTN: MO0407MWDGSMFV.000.

\begin{tabular}{|c|c|c|c|c|}
\hline \multirow{5}{*}{$\begin{array}{l}\text { 801SP4 Runs for } \\
\text { Modern Climate }\end{array}$} & Name $A$ & Size & Type & Modified \\
\hline & DERMYN_VA_MC8_Ac2270.gsm & $8,126 \mathrm{~KB}$ & GoldSim Model & $6 / 30 / 200412: 16 \mathrm{PM}$ \\
\hline & EERMYN_YA_MC8_Am241.gsm & $8,126 \mathrm{~KB}$ & GoldSim Model & $7 / 1 / 20047: 38 \mathrm{AM}$ \\
\hline & [DERMYN_VA_MC8_Am243D.gsm & $8,126 \mathrm{~KB}$ & GoldSim Model & $7 / 1 / 20047: 43$ AM \\
\hline & BERMYN_VA_MC8_Cs137D.gsm & $8,126 \mathrm{~KB}$ & GoldSim Model & $6 / 30 / 200411: 21$ AM \\
\hline \multirow{21}{*}{$\begin{array}{l}\text { This folder is Online. } \\
\text { Select an item to view its description. } \\
\text { See also: } \\
\text { My Documents } \\
\text { My Network Places } \\
\text { My Computer }\end{array}$} & BERMYN_VA_MC8_Np237D.gsm & $8,126 \mathrm{~KB}$ & GoldSim Model & $7 / 1 / 20047: 25$ AM \\
\hline & 2ERMYN_VA_MC8_Pa231.gsm & $8,126 \mathrm{~KB}$ & GoldSim Model & $6 / 30 / 20044: 15 \mathrm{PM}$ \\
\hline & E]ERMYN_VA_MC8_Pb210D.gsm & $8,126 \mathrm{~KB}$ & GoldSim Model & $6 / 30 / 200411: 24$ AM \\
\hline & BERMYN_VA_MC8_Pu238.gsm & $8,126 \mathrm{~KB}$ & GoldSim Model & $7 / 1 / 20047: 28$ AM \\
\hline & DERMYN_VA_MC8_Pu239.gsm & $8,126 \mathrm{~KB}$ & GoldSim Model & $7 / 1 / 20047: 31$ AM \\
\hline & DERMYN_VA_MC8_PU240.gsm & $8,126 \mathrm{~KB}$ & GoldSim Model & 7/1/2004 7:34 AM \\
\hline & DERMYN_VA_MC8_PU242.gsm & $8,126 \mathrm{kB}$ & GoldSim Model & $7 / 1 / 20047: 36$ AM \\
\hline & DERMYN_VA_MC8_Ra226D.gsm & $8,126 \mathrm{~KB}$ & GoldSim Model & $6 / 30 / 200411: 28 \mathrm{AM}$ \\
\hline & DERMYN_VA_MC8_Ra228D.gsm & $8,126 \mathrm{~KB}$ & GoldSim Model & 6/30/2004 11:30 AM \\
\hline & DERMVN_VA_MC8_Sn1260.gsm & $8,126 \mathrm{~KB}$ & GoldSim Model & $6 / 30 / 200411: 18$ AM \\
\hline & DERMYN_VA_MC8_5r900.gsm & $8,126 \mathrm{~KB}$ & GoldSim Model & $6 / 30 / 200410: 59$ AM \\
\hline & BERMYN_VA_MC8_Tc99.gsm & $8,126 \mathrm{~KB}$ & GoldSim Model & 6/30/2004 11:10 AM \\
\hline & [DERMYN_VA_MC8_Th2280.gsm & $8,126 \mathrm{~KB}$ & GoldSim Model & 6/30/2004 12:20 PM \\
\hline & DERMYN_YA_MC8_Th2290.gsm & $8,126 \mathrm{~KB}$ & GoldSim Model & $6 / 30 / 200412: 23 \mathrm{PM}$ \\
\hline & DERMVN_VA_MC8_Th230.gsm & $8,126 \mathrm{~KB}$ & GoldSim Model & $6 / 30 / 200412: 28 \mathrm{PM}$ \\
\hline & DERMYN_VA_MC8_Th232.gsm & $8,126 \mathrm{~KB}$ & GoldSim Model & $6 / 30 / 20044: 11$ PM \\
\hline & DERMYN_VA_MC8_U232.gsm & $8,126 \mathrm{~KB}$ & GoldSim Model & $6 / 30 / 20044: 20 \mathrm{PM}$ \\
\hline & (2)ERMYN_VA_MC8_U233.gsm & $8,126 \mathrm{~KB}$ & GoldSim Model & $6 / 30 / 20044: 23 \mathrm{PM}$ \\
\hline & 2]ERMYN_VA_MC8_U234.gsm & $8,126 \mathrm{~KB}$ & GoldSim Model & 6/30/2004 4:26 PM \\
\hline & DERMYN_VA_MC8_U236.gsm & $8,126 \mathrm{~KB}$ & GoldSim Model & $6 / 30 / 20044: 48 \mathrm{PM}$ \\
\hline & [DERMYN_VA_MC8_U238D.gsm & $8,126 \mathrm{~KB}$ & GoldSim Model & 7/1/2004 7:22 AM \\
\hline
\end{tabular}

Figure A-3. GoldSim Files for Calculating Volcanic Scenario BDCFs for the Present-Day Climate Using GoldSim 8.01 Service Pack 4 and ICRP 30-Based Dosimetric Inputs 
Figure A-4 shows the list of GoldSim files for the upper bound of the glacial transition climate (file names ERMYN_VA_FC8_<radionuclide symbol $>$ ) generated using GoldSim 8.01 Service Pack 4 with dosimetric inputs based on ICRP 30 methods. These files are included in DTN: MO0407MWDGSMFV.000.

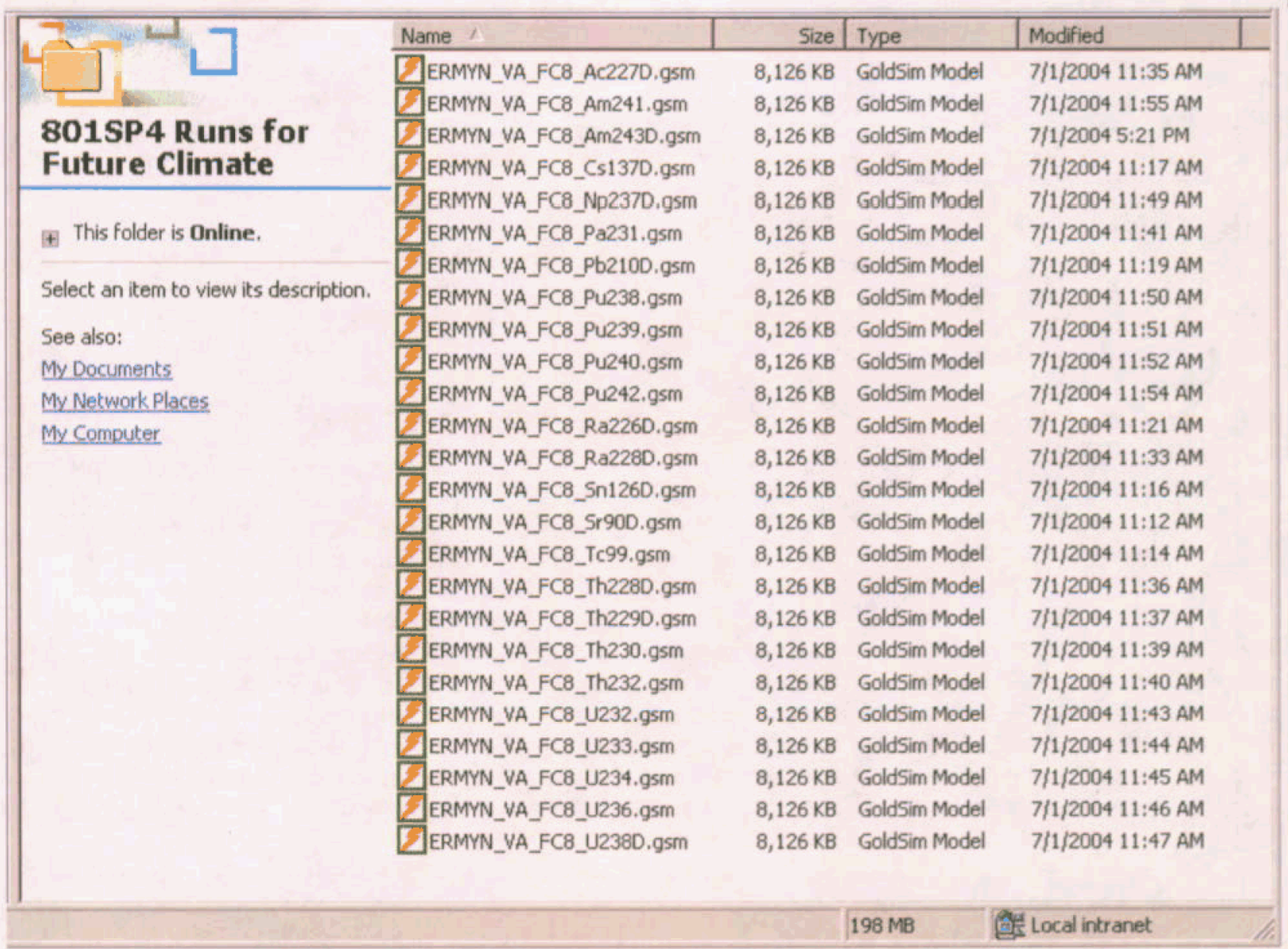

Figure A-4. GoldSim Files for Calculating Volcanic Scenario BDCFs for the Upper Bound of the Glacial Transition Climate Using GoldSim 8.01 Service Pack 4 and ICRP 30-Based Dosimetric Inputs 


\section{APPENDIX B}

\section{DESCRIPTION OF EXCEL FILES GENERATED IN THIS ANALYSIS}




\section{B. DESCRIPTION OF EXCEL FILES GENERATED IN THIS ANALYSIS}

This appendix contains descriptions of the Microsoft Excel files that were used for calculations in this analysis. For the files that contain the stochastic results of the GoldSim runs, the values were copied from the RESULTS container of the appropriate GoldSim files and pasted into the worksheets. The files can be found on the CD ROM included as an electronic attachment to this document.

\section{B.1. LIST OF EXCEL FILES}

Figure B-1 shows the list of the Excel files supporting this analysis. The description of the files follows in Section B.2.

\begin{tabular}{|c|c|c|c|c|}
\hline - & Name $A$ & Size & Type & Modified \\
\hline & Dose Factor Calculations_Rev 3 , xls & $18 \mathrm{~KB}$ & Microsoft Excel Worksheet & $3 / 10 / 20053: 59$ PM \\
\hline & $\$$ Dose Factor Calculations_Rev 4 , xls & $27 \mathrm{~KB}$ & Microsoft Excel Worksheet & $3 / 29 / 2005$ 12:16 PM \\
\hline & 12) VA BDCF FC Pathway Analysis_Rev 3.x/s & $7,524 \mathrm{~KB}$ & Microsoft Excel Worksheet & 3/10/2005 3:49 PM \\
\hline & 1]VA BDCF FC Pathway Analysis_Rev 4,xls & $7,524 \mathrm{~KB}$ & Microsoft Excel Worksheet & $3 / 10 / 20053: 49 \mathrm{PM}$ \\
\hline & 2] VA BDCF MC Correlations_Rev 4, xls & $2,865 \mathrm{~KB}$ & Microsoft Excel Worksheet & $3 / 29 / 200511: 23$ AM \\
\hline & X]VA BDCF MC Pathway Analysis_Rev 3.xis & $7,531 \mathrm{~KB}$ & Microsoft Excel Worksheet & $3 / 29 / 200511: 36$ AM \\
\hline & 13VA BDCF MC Pathway Analysis_Rev 4,x/s & $7,541 \mathrm{~KB}$ & Microsoft Excel Worksheet & $4 / 28 / 200511: 07$ AM \\
\hline \multirow{2}{*}{\multicolumn{2}{|c|}{-1}} & $4,288 \mathrm{~KB}$ & Microsoft Excel Worksheet & $3 / 29 / 200511: 02$ AM \\
\hline & & & $36.4 \mathrm{MB}$ & Local intranet \\
\hline
\end{tabular}

Figure B-1. List of Excel Files Supporting This Analysis

\section{B.2. DESCRIPTION OF EXCEL FILES}

VA BDCF Realizations MC_Rev 4.xls-This Excel file contains the results of 1,000 biosphere model realizations that generated BDCFs for the volcanic ash exposure scenario for the presentday climate. The file contains BDCFs calculated using ICRP 30-based dosimetric methods as well as BDCFs calculated using ICRP 72-based methods. The workbook consists of three worksheets: BDCF Components ICRP72, BDCF Components ICRP30, and Comparison. The first two worksheets contain BDCF components calculated using ICRP 72 and ICRP 30-based methods. BDCF components are arranged by radionuclide in sets of four columns per radionuclide in Columns B to DE. For each realization and each radionuclide, three BDCF components were generated: external-ingestion-radon, short-term inhalation, and long-term inhalation. The fourth value per radionuclide is the critical thickness, which is the same for a given model realization for each radionuclide. The results of individual realizations are in rows 36 to 1,035 . The values were copied from the BDCF All Dist, BDCF InhShort Dist, $B D C F$ InhLong Dist and Crit Thickness elements from the Results container for the GoldSim files for individual radionuclides.

Row 7 contains the means of the values in rows 36 to 1,035 of the corresponding columns, calculated using the Excel AVERAGE function for the specified cell range. 
Row 8 contains standard deviations of the values in rows 36 to 1,035 of the corresponding columns, calculated using the Excel STDEV function for the specified cell range.

Row 10 contains minima of the values in rows 36 to 1,035 of the corresponding columns, calculated using the Excel MIN function for the specified cell range.

Row 30 contains maxima of the values in rows 36 to 1,035 of the corresponding columns, calculated using the Excel MAX function for the specified cell range.

Rows 11 to 29 contain the percentiles in the increments of 5 of the values in rows 36 to 1,035 of the corresponding columns, calculated using the Excel PERCENTILE function for the specified cell range.

The worksheet Comparison contains comparison of the mean values of the BDCF components calculated using ICRP 30 and ICRP 72 dosimetric methods.

Row 10 contains the mean value of the BDCFs for external-ingestion-radon, short-term inhalation, and long-term inhalation for each radionuclide using ICRP 72 based dose coefficients.

Row 14 contains the mean value of the BDCFs for external-ingestion-radon, short-term inhalation, and long-term inhalation for each radionuclide using ICRP 30 based dose coefficients.

Row 18 calculates the ratio of BDCFs for ICRP 72 methodology to ICRP 30 methodology for external-ingestion-radon, short-term inhalation, and long-term inhalation for each radionuclide.

Row 22 calculates the percent difference in the BDCFs for ICRP 72 methodology relative to the BDCFs using ICRP 30 methodology.

Also, included in this worksheet are graphs showing percent difference between the BDCF components are generated. They appear in the report in Section 6.2.3.

VA BDCF MC Correlations_Rev 4.xls-This Excel file contains calculations of the correlation coefficients for the BDCF components. The file contains correlation coefficients for BDCFs calculated using ICRP 30-based dosimetric methods as well as for BDCFs calculated using ICRP 72-based methods. The workbook consists of two worksheets: ICRP 30-based values, and ICRP 72-based values.

In these two worksheets the BDCF components were copied from the file VA BDCF Realizations MC_Rev 4.xls and are arranged by radionuclide in sets of 3 columns per radionuclide in Columns $B$ to $B R$. For each realization and each radionuclide, the three BDCF components (external-ingestion-radon, short-term inhalation, and long-term inhalation) are in the first, second, and third column of each set, respectively. The BDCF component values for individual model realizations are in rows 12 to 1,011 .

Row 11 contains the correlation coefficients for the BDCF components for a given radionuclide. The correlation coefficients were calculated using the Excel CORREL function. 
Rows 1,017 to 1,039 contain the 23 by 23 table of correlation coefficients for the | external-ingestion-radon BDCF components for all 23 primary radionuclides.

The rows beneath contain supplementary calculations of the Student's $t$ values for the range of correlation coefficient values and the summary of the BDCF component correlation coefficients shown in row 11 of the worksheet.

\section{VA BDCF MC Pathway Analysis_Rev 3.xls and VA BDCF MC Pathway} Analysis_Rev 4.xls - These Excel file contain calculations of pathway contributions to BDCFs for the present-day climate for the BDCFs calculated using ICRP 30- and ICRP 72-based methods, respectively. Each workbook consists of 28 worksheets, containing pathway BDCFs for individual realizations and individual radionuclides. The first worksheet (Pathway Summary) contains the summary of the mean pathway BDCFs. The second to twenty-eighth worksheets contain the pathway BDCFs from individual realizations for 23 primary radionuclides, 2 longlived decay products, ${ }^{228} \mathrm{Ra}$ and ${ }^{228} \mathrm{Th}$, and the 2 decay products combined with their respective primary radionuclide, i.e., ${ }^{232} \mathrm{Th}+{ }^{228} \mathrm{Ra}+{ }^{228} \mathrm{Th}$ and ${ }^{232} \mathrm{U}+{ }^{228} \mathrm{Th}$. The mean values are also included. The pathway BDCFs for 1,000 realizations are in rows 10 to 1,009 of each spreadsheet for an individual radionuclide. The values were copied from GoldSim element BDCF_Pathway_Dist from the Results container following each run of the model. Row 6 contains the mean values calculated using the Excel AVERAGE function.

The Pathway Summary worksheet contains the mean values of pathway BDCFs copied from the individual radionuclide worksheets. These values are shown in rows 9 to 35 for individual radionuclides and in Columns $\mathrm{C}$ to $\mathrm{O}$ for individual exposure pathways. Column $\mathrm{P}$ contains the sum of the external, ingestion and radon pathways. Column $Q$ contains the total BDCF for $a$ radionuclide, which is a sum of individual pathway BDCFs.

Rows 43 to 69 contain the calculated percent values of the individual pathway contributions to the total BDCF. These values were calculated by dividing the mean pathway BDCFs by the total BDCF for a given radionuclide. Rows 72 to 94 contain the percent pathway contributions for pasting into the report. This worksheet also generates the plots shown in Section 6.2.5.

Column $\mathrm{U}$ to column $\mathrm{AH}$, rows 10 through 18, implements the methodology in Equation 6.2-7 to calculate the BDCF over time following a volcanic eruption. The Excel function EXP calculates " $\mathrm{e}$ " raised to the power of a number, where " $\mathrm{e}$ " is the base of the natural logarithm.

Column $\mathrm{U}$ to column $\mathrm{AH}$, rows 49 through 60 , implement the methodology in equations $6.2-8$ and 6.2-12.

VA BDCF FC Pathway Analysis_Rev 3.xls and VA BDCF FC Pathway Analysis_Rev 4.xls -These Excel files contain calculations of pathway contributions to BDCFs for the future climate represented by the upper bound of the glacial transition climate. The workbook consists of 28 worksheets, containing pathway BDCFs for individual realizations and individual radionuclides. The first worksheet (Pathway Summary) contains a summary of the mean pathway BDCFs and a comparison with the present-day climate pathway BDCFs from files VA BDCF MC Pathway Analysis_Rev 3.xls and VA BDCF MC Pathway Analysis_Rev 4.xls for the BDCFs calculated using ICRP 30- and ICRP 72-based methods, respectively. The layout of the workbook for the 
future climate is similar to the workbook for the present-day climate. The values were copied from the GoldSim pathway results summary, following each run of the model. Row 6 contains the mean values calculated using the Excel AVERAGE function.

The Pathway Summary worksheet contains the mean values of pathway BDCFs copied from the individual radionuclide worksheets. These values are shown in rows 9 to 35 for individual radionuclides and in Columns $\mathrm{C}$ to $\mathrm{O}$ for individual exposure pathways. Column $\mathrm{P}$ contains the sum of the external, ingestion and radon pathways. Column Q contains the total BDCF for a radionuclide, which is a sum of individual pathway BDCFs.

Rows 76 to 102 contain the summary of the present-day climate pathway BDCF results copied from the VA BDCF MC Pathway Analysis_Rev 3.xls file (Pathway Summary worksheet, rows 9 to 35) and VA BDCF MC Pathway Analysis_Rev 4.xls for the BDCFs calculated using ICRP 30 and ICRP 72-based methods, respectively. Rows 109 to 131 contain the ratios of pathway BDCFs for the future and the present-day climates.

Dose Factor Calculations_Rev 3.xls and Dose Factor Calculations_Rev 4.xls -These Excel files contain calculations of the inhalation dose factors computed using Equation 6.3-3 for the inhalation dose factors calculated using ICRP 30- and ICRP 72-based methods, respectively. The Excel file contains two worksheets; Calcs of Dose Factors and Dose Coefficients.

The worksheet Dose Coefficients calculates the effective dose coefficients for inhalation provided Tables 6.3.1 and 6.3.2 for ICRP 30 and ICRP 72 methodology respectively. The effective dose coefficients calculated in this worksheet are used in the worksheet Calcs of Dose Factors.

The worksheet Calcs of Dose Factors provide calculations of the effective breathing rate (term in the brackets in Equation 6.3-3) are in rows 10 to 19. Inhalation dose factors for individual radionuclides are calculated in rows 27 to 53 . Column $\mathrm{C}$ contains the values of effective dose coefficient for inhalation of a given radionuclide and its short-lived decay products, if applicable. Column $\mathrm{D}$ contains the value of the effective daily breathing rate calculated above. Columns $\mathrm{E}$ and $\mathrm{F}$ contain calculations of daily inhalation dose in Sv/d if the activity concentration in air is 1 $\mathrm{Bq} / \mathrm{m}^{3}$ and in rem/d for activity concentration of $1 \mathrm{pCi} / \mathrm{m}^{3}$, respectively. Daily doses are calculated as the product of daily activity intakes of a given radionuclide and the corresponding inhalation effective dose coefficient. 


\section{APPENDIX C}

VERIFICATION OF ERMYN MODEL FILES FOR GOLDSIM VERSIONS

8.01 SERVICE PACK 4 AND 8.02.300 


\section{VERIFICATION OF ERMYN MODEL FILES FOR GOLDSIM VERSIONS 8.01 SERVICE PACK 4 AND 8.02.300}

The ERMYN model for the volcanic ash exposure scenario (DTN: MO0306MWDBGSMF.001 [DIRS 163816]) was constructed and verified for GoldSim V7.50.100 (STN: 10344-7.50-10000) (BSC 2003 [161572]). This analysis was conducted using GoldSim V.8.02.300 (STN: 10344-8.02-03, Windows 2000) (BSC 2004 [DIRS 172588]) qualified under the Office of Civilian Radioactive Waste Management, Quality Assurance program for use on the Yucca Mountain Project. It also includes the previously developed BDCF, which were calculated using GoldSim V.8.01 Service Pack 4 (STN: 10344-8.01SP4-00, Windows 2000)(BSC 2004 [169695]).

Verification was conducted for two radionuclides: ${ }^{226} \mathrm{Ra}$, and ${ }^{239} \mathrm{Pu}$ because these two radionuclides are representative of all environmental transport and exposure pathways included in the model for the volcanic ash exposure scenario. To verify the applicability of GoldSim versions 8.01 Service Pack 4 and 8.02 .300 as software supporting the biosphere model, the original model verification files were opened in GoldSim versions 8.01 Service Pack 4 and 8.02.300 and the verification calculations were repeated.

Figure $\mathrm{C}-1$ shows the list of the original verification files as well as the model files generated in this analysis when the original files were run using GoldSim versions 8.01 Service Pack 4 and GoldSim 8.02.300. The model verification files are included in DTN: MO0503MWDGBMVA.000.

\begin{tabular}{|l|l|l|l|l|}
\hline & Name & Size & Type & Modfied \\
\hline & & $2,705 \mathrm{~KB}$ & GoldSim Model & $6 / 23 / 2003$ \\
\hline
\end{tabular}

Figure C-1. Model Verification Files Obtained Using GoldSim Versions 7.50.100, 8.01 Service Pack 4, and 8.02 .300

Table C-1 shows the results from the original verification files produced using GoldSim V.7.50.100 (BSC 2003 [161572]) and their re-runs in GoldSim V.8.01 Service Pack 4 (BSC 2004 [169695]) and GoldSim V.8.02.300 (BSC 2004 [DIRS 172588]). The results are identical, so the model can be executed using GoldSim V.8.01 Service Pack 4 as well as GoldSim V.8.02.300. 
Disruptive Event Biosphere Dose Conversion Factor Analysis

Table C-1. Results of Verification Runs Using GoldSim Versions 7.50.100, 8.01 Service Pack 4, and 8.02.300

\begin{tabular}{|c|c|c|c|}
\hline \multirow[b]{2}{*}{ Radionuclide } & \multirow[b]{2}{*}{ Pathway } & \multicolumn{2}{|c|}{ BDCF (rem/yr per pCi/L) } \\
\hline & & GoldSim V7.50.100 & GoldSim V8.01 Service Pack 4 and 8.02.300 \\
\hline \multirow[t]{14}{*}{ Pu-239 } & External & 1.392100E-11 & 1.392100E-11 \\
\hline & Inhalation Short & $6.899400 \mathrm{E}-07$ & 6.899400E-07 \\
\hline & Inhalation Long & $1.304500 \mathrm{E}-06$ & 1.304500E-06 \\
\hline & Radon & $0.000000 E+00$ & $0.000000 \mathrm{E}+00$ \\
\hline & Leafy vegetables & $1.683300 \mathrm{E}-11$ & $1.683300 \mathrm{E}-11$ \\
\hline & Other vegetables & $3.668600 \mathrm{E}-12$ & $3.668600 \mathrm{E}-12$ \\
\hline & Fruit & $1.558700 \mathrm{E}-11$ & $1.558700 \mathrm{E}-11$ \\
\hline & Grain & $1.222700 \mathrm{E}-12$ & $1.222700 \mathrm{E}-12$ \\
\hline & Meat & 2.678200E-13 & 2.678200E-13 \\
\hline & Milk & 1.045500E-14 & $1.045500 \mathrm{E}-14$ \\
\hline & Poultry & $9.577700 \mathrm{E}-14$ & $9.577700 \mathrm{E}-14$ \\
\hline & Eggs & 1.712200E-12 & 1.712200E-12 \\
\hline & Soil Ingestion & $3.445200 \mathrm{E}-10$ & $3.445200 \mathrm{E}-10$ \\
\hline & File name & ERMYN_VA_Pu239verf.gsm & $\begin{array}{l}\text { ERMYN_VA_Pu239verf_GS801SP4.gsm } \\
\text { ERMYN_VA_Pu239verf_GS802300.gsm }\end{array}$ \\
\hline \multirow[t]{14}{*}{ Ra-226 } & External & 7.848800 E-08 & 7.848800 E-08 \\
\hline & Inhalation Short & $1.382200 \mathrm{E}-08$ & $1.382200 \mathrm{E}-08$ \\
\hline & Inhalation Long & $2.613400 \mathrm{E}-08$ & $2.613400 \mathrm{E}-08$ \\
\hline & Radon & 8.033000 E- 08 & 8.033000E-08 \\
\hline & Leafy vegetables & $6.963500 \mathrm{E}-11$ & $6.963500 \mathrm{E}-11$ \\
\hline & Other vegetables & $2.171200 \mathrm{E}-11$ & $2.171200 \mathrm{E}-11$ \\
\hline & Fruit & $4.413500 \mathrm{E}-11$ & 4.413500E-11 \\
\hline & Grain & $2.720000 \mathrm{E}-12$ & $2.720000 \mathrm{E}-12$ \\
\hline & Meat & 1.330500E-11 & $1.330500 \mathrm{E}-11$ \\
\hline & Milk & $2.035000 \mathrm{E}-11$ & $2.035000 \mathrm{E}-11$ \\
\hline & Poultry & $5.267100 \mathrm{E}-13$ & $5.267100 \mathrm{E}-13$ \\
\hline & Eggs & $1.524800 \mathrm{E}-13$ & $1.524800 \mathrm{E}-13$ \\
\hline & Soil Ingestion & $1.291000 \mathrm{E}-10$ & 1.291000E-10 \\
\hline & File name & ERMYN_VA_Ra226verf.gsm & $\begin{array}{l}\text { ERMYN_VA_Ra226verf_GS801SP4.gsm } \\
\text { ERMYN_VA_Ra226verf_GS802300.gsm }\end{array}$ \\
\hline
\end{tabular}

Note: Files without a GoldSim version number ("_GS801SP4) in their title apply to V7.50.100. 


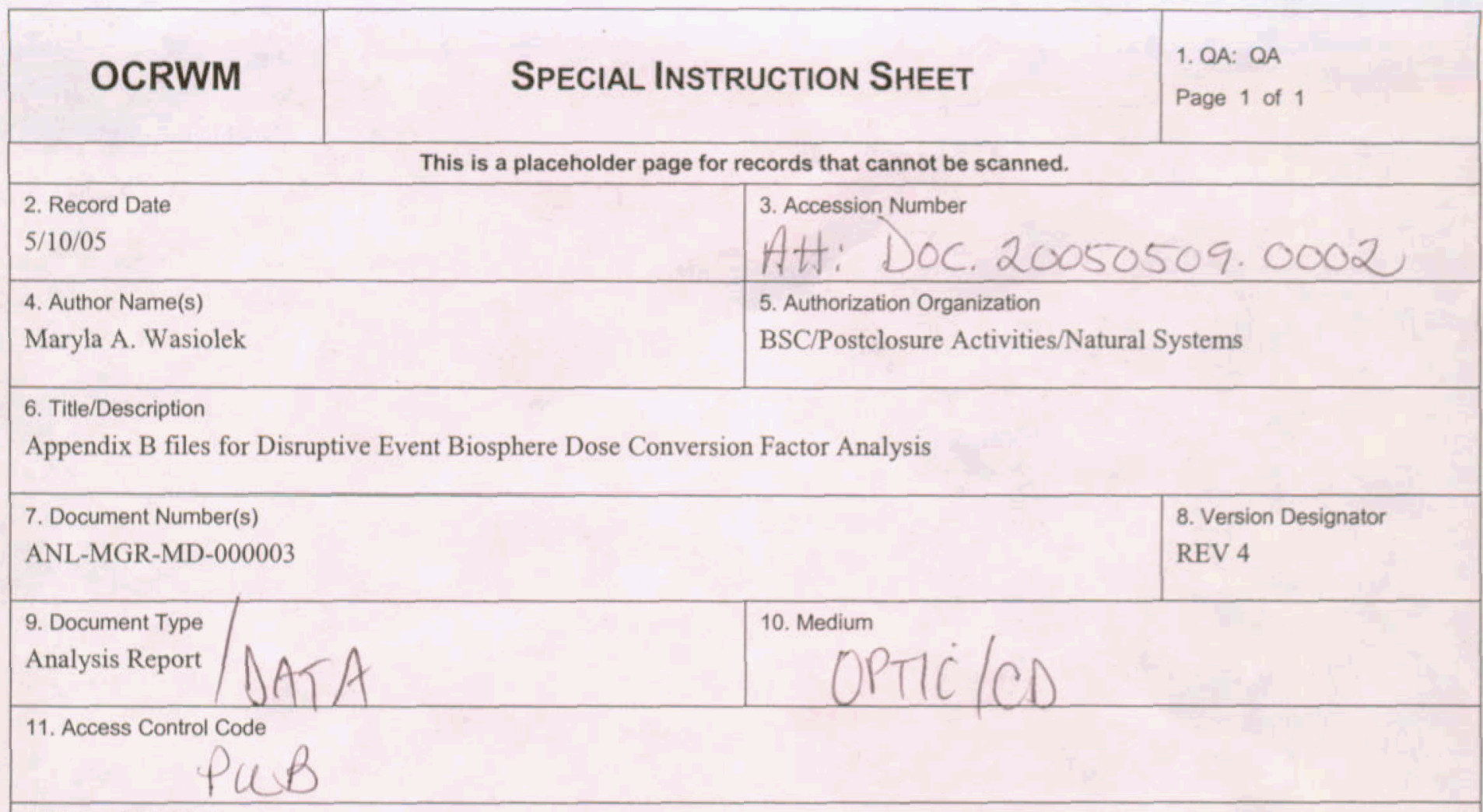

12. Traceability Designator

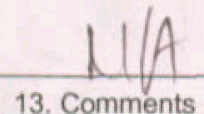

This CD contains Excel files accompanying the analysis report titled Disruptive Event Biosphere Dose Conversion Factor Analysis, ANL-MGR-MD-000003 REV 04. The files were created using Microsoft Excel 2000. These files are included in Appendix B of the document. The files are listed below.

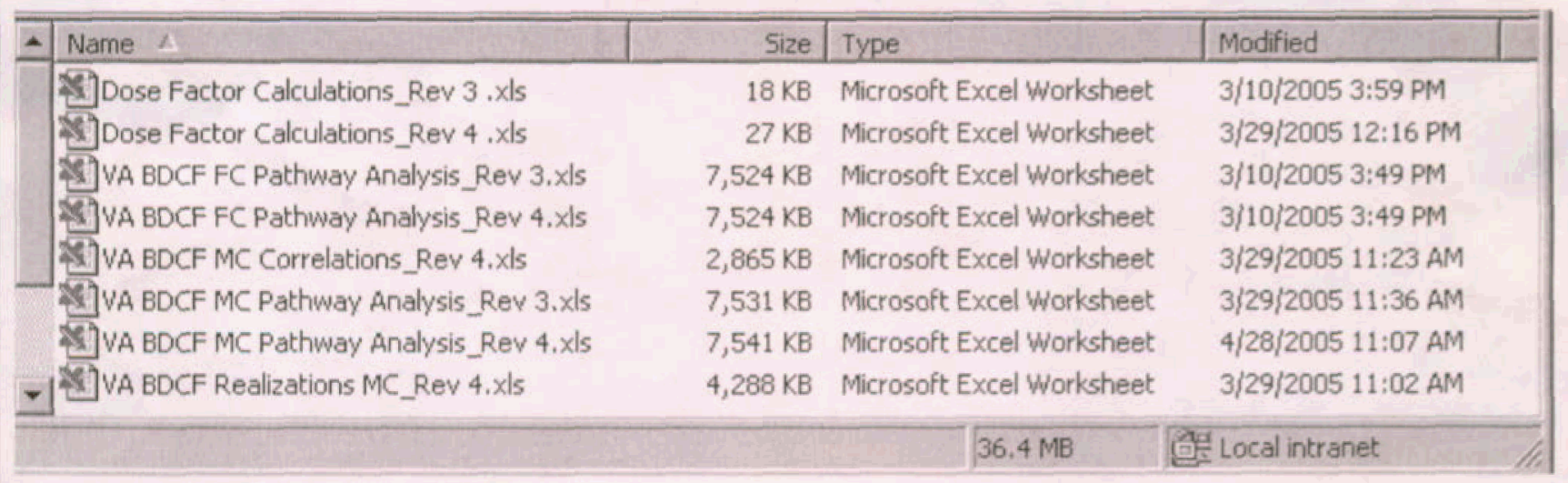

Files transferred to the CD were reviewed and the content and file size checked to validate that the transfer was properly executed.

XREF_ MOL.20050511.0112

$$
\begin{aligned}
& \text { MDS File validation } \\
& \text { TC } 5-15-\varnothing 5
\end{aligned}
$$

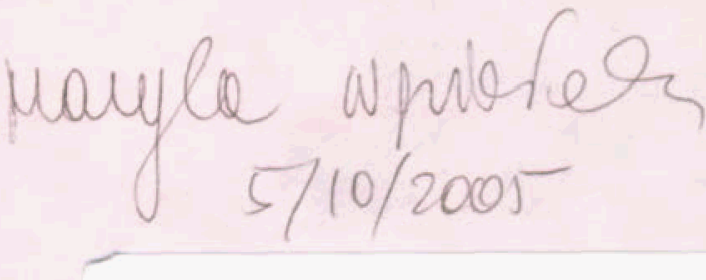

THIS IS AN ELECTRONIC ATTACHMENT 